$$
\begin{aligned}
& \text { AVgebra \& } \\
& \text { d } \\
& \int_{\lrcorner}^{+1} \\
& \text { Number } \\
& \text { Theory }
\end{aligned}
$$

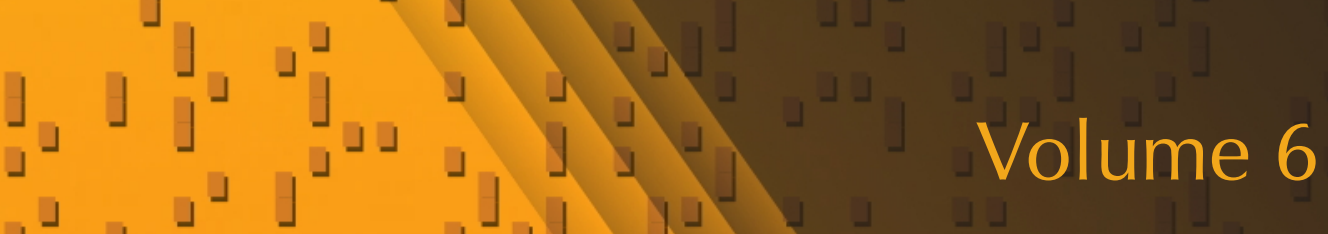

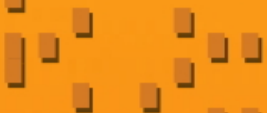

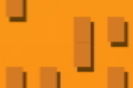

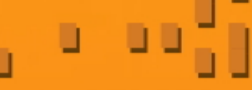

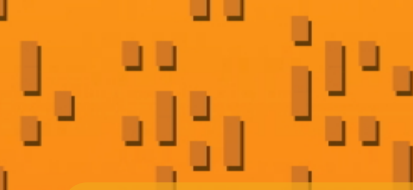

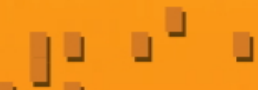

$$
\begin{aligned}
& \lrcorner \\
& \text { No. } 5
\end{aligned}
$$

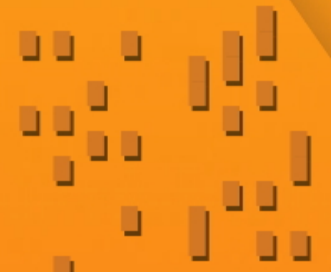

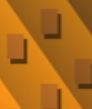

Toroidal compactifications of PEL-type Kuga families

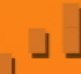

」

.

\lrcorner

$$
\lrcorner\lrcorner\lrcorner \text { Kai-Wen Lan }
$$




\title{
Toroidal compactifications of PEL-type Kuga families
}

\author{
Kai-Wen Lan
}

\begin{abstract}
We explain how compactifications of Kuga families of abelian varieties over PEL-type Shimura varieties, including for example all those products of universal abelian schemes, can be constructed (up to good isogenies not affecting the relative cohomology) by a uniform method. We also calculate the relative cohomology and explain its various properties crucial for applications to the cohomology of automorphic bundles.
\end{abstract}

Introduction

885

1. PEL-type moduli problems and their compactifications 888

2. Kuga families and their compactifications 905

3. Constructions of compactifications and morphisms 915

4. Calculation of formal cohomology 929

5. Polarizations 945

6. Canonical extensions of principal bundles 957

Acknowledgements 963

References $\quad 963$

\section{Introduction}

To study the relations between automorphic forms and Galois representations, it is desirable to understand the cohomology of Shimura varieties with coefficients in algebraic representations of the associated reductive groups (i.e., the so-called automorphic bundles).

In the case of PEL-type Shimura varieties, the associated reductive groups are (up to center) twists of products of symplectic, orthogonal, or general linear groups. According to Weyl's construction [1997] (see also [Fulton and Harris 1991;

This material is based upon works supported by the Clay Liftoff Fellowship, by the National Science Foundation under agreement No. DMS-0635607, and by the Qiu Shi Science and Technology Foundation. Any opinions, findings, and conclusions or recommendations expressed in this material are those of the author and do not necessarily reflect the views of these organizations.

MSC2000: primary 11G18; secondary 11G15, 14D06.

Keywords: Kuga families, PEL-type Shimura varieties, degenerations and toroidal compactifications. 
Goodman and Wallach 2009]), all algebraic representations of a classical group can be realized as summands in the tensor powers of the standard representation of the group. In geometry, one is led to consider the cohomology of fiber products of the universal families of abelian varieties over the PEL-type Shimura varieties. Such fiber products are special cases of what we will call PEL-type Kuga families, or simply Kuga families. When the PEL-type Shimura variety in question is not compact, the total spaces of such Kuga families are not compact either.

To study cohomology properly, one is often led to the question of the existence of projective smooth compactifications with good properties, such as allowing the Hecke operators to act on their cohomology spaces (but not necessarily the geometric spaces). In what follows, let us simply call such compactifications good compactifications. In characteristic zero, such questions can often be handled by the embedded resolution of singularities due to Hironaka [1964a; 1964b]. However, more explicit theories exist in our context. The work of Mumford and his collaborators in [Ash et al. 1975] provides a systematic collection of good compactifications of Shimura varieties with explicit descriptions of local structures, while the work of Pink [1990] provides a systematic construction of good compactifications of the Kuga families as well. These compactifications are called toroidal compactifications. Their methods are analytic in nature and cannot be truly generalized in mixed characteristics.

Based on the theory of degeneration of polarized abelian varieties initiated by Mumford [1972], Faltings and Chai [Faltings 1985; Chai 1985; Faltings and Chai 1990] constructed good compactifications over the integers for Siegel moduli spaces defined by the moduli space of principally polarized abelian varieties. In [Faltings and Chai 1990], they also constructed good compactifications of fiber products of the universal families by gluing weak relatively complete models along the boundary. We ought to point out that, although most works on compactifications spend most of their pages on the construction of boundary charts, it is only the gluing argument that validates the whole construction. (This is not necessarily the case for works using the moduli-theoretic approach, such as [Alexeev and Nakamura 1999; Alexeev 2002; Olsson 2008]. However, the questions there are not less challenging: What can one say about the boundary structures? Are they equally useful for applications to cohomology?) Thus, even if the construction of toroidal compactifications of Siegel moduli spaces in [Faltings and Chai 1990, Chapter IV] has been generalized for all PEL-type Shimura varieties in [Lan 2008], the gluing of weak relatively complete models has to be carried out separately when one works along the original idea of [Faltings and Chai 1990, Chapter VI]. (This is the case in for example [Rozensztajn 2006], in which the assumption that the boundary divisors are regular, i.e., have no crossings, unfortunately rules out all cases where choices of cone decompositions are needed for the Shimura varieties.) 
Note that gluing is not just about techniques of descent. Any theory of descent requires an input of some descent data. Since a naive generalization of the constructions in [Faltings and Chai 1990, Chapter IV] introduces unwanted boundary components, which have to be studied and removed carefully by imposing liftability and pairing conditions as in [Lan 2008], we have reason to believe that a naive generalization of the construction in [Faltings and Chai 1990, Chapter VI, §1] requires delicate modifications, without which even the strongest descent theory cannot be applied.

The aim of this article is to avoid any further argument of gluing, and to treat all PEL-type cases on an equal footing. We shall reduce the construction of toroidal compactifications of PEL-type Kuga families to the construction of toroidal compactifications of Shimura varieties in [Lan 2008], by systematically realizing the Kuga families as locally closed boundary strata in the toroidal compactifications of (larger) PEL-type Shimura varieties. Partly inspired by Kato's theory of log abelian schemes, we can show that, up to refinements of cone decompositions, the structural morphisms from the Kuga families to the Shimura varieties extend (up to good isogenies not affecting the relative cohomology) to log smooth morphisms with nice properties between the toroidal compactifications. This approach differs fundamentally from the one in [Faltings and Chai 1990, Chapter VI]. As Chai pointed out, although no technique can be truly shared between analytic and algebraic constructions, our idea is close in spirit to that of [Pink 1990]. (See Remark 3.10 below.)

Since we replace Faltings and Chai's construction with a different one, we need to explain that our simpler (but perhaps cruder) construction is not less useful. Thus our second task is to calculate the relative (log) de Rham cohomology of the compactified families. We show that such relative cohomology not only enjoys the same expected properties as in [Faltings and Chai 1990, Chapter VI, §1], but also admits natural Hecke actions defined by parabolic subgroups of larger reductive algebraic groups, because our construction uses toroidal boundaries of larger Shimura varieties. This exhibits a large class of endomorphisms on our cohomology spaces, including ones needed in the geometric realization of Weyl's construction (i.e., the realization of automorphic bundles as summands in the relative cohomology of Kuga families).

The outline of this article is as follows. In Section 1, we review some of the results we need from [Lan 2008]. We consider the investment of this summary worthwhile because, although we do not need to carry out another gluing argument, we do need the full strength of the long work [Lan 2008]. In Section 2, we define what we mean by PEL-type Kuga families, state our main theorem, and give an outline of the proof. In Section 3, we carry out the construction of toroidal compactifications for these Kuga families that admit log smooth morphisms to the 
Shimura varieties in question. (This section serves roughly the same purpose as [Faltings and Chai 1990, Chapter VI, §1].) In Sections 4 and 5, we show that these toroidal compactifications are indeed good by justifying what we mentioned in the previous paragraph. (These two sections serve roughly the same purpose as [Faltings and Chai 1990, Chapter VI, §2].) We would like to mention that the use of nerve spectral sequences in Section 4 imitates immediate analogues in [Harris and Zucker 1994; 2001] (based on techniques that can be traced back to [Kempf et al. 1973, Chapter I, §3]), while the use of log extensions of polarizations is inspired by Kato's idea of (relative) log Picard groups [Illusie 1994, 3.3]. (See Remark 5.7.) The article ends with Section 6, in which we explain how to define canonical extensions of the so-called principal bundles.

Although used as the main motivation for our construction, applications to cohomology of automorphic bundles will be deferred to some forthcoming papers. There the readers will find the construction of proper smooth integral models useful for studying cohomology with not only rational coefficients, but also integral and torsion coefficients.

We shall follow [Lan 2008, Notations and Conventions] unless otherwise specified. (Although our references to [Lan 2008] use the numbering in the original version, the reader is advised to consult the errata and revision (available online) for corrections of typos and minor mistakes, and for improved exposition.)

\section{PEL-type moduli problems and their compactifications}

In this section, we summarize definitions and main results in [Lan 2008] that will be needed in this article. We will emphasize definitions such as the ones involved in the description of boundary structures, but will have to be less comprehensive on some fundamental definitions including the ones of level structures.

1A. Linear algebraic data. Let $\mathcal{O}$ be an order in a finite-dimensional semisimple algebra over $\mathbb{Q}$ with a positive involution ${ }^{\star}$. Here an involution means an antiautomorphism of order two, and positivity of ${ }^{\star}$ means $\operatorname{Tr}_{\mathcal{O} \otimes_{\mathbb{Z}} \mathbb{R} / \mathbb{R}}\left(x x^{\star}\right)>0$ for any $x \neq 0$ in $\mathcal{O} \otimes_{\mathbb{Z}} \mathbb{R}$. We assume that $\mathcal{O}$ is mapped to itself under ${ }^{\star}$. We shall denote the center of $\mathcal{O} \otimes_{\mathbb{Z}} \mathbb{Q}$ by $F$.

Let $\mathbb{Z}(1):=\operatorname{ker}\left(\exp : \mathbb{C} \rightarrow \mathbb{C}^{\times}\right)$, which is a free $\mathbb{Z}$-module of rank one. Any choice $\sqrt{-1}$ of a square-root of -1 in $\mathbb{C}$ determines an isomorphism $(2 \pi \sqrt{-1})^{-1}$ : $\mathbb{Z}(1) \stackrel{\sim}{\longrightarrow} \mathbb{Z}$, but there is no canonical isomorphism between $\mathbb{Z}(1)$ and $\mathbb{Z}$. For any commutative $\mathbb{Z}$-algebra $R$, we denote by $R(1)$ the module $R \otimes_{\mathbb{Z}} \mathbb{Z}(1)$.

By a PEL-type $\mathcal{O}$-lattice $(L,\langle\cdot, \cdot\rangle, h)$ (as in [Lan 2008, Definition 1.2.1.3]), we mean the following data:

(1) An $\mathcal{O}$-lattice, namely a $\mathbb{Z}$-lattice $L$ with the structure of an $\mathcal{O}$-module. 
(2) An alternating pairing $\langle\cdot, \cdot\rangle: L \times L \rightarrow \mathbb{Z}(1)$ satisfying $\langle b x, y\rangle=\left\langle x, b^{\star} y\right\rangle$ for any $x, y \in L$ and $b \in \mathcal{O}$, together with an $\mathbb{R}$-algebra homomorphism $h: \mathbb{C} \rightarrow \operatorname{End}_{\mathcal{O} \otimes_{\mathbb{Z}} \mathbb{R}}\left(L \otimes_{\mathbb{Z}} \mathbb{R}\right)$ satisfying:

(a) For any $z \in \mathbb{C}$ and $x, y \in L \otimes_{\mathbb{Z}} \mathbb{R}$, we have $\langle h(z) x, y\rangle=\left\langle x, h\left(z^{c}\right) y\right\rangle$, where $\mathbb{C} \rightarrow \mathbb{C}: z \mapsto z^{c}$ is the complex conjugation.

(b) For any choice of $\sqrt{-1}$ in $\mathbb{C}$, the $\mathbb{R}$-bilinear pairing

$$
(2 \pi \sqrt{-1})^{-1}\langle\cdot h(\sqrt{-1}) \cdot\rangle:\left(L \otimes_{\mathbb{Z}} \mathbb{R}\right) \times\left(L \otimes_{\mathbb{Z}} \mathbb{R}\right) \rightarrow \mathbb{R}
$$

is symmetric and positive definite. (This last condition forces $\langle\cdot, \cdot\rangle$ to be nondegenerate.)

The tuple $\left(\mathcal{O},{ }^{\star}, L,\langle\cdot, \cdot\rangle, h\right)$ (over $\left.\mathbb{Z}\right)$ then gives us an integral version of the tuple $\left(B,{ }^{\star}, V,\langle\cdot, \cdot\rangle, h\right)$ (over $\mathbb{Q}$ ) in [Kottwitz 1992] and related works. (We favor lattices over $\mathbb{Z}$ rather than their analogues over $\mathbb{Q}$ (or over $\mathbb{Z}_{(p)}$ for some $p$ ) because we will work with isomorphism classes rather than isogeny classes; cf. Remark 1.7.)

Definition 1.1 [Lan 2008, Definition 1.2.1.5]. Let a PEL-type $\mathcal{O}$-lattice $(L,\langle\cdot, \cdot\rangle, h)$ be given as above. For any $\mathbb{Z}$-algebra $R$, set

$$
\mathrm{G}(R):=\left\{(g, r) \in \mathrm{GL}_{\mathcal{O} \otimes_{\mathbb{Z}} R}\left(L \otimes_{\mathbb{Z}} R\right) \times \mathbf{G}_{\mathrm{m}}(R):\langle g x, g y\rangle=r\langle x, y\rangle, \forall x, y \in L\right\} .
$$

In other words, $\mathrm{G}(R)$ is the group of symplectic automorphisms of $L \otimes_{\mathbb{Z}} R$ (respecting the pairing $\langle\cdot, \cdot\rangle$ up to a scalar multiple; cf. [Lan 2008, Definition 1.1.4.11]). For any $\mathbb{Z}$-algebra homomorphism $R \rightarrow R^{\prime}$, we have by definition a natural homomorphism $\mathrm{G}(R) \rightarrow \mathrm{G}\left(R^{\prime}\right)$, making $\mathrm{G}$ a group functor (or in fact an affine group scheme) over $\mathbb{Z}$.

The projection to the second factor $(g, r) \mapsto r$ defines a morphism $v: \mathrm{G} \rightarrow \mathbf{G}_{\mathrm{m}}$, which we call the similitude character. For simplicity, we shall often denote elements $(g, r)$ in G by simply $g$, and denote by $v(g)$ the value of $r$ when we need it. (If $L \neq\{0\}$, then the value of $r$ is uniquely determined by $g$. Hence there is little that we lose when suppressing $r$ from the notation. However, this is indeed an abuse of notation when $L=\{0\}$, in which case we have $\mathrm{G}=\mathbf{G}_{\mathrm{m}}$.)

Let $\square$ be any set of rational primes. (It can be either an empty set, a finite set, or an infinite set.) We denote by $\mathbb{Z}_{(\square)}$ the unique localization of $\mathbb{Z}$ (at the multiplicative subset of $\mathbb{Z}$ generated by nonzero integers prime to $\square$ ) having $\square$ as its set of height one primes, and denote by $\hat{\mathbb{Z}}^{\square}$ (resp. $\mathbb{A}^{\infty, \square}$, resp. $\mathbb{A}^{\square}$ ) the integral adeles (resp. finite adeles, resp. adeles) away from $\square$. Then we have definitions for $\mathrm{G}(\mathbb{Q}), \mathrm{G}\left(\mathbb{A}^{\infty}, \square\right), \mathrm{G}\left(\mathbb{A}^{\infty}\right), \mathrm{G}(\mathbb{R}), \mathrm{G}\left(\mathbb{A}^{\square}\right), \mathrm{G}(\mathbb{A}), \mathrm{G}(\mathbb{Z}), \mathrm{G}(\mathbb{Z} / n \mathbb{Z}), \mathrm{G}\left(\hat{\mathbb{Z}}^{\square}\right), \mathrm{G}(\hat{\mathbb{Z}})$, $\mathcal{U}^{\square}(n):=\operatorname{ker}\left(\mathrm{G}\left(\hat{\mathbb{Z}}^{\square}\right) \rightarrow \mathrm{G}\left(\hat{\mathbb{Z}}^{\square} / n \hat{\mathbb{Z}}^{\square}\right)=\mathrm{G}(\mathbb{Z} / n \mathbb{Z})\right)$ for any $n$ prime to $\square$, and $\mathcal{U}(n):=\operatorname{ker}(\mathrm{G}(\hat{\mathbb{Z}}) \rightarrow \mathrm{G}(\hat{\mathbb{Z}} / n \hat{\mathbb{Z}})=\mathrm{G}(\mathbb{Z} / n \mathbb{Z}))$.

Following Pink [1990, 0.6], we define the neatness of open compact subgroups $\mathcal{H}$ of $\mathrm{G}\left(\hat{\mathbb{Z}}^{\square}\right)$ as follows: View $\mathrm{G}\left(\hat{\mathbb{Z}}^{\square}\right)$ as a subgroup of $\mathrm{GL}_{\mathcal{O} \otimes_{\mathbb{Z}} \hat{\mathbb{Z}}}\left(L \otimes_{\mathbb{Z}} \hat{\mathbb{Z}}^{\square}\right) \times \mathbf{G}_{\mathrm{m}}\left(\hat{\mathbb{Z}}^{\square}\right)$. 
(Or we may use any faithful linear algebraic representation of G.) Then, for each rational prime $p>0$ not in $\square$, it makes sense to talk about eigenvalues of elements $g_{p}$ in $\mathrm{G}\left(\mathbb{Z}_{p}\right)$, which are elements in $\overline{\mathbb{Q}}_{p}^{\times}$. Let $g=\left(g_{p}\right) \in \mathrm{G}\left(\hat{\mathbb{Z}}^{\square}\right)$, with $p$ running through rational primes such that $\square \nmid p$. For each such $p$, let $\Gamma_{g_{p}}$ be the subgroup of $\overline{\mathbb{Q}}_{p}^{\times}$generated by eigenvalues of $g_{p}$. For any embedding $\overline{\mathbb{Q}} \hookrightarrow \overline{\mathbb{Q}}_{p}$, consider the subgroup $\left(\overline{\mathbb{Q}}^{\times} \cap \Gamma_{g_{p}}\right.$ ) tors of torsion elements of $\overline{\mathbb{Q}}^{\times} \cap \Gamma_{g_{p}}$, which is independent of the choice of the embedding $\overline{\mathbb{Q}} \hookrightarrow \overline{\mathbb{Q}}_{p}$.

Definition 1.2 [Lan 2008, Definition 1.4.1.8]. We say that $g=\left(g_{p}\right)$ is neat if $\bigcap_{p \notin \square}\left(\overline{\mathbb{Q}}^{\times} \cap \Gamma_{g_{p}}\right)_{\text {tors }}=\{1\}$. We say that an open compact subgroup $\mathcal{H}$ of $G\left(\hat{\mathbb{Z}}^{\square}\right)$ is neat if all its elements are neat.

Remark 1.3. The usual Serre's lemma that no nontrivial root of unity can be congruent to 1 modulo $n$ if $n \geq 3$ shows that $\mathcal{H}$ is neat if $\mathcal{H} \subset \mathcal{U}^{\square}(n)$ for some $n \geq 3$ such that $\square \nmid n$.

Remark 1.4. Definition 1.2 makes no reference to the group $G(\mathbb{Q})$ of rational elements. For the related notion of neatness for arithmetic groups, see [Borel 1969, 17.1].

1B. Definition of moduli problems. Let us fix a PEL-type $\mathcal{O}$-lattice $(L,\langle\cdot, \cdot\rangle, h)$ as in the previous section. Let $F_{0}$ be the reflex field of $\left(L \otimes_{\mathbb{Z}} \mathbb{R},\langle\cdot, \cdot\rangle, h\right)$ defined as in [Kottwitz 1992, page 389] or [Lan 2008, Definition 1.2.5.4]. We shall denote the ring of integers in $F_{0}$ by $\mathcal{O}_{F_{0}}$, and use similar notations for other number fields. (This is in conflict with the notation of the order $\mathcal{O}$, but the precise interpretation will be clear from the context.)

Let Disc $=$ Disc $_{\mathcal{O} / \mathbb{Z}}$ be the discriminant of $\mathcal{O}$ over $\mathbb{Z}$ (as in [Lan 2008, Definition 1.1.1.6]; see also [Lan 2008, Proposition 1.1.1.12]). Closely related to Disc is the invariant $\mathrm{I}_{\mathrm{bad}}$ for $\mathcal{O}$ defined in [Lan 2008, Definition 1.2.1.17], which is either 2 or 1 , depending on whether type $\mathrm{D}$ factors are involved. Let $L^{\#}:=\left\{x \in L \otimes_{\mathbb{Z}} \mathbb{Q}\right.$ : $\langle x, y\rangle \in \mathbb{Z}(1), \forall y \in L\}$ denote the dual lattice of $L$ with respect to the pairing $\langle\cdot, \cdot\rangle$.

Definition 1.5. We say that a prime number $p$ is bad if $p \mid \mathrm{I}_{\mathrm{bad}} \operatorname{Disc}\left[L^{\#}: L\right]$. We say a prime number $p$ is good if it is not bad. We say that $\square$ is a set of good primes if it does not contain any bad primes.

Let us fix a choice of a set $\square$ of good primes. By abuse of notation, let $\mathcal{O}_{F_{0}}$, ( $\square$ ) be the localization of $\mathcal{O}_{F_{0}}$ at the multiplicative set generated by rational prime numbers not in $\square$. Let $S_{0}:=\operatorname{Spec}\left(\mathcal{O}_{F_{0}}\right.$, $\left.\square\right)$ and let $\left(\operatorname{Sch} / S_{0}\right)$ be the category of schemes over $S_{0}$. For any open compact subgroup $\mathcal{H}$ of $\mathrm{G}\left(\hat{\mathbb{Z}}^{\square}\right)$, there is an associated moduli problem $\mathrm{M}_{\mathcal{H}}$ defined as follows:

Definition 1.6 [Lan 2008, Definition 1.4.1.4]. The moduli problem $\mathrm{M}_{\mathcal{H}}$ is defined as the category fibered in groupoids over $\left(\mathrm{Sch} / \mathrm{S}_{0}\right)$ whose fiber over each 
$S$ is the groupoid $\mathrm{M}_{\mathcal{H}}(S)$ described as follows: The objects of $\mathrm{M}_{\mathcal{H}}(S)$ are tuples $\left(G, \lambda, i, \alpha_{\mathcal{H}}\right)$, where:

(1) $G$ is an abelian scheme over $S$.

(2) $\lambda: G \rightarrow G^{\vee}$ is a polarization of degree prime to $\square$.

(3) $i: \mathcal{O} \rightarrow \operatorname{End}_{S}(G)$ defines an $\mathcal{O}$-structure of $(G, \lambda)$ (satisfying the Rosati condition $i(b)^{\vee} \circ \lambda=\lambda \circ i\left(b^{\star}\right)$ for any $\left.b \in \mathcal{O}\right)$.

(4) $\underline{\mathrm{Lie}}_{G / S}$ with its $\mathcal{O} \otimes_{\mathbb{Z}} \mathbb{Z}_{(\square)}$-module structure given naturally by $i$ satisfies the determinantal condition in [Lan 2008, Definition 1.3.4.2] given by

$$
\left(L \otimes_{\mathbb{Z}} \mathbb{R},\langle\cdot, \cdot\rangle, h\right) .
$$

(5) $\alpha_{\mathcal{H}}$ is an (integral) level- $\mathcal{H}$ structure of $(G, \lambda, i)$ of type $\left(L \otimes_{\mathbb{Z}} \hat{\mathbb{Z}}^{\square},\langle\cdot, \cdot\rangle\right)$ as in [Lan 2008, Definition 1.3.7.8].

The isomorphisms $\left(G, \lambda, i, \alpha_{\mathcal{H}}\right) \sim_{\text {isom. }}\left(G^{\prime}, \lambda^{\prime}, i^{\prime}, \alpha_{\mathcal{H}}^{\prime}\right)$ of $\mathrm{M}_{\mathcal{H}}(S)$ are given by (naive) isomorphisms $f: G \stackrel{\sim}{\longrightarrow} G^{\prime}$ such that $\lambda=f^{\vee} \circ \lambda^{\prime} \circ f, f \circ i(b)=i^{\prime}(b) \circ f$ for all $b \in \mathcal{O}$, and $f \circ \alpha_{\mathcal{H}}=\alpha_{\mathcal{H}}^{\prime}$ (symbolically).

Remark 1.7. The definition here using isomorphism classes is not as canonical as the ones proposed by Grothendieck and Deligne using quasiisogeny classes (as in [Kottwitz 1992]). For the relation between their definitions and ours, see [Lan $2008, \S 1.4]$. We introduce the definition (using isomorphisms) here mainly because this is the definition most concrete for the study of compactifications.

Theorem 1.8 [Lan 2008, Theorem 1.4.1.12 and Corollary 7.2.3.10]. The moduli problem $\mathrm{M}_{\mathcal{H}}$ is a smooth separated algebraic stack of finite type over $\mathrm{S}_{0}$. It is representable by a quasiprojective scheme if the objects it parametrizes have no nontrivial automorphism, which is in particular the case when $\mathcal{H}$ is neat (as in Definition 1.2).

We shall insist from now on the following technical condition on PEL-type O-lattices:

Condition 1.9 [Lan 2008, Condition 1.4.3.9]. The PEL-type $\mathcal{O}$-lattice $(L,\langle\cdot, \cdot\rangle, h)$ is chosen such that the action of $\mathcal{O}$ on $L$ extends to an action of some maximal order $\mathcal{O}^{\prime}$ in $B$ containing $\mathcal{O}$.

1C. Cusp labels. Although there is no rational boundary components in the theory of arithmetic compactifications (in mixed characteristics), we have developed in [Lan 2008, §5.4] the notion of cusp labels that serves a similar purpose. (While $\mathrm{G}(\mathbb{Q})$ plays an important role in the analytic theory over $\mathbb{C}$, it does not play any obvious role in the algebraic theory over $\mathcal{O}_{F_{0}}$, $\square$. This is partly due to the so-called failure of Hasse's principle; see for example [Kottwitz 1992, §8] and [Lan 2008, Remark 1.4.3.11].) 
Unlike in the analytic theory over $\mathbb{C}$, where boundary components are naturally parametrized by group-theoretic objects, the only algebraic machinery we have is the theory of semiabelian degenerations of abelian varieties with PEL structures. The cusp labels are (by their very design) part of the parameters (which we call the degeneration data) for such (semiabelian) degenerations.

Definition 1.10 [Lan 2008, §1.2.6]. Let $R$ be any noetherian $\mathbb{Z}$-algebra. Suppose we have an increasing filtration $\mathrm{F}=\left\{\mathrm{F}_{-i}\right\}$ on $L \otimes_{\mathbb{Z}} R$, indexed by nonpositive integers $-i$, such that $\mathrm{F}_{0}=L \otimes_{\mathbb{Z}} R$.

(1) We say that $\mathrm{F}$ is integrable if, for any $i, \mathrm{Gr}_{-i}^{\mathrm{F}}:=\mathrm{F}_{-i} / \mathrm{F}_{-i-1}$ is integrable in the sense that $\mathrm{Gr}_{-i}^{\mathrm{F}} \cong M_{i} \otimes_{\mathbb{Z}} R$ (as $R$-modules) for some $\mathcal{O}$-lattice $M_{i}$.

(2) We say that $\mathrm{F}$ is split if there exists (noncanonically) some isomorphism $\mathrm{Gr}^{\mathrm{F}}:=\oplus_{i} \mathrm{Gr}_{-i}^{\mathrm{F}} \stackrel{\sim}{\longrightarrow} \mathrm{F}_{0}$ of $R$-modules.

(3) We say that $\mathrm{F}$ is admissible if it is both integrable and split.

(4) Let $m$ be an integer. We say that $\mathrm{F}$ is $m$-symplectic with respect to $(L,\langle\cdot, \cdot\rangle)$ if, for any $i, \mathrm{~F}_{-m+i}$ and $\mathrm{F}_{-i}$ are annihilators of each other under the pairing $\langle\cdot, \cdot\rangle$ on $\mathrm{F}_{0}$.

We shall only work with $m=3$, and we shall suppress $m$ in what follows. The fact that $\hat{\mathbb{Z}}^{\square}$ involves bad primes (cf. Definition 1.5) is the main reason that we may have to allow nonprojective filtrations.

Definition 1.11 [Lan 2008, Definition 5.2.7.1]. We say that a symplectic admissible filtration $\mathrm{Z}$ on $L \otimes_{\mathbb{Z}} \hat{\mathbb{Z}}^{\square}$ is fully symplectic with respect to $(L,\langle\cdot, \cdot\rangle)$ if there is a symplectic admissible filtration $\mathrm{Z}_{\mathbb{A}} \square=\left\{Z_{-i, \mathbb{A}} \square\right\}$ on $L \otimes_{\mathbb{Z}} \mathbb{A}^{\square}$ that extends $\mathrm{Z}$ in the sense that $\mathrm{Z}_{-i, \mathbb{A}^{\square}} \cap\left(L \otimes_{\mathbb{Z}} \hat{\mathbb{Z}}^{\square}\right)=\mathrm{Z}_{-i}$ in $L \otimes_{\mathbb{Z}} \mathbb{A}^{\square}$ for all $i$.

Definition 1.12 [Lan 2008, Definition 5.2.7.3]. A symplectic-liftable admissible filtration $Z_{n}$ on $L / n L$ is called fully symplectic-liftable with respect to $(L,\langle\cdot, \cdot\rangle)$ if it is the reduction modulo $n$ of some admissible filtration $\mathrm{Z}$ on $L \otimes_{\mathbb{Z}} \hat{\mathbb{Z}}^{\square}$ that is fully symplectic with respect to $(L,\langle\cdot, \cdot\rangle)$ as in Definition 1.11 .

Degenerations into semiabelian schemes induce filtrations on Tate modules and on Lie algebras of the generic fibers. While the symplectic-liftable admissible filtrations represent (certain orbits of) filtrations on $L \otimes_{\mathbb{Z}} \hat{\mathbb{Z}}^{\square}$ induced by filtrations on Tate modules via the level structures, the fully symplectic-liftable ones are equipped with (certain orbits of) filtrations on $L \otimes_{\mathbb{Z}} \mathbb{R}$ induced by the filtrations on Lie algebras via the Lie algebra condition (4) in Definition 1.6. (One may interpret the Lie algebra condition as the "de Rham" (or rather "Hodge") component of a certain "complete level structure", the direct product of whose " $\ell$-adic" components being a level structure in the usual sense.) Such (orbits of) filtrations are the crudest invariants of degenerations we consider. 
Definition 1.13 [Lan 2008, Definition 5.4.1.3]. Given a fully symplectic admissible filtration $\mathrm{Z}$ on $L \otimes_{\mathbb{Z}} \hat{\mathbb{Z}}^{\square}$ with respect to $(L,\langle\cdot, \cdot\rangle)$ as in Definition 1.11, a torus argument $\Phi$ for $\mathrm{Z}$ is a tuple $\Phi:=\left(X, Y, \phi, \varphi_{-2}, \varphi_{0}\right)$, where:

(1) $X$ and $Y$ are $\mathcal{O}$-lattices of the same $\mathcal{O}$-multirank (see [Lan 2008, Definition 5.2.2.5]), and $\phi: Y \hookrightarrow X$ is an $\mathcal{O}$-equivariant embedding.

(2) $\varphi_{-2}: \mathrm{Gr}_{-2}^{\mathrm{Z}} \stackrel{\sim}{\longrightarrow} \operatorname{Hom}_{\hat{\mathbb{Z}} \square}\left(X \otimes_{\mathbb{Z}} \hat{\mathbb{Z}}^{\square}, \hat{\mathbb{Z}}^{\square}(1)\right)$ and $\varphi_{0}: \mathrm{Gr}_{0}^{\mathrm{Z}} \stackrel{\sim}{\rightarrow} Y \otimes_{\mathbb{Z}} \hat{\mathbb{Z}}^{\square}$ are isomorphisms (of $\hat{\mathbb{Z}}^{\square}$-modules) such that the pairing $\langle\cdot, \cdot\rangle_{20}: \mathrm{Gr}_{-2}^{\mathrm{Z}} \times \mathrm{Gr}_{0}^{Z} \rightarrow$ $\hat{\mathbb{Z}}^{\square}$ (1) defined by $\mathrm{Z}$ is the pullback of the pairing

$$
\langle\cdot, \cdot\rangle_{\phi}: \operatorname{Hom}_{\hat{\mathbb{Z}} \square}\left(X \otimes_{\mathbb{Z}} \hat{\mathbb{Z}}^{\square}, \hat{\mathbb{Z}}^{\square}(1)\right) \times\left(Y \otimes_{\mathbb{Z}} \hat{\mathbb{Z}}^{\square}\right) \rightarrow \hat{\mathbb{Z}}^{\square}(1)
$$

defined by the composition

$$
\begin{aligned}
\operatorname{Hom}_{\hat{\mathbb{Z}} \square}\left(X \otimes_{\mathbb{Z}} \hat{\mathbb{Z}}^{\square}, \hat{\mathbb{Z}}^{\square}(1)\right) \times\left(Y \otimes_{\mathbb{Z}} \hat{\mathbb{Z}}^{\square}\right) & \\
& \stackrel{\operatorname{Id} \times \phi}{\rightarrow} \operatorname{Hom}_{\hat{\mathbb{Z}} \square}\left(X \otimes_{\mathbb{Z}} \hat{\mathbb{Z}}^{\square}, \hat{\mathbb{Z}}^{\square}(1)\right) \times\left(X \otimes_{\mathbb{Z}} \hat{\mathbb{Z}}^{\square}\right) \rightarrow \hat{\mathbb{Z}}^{\square}(1),
\end{aligned}
$$

with the sign convention that $\langle\cdot, \cdot\rangle_{\phi}(x, y)=x(\phi(y))=(\phi(y))(x)$ for any $x \in \operatorname{Hom}_{\hat{\mathbb{Z}} \square}\left(X \otimes_{\mathbb{Z}} \hat{\mathbb{Z}}^{\square}, \hat{\mathbb{Z}}^{\square}(1)\right)$ and any $y \in Y \otimes_{\mathbb{Z}} \hat{\mathbb{Z}}^{\square}$.

Definition 1.14 [Lan 2008, Definitions 5.4.1.4 and 5.4.1.5]. Given a fully symplecticliftable admissible filtration $\mathrm{Z}_{n}$ on $L / n L$ with respect to $(L,\langle\cdot, \cdot\rangle)$ as in Definition 1.12 , a torus argument $\Phi_{n}$ at level $n$ for $\mathrm{Z}_{n}$ is a tuple $\Phi_{n}:=\left(X, Y, \phi, \varphi_{-2, n}, \varphi_{0, n}\right)$, where:

(1) $X$ and $Y$ are $\mathcal{O}$-lattices of the same $\mathcal{O}$-multirank, and $\phi: Y \hookrightarrow X$ is an $\mathcal{O}$-equivariant embedding.

(2) $\varphi_{-2, n}: \mathrm{Gr}_{-2, n}^{Z} \stackrel{\sim}{\longrightarrow} \operatorname{Hom}(X / n X,(\mathbb{Z} / n \mathbb{Z})(1))\left(\right.$ resp. $\left.\varphi_{0, n}: \mathrm{Gr}_{0, n}^{Z} \stackrel{\sim}{\longrightarrow} Y / n Y\right)$ is an isomorphism that is the reduction modulo $n$ of some isomorphism $\varphi_{-2}$ : $\mathrm{Gr}_{-2}^{\mathrm{Z}} \stackrel{\sim}{\longrightarrow} \operatorname{Hom}_{\hat{\mathbb{Z}} \square}\left(X \otimes_{\mathbb{Z}} \hat{\mathbb{Z}}^{\square}, \hat{\mathbb{Z}}^{\square}(1)\right)\left(\right.$ resp. $\left.\varphi_{0}: \mathrm{Gr}_{0}^{Z} \stackrel{\sim}{\longrightarrow}\left(Y \otimes_{\mathbb{Z}} \hat{\mathbb{Z}}^{\square}\right)\right)$, such that $\Phi=\left(X, Y, \phi, \varphi_{-2}, \varphi_{0}\right)$ form a torus argument as in Definition 1.13.

We say in this case that $\Phi_{n}$ is the reduction modulo $n$ of $\Phi$.

Two torus arguments $\Phi_{n}=\left(X, Y, \phi, \varphi_{-2, n}, \varphi_{0, n}\right)$ and $\Phi_{n}^{\prime}=\left(X^{\prime}, Y^{\prime}, \phi^{\prime}, \varphi_{-2, n}^{\prime}, \varphi_{0, n}^{\prime}\right)$ at level $n$ are equivalent if and only if there exists a pair of isomorphisms

$$
\left(\gamma_{X}: X^{\prime} \stackrel{\sim}{\longrightarrow} X, \gamma_{Y}: Y \stackrel{\sim}{\longrightarrow} Y^{\prime}\right)
$$

(of $\mathcal{O}$-lattices) such that $\phi=\gamma_{X} \phi^{\prime} \gamma_{Y}, \varphi_{-2, n}^{\prime}={ }^{t} \gamma_{X} \varphi_{-2, n}$, and $\varphi_{0, n}^{\prime}=\gamma_{Y} \varphi_{0, n}$. In this case, we say that $\Phi_{n}$ and $\Phi_{n}^{\prime}$ are equivalent under the pair of isomorphisms $\gamma=\left(\gamma_{X}, \gamma_{Y}\right)$, which we denote as $\gamma=\left(\gamma_{X}, \gamma_{Y}\right): \Phi_{n} \stackrel{\sim}{\rightarrow} \Phi_{n}^{\prime}$.

The torus arguments record the isomorphism classes of the torus parts of degenerations of abelian schemes with PEL structures. These are the second crudest invariants of degenerations we consider. 
Definition 1.15 [Lan 2008, Definition 5.4.1.9]. A (principal) cusp label at level $n$ for a PEL-type $\mathcal{O}$-lattice $(L,\langle\cdot, \cdot\rangle, h)$, or a cusp label of the moduli problem $\mathrm{M}_{n}$, is an equivalence class $\left[\left(\mathrm{Z}_{n}, \Phi_{n}, \delta_{n}\right)\right]$ of triples $\left(\mathrm{Z}_{n}, \Phi_{n}, \delta_{n}\right)$, where:

(1) $Z_{n}$ is an admissible filtration on $L / n L$ that is fully symplectic-liftable in the sense of Definition 1.12.

(2) $\Phi_{n}$ is a torus argument at level $n$ for $Z_{n}$.

(3) $\delta_{n}: \mathrm{Gr}_{n}^{\mathrm{Z}} \stackrel{\sim}{\longrightarrow} L / n L$ is a liftable splitting.

Two triples $\left(\mathrm{Z}_{n}, \Phi_{n}, \delta_{n}\right)$ and $\left(\mathrm{Z}_{n}^{\prime}, \Phi_{n}^{\prime}, \delta_{n}^{\prime}\right)$ are equivalent if $\mathrm{Z}_{n}$ and $\mathrm{Z}_{n}^{\prime}$ are identical, and if $\Phi_{n}$ and $\Phi_{n}^{\prime}$ are equivalent as in Definition 1.14.

The liftable splitting $\delta_{n}$ in any triple $\left(\mathrm{Z}_{n}, \Phi_{n}, \delta_{n}\right)$ is noncanonical and auxiliary in nature. Such splittings are needed for analyzing the "degeneration of pairings" in general PEL cases (unlike in the special case in [Faltings and Chai 1990, Chapter IV, $\S 6])$.

To proceed from principal cusp labels at level $n$ to general cusp labels at level $\mathcal{H}$, where $\mathcal{H}$ is an open compact subgroup of $\mathrm{G}\left(\hat{\mathbb{Z}}^{\square}\right)$, we form étale orbits of the objects we have thus defined. The precise definitions are complicated (see [Lan 2008, Definitions 5.4.2.1, 5.4.2.2, and 5.4.2.4]) but the idea is simple: For any $\mathcal{H}$ as above, consider those $n \geq 1$ sufficiently divisible such that $\square \nmid n$ and $\mathcal{U}^{\square}(n) \subset \mathcal{H}$. Then we have a compatible system of finite groups $\mathcal{H}_{n}=\mathcal{H} / \mathcal{U}^{\square}(n)$, and an object at level $\mathcal{H}$ is simply defined to be a compatible system of étale $\mathcal{H}_{n}$-orbits of objects at running levels $n$ as above. Then we arrive at the notions of torus arguments $\Phi_{\mathcal{H}}=\left(X, Y, \phi, \varphi_{-2, n}, \varphi_{0, n}\right)$ at level $\mathcal{H}$, and of representatives $\left(\mathrm{Z}_{\mathcal{H}}, \Phi_{\mathcal{H}}, \delta_{\mathcal{H}}\right)$ of cusp labels $\left[\left(\mathrm{Z}_{\mathcal{H}}, \Phi_{\mathcal{H}}, \delta_{\mathcal{H}}\right)\right]$ at level $\mathcal{H}$. (The liftability condition is implicit in such a definition, as in the definition of level structures we omitted.) By abuse of language, we call these $\mathcal{H}$-orbits of $\Phi=\left(X, Y, \phi, \varphi_{-2}, \varphi_{0}\right),(\mathrm{Z}, \Phi, \delta)$, and $[(\mathrm{Z}, \Phi, \delta)]$, respectively.

For simplicity, we shall often omit $\mathrm{Z}_{\mathcal{H}}$ from the notation.

Lemma 1.16 [Lan 2008, Lemma 5.2.7.5 in the revision]. Let $\mathrm{Z}_{n}$ be an admissible filtration on $L / n L$ that is fully symplectic-liftable with respect to $(L,\langle\cdot, \cdot\rangle)$. Let $\left(\mathrm{Gr}_{-1}^{\mathrm{Z}},\langle\cdot, \cdot\rangle_{11}\right)$ be induced by some fully symplectic lifting $\mathrm{Z}$ of $\mathrm{Z}_{n}$, and let $\left(\mathrm{Gr}_{-1, \mathbb{R}}^{\mathrm{Z}},\langle\cdot, \cdot\rangle_{11, \mathbb{R}}, h_{-1}\right)$ be determined by [Lan 2008, Proposition 5.1.2.2 in the revision] by any extension $\mathrm{Z}_{\mathcal{A}} \square$ in Definition 1.11 (which has the same reflex field $F_{0}$ as $\left(L \otimes_{\mathbb{Z}} \mathbb{R},\langle\cdot, \cdot\rangle, h\right)$ does). Then there is associated (noncanonically) a PEL-type $\mathcal{O}$-lattice $\left(L_{n}^{\mathrm{Z}},\langle\cdot, \cdot\rangle_{n}^{\mathrm{Z}}, h_{n}^{\mathrm{Z}}\right)$ satisfying Condition 1.9 such that:

(1) $\left[\left(L_{n}^{\mathrm{Z}}\right)^{\#}: L_{n}^{\mathrm{Z}}\right]$ is prime to $\square$.

(2) There exist (noncanonical) $\mathcal{O}$-equivariant isomorphisms

$$
\left(\mathrm{Gr}_{-1}^{\mathrm{Z}},\langle\cdot, \cdot\rangle_{11}\right) \stackrel{\sim}{\longrightarrow}\left(L^{\mathrm{Z}_{n}} \otimes_{\mathbb{Z}} \hat{\mathbb{Z}}^{\square},\langle\cdot, \cdot\rangle^{\mathrm{Z}_{n}}\right)
$$


and

$$
\left(\mathrm{Gr}_{-1, \mathbb{R}}^{\mathrm{Z}},\langle\cdot, \cdot\rangle_{11, \mathbb{R}}, h_{-1}\right) \stackrel{\sim}{\rightarrow}\left(L^{\mathrm{Z}_{n}} \otimes_{\mathbb{Z}} \mathbb{R},\langle\cdot, \cdot\rangle^{\mathrm{Z}_{n}}, h^{\mathrm{Z}_{n}}\right) .
$$

(3) The moduli problem $\mathrm{M}_{n}^{\mathrm{Z}_{n}}$ defined by the noncanonical $\left(L^{\mathrm{Z}_{n}},\langle\cdot, \cdot\rangle^{\mathrm{Z}_{n}}, h^{\mathrm{Z}_{n}}\right)$ as in Definition 1.6 is canonical in the sense that it depends (up to isomorphism) only on $\mathrm{Z}_{n}$, but not on the choice of $\left(L^{\mathrm{Z}_{n}},\langle\cdot, \cdot\rangle^{\mathrm{Z}_{n}}, h^{\mathrm{Z}_{n}}\right)$.

Definition 1.17 [Lan 2008, Definition 5.4.2.6]. The PEL-type $\mathcal{O}$-lattice

$$
\left(L^{\mathrm{Z}_{\mathcal{H}}},\langle\cdot, \cdot\rangle^{\mathrm{Z}_{\mathcal{H}}}, h^{\mathrm{Z}_{\mathcal{H}}}\right)
$$

is a fixed (noncanonical) choice of any of the PEL-type $\mathcal{O}$-lattice $\left(L^{Z_{n}},\langle\cdot, \cdot\rangle^{Z_{n}}, h^{Z_{n}}\right)$ in Lemma 1.16 for any element $Z_{n}$ in any $Z_{\mathcal{H}_{n}}\left(\right.$ in $Z_{\mathcal{H}}=\left\{Z_{\mathcal{H}_{n}}\right\}$, a compatible collection of étale orbits $Z_{\mathcal{H}_{n}}$ at various levels $n$ such that $\square \nmid n$ and $\left.\mathcal{U}^{\square}(n) \subset \mathcal{H}\right)$. The elements

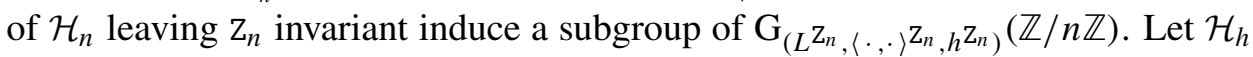
be the preimage of this subgroup under

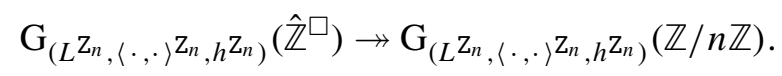

Then we define $\mathrm{M}_{\mathcal{H}}^{Z_{\mathcal{H}}}$ to be the moduli problem defined by $\left(L^{Z_{n}},\langle\cdot, \cdot\rangle^{Z_{n}}, h^{Z_{n}}\right)$ with level- $\mathcal{H}_{h}$ structures as in Lemma 1.16. (The isomorphism class of this final moduli problem is independent of the choice of $\left(L^{\mathrm{Z}_{\mathcal{H}}},\langle\cdot, \cdot\rangle^{\mathrm{Z}_{\mathcal{H}}}, h^{\mathrm{Z}_{\mathcal{H}}}\right)=\left(L^{\mathrm{Z}_{n}},\langle\cdot, \cdot\rangle^{\mathrm{Z}_{n}}, h^{\mathrm{Z}_{n}}\right)$.)

Such boundary moduli problems $\mathrm{M}_{\mathcal{H}}^{Z_{\mathcal{H}}}$ are the fundamental building blocks in the construction of toroidal boundary charts for $\mathrm{M}_{\mathcal{H}}$. (They actually appear in the boundary of the minimal compactification of $\mathrm{M}_{\mathcal{H}}$, which we call cusps. They are parametrized by the cusp labels of $\mathrm{M}_{\mathcal{H}}$.)

It is important to study the relations among cusp labels of different multiranks.

Definition 1.18 [Lan 2008, Definition 5.4.1.15]. A surjection

$$
\left(\mathrm{Z}_{n}, \Phi_{n}, \delta_{n}\right) \rightarrow\left(\mathrm{Z}_{n}^{\prime}, \Phi_{n}^{\prime}, \delta_{n}^{\prime}\right)
$$

between representatives of cusp labels at level $n$, where $\Phi_{n}=\left(X, Y, \phi, \varphi_{-2, n}, \varphi_{0, n}\right)$ and $\Phi_{n}^{\prime}=\left(X^{\prime}, Y^{\prime}, \phi^{\prime}, \varphi_{-2, n}^{\prime}, \varphi_{0, n}^{\prime}\right)$, is a pair (of surjections) $\left(s_{X}: X \rightarrow X^{\prime}, s_{Y}: Y \rightarrow Y^{\prime}\right)$ (of $\mathcal{O}$-lattices) such that:

(1) Both $s_{X}$ and $s_{Y}$ are admissible surjections (i.e., with kernels defining filtrations that are admissible in the sense of Definition 1.10), and they are compatible with $\phi$ and $\phi^{\prime}$ in the sense that $s_{X} \phi=\phi^{\prime} s_{Y}$.

(2) $\mathrm{Z}_{-2, n}^{\prime}$ is an admissible submodule of $\mathrm{Z}_{-2, n}$, and the natural embedding $\mathrm{Gr}_{-2, n}^{\mathrm{Z}^{\prime}} \hookrightarrow$ $\mathrm{Gr}^{\mathrm{Z}_{-2, n}}$ satisfies $\varphi_{-2, n} \circ\left(\mathrm{Gr}_{-2, n}^{\mathrm{Z}^{\prime}} \hookrightarrow \mathrm{Gr}^{\mathrm{Z}_{-2, n}}\right)=s_{X}^{*} \circ \varphi_{-2, n}^{\prime}$.

(3) $\mathrm{Z}_{-1, n}$ is an admissible submodule of $\mathrm{Z}_{-1, n}^{\prime}$, and the natural surjection $\mathrm{Gr}^{\mathrm{Z}_{0, n}} \rightarrow$ $\mathrm{Gr}_{0, n}^{\mathrm{Z}^{\prime}}$ satisfies $s_{Y} \circ \varphi_{0, n}=\varphi_{0, n}^{\prime} \circ\left(\mathrm{Gr}^{\mathrm{Z}_{0, n}} \rightarrow \mathrm{Gr}_{0, n}^{\mathrm{Z}^{\prime}}\right)$. 
In this case, we write $s=\left(s_{X}, s_{Y}\right):\left(\mathrm{Z}_{n}, \Phi_{n}, \delta_{n}\right) \rightarrow\left(\mathrm{Z}_{n}^{\prime}, \Phi_{n}^{\prime}, \delta_{n}^{\prime}\right)$

By taking orbits as before, there is a corresponding notion for general cusp labels: Definition 1.19 [Lan 2008, Definition 5.4.2.12]. A surjection $\left(\mathrm{Z}_{\mathcal{H}}, \Phi_{\mathcal{H}}, \delta_{\mathcal{H}}\right) \rightarrow$ $\left(\mathrm{Z}_{\mathcal{H}}^{\prime}, \Phi_{\mathcal{H}}^{\prime}, \delta_{\mathcal{H}}^{\prime}\right)$ between representatives of cusp labels at level $\mathcal{H}$, where $\Phi_{\mathcal{H}}=$ $\left(X, Y, \phi, \varphi_{-2, \mathcal{H}}, \varphi_{0, \mathcal{H}}\right)$ and $\Phi_{\mathcal{H}}^{\prime}=\left(X^{\prime}, Y^{\prime}, \phi^{\prime}, \varphi_{-2, \mathcal{H}}^{\prime}, \varphi_{0, \mathcal{H}}^{\prime}\right)$, is a pair (of surjections) $s=\left(s_{X}: X \rightarrow X^{\prime}, s_{Y}: Y \rightarrow Y^{\prime}\right)$ (of $\mathcal{O}$-lattices) such that:

(1) Both $s_{X}$ and $s_{Y}$ are admissible surjections, and they are compatible with $\phi$ and $\phi^{\prime}$ in the sense that $s_{X} \phi=\phi^{\prime} s_{Y}$.

(2) $\mathrm{Z}_{\mathcal{H}}^{\prime}$ and $\left(\varphi_{-2, \mathcal{H}}^{\prime}, \varphi_{0, \mathcal{H}}^{\prime}\right)$ are assigned to $\mathrm{Z}_{\mathcal{H}}$ and $\left(\varphi_{-2, \mathcal{H}}, \varphi_{0, \mathcal{H}}\right)$ respectively under $s=\left(s_{X}, s_{Y}\right)$ as in [Lan 2008, Lemma 5.4.2.11].

In this case, we write $s=\left(s_{X}, s_{Y}\right):\left(\mathrm{Z}_{\mathcal{H}}, \Phi_{\mathcal{H}}, \delta_{\mathcal{H}}\right) \rightarrow\left(\mathrm{Z}_{\mathcal{H}}^{\prime}, \Phi_{\mathcal{H}}^{\prime}, \delta_{\mathcal{H}}^{\prime}\right)$.

Definition 1.20 [Lan 2008, Definition 5.4.2.13]. We say that there is a surjection from a cusp label at level $\mathcal{H}$ represented by some $\left(\mathrm{Z}_{\mathcal{H}}, \Phi_{\mathcal{H}}, \delta_{\mathcal{H}}\right)$ to a cusp label at level $\mathcal{H}$ represented by some $\left(\mathrm{Z}_{\mathcal{H}}^{\prime}, \Phi_{\mathcal{H}}^{\prime}, \delta_{\mathcal{H}}^{\prime}\right)$ if there is a surjection $\left(s_{X}, s_{Y}\right)$ from $\left(\mathrm{Z}_{\mathcal{H}}, \Phi_{\mathcal{H}}, \delta_{\mathcal{H}}\right)$ to $\left(\mathrm{Z}_{\mathcal{H}}^{\prime}, \Phi_{\mathcal{H}}^{\prime}, \delta_{\mathcal{H}}^{\prime}\right)$.

This is well defined by [Lan 2008, Lemma 5.4.1.16].

The surjection among cusp labels can be naturally seen when we have the so-called two-step degenerations (see [Faltings and Chai 1990, Chapter III, §10] and [Lan 2008, §4.5.6 in the revision]). This notion will be further developed in Definitions $1.32,1.37$, and 1.38 below.

1D. Cone decompositions. For any torus argument $\Phi_{n}=\left(X, Y, \phi, \varphi_{-2, n}, \varphi_{0, n}\right)$ at level $n$, consider the finitely generated commutative group (i.e., $\mathbb{Z}$-module)

$$
\dddot{\mathbf{S}}_{\Phi_{n}}:=\left(\left(\frac{1}{n} Y\right) \otimes_{\mathbb{Z}} X\right) /\left(\begin{array}{c}
y \otimes \phi\left(y^{\prime}\right)-y^{\prime} \otimes \phi(y) \\
\left(b \frac{1}{n} y\right) \otimes \chi-\left(\frac{1}{n} y\right) \otimes\left(b^{\star} \chi\right)
\end{array}\right)_{\substack{y, y^{\prime} \in Y \\
\chi \in X, b \in \mathcal{O}}}
$$

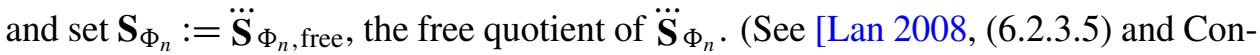
vention 6.2.3.26].) Then, for a general torus argument $\Phi_{\mathcal{H}}=\left(X, Y, \phi, \varphi_{-2, \mathcal{H}}, \varphi_{0, \mathcal{H}}\right)$ at level $\mathcal{H}$, there is a recipe [Lan 2008, Lemma 6.2.4.4] that gives a corresponding free commutative group $\mathbf{S}_{\Phi_{\mathcal{H}}}$ (which can be identified with a finite index subgroup of some $\mathbf{S}_{\Phi_{n}}$ ).

The group $\mathbf{S}_{\Phi_{\mathcal{H}}}$ provides indices for certain "Laurent series expansions" near the boundary strata. In the modular curve case, it is canonically isomorphic to $\mathbb{Z}$, which means there is a canonical parameter $q$ near the boundary - i.e., the cusps. The expansion of modular forms with respect to this parameter then gives the familiar $q$-expansion along the cusps. The compactification of the modular curves can be described locally near each of the cusps by $\operatorname{Spec}\left(R\left[q^{i}\right]_{i \in \mathbb{Z}}\right) \hookrightarrow \operatorname{Spec}\left(R\left[q^{i}\right]_{i \in \mathbb{Z}_{\geq 0}}\right)$ 
for some suitable base ring $R$. For $\mathrm{M}_{\mathcal{H}}$, we would like to have an analogous theory in which the torus with the character group $\mathbf{S}_{\Phi_{\mathcal{H}}}$ can be partially compactified by adding normal crossings divisors in a smooth scheme. This is best achieved by the theory of toroidal embeddings developed in [Kempf et al. 1973]. Many terminologies in such a theory will naturally show up in our description of the toroidal boundary charts, and we will review them in what follows.

Let $\mathbf{S}_{\Phi_{\mathcal{H}}}^{\vee}:=\operatorname{Hom}_{\mathbb{Z}}\left(\mathbf{S}_{\Phi_{\mathcal{H}}}, \mathbb{Z}\right)$ be the $\mathbb{Z}$-dual of $\mathbf{S}_{\Phi_{\mathcal{H}}}$, and let $\left(\mathbf{S}_{\Phi_{\mathcal{H}}}\right)_{\mathbb{R}}^{\vee}:=\mathbf{S}_{\Phi_{\mathcal{H}}}^{\vee} \otimes_{\mathbb{Z}} \mathbb{R}=$ $\operatorname{Hom}_{\mathbb{Z}}\left(\mathbf{S}_{\Phi_{\mathcal{H}}}, \mathbb{R}\right)$. By construction of $\mathbf{S}_{\Phi_{\mathcal{H}}}$, the $\mathbb{R}$-vector space $\left(\mathbf{S}_{\Phi_{\mathcal{H}}}\right)_{\mathbb{R}}^{\vee}$ is isomorphic to the space of Hermitian pairings $(\cdot, \cdot D):\left(Y \otimes_{\mathbb{Z}} \mathbb{R}\right) \times\left(Y \otimes_{\mathbb{Z}} \mathbb{R}\right) \rightarrow \mathcal{O} \otimes_{\mathbb{Z}} \mathbb{R}=B \otimes_{\mathbb{Q}} \mathbb{R}$, by sending a Hermitian pairing $l \cdot, \cdot D$ to the function $y \otimes \phi\left(y^{\prime}\right) \mapsto \operatorname{Tr}_{B / \mathbb{Q}}\left|y, y^{\prime}\right|$ in $\operatorname{Hom}_{\mathbb{R}}\left(\left(Y \otimes_{\mathbb{Z}} \mathbb{R}\right) \times\left(Y \otimes_{\mathbb{Z}} \mathbb{R}\right), \mathbb{R}\right) \cong\left(\mathbf{S}_{\Phi_{\mathcal{H}}}\right)_{\mathbb{R}}^{\vee}$. (See [Lan 2008, Lemma 1.1.4.6].)

Definition 1.22 [Lan 2008, beginning of §6.1.1]. (1) A subset of $\left(\mathbf{S}_{\Phi_{\mathcal{H}}}\right)_{\mathbb{R}}^{\vee}$ is called a cone if it is invariant under the natural multiplication action of $\mathbb{R}_{>0}^{\times}$on the $\mathbb{R}$-vector space $\left(\mathbf{S}_{\Phi_{\mathcal{H}}}\right)_{\mathbb{R}}^{\vee}$.

(2) A cone in $\left(\mathbf{S}_{\Phi_{\mathcal{H}}}\right)_{\mathbb{R}}^{\vee}$ is nondegenerate if its closure does not contain any nonzero $\mathbb{R}$-vector subspace of $\left(\mathbf{S}_{\Phi_{\mathcal{H}}}\right)_{\mathbb{R}}^{\vee}$.

(3) A rational polyhedral cone in $\left(\mathbf{S}_{\Phi_{\mathcal{H}}}\right)_{\mathbb{R}}^{\vee}$ is a cone in $\left(\mathbf{S}_{\Phi_{\mathcal{H}}}\right)_{\mathbb{R}}^{\vee}$ of the form $\sigma=$ $\mathbb{R}_{>0} v_{1}+\ldots+\mathbb{R}_{>0} v_{n}$ with $v_{1}, \ldots, v_{n} \in\left(\mathbf{S}_{\Phi_{\mathcal{H}}}\right)_{\mathbb{Q}}^{\vee}=\mathbf{S}_{\Phi_{\mathcal{H}}}^{\vee} \otimes_{\mathbb{Z}} \mathbb{Q}$.

(4) A supporting hyperplane of $\sigma$ is a hyperplane $P$ in $\left(\mathbf{S}_{\Phi_{\mathcal{H}}}\right)_{\mathbb{R}}^{\vee}$ such that $\sigma$ does not overlap with both sides of $P$.

(5) A face of $\sigma$ is a rational polyhedral cone $\tau$ such that $\bar{\tau}=\bar{\sigma} \cap P$ for some supporting hyperplane $P$ of $\sigma$. (Here an overline on a cone means its closure in the ambient space $\left(\mathbf{S}_{\Phi_{\mathcal{H}}}\right)_{\mathbb{R}}^{\vee}$.)

Let $\mathbf{P}_{\Phi_{\mathcal{H}}}$ be the subset of $\left(\mathbf{S}_{\Phi_{\mathcal{H}}}\right)_{\mathbb{R}}^{\vee}$ corresponding to positive semidefinite Hermitian pairings $0 \cdot, \cdot D:\left(Y \otimes_{\mathbb{Z}} \mathbb{R}\right) \times\left(Y \otimes_{\mathbb{Z}} \mathbb{R}\right) \rightarrow B \otimes_{\mathbb{Q}} \mathbb{R}$, with radical (namely the annihilator of the whole space) admissible in the sense that it is the $\mathbb{R}$-span of some admissible submodule $Y^{\prime}$ of $Y$. (We say a submodule $Y^{\prime}$ of $Y$ is admissible if $Y^{\prime} \subset Y$ defines an admissible filtration on $Y$; cf. Definition 1.10. In particular, the quotient $Y / Y^{\prime}$ is also an $\mathcal{O}$-lattice.)

Definition 1.23 [Lan 2008, Definitions 6.2.4.1 and 5.4.1.6]. The group $\Gamma_{\Phi_{\mathcal{H}}}$ is the subgroup of elements $\gamma=\left(\gamma_{X}, \gamma_{Y}\right)$ in $\mathrm{GL}_{\mathcal{O}}(X) \times \mathrm{GL}_{\mathcal{O}}(Y)$ satisfying $\phi=\gamma_{X} \phi \gamma_{Y}$, $\varphi_{-2, \mathcal{H}}={ }^{t} \gamma_{X} \varphi_{-2, \mathcal{H}}$, and $\varphi_{0, \mathcal{H}}=\gamma_{Y} \varphi_{0, \mathcal{H}}$ (if we view the latter two as collections of orbits).

The group $\Gamma_{\Phi_{\mathcal{H}}}$ acts on $\mathbf{S}_{\Phi_{\mathcal{H}}}$, and its induced action preserves the subset $\mathbf{P}_{\Phi_{\mathcal{H}}}$ of $\left(\mathbf{S}_{\Phi_{\mathcal{H}}}\right)_{\mathbb{R}}^{\vee}$. (The group $\Gamma_{\Phi_{\mathcal{H}}}$ is the automorphism group of the torus argument $\Phi_{\mathcal{H}}$. Such automorphism groups show up naturally because torus arguments are only determined up to isomorphism.) 
Definition 1.24 [Lan 2008, Definition 6.1.1.12]. A $\Gamma_{\Phi_{\mathcal{H}}}$-admissible rational polyhedral cone decomposition of $\mathbf{P}_{\Phi_{\mathcal{H}}}$ is a collection $\Sigma=\left\{\sigma_{j}\right\}_{j \in J}$ with some indexing set $J$ such that:

(1) Every $\sigma_{j}$ is a nondegenerate rational polyhedral cone.

(2) $\mathbf{P}_{\Phi_{\mathcal{H}}}$ is the disjoint union of all the $\sigma_{j}$ 's in $\Sigma$. For each $j \in J$, the closure of $\sigma_{j}$ in $\mathbf{P}_{\Phi_{\mathcal{H}}}$ is a disjoint union of $\sigma_{k}$ 's with $k \in J$. In other words, $\mathbf{P}_{\Phi_{\mathcal{H}}}=\bigsqcup_{j \in J} \sigma_{j}$ is a stratification of $\mathbf{P}_{\Phi_{\mathcal{H}}}$.

(3) $\Sigma$ is invariant under the action of $\Gamma_{\Phi_{\mathcal{H}}}$ on $\left(\mathbf{S}_{\Phi_{\mathcal{H}}}\right)_{\mathbb{R}}^{\vee}$, in the sense that $\Gamma_{\Phi_{\mathcal{H}}}$ permutes the cones in $\Sigma$. Under this action, the set $\Sigma / \Gamma_{\Phi_{\mathcal{H}}}$ of $\Gamma_{\Phi_{\mathcal{H}}}$-orbits is finite.

Definition 1.25 [Lan 2008, Definition 6.1.1.13]. A rational polyhedral cone $\sigma$ in $\left(\mathbf{S}_{\Phi_{\mathcal{H}}}\right)_{\mathbb{R}}^{\vee}$ is smooth with respect to the integral structure given by $\mathbf{S}_{\Phi_{\mathcal{H}}}^{\vee}$ if we have $\sigma=\mathbb{R}_{>0} v_{1}+\ldots+\mathbb{R}_{>0} v_{n}$ with $v_{1}, \ldots, v_{n}$ part of a $\mathbb{Z}$-basis of $\mathbf{S}_{\Phi_{\mathcal{H}}}^{\vee}$.

Definition 1.26 [Lan 2008, Definition 6.1.1.14]. A $\Gamma_{\Phi_{\mathcal{H}}}$-admissible smooth rational polyhedral cone decomposition of $\mathbf{P}_{\Phi_{\mathcal{H}}}$ is a $\Gamma_{\Phi_{\mathcal{H}}}$-admissible rational polyhedral cone decomposition $\left\{\sigma_{j}\right\}_{j \in J}$ of $\mathbf{P}_{\Phi_{\mathcal{H}}}$ in which every $\sigma_{j}$ is smooth.

Definition 1.27 [Lan 2008, Definition 7.3.1.1]. Let

$$
\Sigma_{\Phi_{\mathcal{H}}}=\left\{\sigma_{j}\right\}_{j \in J}
$$

be any $\Gamma_{\Phi_{\mathcal{H}}}$-admissible rational polyhedral cone decomposition of $\mathbf{P}_{\Phi_{\mathcal{H}}}$. An (invariant) polarization function on $\mathbf{P}_{\Phi_{\mathcal{H}}}$ for the cone decomposition $\Sigma_{\Phi_{\mathcal{H}}}$ is a $\Gamma_{\Phi_{\mathcal{H}}}$-invariant continuous piecewise linear function $\operatorname{pol}_{\Phi_{\mathcal{H}}}: \mathbf{P}_{\Phi_{\mathcal{H}}} \rightarrow \mathbb{R}_{\geq 0}$ such that:

(1) pol $_{\Phi_{\mathcal{H}}}$ is linear (i.e., coincides with a linear function) on each cone $\sigma_{j}$ in $\Sigma_{\Phi_{\mathcal{H}}}$. (In particular, $\operatorname{pol}_{\Phi_{\mathcal{H}}}(t x)=t \mathrm{pol}_{\Phi_{\mathcal{H}}}(x)$ for any $x \in \mathbf{P}_{\Phi_{\mathcal{H}}}$ and $t \in \mathbb{R}_{\geq 0}$.)

(2) $\operatorname{pol}_{\Phi_{\mathcal{H}}}\left(\left(\mathbf{P}_{\Phi_{\mathcal{H}}} \cap \mathbf{S}_{\Phi_{\mathcal{H}}}^{\vee}\right)-\{0\}\right) \subset \mathbb{Z}_{>0}$. (In particular, pol $\Phi_{\Phi_{\mathcal{H}}}(x)>0$ for any nonzero $x$ in $\mathbf{P}_{\Phi_{\mathcal{H}}}$.)

(3) pol $_{\Phi_{\mathcal{H}}}$ is linear (in the above sense) on a rational polyhedral cone $\sigma$ in $\mathbf{P}_{\Phi_{\mathcal{H}}}$ if and only if $\sigma$ is contained in some cone $\sigma_{j}$ in $\Sigma_{\Phi_{\mathcal{H}}}$.

(4) For any $x, y \in \mathbf{S}_{\Phi_{\mathcal{H}}}$, we have $\operatorname{pol}_{\Phi_{\mathcal{H}}}(x+y) \geq \operatorname{pol}_{\Phi_{\mathcal{H}}}(x)+\operatorname{pol}_{\Phi_{\mathcal{H}}}(y)$. This is called the convexity of $\mathrm{pol}_{\Phi_{\mathcal{H}}}$.

If such a polarization function exists, then we say that the $\Gamma_{\Phi_{\mathcal{H}}}$-admissible rational polyhedral cone decomposition $\Sigma_{\Phi_{\mathcal{H}}}$ is projective.

Definition 1.28. An admissible boundary component of $\mathbf{P}_{\Phi_{\mathcal{H}}}$ is the image of $\mathbf{P}_{\Phi_{\mathcal{H}}^{\prime}}$ under the embedding $\left(\mathbf{S}_{\Phi_{\mathcal{H}}^{\prime}}\right)_{\mathbb{R}}^{\vee} \hookrightarrow\left(\mathbf{S}_{\Phi_{\mathcal{H}}}\right)_{\mathbb{R}}^{\vee}$ defined by some surjection $\left(\Phi_{\mathcal{H}}, \delta_{\mathcal{H}}\right) \rightarrow$ $\left(\Phi_{\mathcal{H}}^{\prime}, \delta_{\mathcal{H}}^{\prime}\right)$. (See Definition 1.19.)

We shall always assume that the following technical condition is satisfied: 
Condition 1.29 (cf. [Faltings and Chai 1990, Chapter IV, Remark 5.8(a)]; see also [Lan 2008, Condition 6.2.5.25 in the revision]). The cone decomposition $\Sigma_{\Phi_{\mathcal{H}}}=\left\{\sigma_{j}\right\}_{j \in J}$ of $\mathbf{P}_{\Phi_{\mathcal{H}}}$ is chosen such that, for any $j \in J$, if $\gamma \bar{\sigma}_{j} \cap \bar{\sigma}_{j} \neq\{0\}$ for some $\gamma \in \Gamma_{\Phi_{\mathcal{H}}}$, then $\gamma$ acts as the identity on the smallest admissible boundary component of $\mathbf{P}_{\Phi_{\mathcal{H}}}$ containing $\sigma_{j}$.

This condition is used to ensure that there are no self-intersections of toroidal boundary strata when the level $\mathcal{H}$ is neat.

To describe the toroidal boundary of $\mathrm{M}_{\mathcal{H}}$, we will need not only cusp labels but also the cones:

Definition 1.30 [Lan 2008, Definition 6.2.6.1]. Let $\left(\Phi_{\mathcal{H}}, \delta_{\mathcal{H}}\right)$ and $\left(\Phi_{\mathcal{H}}^{\prime}, \delta_{\mathcal{H}}^{\prime}\right)$ be two representatives of cusp labels at level $\mathcal{H}$, let $\sigma \subset\left(\mathbf{S}_{\Phi_{\mathcal{H}}}\right)_{\mathbb{R}}^{\vee}$, and let $\sigma^{\prime} \subset\left(\mathbf{S}_{\Phi_{\mathcal{H}}^{\prime}}\right)_{\mathbb{R}}^{\vee}$. We say that the two triples $\left(\Phi_{\mathcal{H}}, \delta_{\mathcal{H}}, \sigma\right)$ and $\left(\Phi_{\mathcal{H}}^{\prime}, \delta_{\mathcal{H}}^{\prime}, \sigma^{\prime}\right)$ are equivalent if there exists a pair of isomorphisms $\gamma=\left(\gamma_{X}: X^{\prime} \stackrel{\sim}{\longrightarrow} X, \gamma_{Y}: Y \stackrel{\sim}{\longrightarrow} Y^{\prime}\right)$ (of $\mathcal{O}$-lattices) such that:

(1) The two representatives $\left(\Phi_{\mathcal{H}}, \delta_{\mathcal{H}}\right)$ and $\left(\Phi_{\mathcal{H}}^{\prime}, \delta_{\mathcal{H}}^{\prime}\right)$ are equivalent under $\gamma$ (as in [Lan 2008, Definition 5.4.2.4], the general level analogue of Definition 1.15).

(2) The isomorphism $\left(\mathbf{S}_{\Phi_{\mathcal{H}}^{\prime}}\right)_{\mathbb{R}}^{\vee} \stackrel{\sim}{\longrightarrow}\left(\mathbf{S}_{\Phi_{\mathcal{H}}}\right)_{\mathbb{R}}^{\vee}$ induced by $\gamma$ sends $\sigma^{\prime}$ to $\sigma$.

In this case, we say that the two triples $\left(\Phi_{\mathcal{H}}, \delta_{\mathcal{H}}, \sigma\right)$ and $\left(\Phi_{\mathcal{H}}^{\prime}, \delta_{\mathcal{H}}^{\prime}, \sigma^{\prime}\right)$ are equivalent under the pair of isomorphisms $\gamma=\left(\gamma_{X}, \gamma_{Y}\right)$.

Definition 1.31 [Lan 2008, Definition 6.2.6.2]. Let $\left(\Phi_{\mathcal{H}}, \delta_{\mathcal{H}}\right)$ and $\left(\Phi_{\mathcal{H}}^{\prime}, \delta_{\mathcal{H}}^{\prime}\right)$ be two representatives of cusp labels at level $\mathcal{H}$, and let $\Sigma_{\Phi_{\mathcal{H}}}$ (resp. $\Sigma_{\Phi_{\mathcal{H}}^{\prime}}$ ) be a $\Gamma_{\Phi_{\mathcal{H}}}$-admissible (resp. $\Gamma_{\Phi_{\mathcal{H}}^{\prime}}$-admissible) smooth rational polyhedral cone decomposition of $\mathbf{P}_{\Phi_{\mathcal{H}}}$ (resp. $\mathbf{P}_{\Phi_{\mathcal{H}}^{\prime}}$ ). We say that the two triples $\left(\Phi_{\mathcal{H}}, \delta_{\mathcal{H}}, \Sigma_{\Phi_{\mathcal{H}}}\right)$ and $\left(\Phi_{\mathcal{H}}^{\prime}, \delta_{\mathcal{H}}^{\prime}, \Sigma_{\Phi_{\mathcal{H}}^{\prime}}\right)$ are equivalent if $\left(\Phi_{\mathcal{H}}, \delta_{\mathcal{H}}\right)$ and $\left(\Phi_{\mathcal{H}}^{\prime}, \delta_{\mathcal{H}}^{\prime}\right)$ are equivalent under some pair of isomorphisms $\gamma=\left(\gamma_{X}: X^{\prime} \stackrel{\sim}{\longrightarrow} X, \gamma_{Y}: Y \stackrel{\sim}{\longrightarrow} Y^{\prime}\right)$, and if under one (and hence every) such $\gamma$ the cone decomposition $\Sigma_{\Phi_{\mathcal{H}}}$ of $\mathbf{P}_{\Phi_{\mathcal{H}}}$ is identified with the cone decomposition $\Sigma_{\Phi_{\mathcal{H}}^{\prime}}$ of $\mathbf{P}_{\Phi_{\mathcal{H}}^{\prime}}$. In this case we say that the two triples $\left(\Phi_{\mathcal{H}}, \delta_{\mathcal{H}}, \Sigma_{\Phi_{\mathcal{H}}}\right)$ and $\left(\Phi_{\mathcal{H}}^{\prime}, \delta_{\mathcal{H}}^{\prime}, \Sigma_{\Phi_{\mathcal{H}}^{\prime}}\right)$ are equivalent under the pair of isomorphisms $\gamma=\left(\gamma_{X}, \gamma_{Y}\right)$.

The compatibility among cone decompositions over different cusp labels is described as follows:

Definition 1.32 [Lan 2008, Definition 6.2.6.4]. Let $\left(\Phi_{\mathcal{H}}, \delta_{\mathcal{H}}\right)$ and $\left(\Phi_{\mathcal{H}}^{\prime}, \delta_{\mathcal{H}}^{\prime}\right)$ be two representatives of cusp labels at level $\mathcal{H}$, and let $\Sigma_{\Phi_{\mathcal{H}}}$ (resp. $\Sigma_{\Phi_{\mathcal{H}}^{\prime}}$ ) be a $\Gamma_{\Phi_{\mathcal{H}}}$-admissible (resp. $\Gamma_{\Phi_{\mathcal{H}}^{\prime}}$-admissible) smooth rational polyhedral cone decomposition of $\mathbf{P}_{\Phi_{\mathcal{H}}}$ (resp. $\mathbf{P}_{\Phi_{\mathcal{H}}^{\prime}}$ ). A surjection $\left(\Phi_{\mathcal{H}}, \delta_{\mathcal{H}}, \Sigma_{\Phi_{\mathcal{H}}}\right) \rightarrow\left(\Phi_{\mathcal{H}}^{\prime}, \delta_{\mathcal{H}}^{\prime}, \Sigma_{\Phi_{\mathcal{H}}^{\prime}}\right.$ ) is given by a surjection $s=\left(s_{X}: X \rightarrow X^{\prime}, s_{Y}: Y \rightarrow Y^{\prime}\right):\left(\Phi_{\mathcal{H}}, \delta_{\mathcal{H}}\right) \rightarrow\left(\Phi_{\mathcal{H}}^{\prime}, \delta_{\mathcal{H}}^{\prime}\right)$ (see Definition 1.19) that induces an embedding $\mathbf{P}_{\Phi_{\mathcal{H}}^{\prime}} \hookrightarrow \mathbf{P}_{\Phi_{\mathcal{H}}}$ such that the restriction $\Sigma_{\Phi_{\mathcal{H}}} \mid \mathbf{P}_{\Phi_{\mathcal{H}}^{\prime}}$ of the cone decomposition $\Sigma_{\Phi_{\mathcal{H}}}$ of $\mathbf{P}_{\Phi_{\mathcal{H}}}$ to $\mathbf{P}_{\Phi_{\mathcal{H}}^{\prime}}$ is the cone decomposition $\Sigma_{\Phi_{\mathcal{H}}^{\prime}}$ of $\mathbf{P}_{\Phi_{\mathcal{H}}^{\prime}}$. 
This allows us to define:

Definition 1.33 [Lan 2008, Condition 6.3.3.1 and Definition 6.3.3.2]. A compatible choice of admissible smooth rational polyhedral cone decomposition data for $\mathrm{M}_{\mathcal{H}}$ is a complete set $\Sigma=\left\{\Sigma_{\Phi_{\mathcal{H}}}\right\}$ of compatible choices of $\Sigma_{\Phi_{\mathcal{H}}}$ (satisfying Condition 1.29) such that, for every surjection $\left(\Phi_{\mathcal{H}}, \delta_{\mathcal{H}}\right) \rightarrow\left(\Phi_{\mathcal{H}}^{\prime}, \delta_{\mathcal{H}}^{\prime}\right)$ of representatives of cusp labels, the cone decompositions $\Sigma_{\Phi_{\mathcal{H}}}$ and $\Sigma_{\Phi_{\mathcal{H}}^{\prime}}$ define a surjection $\left(\Phi_{\mathcal{H}}, \delta_{\mathcal{H}}, \Sigma_{\Phi_{\mathcal{H}}}\right) \rightarrow$ $\left(\Phi_{\mathcal{H}}^{\prime}, \delta_{\mathcal{H}}^{\prime}, \Sigma_{\Phi_{\mathcal{H}}^{\prime}}\right)$ as in Definition 1.32.

Definition 1.34 [Lan 2008, Definition 7.3.1.3]. We say that a compatible choice $\Sigma=\left\{\Sigma_{\Phi_{\mathcal{H}}}\right\}$ of admissible smooth rational polyhedral cone decomposition data for $\mathrm{M}_{\mathcal{H}}$ (see Definition 1.33) is projective if it satisfies the following condition: There is a collection pol $=\left\{\right.$ pol $\left._{\Phi_{\mathcal{H}}}: \mathbf{P}_{\Phi_{\mathcal{H}}} \rightarrow \mathbb{R}_{\geq 0}\right\}$ of polarization functions labeled by representatives $\left(\Phi_{\mathcal{H}}, \delta_{\mathcal{H}}\right)$ of cusp labels, each pol $\Phi_{\mathcal{H}}$ being a polarization function of the cone decomposition $\Sigma_{\Phi_{\mathcal{H}}}$ in $\Sigma$ (see Definition 1.27), which are compatible in the following sense: For any surjection $\left(\Phi_{\mathcal{H}}, \delta_{\mathcal{H}}\right) \rightarrow\left(\Phi_{\mathcal{H}}^{\prime}, \delta_{\mathcal{H}}^{\prime}\right)$ of representatives of cusp labels (see Definition 1.19) inducing an embedding $\mathbf{P}_{\Phi_{\mathcal{H}}^{\prime}} \hookrightarrow \mathbf{P}_{\Phi_{\mathcal{H}}}$, we have $\operatorname{pol}_{\Phi_{\mathcal{H}}} \mid \mathbf{P}_{\Phi_{\mathcal{H}}}=\operatorname{pol}_{\Phi_{\mathcal{H}}^{\prime}}$.

The most important relations among cone decompositions and among compatible choices of them are the so-called refinements:

Definition 1.35 [Lan 2008, Definition 6.2.6.3]. Let $\left(\Phi_{\mathcal{H}}, \delta_{\mathcal{H}}\right)$ and $\left(\Phi_{\mathcal{H}}^{\prime}, \delta_{\mathcal{H}}^{\prime}\right)$ be two representatives of cusp labels at level $\mathcal{H}$, and let $\Sigma_{\Phi_{\mathcal{H}}}\left(\right.$ resp. $\Sigma_{\Phi_{\mathcal{H}}^{\prime}}$ ) be a $\Gamma_{\Phi_{\mathcal{H}}}$-admissible (resp. $\Gamma_{\Phi_{\mathcal{H}}^{\prime}}$-admissible) smooth rational polyhedral cone decomposition of $\mathbf{P}_{\Phi_{\mathcal{H}}}$ (resp. $\mathbf{P}_{\Phi_{\mathcal{H}}^{\prime}}$ ). We say that the triple $\left(\Phi_{\mathcal{H}}, \delta_{\mathcal{H}}, \Sigma_{\Phi_{\mathcal{H}}}\right)$ is a refinement of the triple $\left(\Phi_{\mathcal{H}}^{\prime}, \delta_{\mathcal{H}}^{\prime}, \Sigma_{\Phi_{\mathcal{H}}^{\prime}}\right)$ if $\left(\Phi_{\mathcal{H}}, \delta_{\mathcal{H}}\right)$ and $\left(\Phi_{\mathcal{H}}^{\prime}, \delta_{\mathcal{H}}^{\prime}\right)$ are equivalent under some pair of isomorphisms $\gamma=\left(\gamma_{X}, \gamma_{Y}\right)$, and if under one (and hence every) such $\gamma$ the cone decomposition $\Sigma_{\Phi_{\mathcal{H}}}$ of $\mathbf{P}_{\Phi_{\mathcal{H}}}$ is identified with a refinement of the cone decomposition $\Sigma_{\Phi_{\mathcal{H}}^{\prime}}$ of $\mathbf{P}_{\Phi_{\mathcal{H}}^{\prime}}$. In this case we say that the triple $\left(\Phi_{\mathcal{H}}, \delta_{\mathcal{H}}, \Sigma_{\Phi_{\mathcal{H}}}\right)$ is a refinement of the triple $\left(\Phi_{\mathcal{H}}^{\prime}, \delta_{\mathcal{H}}^{\prime}, \Sigma_{\Phi_{\mathcal{H}}^{\prime}}\right)$ under the pair of isomorphisms $\gamma=\left(\gamma_{X}, \gamma_{Y}\right)$.

Definition 1.36 [Lan 2008, Definition 6.4.2.2]. Let $\Sigma=\left\{\Sigma_{\Phi_{\mathcal{H}}}\right\}$ and $\Sigma^{\prime}=\left\{\Sigma_{\Phi_{\mathcal{H}}}^{\prime}\right\}$ be two compatible choices of admissible smooth rational polyhedral cone decomposition data for $\mathrm{M}_{\mathcal{H}}$. We say that $\Sigma$ refines $\Sigma^{\prime}$ if the triple $\left(\Phi_{\mathcal{H}}, \delta_{\mathcal{H}}, \Sigma_{\Phi_{\mathcal{H}}}\right)$ is a refinement of the triple $\left(\Phi_{\mathcal{H}}, \delta_{\mathcal{H}}, \Sigma_{\Phi_{\mathcal{H}}}^{\prime}\right)$, as in Definition 1.35, for $\left(\Phi_{\mathcal{H}}, \delta_{\mathcal{H}}\right)$ running through all representatives of cusp labels.

Finally, we would like to describe the relations among the equivalence classes $\left[\left(\Phi_{\mathcal{H}}, \delta_{\mathcal{H}}, \sigma\right)\right]$, which will describe the "incidence relations" among (closures of) the toroidal boundary strata.

Definition 1.37 [Lan 2008, Definition 6.3.2.14]. Let $\left(\Phi_{\mathcal{H}}, \delta_{\mathcal{H}}\right)$ be a representative of a cusp label at level $\mathcal{H}$, and let $\sigma \subset \mathbf{P}_{\Phi_{\mathcal{H}}}^{+}$be a nondegenerate smooth rational polyhedral cone. We say that a triple $\left(\Phi_{\mathcal{H}}^{\prime}, \delta_{\mathcal{H}}^{\prime}, \sigma^{\prime}\right)$ is a face of $\left(\Phi_{\mathcal{H}}, \delta_{\mathcal{H}}, \sigma\right)$ if: 
(1) $\left(\Phi_{\mathcal{H}}^{\prime}, \delta_{\mathcal{H}}^{\prime}\right)$ is the representative of some cusp label at level $\mathcal{H}$, such that there exists a surjection $s=\left(s_{X}, s_{Y}\right):\left(\Phi_{\mathcal{H}}, \delta_{\mathcal{H}}\right) \rightarrow\left(\Phi_{\mathcal{H}}^{\prime}, \delta_{\mathcal{H}}^{\prime}\right)$ as in Definition 1.19.

(2) $\sigma^{\prime} \subset \mathbf{P}_{\Phi_{\mathcal{H}}^{\prime}}^{+}$is a nondegenerate smooth rational polyhedral cone, such that for one (and hence every) surjection $s=\left(s_{X}, s_{Y}\right)$ as above, the image of $\sigma^{\prime}$ under the induced embedding $\mathbf{P}_{\Phi_{\mathcal{H}}^{\prime}} \hookrightarrow \mathbf{P}_{\Phi_{\mathcal{H}}}$ is contained in the $\Gamma_{\Phi_{\mathcal{H}}}$-orbit of a face of $\sigma$.

Note that this definition is insensitive to the choices of representatives in the classes $\left[\left(\Phi_{\mathcal{H}}, \delta_{\mathcal{H}}, \sigma\right)\right]$ and $\left[\left(\Phi_{\mathcal{H}}^{\prime}, \delta_{\mathcal{H}}^{\prime}, \sigma^{\prime}\right)\right]$. This justifies the following:

Definition 1.38 [Lan 2008, Definition 6.3.2.15]. We say that the equivalence class $\left[\left(\Phi_{\mathcal{H}}^{\prime}, \delta_{\mathcal{H}}^{\prime}, \sigma^{\prime}\right)\right]$ of $\left(\Phi_{\mathcal{H}}^{\prime}, \delta_{\mathcal{H}}^{\prime}, \sigma^{\prime}\right)$ is a face of the equivalence class $\left[\left(\Phi_{\mathcal{H}}, \delta_{\mathcal{H}}, \sigma\right)\right]$ of $\left(\Phi_{\mathcal{H}}, \delta_{\mathcal{H}}, \sigma\right)$ if some triple equivalent to $\left(\Phi_{\mathcal{H}}^{\prime}, \delta_{\mathcal{H}}^{\prime}, \sigma^{\prime}\right)$ is a face of some triple equivalent to $\left(\Phi_{\mathcal{H}}, \delta_{\mathcal{H}}, \sigma\right)$.

\section{E. Arithmetic toroidal compactifications.}

Definition 1.39 [Lan 2008, Definition 5.3.2.1]. Let $S$ be a normal locally noetherian algebraic stack. A tuple $\left(G, \lambda, i, \alpha_{\mathcal{H}}\right)$ over $S$ is called a degenerating family of type $\mathrm{M}_{\mathcal{H}}$, or simply a degenerating family when the context is clear, if there exists a dense subalgebraic stack $S_{1}$ of $S$, such that $S_{1}$ is defined over $\operatorname{Spec}\left(\mathcal{O}_{F_{0},(\square)}\right)$, and such that:

(1) By viewing group schemes as relative schemes (cf. [Hakim 1972]), $G$ is a semiabelian scheme over $S$ whose restriction $G_{S_{1}}$ to $S_{1}$ is an abelian scheme. In this case, the dual semiabelian scheme $G^{\vee}$ exists (up to unique isomorphism), whose restriction $G_{S_{1}}^{\vee}$ to $S_{1}$ is the dual abelian scheme of $G_{S_{1}}$.

(2) $\lambda: G \rightarrow G^{\vee}$ is a group homomorphism that induces by restriction a prime-to- $\square$ polarization $\lambda_{S_{1}}$ of $G_{S_{1}}$.

(3) $i: \mathcal{O} \rightarrow \operatorname{End}_{S}(G)$ is a homomorphism that defines by restriction an $\mathcal{O}$-structure $i_{S_{1}}: \mathcal{O} \rightarrow \operatorname{End}_{S_{1}}\left(G_{S_{1}}\right)$ of $\left(G_{S_{1}}, \lambda_{S_{1}}\right)$.

(4) $\left(G_{S_{1}}, \lambda_{S_{1}}, i_{S_{1}}, \alpha_{\mathcal{H}}\right) \rightarrow S_{1}$ defines a tuple parametrized by the moduli problem $\mathrm{M}_{\mathcal{H}}$.

We will only talk about (semiabelian) degenerations (of abelian varieties with PEL structures) of this form.

Definition 1.40 [Lan 2008, Definition 6.3.1]. Let $\left(G, \lambda, i, \alpha_{\mathcal{H}}\right)$ be a degenerating family of type $\mathrm{M}_{\mathcal{H}}$ over $S$ (as in Definition 1.39) over $\mathrm{S}_{0}=\operatorname{Spec}\left(\mathcal{O}_{F_{0}}\right.$, $\left.\square\right)$ ). Let $\underline{\mathrm{Lie}}_{G / S}^{\vee}:=e_{G}^{*} \Omega_{G / S}^{1}$ be the dual of $\underline{\mathrm{Lie}}_{G / S}$, and let $\underline{\mathrm{Lie}}_{G^{\vee} / S}^{\vee}:=e_{G^{*}}^{*} \Omega_{G^{\vee} / S}^{1}$ be the dual of $\underline{\mathrm{Lie}}_{G^{\vee} / S}$. Note that $\lambda: G \rightarrow G^{\vee}$ induces an $\mathcal{O}$-equivariant morphism $\lambda^{*}: \underline{\mathrm{Lie}}_{G^{\vee} / S}^{\vee} \rightarrow \underline{\mathrm{Lie}}_{G / S}^{\vee}$. (Here the $\mathcal{O}$-action on $\underline{\mathrm{Lie}}_{G / S}^{\vee}$ is a left action after twisting by the involution $\star$.) Then we define the sheaf $\underline{\mathrm{KS}}=\underline{\mathrm{KS}}(G, \lambda) / S=\underline{\mathrm{KS}}\left(G, \lambda, i, \alpha_{\mathcal{H}}\right) / S$ by 
setting

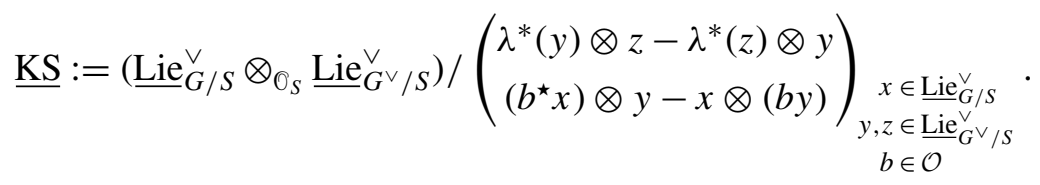

Analogues of the sheaf $\underline{\mathrm{KS}}$ appear naturally in the deformation theory of abelian varieties with PEL structures (without degenerations). The point of Definition 1.40 is that it extends the conventional definition (for abelian schemes with PEL structures) to the context of (semiabelian) degenerating families (see Definition 1.39).

Theorem 1.41 [Lan 2008, Theorems 6.4.1.1 and 7.3.3.4]. To each compatible choice $\Sigma=\left\{\Sigma_{\Phi_{\mathcal{H}}}\right\}$ of admissible smooth rational polyhedral cone decomposition data as in Definition 1.33, there is associated a proper smooth algebraic stack $\mathrm{M}_{\mathcal{H}, \Sigma}^{\text {tor }}$ over $\mathrm{S}_{0}=\operatorname{Spec}\left(\mathcal{O}_{F_{0},(\square)}\right)$, which is an algebraic space when $\mathcal{H}$ is neat (as in Definition 1.2), containing $\mathrm{M}_{\mathcal{H}}$ as an open dense subalgebraic stack, together with a degenerating family $\left(G, \lambda, i, \alpha_{\mathcal{H}}\right)$ over $\mathrm{M}_{\mathcal{H}}^{\text {tor }}$ (as in Definition 1.39) such that:

(1) The restriction $\left(G_{\mathrm{M}_{\mathcal{H}}}, \lambda_{\mathrm{M}_{\mathcal{H}}}, i_{\mathrm{M}_{\mathcal{H}}}, \alpha_{\mathcal{H}}\right)$ of the degenerating family $\left(G, \lambda, i, \alpha_{\mathcal{H}}\right)$ to $\mathrm{M}_{\mathcal{H}}$ is the tautological (i.e., universal) tuple over $\mathrm{M}_{\mathcal{H}}$.

(2) $\mathrm{M}_{\mathcal{H}}^{\text {tor }}$ has a stratification by locally closed subalgebraic stacks

$$
\mathrm{M}_{\mathcal{H}, \Sigma}^{\text {tor }}=\coprod_{\left[\left(\Phi_{\mathcal{H}}, \delta_{\mathcal{H}}, \sigma\right)\right]} \mathrm{Z}_{\left[\left(\Phi_{\mathcal{H}}, \delta_{\mathcal{H}}, \sigma\right)\right]},
$$

with $\left[\left(\Phi_{\mathcal{H}}, \delta_{\mathcal{H}}, \sigma\right)\right]$ running through a complete set of equivalence classes of $\left(\Phi_{\mathcal{H}}, \delta_{\mathcal{H}}, \sigma\right)$ (as in Definition 1.30) with $\sigma \subset \mathbf{P}_{\Phi_{\mathcal{H}}}^{+}$and $\sigma \in \Sigma_{\Phi_{\mathcal{H}}} \in \Sigma$. (Here $\mathrm{Z}_{\mathcal{H}}$ is suppressed in the notation by our convention.)

In this stratification, the $\left[\left(\Phi_{\mathcal{H}}^{\prime}, \delta_{\mathcal{H}}^{\prime}, \sigma^{\prime}\right)\right]$-stratum $\mathrm{Z}_{\left[\left(\Phi_{\mathcal{H}}^{\prime}, \delta_{\mathcal{H}}^{\prime}, \sigma^{\prime}\right)\right]}$ lies in the closure of the $\left[\left(\Phi_{\mathcal{H}}, \delta_{\mathcal{H}}, \sigma\right)\right]$-stratum $\mathrm{Z}_{\left[\left(\Phi_{\mathcal{H}}, \delta_{\mathcal{H}}, \sigma\right)\right]}$ if and only if $\left[\left(\Phi_{\mathcal{H}}, \delta_{\mathcal{H}}, \sigma\right)\right]$ is a face of $\left[\left(\Phi_{\mathcal{H}}^{\prime}, \delta_{\mathcal{H}}^{\prime}, \sigma^{\prime}\right)\right]$ as in Definition 1.38.

The $\left[\left(\Phi_{\mathcal{H}}, \delta_{\mathcal{H}}, \sigma\right)\right]$-stratum $\mathrm{Z}_{\left[\left(\Phi_{\mathcal{H}}, \delta_{\mathcal{H}}, \sigma\right)\right]}$ is smooth and isomorphic to the support of the formal algebraic stack $\mathfrak{X}_{\Phi_{\mathcal{H}}, \delta_{\mathcal{H}}, \sigma} / \Gamma_{\Phi_{\mathcal{H}}, \sigma}$ for any representative $\left(\Phi_{\mathcal{H}}, \delta_{\mathcal{H}}, \sigma\right)$ of $\left[\left(\Phi_{\mathcal{H}}, \delta_{\mathcal{H}}, \sigma\right)\right]$, where the formal algebraic stack $\mathfrak{X}_{\Phi_{\mathcal{H}}, \delta_{\mathcal{H}}, \sigma}$ (before quotient by $\Gamma_{\Phi_{\mathcal{H}}, \sigma}$, the subgroup of $\Gamma_{\Phi_{\mathcal{H}}}$ formed by elements mapping $\sigma$ to itself) admits a canonically defined structure of a torus-torsor over an abelian scheme over the smooth algebraic stack $\mathrm{M}_{\mathcal{H}}^{Z_{\mathcal{H}}}$ in Definition 1.17. (Note that $\mathrm{Z}_{\mathcal{H}}$ and the isomorphism class of $\mathrm{M}_{\mathcal{H}}^{\mathrm{Z}_{\mathcal{H}}}$ depend only on the class $\left[\left(\Phi_{\mathcal{H}}, \delta_{\mathcal{H}}, \sigma\right)\right]$, but not on the choice of the representative $\left(\Phi_{\mathcal{H}}, \delta_{\mathcal{H}}, \sigma\right)$.)

In particular, $\mathrm{M}_{\mathcal{H}}$ is an open dense stratum in this stratification.

(3) The complement of $\mathrm{M}_{\mathcal{H}}$ in $\mathrm{M}_{\mathcal{H}, \Sigma}^{\text {tor }}$ (with its reduced structure) is a relative Cartier divisor $\mathrm{D}_{\infty, \mathcal{H}}$ with normal crossings, such that each connected component of a stratum of $\mathrm{M}_{\mathcal{H}}^{\text {tor }}-\mathrm{M}_{\mathcal{H}}$ is open dense in an intersection of irreducible components 
of $\mathrm{D}_{\infty, \mathcal{H}}$ (including possible self-intersections). When $\mathcal{H}$ is neat, the irreducible components of $\mathrm{D}_{\infty, \mathcal{H}}$ have no self-intersections (cf. Condition 1.29, [Lan 2008, Remark 6.2.5.26 in the revision], and [Faltings and Chai 1990, Chapter IV, Remark 5.8(a)]).

(4) The extended Kodaira-Spencer morphism [Lan 2008, Definition 4.6.3.32] for $G \rightarrow \mathrm{M}_{\mathcal{H}}^{\text {tor }}$ induces an isomorphism

$$
\mathrm{KS}_{G / \mathrm{M}_{\mathcal{H}}^{\text {tor }} / \mathrm{S}_{0}}: \underline{\mathrm{KS}}_{G / \mathrm{M}_{\mathcal{H}}^{\text {tor }}} \stackrel{\sim}{\longrightarrow} \Omega_{\mathrm{M}_{\mathcal{H}}^{\text {tor }} / \mathrm{S}_{0}}^{1}[d \log \infty]
$$

(see Definition 1.40). Here the sheaf $\Omega_{\mathrm{M}_{\mathcal{H}}^{\text {tor }} / \mathrm{S}_{0}}[d \log \infty]$ is the sheaf of modules of log 1-differentials on $\mathrm{M}_{\mathcal{H}}^{\text {tor }}$ over $\mathrm{S}_{0}$, with respect to the relative Cartier divisor $\mathrm{D}_{\infty, \mathcal{H}}$ with normal crossings.

(5) The formal completion

$$
\left(\mathrm{M}_{\mathcal{H}}^{\mathrm{tor}}\right)_{\mathrm{Z}_{\left[\left(\Phi_{\mathcal{H}}, \delta_{\mathcal{H}}, \sigma\right)\right]}}
$$

of $\mathrm{M}_{\mathcal{H}}^{\text {tor }}$ along the $\left[\left(\Phi_{\mathcal{H}}, \delta_{\mathcal{H}}, \sigma\right)\right]$-stratum $\mathrm{Z}_{\left[\left(\Phi_{\mathcal{H}}, \delta_{\mathcal{H}}, \sigma\right)\right]}$ is canonically isomorphic to the formal algebraic stack $\mathfrak{X}_{\Phi_{\mathcal{H}}, \delta_{\mathcal{H}}, \sigma} / \Gamma_{\Phi_{\mathcal{H}}, \sigma}$ for any representative $\left(\Phi_{\mathcal{H}}, \delta_{\mathcal{H}}, \sigma\right)$ of $\left[\left(\Phi_{\mathcal{H}}, \delta_{\mathcal{H}}, \sigma\right)\right]$. (To form the formal completion along a given locally closed stratum, we first remove the other strata appearing in the closure of this stratum from the total space, and then form the formal completion of the remaining space along this stratum.)

This isomorphism respects stratifications in the sense that, given any formally étale morphism $\operatorname{Spf}(R, I) \rightarrow \mathfrak{X}_{\Phi_{\mathcal{H}}, \delta_{\mathcal{H}}, \sigma} / \Gamma_{\Phi_{\mathcal{H}}, \sigma}$ inducing a morphism $\operatorname{Spec}(R) \rightarrow \Xi_{\Phi_{\mathcal{H}}, \delta_{\mathcal{H}}}(\sigma) / \Gamma_{\Phi_{\mathcal{H}}, \sigma}$, the stratification of $\operatorname{Spec}(R)$ (inherited from $\Xi_{\Phi_{\mathcal{H}}, \delta_{\mathcal{H}}}(\sigma) / \Gamma_{\Phi_{\mathcal{H}}, \sigma}$; see [Lan 2008, Proposition 6.3.1.6 and Definition 6.3.2.16 in the revision]) makes the induced morphism $\operatorname{Spec}(R) \rightarrow \mathrm{M}_{\mathcal{H}}^{\text {tor }}$ a stratapreserving morphism.

The pullback to $\left(\mathrm{M}_{\mathcal{H}}^{\text {tor }}\right)_{\hat{\mathrm{Z}}_{\left[\left(\Phi_{\mathcal{H}}, \delta_{\mathcal{H}}, \sigma\right)\right]}}$ of the degenerating family $\left(G, \lambda, i, \alpha_{\mathcal{H}}\right)$ over $\mathrm{M}_{\mathcal{H}}^{\text {tor }}$ is the Mumford family

$$
\left({ }^{\circ} G,{ }^{\circ} \lambda,{ }^{\circ} i,{ }^{\circ} \alpha_{\mathcal{H}}\right)
$$

over $\mathfrak{X}_{\Phi_{\mathcal{H}}, \delta_{\mathcal{H}}, \sigma} / \Gamma_{\Phi_{\mathcal{H}}, \sigma}$ (see [Lan 2008, §6.2.5]) after we identify the bases using the isomorphism. (Here both the pullback of $\left(G, \lambda, i, \alpha_{\mathcal{H}}\right)$ and the Mumford family $\left({ }^{\circ} G,{ }^{\circ} \lambda,{ }^{\circ} i,{ }^{\circ} \alpha_{\mathcal{H}}\right)$ are considered as relative schemes with additional structures; cf. [Hakim 1972].)

(6) Let $S$ be an irreducible noetherian normal scheme over $\mathrm{S}_{0}$. Suppose we have a degenerating family $\left(G^{\dagger}, \lambda^{\dagger}, i^{\dagger}, \alpha_{\mathcal{H}}^{\dagger}\right)$ of type $\mathrm{M}_{\mathcal{H}}$ over $S$ as in Definition 1.39. Then $\left(G^{\dagger}, \lambda^{\dagger}, i^{\dagger}, \alpha_{\mathcal{H}}^{\dagger}\right) \rightarrow S$ is the pullback of $\left(G, \lambda, i, \alpha_{\mathcal{H}}\right) \rightarrow \mathrm{M}_{\mathcal{H}}^{\text {tor }}$ via a (necessarily unique) morphism $S \rightarrow \mathrm{M}_{\mathcal{H}}^{\text {tor }}$ (over $\mathrm{S}_{0}$ ) if and only if the following condition is satisfied: 
Consider any dominant morphism $\operatorname{Spec}(V) \rightarrow S$ centered at a geometric point $\bar{s}$ of $S$, where $V$ is a complete discrete valuation ring with quotient field $K$, algebraically closed residue field $k$, and discrete valuation $v$. Let

$$
\left(G^{\ddagger}, \lambda^{\ddagger}, i^{\ddagger}, \alpha_{\mathcal{H}}^{\ddagger}\right) \rightarrow \operatorname{Spec}(V)
$$

be the pullback of $\left(G^{\dagger}, \lambda^{\dagger}, i^{\dagger}, \alpha_{\mathcal{H}}^{\dagger}\right) \rightarrow S$. This pullback family defines an object of $\mathrm{DEG}_{\mathrm{PEL}, \mathrm{M}_{\mathcal{H}}}$ over $\operatorname{Spec}(V)$, which corresponds to a tuple

$$
\left(A^{\ddagger}, \lambda_{A}^{\ddagger}, i_{A}^{\ddagger}, \underline{X}^{\ddagger}, \underline{Y}^{\ddagger}, \phi^{\ddagger}, c^{\ddagger},\left(c^{\vee}\right)^{\ddagger}, \tau^{\ddagger},\left[\left(\alpha_{\mathcal{H}}^{\natural}\right)^{\ddagger}\right]\right)
$$

in $\mathrm{DD}_{\mathrm{PEL}, \mathrm{M}_{\mathcal{H}}}$ (under [Lan 2008, Theorem 5.3.1.17]). Then we have a fully symplectic-liftable admissible filtration $\mathrm{Z}_{\mathcal{H}}^{\ddagger}$ determined by $\left[\left(\alpha_{\mathcal{H}}^{\natural}\right)^{\ddagger}\right]$. Moreover, the étale sheaves $\underline{X}^{\ddagger}$ and $\underline{Y}^{\ddagger}$ are necessarily constant, because the base ring $V$ is strict local. Hence it makes sense to say we also have a uniquely determined torus argument $\Phi_{\mathcal{H}}^{\ddagger}$ at level $\mathcal{H}$ for $\mathrm{Z}_{\mathcal{H}}^{\ddagger}$.

On the other hand, we have objects $\underline{\Phi}_{\mathcal{H}}\left(G^{\ddagger}\right), \underline{\mathbf{S}}_{\Phi_{\mathcal{H}}\left(G^{\ddagger}\right)}$, and $\underline{B}\left(G^{\ddagger}\right)$ (see [Lan 2008, Construction 6.3.1.1]), which define objects $\Phi_{\mathcal{H}}^{\ddagger}, \mathbf{S}_{\Phi_{\mathcal{H}}^{\ddagger}}$ and in particular $B^{\dagger}: \mathbf{S}_{\Phi_{\mathcal{H}}^{+}} \rightarrow \operatorname{Inv}(V)$ over the special fiber. Then

$$
v \circ B^{\ddagger}: \mathbf{S}_{\Phi_{\mathcal{H}}^{\ddagger}} \rightarrow \mathbb{Z}
$$

defines an element of $\mathbf{S}_{\Phi_{H}^{\ddagger}}^{\vee}$, where $v: \operatorname{Inv}(V) \rightarrow \mathbb{Z}$ is the homomorphism induced by the discrete valuation of $V$.

Then the condition is that, for any $\operatorname{Spec}(V) \rightarrow S$ as above, and for any choice of $\delta_{\mathcal{H}}$ (which is immaterial, because this choice will not be used), there is a cone $\sigma^{\ddagger}$ in the cone decomposition $\Sigma_{\Phi_{\mathcal{H}}^{\ddagger}}$ of $\mathbf{P}_{\Phi_{\mathcal{H}}^{\ddagger}}$ (given by the choice of $\Sigma$; cf. Definition 1.33) such that $\bar{\sigma}^{\ddagger}$ contains all the $\mathcal{v} \circ B^{\ddagger}$ obtained in this way.

(7) If $\mathcal{H}$ is neat and $\Sigma$ is projective (see Definition 1.34), then $\mathrm{M}_{\mathcal{H}, \Sigma}^{\text {tor }}$ is projective (and hence a scheme) over $\mathrm{S}_{0}$.

Statement (1) means the tautological tuple over $\mathrm{M}_{\mathcal{H}}$ extends to a degenerating family $\left(G, \lambda, i, \alpha_{\mathcal{H}}\right)$ over $\mathrm{M}_{\mathcal{H}}^{\text {tor }}$. (Since $\mathrm{M}_{\mathcal{H}}^{\text {tor }}$ is normal, this extension is unique by a result of Raynaud; see [Raynaud 1970, IX, 1.4] or [Faltings and Chai 1990, Chapter I, Proposition 2.7].) Statements (2)-(5) and (7) are self-explanatory. Statement (6) can be interpreted as a "universal property" for the degenerating family $\left(G, \lambda, i, \alpha_{\mathcal{H}}\right) \rightarrow \mathrm{M}_{\mathcal{H}}^{\text {tor }}$ among degenerating families over normal locally noetherian bases, as in Definition 1.39, satisfying moreover some conditions describing the "degenerating patterns" over pullbacks to complete discrete valuation rings with algebraically closed residue fields. This "universal property" will be crucial in the main construction of this article (in Section 3A below). 


\section{Kuga families and their compactifications}

Let $\mathcal{O},{ }^{\star},(L,\langle\cdot, \cdot\rangle), h$, and $\square$ be as in the previous section. Then we have a moduli problem $\mathrm{M}_{\mathcal{H}}$ over $\mathrm{S}_{0}=\operatorname{Spec}\left(\mathcal{O}_{F_{0},(\square)}\right)$ for each open compact $\mathcal{H}$ of $\mathrm{G}\left(\hat{\mathbb{Z}}^{\square}\right)$, with a toroidal compactification $\mathrm{M}_{\mathcal{H}, \Sigma}^{\text {tor }}$ for each choice of $\Sigma$.

For simplicity, let us maintain the following:

Convention 2.1. All morphisms between schemes or algebraic stacks over $\mathrm{S}_{0}=$ $\operatorname{Spec}\left(\mathcal{O}_{F_{0},(\square)}\right)$ will be defined over $\mathrm{S}_{0}$, unless otherwise specified.

2A. PEL-type Kuga families. Let $Q$ be any $\mathcal{O}$-lattice. Consider the abelian scheme $G_{\mathrm{M}_{\mathcal{H}}}$ over $\mathrm{M}_{\mathcal{H}}$ in (1) of Theorem 1.41. By [Lan 2008, Proposition 5.2.3.8], the group functor $\underline{\operatorname{Hom}}_{\mathcal{O}}\left(Q, G_{\mathrm{M}_{\mathcal{H}}}\right)$ over $\mathrm{M}_{\mathcal{H}}$ is representable by a proper smooth group scheme which is an extension of a finite étale group scheme, whose rank has no prime factors other than those of Disc, by an abelian scheme $\underline{\operatorname{Hom}}_{\mathcal{O}}\left(Q, G_{\mathrm{M}_{\mathcal{H}}}\right)^{\circ}$, which we call the fiberwise geometric identity component of $\underline{\operatorname{Hom}}_{\mathcal{O}}\left(Q, G_{\mathrm{M}_{\mathcal{H}}}\right)$.

Example 2.2. If $Q \cong \mathcal{O}^{\oplus s}$ for some integer $s \geq 0$, then $\underline{\operatorname{Hom}}_{\mathcal{O}}\left(Q, G_{\mathrm{M}_{\mathcal{H}}}\right)^{\circ}=$ $\underline{\operatorname{Hom}}_{\mathcal{O}}\left(Q, G_{\mathrm{M}_{\mathcal{H}}}\right) \cong G_{\mathrm{M}_{\mathcal{H}}}^{\times s}$ is the $s$-fold fiber product of $G_{\mathrm{M}_{\mathcal{H}}}$ over $\mathrm{M}_{\mathcal{H}}$.

Example 2.3. If $\mathcal{O} \cong \mathrm{M}_{k}\left(\mathcal{O}_{F}\right)$ and $Q$ is of finite index in $\mathcal{O}_{F}^{\oplus k}$ for some integer $k \geq 1$, then the relative dimension of $\underline{\operatorname{Hom}}_{\mathcal{O}}\left(Q, G_{\mathrm{M}_{\mathcal{H}}}\right)^{\circ}$ over $\mathrm{M}_{\mathcal{H}}$ is $1 / k$ of the relative dimension of $G_{\mathrm{M}_{\mathcal{H}}}$ over $\mathrm{M}_{\mathcal{H}}$.

Definition 2.4. A PEL-type Kuga family over $\mathrm{M}_{\mathcal{H}}$ is an abelian scheme $\mathrm{N} \rightarrow \mathrm{M}_{\mathcal{H}}$ that is $\mathbb{Z}_{(\square)}^{\times}$-isogenous to $\underline{\operatorname{Hom}}_{\mathcal{O}}\left(Q, G_{\mathrm{M}_{\mathcal{H}}}\right)^{\circ}$ for some $\mathcal{O}$-lattice $Q$.

Consider Diff ${ }^{-1}=\operatorname{Diff}_{\mathcal{O} / \mathbb{Z}}^{-1}$, the inverse different of $\mathcal{O}$ over $\mathbb{Z}[$ Lan 2008, Definition 1.1.1.11] with its canonical left $\mathcal{O}$-module structure. Since the trace pairing Diff $^{-1} \times \mathcal{O} \rightarrow \mathbb{Z}:(y, x) \mapsto \operatorname{Tr}_{\mathcal{O} / \mathbb{Z}}(y x)$ is perfect by definition, for each $\mathcal{O}$-lattice $Q$, we may identify $Q^{\vee}:=\operatorname{Hom}_{\mathbb{Z}}(Q, \mathbb{Z})$ with $\operatorname{Hom}_{\mathcal{O}}\left(Q, \operatorname{Diff}^{-1}\right)$. By composition with the involution ${ }^{\star}: \mathcal{O} \sim \mathcal{O}^{\text {op }}$, the natural right action of $\mathcal{O}$ on Diff $^{-1}$ induced a left action of $\mathcal{O}$ on Diff ${ }^{-1}$, which commutes with the natural left action of $\mathcal{O}$ on Diff ${ }^{-1}$. Accordingly, the $\mathbb{Z}$-module $Q^{\vee}$ is torsion-free and has a canonical left $\mathcal{O}$-structure induced by the right action of $\mathcal{O}^{\text {op }}$ on $\operatorname{Diff}^{-1}$ (and ${ }^{\star}: \mathcal{O} \simeq \mathcal{O}^{\text {op }}$ ). In other words, $Q^{\vee}$ is an $\mathcal{O}$-lattice. Then the trace pairing induces a perfect pairing

$$
\langle\cdot, \cdot\rangle_{Q}: Q^{\vee} \times Q \rightarrow \mathbb{Z}:(f, x) \mapsto \operatorname{Tr}_{\mathcal{O} / \mathbb{Z}}(f(x)) .
$$

For any $b \in \mathcal{O}, f \in Q^{\vee}$, and $x \in Q$, we have

$$
\langle b f, x\rangle_{Q}=\operatorname{Tr}_{\mathcal{O} / \mathbb{Z}}\left(f(x) b^{\star}\right)=\operatorname{Tr}_{\mathcal{O} / \mathbb{Z}}\left(b^{\star} f(x)\right)=\operatorname{Tr}_{\mathcal{O} / \mathbb{Z}}\left(f\left(b^{\star} x\right)\right)=\left\langle f, b^{\star} x\right\rangle .
$$

Lemma 2.5. There exists an embedding $j_{Q}: Q^{\vee} \hookrightarrow Q$ of $\mathcal{O}$-lattices inducing an isomorphism $j_{Q}: Q^{\vee} \otimes_{\mathbb{Z}} \mathbb{Z}_{(\square)} \stackrel{\sim}{\rightarrow} Q \otimes_{\mathbb{Z}} \mathbb{Z}_{(\square)}$ of $\mathcal{O} \otimes_{\mathbb{Z}} \mathbb{Z}_{(\square)}$-modules such that the 
pairing

$$
\left\langle j_{Q}^{-1}(\cdot), \cdot\right\rangle_{Q}:\left(Q \otimes_{\mathbb{Z}} \mathbb{R}\right) \times\left(Q \otimes_{\mathbb{Z}} \mathbb{R}\right) \rightarrow \mathbb{R}
$$

is positive definite.

Proof. By the explicit classification [Lan 2008, (1.2.1.10), Proposition 1.2.1.13, and Lemma 1.2.1.23], there exists an isomorphism $j_{Q, 0}: Q^{\vee} \otimes_{\mathbb{Z}} \mathbb{R} \sim Q \otimes_{\mathbb{Z}} \mathbb{R}$ of $\mathcal{O} \otimes_{\mathbb{Z}}$ $\mathbb{R}$-modules such that the induced pairing $\left\langle j_{Q, 0}^{-1}(\cdot), \cdot\right\rangle_{Q}:\left(Q \otimes_{\mathbb{Z}} \mathbb{R}\right) \times\left(Q \otimes_{\mathbb{Z}} \mathbb{R}\right) \rightarrow \mathbb{R}$ is positive definite. If $\square$ is the set of all rational prime numbers, then necessarily $\mathcal{O}=\mathbb{Z}$, and the lemma is clear. Otherwise, we know that $\operatorname{Isom}_{\mathcal{O} \otimes_{\mathbb{Z}} \mathbb{Z}_{(\square)}}\left(Q^{\vee} \otimes_{\mathbb{Z}} \mathbb{Z}_{(\square)}, Q \otimes_{\mathbb{Z}}\right.$ $\left.\mathbb{Z}_{(\square)}\right)$ is dense in $\operatorname{Isom}_{\mathcal{O} \otimes_{\mathbb{Z}} \mathbb{R}}\left(Q^{\vee} \otimes_{\mathbb{Z}} \mathbb{R}, Q \otimes_{\mathbb{Z}} \mathbb{R}\right)$ (with the topology induced by $\mathbb{R})$. Hence there exists an element $j_{Q, 1}: Q^{\vee} \otimes_{\mathbb{Z}} \mathbb{Z}_{(\square)} \stackrel{\sim}{\rightarrow} Q \otimes_{\mathbb{Z}} \mathbb{Z}_{(\square)}$ close to $j_{Q, 0}$ in $\operatorname{Isom}_{\mathcal{O} \otimes_{\mathbb{Z}} \mathbb{R}}\left(Q^{\vee} \otimes_{\mathbb{Z}} \mathbb{R}, Q \otimes_{\mathbb{Z}} \mathbb{R}\right)$ such that the induced pairing $\left\langle j_{Q, 1}^{-1}(\cdot), \cdot\right\rangle_{Q}$ : $\left(Q \otimes_{\mathbb{Z}} \mathbb{R}\right) \times\left(Q \otimes_{\mathbb{Z}} \mathbb{R}\right) \rightarrow \mathbb{R}$ is still positive definite. By multiplying $j_{Q, 1}$ by a positive element in $\mathbb{Z}_{(\square)}^{\times}$, we may assume that it maps $Q^{\vee}$ to a submodule of $Q$. Then the induced morphism $j_{Q}: Q^{\vee} \rightarrow Q$ satisfies the requirement of the lemma.

Lemma 2.6. The abelian scheme $\underline{\operatorname{Hom}}_{\mathbb{Z}}\left(Q^{\vee}, G_{\mathrm{M}_{\mathcal{H}}}^{\vee}\right)$ is isomorphic to the dual abelian scheme of $\underline{\operatorname{Hom}}_{\mathbb{Z}}\left(Q, G_{\mathrm{M}_{\mathcal{H}}}\right)$.

Proof. Let $s$ be the common rank of $Q$ and $Q^{\vee}$ as free $\mathbb{Z}$-modules. Let $\left\{e_{1}, \ldots, e_{s}\right\}$ be a $\mathbb{Z}$-basis of $Q$, and let $\left\{e_{1}^{\vee}, \ldots, e_{s}^{\vee}\right\}$ be the dual $\mathbb{Z}$-basis of $Q^{\vee}$, such that $e_{i}^{\vee}\left(e_{j}\right)=\delta_{i j}$ for any $1 \leq i, j \leq s$. Then the choices of bases define canonical isomorphisms

$$
\underline{\operatorname{Hom}}_{\mathbb{Z}}\left(Q, G_{\mathrm{M}_{\mathcal{H}}}\right) \cong G_{\mathrm{M}_{\mathcal{H}}}^{\times s}
$$

and

$$
\underline{\operatorname{Hom}}_{\mathbb{Z}}\left(Q^{\vee}, G_{\mathrm{M}_{\mathcal{H}}}^{\vee}\right) \cong\left(G_{\mathrm{M}_{\mathcal{H}}}^{\vee}\right)^{\times s} .
$$

As a result, $\underline{\operatorname{Hom}}_{\mathbb{Z}}\left(Q^{\vee}, G_{\mathrm{M}_{\mathcal{H}}}^{\vee}\right) \cong G_{\mathrm{M}_{\mathcal{H}}}^{\times s}$ is isomorphic to the dual abelian scheme of $\underline{\operatorname{Hom}}_{\mathbb{Z}}\left(Q, G_{\mathrm{M}_{\mathcal{H}}}\right) \cong\left(G_{\mathrm{M}_{\mathcal{H}}}^{\vee}\right)^{\times s}$.

Lemma 2.9. Let $j_{Q}: Q^{\vee} \hookrightarrow Q$ be as in Lemma 2.5. Then the isogeny

$$
\lambda_{\mathrm{M}_{\mathcal{H}}, j_{Q}, \mathbb{Z}}: \underline{\operatorname{Hom}_{\mathbb{Z}}}\left(Q, G_{\mathrm{M}_{\mathcal{H}}}\right) \rightarrow \underline{\operatorname{Hom}_{\mathbb{Z}}}\left(Q^{\vee}, G_{\mathrm{M}_{\mathcal{H}}}^{\vee}\right)
$$

induced canonically by $j_{Q}$ and $\lambda_{\mathrm{M}_{\mathcal{H}}}: G_{\mathrm{M}_{\mathcal{H}}} \rightarrow G_{\mathrm{M}_{\mathcal{H}}}^{\vee}$, which is of degree prime to $\square$ because both $\left[Q: j_{Q}\left(Q^{\vee}\right)\right]$ and $\operatorname{deg}\left(\lambda_{\mathrm{M}_{\mathcal{H}}}\right)$ are prime to $\square$, is a polarization.

Proof. We need to show that the invertible sheaf

$$
\left.\left(\operatorname{Id}_{\underline{\mathrm{Hom}_{\mathbb{Z}}}}\left(Q, G_{\mathrm{M}_{\mathcal{H}}}\right), \lambda_{\mathrm{M}_{\mathcal{H}}, j_{Q}, \mathbb{Z}}\right)^{*} \mathcal{P}_{\underline{\mathrm{Hom}_{\mathbb{Z}}}\left(Q, G_{\mathrm{M}_{\mathcal{H}}}\right.}\right)
$$

is relative ample over $\mathrm{M}_{\mathcal{H}}$. Using the choice of basis $\left\{e_{1}, \ldots, e_{s}\right\}$ (resp. $\left.\left\{e_{1}^{\vee}, \ldots, e_{s}^{\vee}\right\}\right)$ of $Q$ (resp. $Q^{\vee}$ ) as in the proof of Lemma 2.6, the morphism $j_{Q}$ can be represented by $e_{i}^{\vee} \mapsto \sum_{1 \leq j \leq s} a_{i j} e_{j}$ for some integers $a_{i j}$, for each $1 \leq i \leq s$. These integers form 
a positive definite matrix $a=\left(a_{i j}\right)$, because the induced pairing $\left\langle j_{Q}^{-1}(\cdot), \cdot\right\rangle_{Q}$ : $\left(Q \otimes_{\mathbb{Z}} \mathbb{R}\right) \times\left(Q \otimes_{\mathbb{Z}} \mathbb{R}\right) \rightarrow \mathbb{R}$ is positive definite. By completion of squares for quadratic forms, we know that there exist an integer $m \geq 1$ such that $m a=u d^{t} u$ for some matrices $d$ and $u$ with integral coefficients, where $d=\operatorname{diag}\left(d_{1}, \ldots, d_{s}\right)$ is diagonal with positive entries. As a result, the morphism $m \lambda_{M_{\mathcal{H}}, j_{Q}, \mathbb{Z}}$ factors as a composition

$$
m \lambda_{\mathrm{M}_{\mathcal{H}}, j_{Q}, \mathbb{Z}}=\left[{ }^{t} u\right]^{*} \circ \lambda_{\mathrm{M}_{\mathcal{H}}, d, \mathbb{Z}} \circ[u]^{*}
$$

of morphisms

$$
\begin{aligned}
& {[u]^{*}: \underline{\operatorname{Hom}}_{\mathbb{Z}}\left(Q, G_{\mathrm{M}_{\mathcal{H}}}\right) \rightarrow \underline{\operatorname{Hom}}_{\mathbb{Z}}\left(Q, G_{\mathrm{M}_{\mathcal{H}}}\right),} \\
& \lambda_{\mathrm{M}_{\mathcal{H}}, d, \mathbb{Z}}: \underline{\operatorname{Hom}}_{\mathbb{Z}}\left(Q, G_{\mathrm{M}_{\mathcal{H}}}\right) \rightarrow \underline{\operatorname{Hom}}_{\mathbb{Z}}\left(Q^{\vee}, G_{\mathrm{M}_{\mathcal{H}}}^{\vee}\right), \\
& {\left[{ }^{t} u\right]^{*}: \underline{\operatorname{Hom}}_{\mathbb{Z}}\left(Q^{\vee}, G_{\mathrm{M}_{\mathcal{H}}}^{\vee}\right) \rightarrow \underline{\operatorname{Hom}}_{\mathbb{Z}}\left(Q^{\vee}, G_{\mathrm{M}_{\mathcal{H}}}^{\vee}\right) \text {. }}
\end{aligned}
$$

If we identify $\underline{\operatorname{Hom}}_{\mathbb{Z}}\left(Q, G_{\mathrm{M}_{\mathcal{H}}}\right)$ and $\underline{\operatorname{Hom}}_{\mathbb{Z}}\left(Q^{\vee}, G_{\mathrm{M}_{\mathcal{H}}}^{\vee}\right)$ as dual abelian schemes of each other using the canonical isomorphisms (2.7) and (2.8) defined by the dual bases $\left\{e_{1}, \ldots, e_{s}\right\}$ and $\left\{e_{1}^{\vee}, \ldots, e_{s}^{\vee}\right\}$, then $\left[{ }^{t} u\right]^{*}=\left([u]^{*}\right)^{\vee}$, and $\lambda_{\mathrm{M}_{\mathcal{H}}, d, \mathbb{Z}}=$ $\left(d_{1} \lambda_{\mathrm{M}_{\mathcal{H}}}\right) \times\left(d_{2} \lambda_{\mathrm{M}_{\mathcal{H}}}\right) \times \ldots \times\left(d_{s} \lambda_{\mathrm{M}_{\mathcal{H}}}\right): G_{\mathrm{M}_{\mathcal{H}}}^{\times s} \rightarrow\left(G_{\mathrm{M}_{\mathcal{H}}}^{\vee}\right)^{\times s}$ is a polarization. Since $[u]^{*}$ is finite, this implies that $\lambda_{\mathrm{M}_{\mathcal{H}}, j_{Q}, \mathbb{Z}}$ is also a polarization, as desired.

Proposition 2.10. The abelian scheme $\underline{\operatorname{Hom}}_{\mathcal{O}}\left(Q^{\vee}, G_{\mathrm{M}_{\mathcal{H}}}^{\vee}\right)^{\circ}$ is $\mathbb{Z}_{(\square)}^{\times}$-isogenous to the dual abelian scheme of $\underline{\operatorname{Hom}}_{\mathcal{O}}\left(Q, G_{\mathrm{M}_{\mathcal{H}}}\right)^{\circ}$.

Proof. Since $\lambda_{\mathrm{M}_{\mathcal{H}}, j_{Q}, \mathbb{Z}}$ is a polarization by Lemma 2.9 , the induced morphism

$$
\begin{aligned}
\lambda_{\mathrm{M}_{\mathcal{H}}, j_{Q}}: \underline{\operatorname{Hom}_{\mathcal{O}}}\left(Q, G_{\mathrm{M}_{\mathcal{H}}}\right)^{\circ} \hookrightarrow & \underline{\operatorname{Hom}_{\mathbb{Z}}}\left(Q, G_{\mathrm{M}_{\mathcal{H}}}\right) \\
\stackrel{\lambda_{\mathrm{M}_{\mathcal{H}}, j_{Q}, \mathbb{Z}}}{\rightarrow} & \underline{\operatorname{Hom}_{\mathbb{Z}}}\left(Q^{\vee}, G_{\mathrm{M}_{\mathcal{H}}}^{\vee}\right) \rightarrow\left(\underline{\left.\operatorname{Hom}_{\mathcal{O}}\left(Q, G_{\mathrm{M}_{\mathcal{H}}}\right)^{\circ}\right)^{\vee}}\right.
\end{aligned}
$$

is also a polarization. (Since the condition of being a polarization can be checked fiber by fiber [Deligne and Pappas 1994, 1.2-1.4], it suffices to note that the restriction of an ample invertible sheaf to a closed subscheme is again ample.)

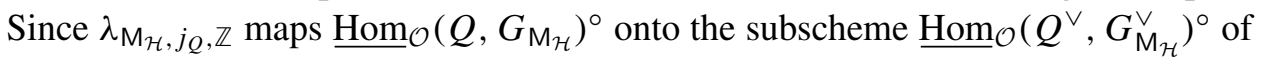
$\underline{\operatorname{Hom}}_{\mathbb{Z}}\left(Q^{\vee}, G_{\mathrm{M}_{\mathcal{H}}}^{\vee}\right)$, we obtain an isogeny

$$
\underline{\operatorname{Hom}}_{\mathcal{O}}\left(Q^{\vee}, G_{\mathrm{M}_{\mathcal{H}}}^{\vee}\right)^{\circ} \rightarrow\left(\underline{\operatorname{Hom}}_{\mathcal{O}}\left(Q, G_{\mathrm{M}_{\mathcal{H}}}\right)^{\circ}\right)^{\vee}
$$

The degree of this isogeny is prime to $\square$ because $\lambda_{\mathrm{M}_{\mathcal{H}}, j_{Q}, \mathbb{Z}}$ is.

Corollary 2.12 (of the proof of Proposition 2.10). Let $j_{Q}: Q^{\vee} \hookrightarrow Q$ be as in Lemma 2.5. Then the canonical morphism

$$
\lambda_{\mathrm{M}_{\mathcal{H}}, j_{Q}}: \underline{\operatorname{Hom}}_{\mathcal{O}}\left(Q, G_{\mathrm{M}_{\mathcal{H}}}\right)^{\circ} \rightarrow\left(\underline{\operatorname{Hom}}_{\mathcal{O}}\left(Q, G_{\mathrm{M}_{\mathcal{H}}}\right)^{\circ}\right)^{\vee}
$$

induced by $j_{Q}$ and $\lambda_{\mathrm{M}_{\mathcal{H}}}: G_{\mathrm{M}_{\mathcal{H}}} \rightarrow G_{\mathrm{M}_{\mathcal{H}}}^{\vee}$ (as in (2.11)) is a polarization of degree prime to $\square$. 
Corollary 2.13. If a Kuga family $\mathrm{N} \rightarrow \mathrm{M}_{\mathcal{H}}$ is $\mathbb{Z}_{(\square)}^{\times}$-isogenous to $\underline{\operatorname{Hom}}_{\mathcal{O}}\left(Q, G_{\mathrm{M}_{\mathcal{H}}}\right)^{\circ}$ for some $\mathcal{O}$-lattice $Q$, then we have canonical isomorphisms over $\mathrm{M}_{\mathcal{H}}$ :

$$
\begin{aligned}
& \underline{\operatorname{Lie}}_{\mathrm{N} / \mathrm{M}_{\mathcal{H}}} \cong \underline{\operatorname{Hom}}_{\mathcal{O}}\left(Q, \underline{\operatorname{Lie}}_{G / \mathrm{M}_{\mathcal{H}}}\right), \quad \underline{\operatorname{Lie}}_{\mathrm{N}^{\vee} / \mathrm{M}_{\mathcal{H}}} \cong \underline{\operatorname{Hom}}_{\mathcal{O}}\left(Q^{\vee}, \underline{\operatorname{Lie}}_{G^{\vee}} / \mathrm{M}_{\mathcal{H}}\right), \\
& \underline{\operatorname{Lie}}_{\mathrm{N} / \mathrm{M}_{\mathcal{H}}}^{\vee} \cong \underline{\operatorname{Hom}}_{\mathcal{O}}\left(Q^{\vee}, \underline{\operatorname{Lie}}_{G / \mathrm{M}_{\mathcal{H}}}^{\vee}\right), \quad \underline{\operatorname{Lie}}_{\mathrm{N}^{\vee} / \mathrm{M}_{\mathcal{H}}}^{\vee} \underline{\operatorname{Hom}}_{\mathcal{O}}\left(Q, \underline{\operatorname{Lie}}_{G^{\vee} / \mathrm{M}_{\mathcal{H}}}\right) \text {. }
\end{aligned}
$$

Remark 2.14. We do not need to choose a polarization $N \rightarrow N^{\vee}$ in the isomorphisms in Corollary 2.13. The sheaves on the right-hand sides of the isomorphisms are locally free because the order $\mathcal{O}$ is maximal at any good prime (see Definition 1.5 and [Lan 2008, Proposition 1.1.1.17]), and because lattices over maximal orders are projective modules (see [Lan 2008, Proposition 1.1.1.20]).

2B. Main theorem. (Convention 2.1 will persist until the end of this article.)

Theorem 2.15. Let $Q$ be any $\mathcal{O}$-lattice. Suppose that $\mathcal{H}$ is neat (as in Definition 1.2), so that the moduli problem $\mathrm{M}_{\mathcal{H}}$ it defines is representable by a quasiprojective scheme, and so that $\mathrm{M}_{\mathcal{H}}^{\text {tor }}=\mathrm{M}_{\mathcal{H}, \Sigma}^{\text {tor }}$ is a proper smooth algebraic space over $\mathrm{S}_{0}$. Then there is a directed partially ordered set $\mathbf{K}_{Q, \mathcal{H}, \Sigma}$ parametrizing the following data:

(1) For each $\kappa \in \mathbf{K}_{Q, \mathcal{H}, \Sigma}$, there is a $\mathbb{Z}_{(\square)}^{\times}$-isogeny $\kappa^{\text {isog }}: \underline{\operatorname{Hom}}_{\mathcal{O}}\left(Q, G_{\mathrm{M}_{\mathcal{H}}}\right)^{\circ} \rightarrow \mathrm{N}_{\kappa}$ over $\mathrm{M}_{\mathcal{H}}$, together with an open immersion $\kappa^{\text {tor }}: \mathrm{N}_{\kappa} \hookrightarrow \mathrm{N}_{\kappa}^{\text {tor }}$ of schemes over $\mathrm{S}_{0}$, such that the scheme $\mathrm{N}_{\kappa}^{\text {tor }}$ is projective and smooth over $\mathrm{S}_{0}$, and that the complement of $\mathrm{N}_{\kappa}$ in $\mathrm{N}_{\kappa}^{\text {tor }}$ (with its reduced structure) is a relative Cartier divisor $\mathrm{E}_{\infty, \kappa}$ with simple normal crossings.

For each relation $\kappa^{\prime} \succ \kappa$ in $\mathbf{K}_{Q, \mathcal{H}, \Sigma}$, there is a proper log étale morphism $f_{\kappa^{\prime}, \kappa}^{\text {tor }}: \mathrm{N}_{\kappa^{\prime}}^{\text {tor }} \rightarrow \mathrm{N}_{\kappa}^{\text {tor }}$ extending the canonical $\mathbb{Z}_{(\square)}^{\times}$-isogeny

$$
f_{\kappa^{\prime}, \kappa}:=\kappa^{\text {isog }} \circ\left(\left(\kappa^{\prime}\right)^{\text {isog }}\right)^{-1}: \mathrm{N}_{\kappa^{\prime}} \rightarrow \mathrm{N}_{\kappa}
$$

such that $R^{i}\left(f_{\kappa^{\prime}, \kappa}^{\text {tor }}\right)_{*} \mathrm{O}_{\mathrm{N}_{\kappa^{\prime}}}^{\text {tor }}=0$ for $i>0$.

(2) For each $\kappa \in \mathbf{K}_{Q, \mathcal{H}, \Sigma}$, the structural morphism $f_{\kappa}: \mathrm{N}_{\kappa} \rightarrow \mathrm{M}_{\mathcal{H}}$ extends (necessarily uniquely) to a morphism $f_{\kappa}^{\text {tor }}: \mathrm{N}_{\kappa}^{\text {tor }} \rightarrow \mathrm{M}_{\mathcal{H}}^{\text {tor }}$, which is proper and log smooth (as in [Kato 1989, 3.3] and [Illusie 1994, 1.6]) if we equip $\mathrm{N}_{\kappa}^{\text {tor }}$ and $\mathrm{M}_{\mathcal{H}}^{\mathrm{tor}}$ with the canonical (fine) log structures given respectively by the relative Cartier divisors with (simple) normal crossings $\mathrm{E}_{\infty, \kappa}$ and $\mathrm{D}_{\infty, \mathcal{H}}$ (see (1) above and (3) of Theorem 1.41). Then we have the following commutative diagram:

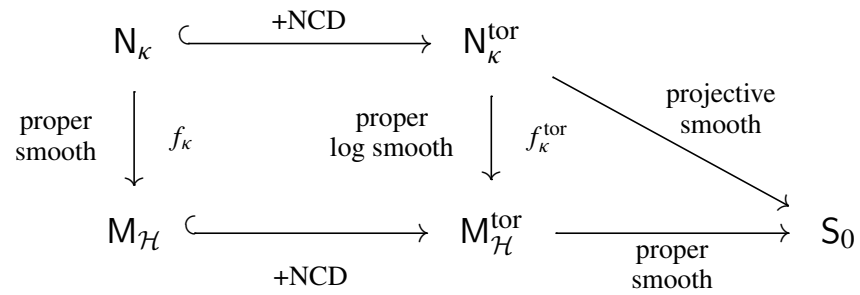

If $\kappa^{\prime} \succ \kappa$, then we have the compatibility $f_{\kappa^{\prime}}^{\text {tor }}=f_{\kappa}^{\text {tor }} \circ f_{\kappa^{\prime}, \kappa}^{\text {tor }}$. 
(3) Let us fix a choice of $\kappa \in \mathbf{K}_{Q, \mathcal{H}, \Sigma}$ and suppress the subscript $\kappa$ from the notation. (All canonical isomorphisms will be required to be compatible with the canonical isomorphisms defined by pullback under $f_{\kappa^{\prime}, \kappa}^{\text {tor }}$ for each relation $\kappa^{\prime} \succ \kappa$.) Then the following are true:

(3a) Let $\Omega_{\mathrm{N}^{\text {tor }} / \mathrm{S}_{0}}^{1}[d \log \infty]$ and $\Omega_{\mathrm{M}_{\mathcal{H}}^{\text {tor }} / \mathrm{S}_{0}}^{1}[d \log \infty]$ denote the sheaves of modules of $\log$ 1-differentials over $\mathrm{S}_{0}$ given by the (respective) canonical log structures defined in (2). Let

$$
\bar{\Omega}_{\mathrm{N}^{\text {tor }} / \mathrm{M}_{\mathcal{H}}^{\text {tor }}}^{1}:=\left(\Omega_{\mathrm{N}^{\text {tor }} / \mathrm{S}_{0}}^{1}[d \log \infty]\right) /\left(\left(f^{\text {tor }}\right)^{*}\left(\Omega_{\mathrm{M}_{\mathcal{H}}^{\text {tor }} / \mathrm{S}_{0}}^{1}[d \log \infty]\right)\right) .
$$

Then there is a canonical isomorphism

$$
\left(f^{\text {tor }}\right)^{*}\left(\underline{\operatorname{Hom}}_{\mathcal{O}}\left(Q^{\vee}, \underline{\operatorname{Lie}}_{G / \mathrm{M}_{\mathcal{H}}^{\text {tor }}}\right)\right) \cong \bar{\Omega}_{\mathrm{N}^{\text {tor }} / \mathrm{M}_{\mathcal{H}}^{\text {tor }}}
$$

between locally free sheaves over $\mathrm{N}^{\text {tor }}$, extending the composition of canonical isomorphisms

$$
f^{*}\left(\underline{\operatorname{Hom}}_{\mathcal{O}}\left(Q^{\vee},{\underline{\operatorname{Lie}_{G}}}_{G_{\mathrm{M}_{\mathcal{H}}} / \mathrm{M}_{\mathcal{H}}}\right)\right) \cong f^{*} \underline{\operatorname{Lie}}_{\mathrm{N} / \mathrm{M}_{\mathcal{H}}}^{\vee} \cong \Omega_{\mathrm{N} / \mathrm{M}_{\mathcal{H}}}^{1}
$$

over $\mathrm{N}$.

(3b) For any integer $b \geq 0$, there exists a canonical isomorphism

$$
\begin{aligned}
& R^{b} f_{*}^{\text {tor }}\left(\bar{\Omega}_{\mathrm{N}^{\text {tor }} / \mathrm{M}_{\mathcal{H}}^{\text {tor }}}\right) \\
& \cong\left(\Lambda^{\vee}\left(\underline{\operatorname{Hom}}_{\mathcal{O}}\left(Q^{\vee},{\underline{\text { Lie }_{G}}}_{G^{\vee} / \mathrm{M}_{\mathcal{H}}^{\text {tor }}}\right)\right)\right) \otimes_{\mathcal{O}_{\mathrm{M}_{\mathcal{H}}^{\text {tor }}}}\left(\bigwedge^{a}\left(\underline{\operatorname{Hom}}_{\mathcal{O}}\left(Q^{\vee},{\underline{\mathrm{Lie}_{G}^{\vee}}}_{G / \mathrm{M}_{\mathcal{H}}^{\text {tor }}}\right)\right)\right) .
\end{aligned}
$$

of locally free sheaves over $\mathrm{M}_{\mathcal{H}}^{\mathrm{tor}}$, compatible with cup products and exterior products, extending the canonical isomorphism over $\mathrm{M}_{\mathcal{H}}$ induced by the composition of canonical isomorphisms

$$
R^{b} f_{*}\left(O_{\mathrm{N}}\right) \cong \Lambda^{b}{\underline{\mathrm{Lie}_{\mathrm{N}}}}_{\mathrm{N}^{\mathrm{M}}} \cong \Lambda^{b}\left(\underline{\operatorname{Hom}}_{\mathcal{O}}\left(Q^{\vee}, \underline{\operatorname{Lie}}_{G_{\mathrm{M}_{\mathcal{H}}} / \mathrm{M}_{\mathcal{H}}}\right)\right)
$$

(3c) Let $\bar{\Omega}_{\mathbf{N}^{\text {tor }} / \mathrm{M}_{\mathcal{H}}^{\text {tor }}}:=\bigwedge^{\bullet} \bar{\Omega}_{\mathrm{N}^{\text {tor }} / \mathrm{M}_{\mathcal{H}}^{\text {tor }}}^{1}$ be the log de Rham complex associated with $f^{\text {tor }}: \mathrm{N}^{\text {tor }} \rightarrow \mathrm{M}_{\mathcal{H}}^{\text {tor }}$ (with differentials inherited from $\Omega_{\mathrm{N} / \mathrm{M}_{\mathcal{H}}}$ ). Let the (relative) log de Rham cohomology be defined by

$$
\underline{H}_{\log -\mathrm{dR}}^{i}\left(\mathrm{~N}^{\text {tor }} / \mathrm{M}_{\mathcal{H}}^{\text {tor }}\right):=R^{i} f_{*}^{\text {tor }}\left(\bar{\Omega}_{\mathrm{N}^{\text {tor }} / \mathrm{M}_{\mathcal{H}}^{\text {tor }}}\right) .
$$

Then the (relative) Hodge spectral sequence

$$
E_{1}^{a, b}:=R^{b} f_{*}^{\text {tor }}\left(\bar{\Omega}_{\mathrm{N}^{\text {tor }} / \mathrm{M}_{\mathcal{H}}^{\text {tor }}}^{a}\right) \Rightarrow \underline{H}_{\log -\mathrm{dR}}^{a+b}\left(\mathrm{~N}^{\text {tor }} / \mathrm{M}_{\mathcal{H}}^{\text {tor }}\right)
$$

degenerates at $E_{1}$ terms, and defines a Hodge filtration on $\underline{H}_{\log -\mathrm{dR}}^{i}\left(\mathrm{~N}^{\text {tor }} / \mathrm{M}_{\mathcal{H}}^{\text {tor }}\right)$ with locally free graded pieces given by $R^{b} f_{*}^{\text {tor }}\left(\bar{\Omega}_{\mathrm{N}^{\mathrm{tor}} / \mathrm{M}_{\mathcal{H}}^{\text {tor }}}^{a}\right)$ for integers $a+b=i$, extending the canonical Hodge filtration on $\underline{H}_{\mathrm{dR}}^{i}\left(\mathrm{~N} / \mathrm{M}_{\mathcal{H}}\right)$.

As a result, for any integer $i \geq 0$, there is a canonical isomorphism

$$
\bigwedge^{i} \underline{H}_{\log -\mathrm{dR}}^{1}\left(\mathrm{~N}^{\text {tor }} / \mathrm{M}_{\mathcal{H}}^{\text {tor }}\right) \stackrel{\sim}{\longrightarrow} \underline{H}_{\log -\mathrm{dR}}^{i}\left(\mathrm{~N}^{\text {tor }} / \mathrm{M}_{\mathcal{H}}^{\text {tor }}\right),
$$


compatible with the Hodge filtrations defined by (2.20), extending the canonical isomorphism $\wedge^{i} \underline{H}_{\mathrm{dR}}^{1}\left(\mathrm{~N} / \mathrm{M}_{\mathcal{H}}\right) \stackrel{\sim}{\longrightarrow} \underline{H}_{\mathrm{dR}}^{i}\left(\mathrm{~N} / \mathrm{M}_{\mathcal{H}}\right)$ over $\mathrm{M}_{\mathcal{H}}$ (defined by cup product).

(3d) For any $j_{Q}: Q^{\vee} \hookrightarrow Q$ as in Lemma 2.5 , the $\mathbb{Z}_{(\square)}^{\times}$-polarization $\lambda_{\mathrm{M}_{\mathcal{H}}, j_{Q}}$ : $\underline{\operatorname{Hom}}_{\mathcal{O}}\left(Q, G_{\mathrm{M}_{\mathcal{H}}}\right)^{\circ} \rightarrow\left(\underline{\operatorname{Hom}}_{\mathcal{O}}\left(Q, G_{\mathrm{M}_{\mathcal{H}}}\right)^{\circ}\right)^{\vee}$ in Corollary 2.12 defines canonically (as in [Deligne and Pappas 1994, 1.5]) a perfect pairing

$$
\langle\cdot, \cdot\rangle_{\lambda_{\mathrm{M}_{\mathcal{H}}, j_{Q}}}: \underline{H}_{\mathrm{dR}}^{1}\left(\mathrm{~N} / \mathrm{M}_{\mathcal{H}}\right) \times \underline{H}_{\mathrm{dR}}^{1}\left(\mathrm{~N} / \mathrm{M}_{\mathcal{H}}\right) \rightarrow \mathrm{O}_{\mathrm{M}_{\mathcal{H}}}(1) .
$$

Then $\underline{H}_{\log -\mathrm{dR}}^{1}\left(\mathrm{~N}^{\text {tor }} / \mathrm{M}_{\mathcal{H}}^{\text {tor }}\right)$ is the unique subsheaf of $\left(\mathrm{M}_{\mathcal{H}} \hookrightarrow \mathrm{M}_{\mathcal{H}}^{\text {tor }}\right)_{*}\left(\underline{H}_{\mathrm{dR}}^{1}\left(\mathrm{~N} / \mathrm{M}_{\mathcal{H}}\right)\right)$ satisfying the following conditions:

(i) $\underline{H}_{\log -\mathrm{dR}}^{1}\left(\mathrm{~N}^{\text {tor }} / \mathrm{M}_{\mathcal{H}}^{\text {tor }}\right)$ is locally free of finite rank over $\mathrm{O}_{\mathrm{M}_{\mathcal{H}}^{\text {tor }}}$.

(ii) The sheaf $f_{*}^{\text {tor }}\left(\bar{\Omega}_{\mathrm{N}^{\text {tor }} / \mathrm{M}_{\mathcal{H}}^{\text {tor }}}\right)$ can be identified as the subsheaf of

$$
\left(\mathrm{M}_{\mathcal{H}} \hookrightarrow \mathrm{M}_{\mathcal{H}}^{\text {tor }}\right)_{*}\left(f_{*}\left(\Omega_{\mathrm{N} / \mathrm{M}_{\mathcal{H}}}^{1}\right)\right)
$$

formed (locally) by sections that are also sections of $\underline{H}_{\log -\mathrm{dR}}^{1}\left(\mathrm{~N}^{\text {tor }} / \mathrm{M}_{\mathcal{H}}^{\text {tor }}\right)$. (Here we are viewing all these sheaves canonically as subsheaves of $\left(\mathrm{M}_{\mathcal{H}} \hookrightarrow \mathrm{M}_{\mathcal{H}}^{\text {tor }}\right)_{*}\left(\underline{H}_{\mathrm{dR}}^{1}\left(\mathrm{~N} / \mathrm{M}_{\mathcal{H}}\right)\right)$. $)$

(iii) $\underline{H}_{\log -\mathrm{dR}}^{1}\left(\mathrm{~N}^{\mathrm{tor}} / \mathrm{M}_{\mathcal{H}}^{\mathrm{tor}}\right)$ is self-dual under the push-forward

$$
\left(\mathrm{M}_{\mathcal{H}} \hookrightarrow \mathrm{M}_{\mathcal{H}}^{\text {tor }}\right)_{*}\langle\cdot, \cdot\rangle_{\lambda_{\mathrm{M}_{\mathcal{H}}, j_{Q}}} .
$$

(3e) The Gauss-Manin connection

$$
\nabla: \underline{H}_{\mathrm{dR}}^{\cdot}\left(\mathrm{N} / \mathrm{M}_{\mathcal{H}}\right) \rightarrow \underline{H}_{\mathrm{dR}}^{\cdot}\left(\mathrm{N} / \mathrm{M}_{\mathcal{H}}\right) \otimes_{\mathrm{O}_{\mathrm{M}_{\mathcal{H}}}} \Omega_{\mathrm{M}_{\mathcal{H}} / \mathrm{S}_{0}}^{1}
$$

extends to an integrable connection

$$
\nabla: \underline{H}_{\log -\mathrm{dR}}\left(\mathrm{N}^{\text {tor }} / \mathrm{M}_{\mathcal{H}}^{\text {tor }}\right) \rightarrow \underline{H}_{\log -\mathrm{dR}}\left(\mathrm{N}^{\text {tor }} / \mathrm{M}_{\mathcal{H}}^{\text {tor }}\right) \otimes_{\mathcal{O}_{\mathrm{M}_{\mathcal{H}}}} \bar{\Omega}_{\mathrm{M}_{\mathcal{H}}^{\text {tor }} / \mathrm{S}_{0}}^{1}
$$

with log poles along $\mathrm{D}_{\infty, \mathcal{H}}$, called the extended Gauss-Manin connection, satisfying the usual Griffith transversality with the Hodge filtration defined by (2.20).

(4) (Hecke actions.) Suppose we have an element $g_{h} \in \mathrm{G}\left(\mathbb{A}^{\infty, \square}\right)$, and suppose we have a (neat) open compact subgroup $\mathcal{H}^{\prime}$ of $\mathrm{G}\left(\hat{\mathbb{Z}}^{\square}\right)$ such that $g_{h}^{-1} \mathcal{H}^{\prime} g_{h} \subset \mathcal{H}$. Suppose $\Sigma^{\prime}=\left\{\Sigma_{\Phi_{\mathcal{H}^{\prime}}^{\prime}}^{\prime}\right\}$ is a compatible choice of admissible smooth rational polyhedral cone decomposition data for $\mathrm{M}_{\mathcal{H}^{\prime}}$, which $g_{h^{\prime}}$-refines $\Sigma$ (as in [Lan 2008, Definition 6.4.3.3]; the notion was called "dominance" in the original version, but changed to the more common "refinement" in the revision). Then there is also a directed partially ordered set $\mathbf{K}_{Q, \mathcal{H}^{\prime}, \Sigma^{\prime}}$ parametrizing (for $\kappa^{\prime} \in$ $\left.\mathbf{K}_{Q, \mathcal{H}^{\prime}, \Sigma^{\prime}}\right) \mathbb{Z}_{(\square)}^{\times}$-isogenies $\underline{\operatorname{Hom}}_{\mathcal{O}}\left(Q, G_{\mathrm{M}_{\mathcal{H}^{\prime}}}\right)^{\circ} \rightarrow \mathrm{N}_{\kappa^{\prime}}^{\prime}$ over $\mathrm{M}_{\mathcal{H}^{\prime}}$, together with open immersions $\mathrm{N}_{\kappa^{\prime}}^{\prime} \hookrightarrow\left(\mathrm{N}_{\kappa^{\prime}}^{\prime}\right)^{\text {tor }}$ of schemes over $\mathrm{S}_{0}$, satisfying analogues of properties (1)-(3) above. The constructions of $\mathbf{K}_{Q, \mathcal{H}, \Sigma}$ and $\mathbf{K}_{Q, \mathcal{H}^{\prime}, \Sigma^{\prime}}$ (and the 
objects they parametrize) satisfy the compatibility with $g_{h}$ in the sense that, for each $\kappa \in \mathbf{K}_{Q, \mathcal{H}, \Sigma}$, there is an element $\kappa^{\prime} \in \mathbf{K}_{Q, \mathcal{H}^{\prime}, \Sigma^{\prime}}$ such that the following are true:

(4a) There exists a (necessarily unique) finite étale morphism $\left[g_{h}\right]_{\kappa^{\prime}, \kappa}: \mathrm{N}_{\kappa^{\prime}}^{\prime} \rightarrow \mathrm{N}_{\kappa}$ covering the morphism $\left[g_{h}\right]: \mathrm{M}_{\mathcal{H}^{\prime}} \rightarrow \mathrm{M}_{\mathcal{H}}$ given by [Lan 2008, Proposition 6.4.3.4], inducing a prime-to- $\square$ isogeny $\mathrm{N}_{\kappa^{\prime}}^{\prime} \rightarrow \mathrm{N}_{\kappa} \times \mathrm{M}_{\mathcal{H}} \mathrm{M}_{\mathcal{H}^{\prime}}$, which agrees with the $\mathbb{Z}_{(\square)}^{\times}$-isogeny induced by $\left(\kappa^{\prime}\right)^{\mathrm{isog}}, \kappa^{\mathrm{isog}}$, and the $\mathbb{Z}_{(\square)}^{\times}$-isogeny $G_{\mathrm{M}_{\mathcal{H}^{\prime}}} \rightarrow G_{\mathrm{M}_{\mathcal{H}}} \times{ }_{\mathrm{M}_{\mathcal{H}}} \mathrm{M}_{\mathcal{H}^{\prime}}$ realizing $G_{\mathrm{M}_{\mathcal{H}}} \times \mathrm{M}_{\mathcal{H}} \mathrm{M}_{\mathcal{H}^{\prime}}$ as a Hecke twist of $G_{\mathrm{M}_{\mathcal{H}^{\prime}}}$ by $g_{h}$. (Here all the base changes from $\mathrm{M}_{\mathcal{H}}$ to $\mathrm{M}_{\mathcal{H}^{\prime}}$ use the morphism $\left[g_{h}\right]$.)

(4b) There exists a (necessarily unique) proper log étale morphism

$$
\left[g_{h}\right]_{\kappa^{\prime}, \kappa}^{\text {tor }}:\left(\mathrm{N}_{\kappa^{\prime}}^{\prime}\right)^{\text {tor }} \rightarrow \mathrm{N}_{\kappa}^{\text {tor }}
$$

extending the morphism $\left[g_{h}\right]_{\kappa^{\prime}, \kappa}$ and covering the morphism $\left[g_{h}\right]^{\text {tor }}: \mathrm{M}_{\mathcal{H}^{\prime}, \Sigma^{\prime}}^{\text {tor }} \rightarrow$ $\mathrm{M}_{\mathcal{H}, \Sigma}^{\text {tor }}$ given by [Lan 2008, Proposition 6.4.3.4], such that

$$
R^{i}\left(\left[g_{h}\right]_{\kappa^{\prime}, \kappa}^{\mathrm{tor}}\right)_{*} \mathrm{O}_{\left(\mathrm{N}_{\kappa^{\prime}}\right)^{\mathrm{tor}}}=0
$$

for any $i>0$.

(4c) There is a canonical isomorphism

$$
\left(\left[g_{h}\right]^{\text {tor }}\right)^{*} \underline{H}_{\log -\mathrm{dR}}^{a+b}\left(\mathrm{~N}_{\kappa}^{\text {tor }} / \mathrm{M}_{\mathcal{H}, \Sigma}^{\text {tor }}\right) \stackrel{\sim}{\longrightarrow} \underline{H}_{\log -\mathrm{dR}}^{a+b}\left(\left(\mathrm{~N}_{\kappa^{\prime}}^{\prime}\right)^{\text {tor }} / \mathrm{M}_{\mathcal{H}^{\prime}, \Sigma^{\prime}}^{\text {tor }}\right)
$$

respecting the Hodge filtrations and compatible with the canonical isomorphisms

$$
\begin{aligned}
& \left(\left[g_{h}\right]_{\kappa^{\prime}, \kappa}^{\text {tor }}\right)^{*} \bar{\Omega}_{\mathrm{N}_{\kappa}^{\text {tor }} / \mathrm{M}_{\mathcal{H}, \Sigma}^{\text {tor }}}^{1} \stackrel{\sim}{\longrightarrow} \bar{\Omega}_{\left(\mathrm{N}_{\kappa^{\prime}}^{\prime}\right)^{\text {tor }} / \mathrm{M}_{\mathcal{H}^{\prime}, \Sigma^{\prime}}^{\text {tor }}}^{1}, \\
& \left(\left[g_{h}\right]^{\text {tor }}\right)^{*} \underline{\mathrm{Lie}}_{G^{\vee} / \mathrm{M}_{\mathcal{H}, \Sigma}^{\text {tor }}} \stackrel{\sim}{\longrightarrow} \underline{\mathrm{Lie}}_{G^{\vee} / \mathrm{M}_{\mathcal{H}^{\prime}, \Sigma^{\prime}}^{\text {tor }}}, \\
& \left(\left[g_{h}\right]^{\text {tor }}\right)^{*} \underline{\mathrm{Lie}}_{G / \mathrm{M}_{\mathcal{H}, \Sigma}^{\mathrm{tor}}}^{\vee} \stackrel{\sim}{\longrightarrow} \underline{\mathrm{Lie}}_{G / \mathrm{M}_{\mathcal{H}^{\prime}, \Sigma^{\prime}}^{\mathrm{tor}},},
\end{aligned}
$$

and the canonical isomorphisms in (3) for $\mathrm{N}_{\kappa}^{\text {tor }}$ and $\left(\mathrm{N}_{\kappa^{\prime}}^{\prime}\right)^{\text {tor }}$.

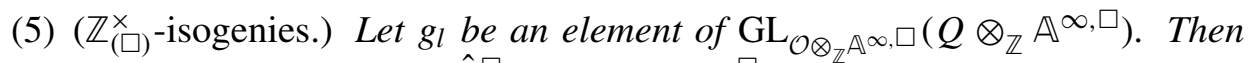
the submodule $g_{l}\left(Q \otimes_{\mathbb{Z}} \hat{\mathbb{Z}}^{\square}\right)$ in $Q \otimes_{\mathbb{Z}} A^{\infty, \square}$ determines a unique O-lattice $Q^{\prime}$ (up to isomorphism), together with a unique choice of an isomorphism $\left[g_{l}\right]_{Q}: Q \otimes_{\mathbb{Z}} \mathbb{Z}_{(\square)} \stackrel{\sim}{\rightarrow} Q^{\prime} \otimes_{\mathbb{Z}} \mathbb{Z}_{(\square)}$, inducing an isomorphism $Q \otimes_{\mathbb{Z}} \mathbb{A}^{\infty, \square} \stackrel{\sim}{\longrightarrow}$ $Q^{\prime} \otimes_{\mathbb{Z}} A^{\infty, \square}$ matching $g_{l}\left(Q \otimes_{\mathbb{Z}} \hat{\mathbb{Z}}^{\square}\right)$ with $Q^{\prime} \otimes_{\mathbb{Z}} \hat{\mathbb{Z}}^{\square}$, and inducing a canonical $\mathbb{Z}_{(\square)}^{\times}-i$ sogeny

$$
\left[g_{l}\right]_{Q}^{*}: \underline{\operatorname{Hom}}_{\mathcal{O}}\left(Q^{\prime}, G_{\mathrm{M}_{\mathcal{H}}}\right)^{\circ} \rightarrow \underline{\operatorname{Hom}}_{\mathcal{O}}\left(Q, G_{\mathrm{M}_{\mathcal{H}}}\right)^{\circ}
$$


defined by $\left[g_{l}\right]_{Q}$. For $\underline{\operatorname{Hom}}_{\mathcal{O}}\left(Q^{\prime}, G_{\mathrm{M}_{\mathcal{H}}}\right)^{\circ}$, there is also a directed partially ordered set $\mathbf{K}_{Q^{\prime}, \mathcal{H}, \Sigma}$ parametrizing (for $\left.\kappa^{\prime} \in \mathbf{K}_{Q^{\prime}, \mathcal{H}, \Sigma}\right) \mathbb{Z}_{(\square)}^{\times}$-isogenies

$$
\underline{\operatorname{Hom}}_{\mathcal{O}}\left(Q^{\prime}, G_{\mathrm{M}_{\mathcal{H}}}\right)^{\circ} \rightarrow \mathrm{N}_{\kappa^{\prime}}^{\prime}
$$

over $\mathrm{M}_{\mathcal{H}}$, together with open immersions $\mathrm{N}_{\kappa^{\prime}}^{\prime} \hookrightarrow\left(\mathrm{N}_{\kappa^{\prime}}^{\prime}\right)^{\text {tor }}$ of schemes over $\mathrm{S}_{0}$, satisfying analogues of properties (1)-(3) above. The constructions of $\mathbf{K}_{Q, \mathcal{H}, \Sigma}$ and $\mathbf{K}_{Q^{\prime}, \mathcal{H}, \Sigma}$ (and the objects they parametrize) satisfy the compatibility with $g_{l}$ in the sense that, for each $\kappa \in \mathbf{K}_{Q, \mathcal{H}, \Sigma}$, there is an element $\kappa^{\prime} \in \mathbf{K}_{Q^{\prime}, \mathcal{H}, \Sigma}$ such that the following are true:

(5a) The $\mathbb{Z}_{(\square)}^{\times}$-isogeny $\left[g_{l}\right]_{\kappa^{\prime}, \kappa}^{*}:=\kappa^{\text {isog }} \circ\left[g_{l}\right]_{Q}^{*} \circ\left(\left(\kappa^{\prime}\right)^{\text {isog }}\right)^{-1}: \mathrm{N}_{\kappa^{\prime}}^{\prime} \rightarrow \mathrm{N}_{\kappa}$ is an isogeny (not just a quasiisogeny), and hence defines a finite étale morphism.

(5b) There exists a (necessarily unique) proper log étale morphism

$$
\left(\left[g_{l}\right]_{\kappa^{\prime}, \kappa}^{*}\right)^{\text {tor }}:\left(\mathrm{N}_{\kappa^{\prime}}^{\prime}\right)^{\text {tor }} \rightarrow \mathrm{N}_{\kappa}^{\text {tor }}
$$

extending the morphism $\left[g_{l}\right]_{\kappa^{\prime}, \kappa}^{*}$ over $\mathrm{M}_{\mathcal{H}}$, such that

$$
\left.R^{i}\left(\left[g_{l}\right]_{\kappa^{\prime}, \kappa}^{*}\right)_{*}^{\text {tor }} \mathcal{O}_{\left(\mathrm{N}_{\kappa^{\prime}}^{\prime}\right.}\right)^{\text {tor }}=0
$$

for any $i>0$.

(5c) For any integer $i \geq 0$, there is a canonical isomorphism

$$
\left(\left(\left[g_{l}\right]_{\kappa^{\prime}, \kappa}^{*}\right)^{\text {tor }}\right)^{*}: \underline{H}_{\log -\mathrm{dR}}^{i}\left(\mathrm{~N}_{\kappa}^{\text {tor }} / \mathrm{M}_{\mathcal{H}, \Sigma}^{\text {tor }}\right) \stackrel{\sim}{\longrightarrow} \underline{H}_{\log -\mathrm{dR}}^{i}\left(\left(\mathrm{~N}_{\kappa^{\prime}}^{\prime}\right)^{\text {tor }} / \mathrm{M}_{\mathcal{H}, \Sigma}^{\text {tor }}\right)
$$

extending the canonical isomorphism

$$
\left(\left[g_{l}\right]_{\kappa^{\prime}, \kappa}^{*}\right)^{*}: \underline{H}_{\mathrm{dR}}^{i}\left(\mathrm{~N}_{\kappa} / \mathrm{M}_{\mathcal{H}}\right) \stackrel{\sim}{\longrightarrow} \underline{H}_{\mathrm{dR}}^{i}\left(\mathrm{~N}_{\kappa^{\prime}}^{\prime} / \mathrm{M}_{\mathcal{H}}\right)
$$

induced by $\left[g_{l}\right]_{Q}$, respecting the Hodge filtrations and inducing canonical isomorphisms

$$
\left.\left(\left(\left[g_{l}\right]_{\kappa^{\prime}, \kappa}^{*}\right)^{\text {tor }}\right)^{*}: R^{b} f_{*}^{\text {tor }}\left(\bar{\Omega}_{\mathrm{N}_{\kappa}^{\text {tor }} / \mathrm{M}_{\mathcal{H}}^{\text {tor }}}^{a}\right) \stackrel{\sim}{\longrightarrow} R^{b} f_{*}^{\text {tor }}\left(\bar{\Omega}_{\left(\mathrm{N}_{\kappa^{\prime}}^{\prime}\right.}^{a}\right)^{\text {tor }} / \mathrm{M}_{\mathcal{H}}^{\text {tor }}\right)
$$

(for integers $a+b=i$ ) compatible (under the canonical isomorphisms in (3) for $\mathrm{N}_{\kappa}^{\text {tor }}$ and $\left.\left(\mathrm{N}_{\kappa^{\prime}}^{\prime}\right)^{\text {tor }}\right)$ with the canonical isomorphisms

$$
\left(\left[g_{l}\right]_{Q}^{*}\right)^{*}: \underline{\operatorname{Hom}}_{\mathcal{O}}\left(Q^{\vee}, \underline{\operatorname{Lie}}_{G^{\vee} / \mathrm{M}_{\mathcal{H}}^{\text {tor }}}\right) \stackrel{\sim}{\longrightarrow} \underline{\operatorname{Hom}}_{\mathcal{O}}\left(\left(Q^{\prime}\right)^{\vee}, \underline{\operatorname{Lie}}_{G^{\vee}} / M_{\mathcal{H}}^{\text {tor }}\right)
$$

and

$$
\left(\left[g_{l}\right]_{Q}^{*}\right)^{*}: \underline{\operatorname{Hom}}_{\mathcal{O}}\left(Q^{\vee}, \underline{\operatorname{Lie}}_{G / \mathrm{M}_{\mathcal{H}}^{\text {tor }}}\right) \stackrel{\sim}{\longrightarrow} \underline{\operatorname{Hom}}_{\mathcal{O}}\left(\left(Q^{\prime}\right)^{\vee}, \underline{\operatorname{Lie}}_{G / \mathrm{M}_{\mathcal{H}}^{\text {tor }}}\right) .
$$


2C. Outline of the proof. The proof of Theorem 2.15 consists of seven steps:

(1) Find a PEL-type $\mathcal{O}$-lattice $(\widetilde{L},\langle\cdot, \cdot \tau, \tilde{h})$, a fully symplectic admissible filtration $\widetilde{Z}$ on $\widetilde{L} \otimes_{\mathbb{Z}} \hat{\mathbb{Z}}^{\square}$, a torus argument $\widetilde{\Phi}$, and a splitting $\tilde{\delta}$ for $\widetilde{Z}$, such that, for some choices of $\widetilde{\mathcal{H}}, \widetilde{\Sigma}$, and $\widetilde{\sigma}$, the $\left[\left(\widetilde{\Phi}_{\widetilde{\mathcal{H}}}, \tilde{\delta}_{\widetilde{\mathcal{H}}}, \widetilde{\sigma}\right)\right]$-stratum $\widetilde{Z}_{\left[\left(\widetilde{\Phi}_{\tilde{\mathcal{H}}}, \tilde{\delta}_{\tilde{\mathcal{H}}}, \widetilde{\sigma}\right)\right]}$ of the toroidal compactification $\widetilde{\mathrm{M}}_{\widetilde{\mathcal{H}}}^{\text {tor }}=\widetilde{\mathrm{M}}_{\widetilde{\mathcal{H}}, \widetilde{\Sigma}}^{\text {tor }} \widetilde{\mathcal{L}}$ has a canonical structure of an abelian scheme over $M_{\mathcal{H}}$, and such that there exists a canonical $\mathbb{Z}_{(\square)}^{\times}$-isogeny

$$
\kappa^{\text {isog }}: \underline{\operatorname{Hom}}_{\mathcal{O}}\left(Q, G_{\mathrm{M}_{\mathcal{H}}}\right)^{\circ} \rightarrow \widetilde{\mathrm{Z}}_{\left[\left(\widetilde{\Phi}_{\tilde{\mathcal{H}}}, \tilde{\delta}_{\tilde{\mathcal{H}}}, \widetilde{\sigma}\right)\right]} \cdot
$$

Then we take $\mathrm{N}_{\kappa}$ to be this $\widetilde{\mathrm{Z}}_{\left[\left(\widetilde{\Phi}_{\tilde{\mathcal{H}}}, \tilde{\delta}_{\tilde{\mathcal{H}}}, \widetilde{\sigma}\right)\right]}$.

Take $\mathbf{K}_{Q, \mathcal{H}, \Sigma}^{\text {pre }}$ to be the set of all such triples $\kappa=(\widetilde{\mathcal{H}}, \widetilde{\Sigma}, \widetilde{\sigma})$, with directed partial order defined by the relation

$$
\kappa^{\prime}=\left(\widetilde{\mathcal{H}}^{\prime}, \widetilde{\Sigma}^{\prime}, \widetilde{\sigma}^{\prime}\right) \succ \kappa=(\widetilde{\mathcal{H}}, \widetilde{\Sigma}, \widetilde{\sigma})
$$

when $\widetilde{\mathcal{H}}^{\prime} \subset \widetilde{\mathcal{H}}$ and $\widetilde{\Sigma}^{\prime}$ refines $\widetilde{\Sigma}$ as in [Lan 2008, Definition 6.4.2.8], and when the $\left[\left(\widetilde{\Phi}_{\widetilde{\mathcal{H}}^{\prime}}, \tilde{\delta}_{\widetilde{\mathcal{H}}^{\prime}}, \widetilde{\sigma}^{\prime}\right)\right]$-stratum of $\widetilde{\mathrm{M}}_{\widetilde{\mathcal{H}}^{\prime}, \widetilde{\Sigma}^{\prime}}^{\text {tor }}$ is mapped (surjectively) to the

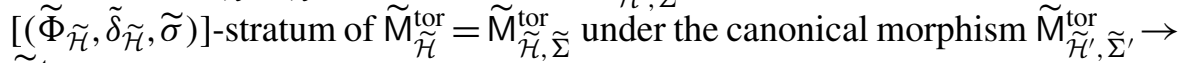
$\tilde{\mathrm{M}}_{\widetilde{\mathcal{H}}, \widetilde{\Sigma}}^{\text {tor }}$ given by [Lan 2008, Proposition 6.4.2.9].

For $\kappa=(\widetilde{\mathcal{H}}, \widetilde{\Sigma}, \widetilde{\sigma})$, take $\mathrm{N}_{\kappa}^{\text {tor }}$ to be the closure of the $\left[\left(\widetilde{\Phi}_{\widetilde{\mathcal{H}}}, \tilde{\delta}_{\tilde{\mathcal{H}}}, \widetilde{\sigma}\right)\right]$-stratum in

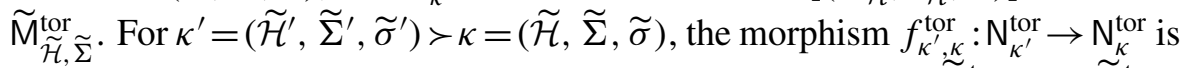
just the morphism induced by the canonical proper morphism $\tilde{\mathrm{M}}_{\widetilde{\mathcal{H}}^{\prime}, \widetilde{\Sigma}^{\prime}}^{\text {tor }} \rightarrow \widetilde{\mathrm{M}}_{\widetilde{\mathcal{H}}, \widetilde{\Sigma}}^{\text {tor }}$ given by [Lan 2008, Proposition 6.4.2.9].

(2) Show that $\mathrm{N}_{\kappa}^{\text {tor }}$ is projective and smooth over $\mathrm{S}_{0}$ for $\kappa \in \mathbf{K}_{Q, \mathcal{H}, \Sigma}^{\mathrm{pre}}$.

(3) Find a condition on $\kappa$ that guarantees the existence of a morphism $f_{\kappa}^{\text {tor }}: \mathrm{N}_{\kappa}^{\text {tor }} \rightarrow$ $\mathrm{M}_{\mathcal{H}}^{\text {tor }}$ extending the structural morphism $f_{\kappa}: \mathrm{N}_{\kappa} \rightarrow \mathrm{M}_{\mathcal{H}}$.

(4) Take $\mathbf{K}_{Q, \mathcal{H}, \Sigma}$ to be the subset of $\mathbf{K}_{Q, \mathcal{H}, \Sigma}^{\text {pre }}$ consisting of elements $\kappa$ satisfying the condition we have found. Show that this subset is nonempty and has an induced directed partial order by showing that the conditions we need can be achieved after suitable refinements of cone decompositions. This verifies (1) and (2) of Theorem 2.15.

(5) For each $\kappa \in \mathbf{K}_{Q, \mathcal{H}, \Sigma}$, verify that the morphism $f_{\kappa}^{\text {tor }}: \mathrm{N}_{\kappa}^{\text {tor }} \rightarrow \mathrm{M}_{\mathcal{H}}^{\text {tor }}$ extending $\mathrm{N}_{\kappa} \rightarrow \mathrm{M}_{\mathcal{H}}$ is $\log$ smooth, and verify (3a) of Theorem 2.15.

(6) Assuming (3b) and (3c), verify (4) and (5) of Theorem 2.15 using the Hecke actions on the double tower $\left\{\widetilde{\mathrm{M}}_{\widetilde{\mathcal{H}}}, \widetilde{\Sigma}\right\}_{\tilde{\mathcal{H}}}, \widetilde{\Sigma}$.

(7) Verify (3b), (3c), and (3d) of Theorem 2.15 using explicit descriptions of the formal fibers of $f_{\kappa}^{\text {tor }}$ along (locally closed) strata of $\mathrm{M}_{\mathcal{H}}^{\text {tor }}$. (A crucial step for (3b) requires the notion of log extensions of polarizations we mentioned in the introduction.) 
We will carry out these steps in Sections 3-5. We will make frequent references to results cited in Section 1, and also to the original statements in [Lan 2008].

2D. System of notation. Although the underlying ideas are simple, the notation can be quite heavy. (This seems unavoidable in general works on compactifications.) We decided to keep the notation informative (and hence complicated), because we believe it is more difficult to keep track of three sets of cusp labels and cone decompositions with simplified notation. We understand that the heaviness of notation will inevitably be an enormous burden on the readers, and hence we would like to provide some guidance by explaining the key features in the system of notation, as follows:

- The superscript ${ }^{\text {tor }}$ stands for toroidal compactifications (or objects related to them). For morphisms this typically means extensions to morphisms between toroidal compactifications.

- Depending on the context, the overlines can have different meanings:

- For geometric objects they almost always mean closures.

- For sheaves of differentials (or related objects) they mean the log versions.

- Notable exceptions (to the above two) are in Sections 3B-3C below, where overlines can also stand for quotients of group schemes or sheaves.

- Objects for the "given" moduli problem $\mathrm{M}_{\mathcal{H}}$ and its compactifications are denoted as in Section 1.

- Objects for the "larger" moduli problem $\widetilde{\mathrm{M}}_{\widetilde{\mathcal{H}}}$ (mentioned in step (1) above) will be denoted with either ${ }^{\sim}$ (tilde) or ${ }^{\sim}$ (breve) on top of the symbols in Section 1. The difference is the following:

- Symbols with ${ }^{\sim}$ will be used for defining $\tilde{M}_{\widetilde{\mathcal{H}}}$ and its compactifications $\widetilde{M}_{\widetilde{\mathcal{H}}, \widetilde{\Sigma}}^{\text {tor }}$, and for realizing the Kuga families we would like to compactify as

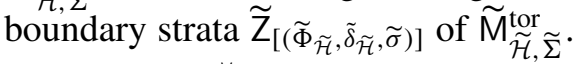

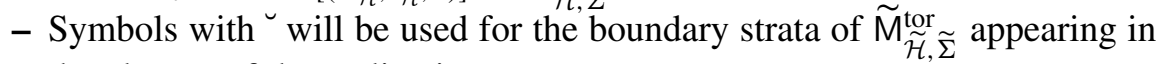
the closure of the realizations

$$
\widetilde{Z}_{\left[\left(\widetilde{\Phi}_{\tilde{\mathcal{H}}}, \tilde{\delta}_{\tilde{\mathcal{H}}}, \widetilde{\sigma}\right)\right]} \cdot
$$

(These strata are parametrized by faces $\left[\left(\breve{\Phi}_{\widetilde{\mathcal{H}}}, \breve{\delta}_{\widetilde{\mathcal{H}}}, \breve{\tau}\right)\right]$ of $\left[\left(\widetilde{\Phi}_{\widetilde{\mathcal{H}}}, \tilde{\delta}_{\widetilde{\mathcal{H}}}, \widetilde{\sigma}\right)\right]$.) In other words, they parametrize the boundary strata of the toroidal compactification of the Kuga families we consider.

- In the local descriptions of toroidal boundary structures, we will encounter notations of the forms $(\cdot)(\sigma)$ and $(\cdot)_{\sigma}$.

- When the object $(\cdot)$ being modified is a scheme with action by some torus, $(\cdot)(\sigma)$ will stand for the affine toroidal embedding adding the $\sigma$-stratum 
(which then also adds all the strata for nontrivial faces of $\sigma$ ), while $(\cdot)_{\sigma}$ will stand for the closed $\sigma$-stratum (without the nontrivial face strata).

- The formal version of $(\cdot)_{\sigma}$ (often denoted in Fraktur) will mean the formal completion of $(\cdot)(\sigma)$ along $(\cdot)_{\sigma}$.

The notation will be most heavy in Sections 4-5, where the calculation of relative cohomology is carried out in detail. For readers only interested in applications to cohomology of Shimura varieties, the statements of Theorem 2.15, the two propositions in Section 3D, and the applications in Section 6 are all they need.

\section{Constructions of compactifications and morphisms}

3A. Kuga families as toroidal boundary strata. The goal of this subsection is to carry out steps (1) and (2) of Section 2C.

Let $Q$ be an $\mathcal{O}$-lattice as in Theorem 2.15. Identify $Q^{\vee}$ with $\operatorname{Hom}_{\mathcal{O}}\left(Q, \operatorname{Diff}^{-1}\right)$ and give it an $\mathcal{O}$-lattice structure as in Section 2A. The (surjective) trace map $\operatorname{Tr}_{\mathcal{O} / \mathbb{Z}}:$ Diff $^{-1} \rightarrow \mathbb{Z}$ induces a perfect pairing

$$
\langle\cdot, \cdot\rangle_{Q}: Q^{\vee} \times Q \rightarrow \mathbb{Z}:(f, x) \mapsto \operatorname{Tr}_{\mathcal{O} / \mathbb{Z}}(f(x)) .
$$

By extension of scalars, the pairing $\langle\cdot, \cdot\rangle_{Q}$ induces a perfect pairing between $Q^{\vee} \otimes_{\mathbb{Z}} \mathbb{Q}$ and $Q \otimes_{\mathbb{Z}} \mathbb{Q}$. By Condition 1.9, the action of $\mathcal{O}$ on $L$ extends to an action of some maximal order $\mathcal{O}^{\prime}$ in $B$ containing $\mathcal{O}$. Let us fix the choice of such a maximal order $\mathcal{O}^{\prime}$. By [Lan 2008, Proposition 1.1.1.17], $\mathcal{O} \otimes_{\mathbb{Z}} \mathbb{Z}_{(p)} \neq \mathcal{O}^{\prime} \otimes_{\mathbb{Z}} \mathbb{Z}_{(p)}$ for a prime number $p>0$ only when $p \mid$ Disc. Let $Q_{0}:=\mathcal{O}^{\prime} \cdot Q \subset Q \otimes_{\mathbb{Z}} \mathbb{Q}$ and $Q_{-2}:=\operatorname{Hom}_{\mathcal{O}}\left(Q, \operatorname{Diff}_{\mathcal{O}^{\prime} / \mathbb{Z}}^{-1}\right)(1) \subset Q^{\vee} \otimes_{\mathbb{Z}} \mathbb{Q}(1)$. Then the induced pairing

$$
\langle\cdot, \cdot\rangle_{Q}: Q_{-2} \times Q_{0} \rightarrow \mathbb{Q}(1)
$$

has values in $\mathbb{Z}(1)$. The localizations of this pairing at primes of $\mathbb{Z}$ are perfect except at those dividing Disc.

Let $(\tilde{L},\langle\cdot, \cdot \gamma, \tilde{h})$ be the symplectic $\mathcal{O}$-lattice given by the following data:

(1) An $\mathcal{O}$-lattice $\widetilde{L}:=Q_{-2} \oplus L \oplus Q_{0}$, where $Q_{-2}$ and $Q_{0}$ are defined as above. (Note that $\widetilde{L}$ satisfies Condition 1.9 by construction.)

(2) A symplectic $\mathcal{O}$-pairing $\langle\cdot, \cdot\rangle^{\sim}: \widetilde{L} \times \widetilde{L} \rightarrow \mathbb{Z}(1)$ defined (symbolically) by the matrix

$$
\langle x, y\rangle^{\sim}:={ }^{t}\left(\begin{array}{c}
x_{-2} \\
x_{-1} \\
x_{0}
\end{array}\right)\left(\begin{array}{cc}
\langle\cdot, \cdot\rangle_{Q} \\
-{ }^{t}\langle\cdot, \cdot\rangle_{Q} & \langle\cdot, \cdot\rangle
\end{array}\right)\left(\begin{array}{c}
y_{-2} \\
y_{-1} \\
y_{0}
\end{array}\right),
$$

namely by

$$
\langle x, y\rangle^{\sim}:=\left\langle x_{-2}, y_{0}\right\rangle_{Q}+\left\langle x_{-1}, y_{-1}\right\rangle-\left\langle y_{-2}, x_{0}\right\rangle_{Q},
$$


where $x=\left(\begin{array}{c}x_{-2} \\ x_{-1} \\ x_{0}\end{array}\right)$ and $y=\left(\begin{array}{c}y_{-2} \\ y_{-1} \\ y_{0}\end{array}\right)$ are elements of $\tilde{L}=Q_{-2} \oplus L \oplus Q_{0}$ expressed (vertically) in terms of components in the direct summands.

Let $j_{Q}: Q^{\vee} \hookrightarrow Q$ be an embedding of $\mathcal{O}$-lattices given by Lemma 2.5 , so that the pairing $\left\langle j_{Q}^{-1}(\cdot), \cdot\right\rangle_{Q}:\left(Q \otimes_{\mathbb{Z}} \mathbb{R}\right) \times\left(Q \otimes_{\mathbb{Z}} \mathbb{R}\right) \rightarrow \mathbb{R}$ is positive definite. Consider the $\mathbb{R}$-algebra homomorphism $\tilde{h}: \mathbb{C} \rightarrow \operatorname{End}_{\mathcal{O} \otimes_{\mathbb{Z}} \mathbb{R}}\left(\tilde{L} \otimes_{\mathbb{Z}} \mathbb{R}\right)$ defined by

$$
\begin{aligned}
z & =z_{1}+\sqrt{-1} z_{2} \\
& \mapsto \tilde{h}(z):=\left(\begin{array}{ccc}
z_{1} \operatorname{Id}_{Q_{-2} \otimes_{\mathbb{Z}} \mathbb{R}} & -z_{2}\left((2 \pi \sqrt{-1}) \circ j_{Q}^{-1}\right) \\
z_{2}\left(j_{Q} \circ(2 \pi \sqrt{-1})^{-1}\right) & h(z) & z_{1} \operatorname{Id}_{Q_{0} \otimes_{\mathbb{Z}} \mathbb{R}}
\end{array}\right),
\end{aligned}
$$

where $2 \pi \sqrt{-1}: \mathbb{Z} \stackrel{\sim}{\longrightarrow} \mathbb{Z}(1)$ and $(2 \pi \sqrt{-1})^{-1}: \mathbb{Z}(1) \stackrel{\sim}{\longrightarrow} \mathbb{Z}$ stand for the isomorphisms defined by the choice of $\sqrt{-1}$ in $\mathbb{C}$, and where the matrix acts (symbolically) on elements of $\widetilde{L} \otimes_{\mathbb{Z}} \mathbb{R}$ by left multiplication. In other words,

$$
\tilde{h}(z)\left(\begin{array}{c}
x_{-2} \\
x_{-1} \\
x_{0}
\end{array}\right)=\left(\begin{array}{c}
z_{1} x_{-2}-z_{2}\left((2 \pi \sqrt{-1}) \circ j_{Q}^{-1}\right)\left(x_{0}\right) \\
h(z) x_{-1} \\
z_{2}\left(j_{Q} \circ(2 \pi \sqrt{-1})^{-1}\right)\left(x_{-2}\right)+z_{1} x_{0}
\end{array}\right) .
$$

Then $\tilde{h}$ is a polarization of $(\tilde{L},\langle\cdot, \cdot \gamma)$ making $(\tilde{L},\langle\cdot, \cdot \gamma, \tilde{h})$ a PEL-type $\mathcal{O}$-lattice. Note that the reflex field of $\left(\widetilde{L} \otimes_{\mathbb{Z}} \mathbb{R},\langle\cdot, \cdot \gamma, \tilde{h})\right.$ is also $F_{0}$.

By construction of $(\widetilde{L},\langle\cdot, \cdot \gamma)$, there is a fully symplectic admissible filtration on $\widetilde{L} \otimes_{\mathbb{Z}} \hat{\mathbb{Z}}^{\square}$ induced by

$$
0 \subset Q_{-2} \subset Q_{-2} \oplus L \subset Q_{-2} \oplus L \oplus Q_{0}=\widetilde{L} .
$$

More precisely, we have

$$
\begin{aligned}
\widetilde{\mathrm{Z}}_{-3} & :=0, \\
\widetilde{\mathrm{Z}}_{-2} & :=Q_{-2} \otimes_{\mathbb{Z}} \hat{\mathbb{Z}}^{\square}, \\
\widetilde{\mathrm{Z}}_{-1} & :=\left(Q_{-2} \otimes_{\mathbb{Z}} \hat{\mathbb{Z}}^{\square}\right) \oplus\left(L \otimes_{\mathbb{Z}} \hat{\mathbb{Z}}^{\square}\right), \\
\widetilde{\mathrm{Z}}_{0} & :=\left(Q_{-2} \otimes_{\mathbb{Z}} \hat{\mathbb{Z}}^{\square}\right) \oplus\left(L \otimes_{\mathbb{Z}} \hat{\mathbb{Z}}^{\square}\right) \oplus\left(Q_{0} \otimes_{\mathbb{Z}} \hat{\mathbb{Z}}^{\square}\right)=\widetilde{L} \otimes_{\mathbb{Z}} \hat{\mathbb{Z}}^{\square},
\end{aligned}
$$

so that there are canonical isomorphisms

$$
\mathrm{Gr}_{-2}^{\widetilde{z}} \cong Q_{-2} \otimes_{\mathbb{Z}} \hat{\mathbb{Z}}^{\square}, \quad \mathrm{Gr}_{-1}^{\widetilde{\mathrm{z}}} \cong L \otimes_{\mathbb{Z}} \hat{\mathbb{Z}}^{\square}, \quad \mathrm{Gr}_{0}^{\widetilde{\mathrm{z}}} \cong Q_{0} \otimes_{\mathbb{Z}} \hat{\mathbb{Z}}^{\square}
$$

matching the pairings $\mathrm{Gr}_{-2}^{\widetilde{\mathrm{z}}} \times \mathrm{Gr}_{0}^{\widetilde{\mathrm{z}}} \rightarrow \hat{\mathbb{Z}}^{\square}(1)$ and $\mathrm{Gr}_{-1}^{\widetilde{\mathrm{z}}} \times \mathrm{Gr}_{-1}^{\widetilde{\mathrm{z}}} \rightarrow \hat{\mathbb{Z}}^{\square}(1)$ induced by $\langle\cdot, \cdot\rangle^{\sim}$ with $\langle\cdot, \cdot\rangle_{Q}$ and $\langle\cdot, \cdot\rangle$, respectively. 
Let $\tilde{X}:=\operatorname{Hom}_{\mathcal{O}}\left(Q_{-2}, \operatorname{Diff}^{-1}(1)\right)$ and $\tilde{Y}:=Q_{0}$. The pairing

$$
\langle\cdot, \cdot\rangle_{Q}: Q_{-2} \times Q_{0} \rightarrow \mathbb{Z}(1)
$$

induces a canonical embedding $\tilde{\phi}: \widetilde{Y} \hookrightarrow \widetilde{X}$ and there are canonical isomorphisms $\widetilde{\varphi}_{-2}: \mathrm{Gr}_{-2}^{\widetilde{Z}} \stackrel{\sim}{\rightarrow} \operatorname{Hom}_{\hat{\mathbb{Z}}}\left(\widetilde{X} \otimes_{\mathbb{Z}} \hat{\mathbb{Z}}^{\square}, \hat{\mathbb{Z}}^{\square}(1)\right)$ and $\widetilde{\varphi}_{0}: \mathrm{Gr}_{0}^{\widetilde{Z}} \stackrel{\sim}{\rightarrow} \widetilde{Y} \otimes_{\mathbb{Z}} \hat{\mathbb{Z}}^{\square}$ (of $\hat{\mathbb{Z}}^{\square}$-modules). These data define a torus argument $\widetilde{\Phi}:=\left(\widetilde{X}, \widetilde{Y}, \widetilde{\phi}, \widetilde{\varphi}-2, \widetilde{\varphi}_{0}\right)$ for $\widetilde{Z}$ as in Definition 1.13.

Let $\tilde{\delta}$ be the obvious splitting of $\widetilde{Z}$ induced by the equality $Q_{-2} \oplus L \oplus Q_{0}=\widetilde{L}$.

Let $\widetilde{\mathrm{G}}$ be the group functor defined by $(\widetilde{L},\langle\cdot, \cdot \gamma)$ as in Definition 1.1. For any $\hat{\mathbb{Z}}^{\square}$-algebra $R$, let $\widetilde{\mathrm{P}}_{\widetilde{\mathrm{Z}}}(R)$ denote the subgroup of $\widetilde{\mathrm{G}}(R)$ consisting of elements $g$ such that $g\left(\widetilde{Z}_{-2} \otimes_{\hat{\mathbb{Z}} \square} R\right)=\widetilde{\mathrm{Z}}_{-2} \otimes_{\hat{\mathbb{Z}} \square} R$ and $g\left(\widetilde{\mathrm{Z}}_{-1} \otimes_{\hat{\mathbb{Z}} \square} R\right)=\widetilde{\mathrm{Z}}_{-1} \otimes_{\hat{\mathbb{Z}} \square} R$. Any element $g$ in $\widetilde{\mathrm{P}}_{\widetilde{\mathrm{Z}}}(R)$ defines an isomorphism

$$
\mathrm{Gr}_{-1}^{\widetilde{Z}}(g): \mathrm{Gr}_{-1}^{\widetilde{Z}} \otimes_{\hat{\mathbb{Z}} \square} R \stackrel{\sim}{\longrightarrow} \mathrm{Gr}_{-1}^{\widetilde{z}} \otimes_{\hat{\mathbb{Z}} \square} R,
$$

which corresponds under the canonical isomorphism $\mathrm{Gr}_{-1}^{\tilde{\mathrm{z}}} \otimes_{\hat{\mathbb{Z}} \square} R \cong L \otimes_{\mathbb{Z}} R$ above to an element of $\mathrm{G}(R)$. This defines in particular a homomorphism

$$
\mathrm{Gr}_{-1}^{\widetilde{Z}}: \widetilde{\mathrm{P}}_{\widetilde{\mathrm{Z}}}\left(\hat{\mathbb{Z}}^{\square}\right) \rightarrow \mathrm{G}\left(\hat{\mathbb{Z}}^{\square}\right) \text {. }
$$

Let $\widetilde{\mathcal{H}}$ be any neat open compact subgroup of $\widetilde{\mathrm{G}}\left(\hat{\mathbb{Z}}^{\square}\right)$ such that the image $\operatorname{Gr}_{-1}^{\widetilde{z}}\left(\widetilde{\mathcal{H}} \cap \widetilde{\mathrm{P}}_{\widetilde{Z}}\left(\hat{\mathbb{Z}}^{\square}\right)\right)$ is exactly $\mathcal{H}$. (Such an $\widetilde{\mathcal{H}}$ exists because the pairing $\left\langle\cdot, \cdot \gamma^{\sim}\right.$ is the direct sum of the pairings on $Q_{-2} \oplus Q_{0}$ and on $L$.) The data of $\mathcal{O},(\widetilde{L},\langle\cdot, \cdot \gamma, \tilde{h})$, $\square$, and $\widetilde{\mathcal{H}} \subset \widetilde{\mathrm{G}}\left(\hat{\mathbb{Z}}^{\square}\right)$ define a moduli problem $\widetilde{\mathrm{M}}_{\tilde{\mathcal{H}}}$ as in Definition 1.6.

Take any compatible choice $\widetilde{\Sigma}$ of admissible smooth rational polyhedral cone decomposition data for $\widetilde{\mathrm{M}}_{\widetilde{\mathcal{H}}}$ that is projective (see Definitions 1.33 and 1.34). Since $\widetilde{\mathcal{H}}$ is neat, any such $\widetilde{\Sigma}$ defines a toroidal compactification $\widetilde{M}_{\widetilde{\mathcal{H}}}^{\text {tor }}=\widetilde{M}_{\widetilde{\mathcal{H}}}^{\text {tor }}, \widetilde{\Sigma}$ which is projective and smooth over $\mathrm{S}_{0}$ by (7) of Theorem 1.41 .

Let $(\widetilde{Z}, \widetilde{\Phi}, \tilde{\delta})$ be as above, and let $\left(\widetilde{\mathrm{Z}}_{\widetilde{\mathcal{H}}}, \widetilde{\Phi}_{\widetilde{\mathcal{H}}}=\left(\widetilde{X}, \widetilde{Y}, \widetilde{\phi}, \widetilde{\varphi}_{-2, \widetilde{\mathcal{H}}}, \widetilde{\varphi}_{0, \widetilde{\mathcal{H}}}\right), \tilde{\delta}_{\widetilde{\mathcal{H}}}\right)$ be the induced triple at level $\widetilde{\mathcal{H}}$, inducing a cusp label $\left.\left[\widetilde{\mathrm{Z}}_{\widetilde{\mathcal{H}}}, \widetilde{\Phi}_{\widetilde{\mathcal{H}}}, \tilde{\delta}_{\widetilde{\mathcal{H}}}\right)\right]$ at level $\widetilde{\mathcal{H}}$.

Let $\widetilde{\sigma} \subset \mathbf{P}_{\widetilde{\Phi}}^{+}$be any top-dimensional nondegenerate rational polyhedral cone in the cone decomposition $\widetilde{\Sigma}_{\widetilde{\Phi}_{\widetilde{\mathcal{H}}}}$ in $\widetilde{\Sigma}$. Then, by (2) of Theorem 1.41, we have a

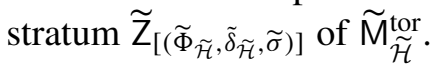

Since $\widetilde{\sigma}$ is a top-dimensional cone in $\widetilde{\Sigma}_{\widetilde{\Phi}_{\tilde{\mathcal{H}}}}$, the locally closed stratum $\widetilde{Z}_{\left[\left(\widetilde{\Phi}_{\tilde{\mathcal{H}}}, \tilde{\delta}_{\tilde{\mathcal{H}}}, \widetilde{\sigma}\right)\right]}$ (not its closure) is a zero-dimensional torus bundle over the abelian scheme $\widetilde{\widetilde{C}}_{\widetilde{\Phi}} \widetilde{\widetilde{Q}}_{\tilde{\mathcal{H}}}, \tilde{\delta}_{\tilde{\mathcal{H}}}$

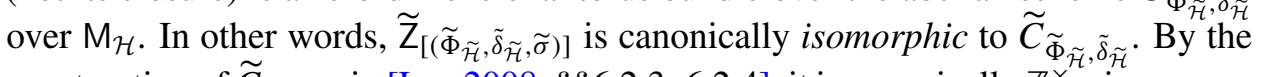
construction of $\widetilde{C}_{\widetilde{\Phi}_{\tilde{\mathcal{H}}}, \tilde{\delta}_{\tilde{\mathcal{H}}}}$ in [Lan 2008, $\left.\S \S 6.2 .3-6.2 .4\right]$, it is canonically $\mathbb{Z}_{(\square)}^{\times}$-isogenous to the abelian scheme $\underline{\operatorname{Hom}}_{\mathcal{O}}\left(Q, G_{\mathrm{M}_{\mathcal{H}}}\right)^{\circ}$. Let us define $\mathrm{N}_{\kappa}$ to be this stratum

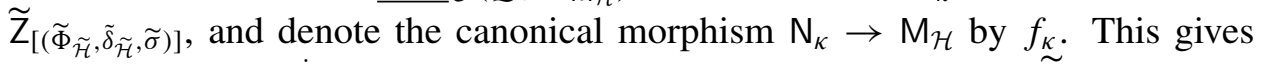
the $\mathbb{Z}_{(\square)}^{\times}$-isogeny $\kappa^{\text {isog }}: \underline{\operatorname{Hom}}_{\widetilde{C}_{\mathcal{O}}}\left(Q, G_{\mathrm{M}_{\mathcal{H}}}\right)^{\circ} \rightarrow \mathrm{N}_{\kappa}$. Note that $\mathrm{N}_{\kappa}=\widetilde{\mathrm{Z}}_{\left[\left(\widetilde{\Phi}_{\tilde{\mathcal{H}}}, \tilde{\delta}_{\tilde{\mathcal{H}}}, \widetilde{\sigma}_{\tilde{\sigma}}\right)\right]}$ is canonically isomorphic to $\widetilde{C}_{\widetilde{\Phi}_{\tilde{\mathcal{H}}}, \tilde{\delta}_{\widetilde{\mathcal{H}}}}$ for every $\widetilde{\Sigma}$ and every top-dimensional cone $\widetilde{\sigma}$ in $\widetilde{\Sigma}_{\widetilde{\Phi}_{\tilde{\mathcal{H}}}}$. 
As planned in step (1) of Section $2 \mathrm{C}$, let us take $\mathbf{K}_{Q, \mathcal{H}, \Sigma}^{\mathrm{pre}}$ to be the set of all possible such triples $\kappa=(\widetilde{\mathcal{H}}, \widetilde{\Sigma}, \widetilde{\sigma})$, with directed partial order defined by the relation $\kappa^{\prime}=\left(\widetilde{\mathcal{H}}^{\prime}, \widetilde{\Sigma}^{\prime}, \widetilde{\sigma}^{\prime}\right) \succ \kappa=(\widetilde{\mathcal{H}}, \widetilde{\Sigma}, \widetilde{\sigma})$ when $\widetilde{\mathcal{H}}^{\prime} \subset \widetilde{\mathcal{H}}$, when $\widetilde{\Sigma}^{\prime}$ refines $\widetilde{\Sigma}$ as in [Lan 2008, Definition 6.4.2.8], and when $\left(\widetilde{\Phi}_{\widetilde{\mathcal{H}}^{\prime}}, \tilde{\delta}_{\tilde{\mathcal{H}}^{\prime}}, \widetilde{\sigma}^{\prime}\right)$ refines $\left(\widetilde{\Phi}_{\tilde{\mathcal{H}}}, \tilde{\delta}_{\tilde{\mathcal{H}}}, \widetilde{\sigma}\right)$ as in [Lan 2008, Definition 6.4.2.6]. In this case, the $\left[\left(\widetilde{\Phi}_{\widetilde{\mathcal{H}}^{\prime}}, \tilde{\delta}_{\widetilde{\mathcal{H}}^{\prime}}, \widetilde{\sigma}^{\prime}\right)\right]$-stratum of $\widetilde{M}_{\widetilde{\mathcal{H}}^{\prime}, \widetilde{\Sigma}^{\prime}}^{\text {tor }}$ is mapped to the $\left[\left(\widetilde{\Phi}_{\widetilde{\mathcal{H}}}, \tilde{\delta}_{\widetilde{\mathcal{H}}}, \widetilde{\sigma}\right)\right]$-stratum of $\widetilde{\mathrm{M}}_{\widetilde{\mathcal{H}}, \widetilde{\Sigma}}^{\text {tor }}, \widetilde{\mathcal{L}}^{\text {to }}$ by the canonical morphism $\widetilde{\bar{M}}_{\widetilde{\mathcal{H}}^{\prime}, \widetilde{\Sigma}^{\prime}}^{\widetilde{\mathcal{H}}^{\prime}, \widetilde{\Sigma}^{\prime}} \rightarrow \widetilde{\mathrm{M}}_{\widetilde{\mathcal{H}}, \widetilde{\Sigma}}^{\text {tor }}$ given by [Lan 2008, Proposition 6.4.2.9]. Note that the induced morphism $f_{\kappa^{\prime}, \kappa}: \mathrm{N}_{\kappa^{\prime}} \rightarrow \mathrm{N}_{\kappa}$, which is $\kappa^{\text {isog }} \circ\left(\left(\kappa^{\prime}\right)^{\text {isog }}\right)^{-1}$ by definition, can be

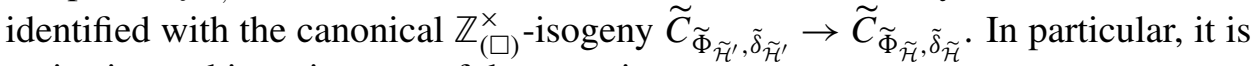
surjective and is an isogeny of degree prime to $\square$.

For $\kappa=(\widetilde{\mathcal{H}}, \widetilde{\Sigma}, \widetilde{\sigma})$, take $\mathrm{N}_{\kappa}^{\text {tor }}$ to be the closure of $\widetilde{Z}_{\left[\left(\widetilde{\Phi}_{\tilde{\mathcal{H}}}, \tilde{\delta}_{\tilde{\mathcal{H}}}, \widetilde{\sigma}\right)\right]}$ in $\widetilde{\mathrm{M}}_{\widetilde{\mathcal{H}}, \widetilde{\Sigma}}^{\text {tor }}$. Then we obtain the canonical immersion $\kappa^{\text {tor }}: \mathrm{N}_{\kappa} \hookrightarrow \mathrm{N}_{\kappa}^{\text {tor }}$.

When $\kappa^{\prime}=\left(\widetilde{\mathcal{H}}^{\prime}, \widetilde{\Sigma}^{\prime}, \widetilde{\sigma}^{\prime}\right) \succ \kappa=(\widetilde{\mathcal{H}}, \widetilde{\Sigma}, \widetilde{\sigma})$, the morphism $f_{\kappa^{\prime}, \kappa}^{\text {tor }}: \mathrm{N}_{\kappa^{\prime}}^{\text {tor }} \rightarrow \mathrm{N}_{\kappa}^{\text {tor }}$ is simply the morphism induced by the canonical proper morphism $\widetilde{M}_{\widetilde{\mathcal{H}}^{\prime}, \widetilde{\Sigma}^{\prime}}^{\text {tor }} \rightarrow \widetilde{\mathrm{M}}_{\widetilde{\mathcal{H}}, \widetilde{\Sigma}}^{\text {tor }}$ given by [Lan 2008, Proposition 6.4.2.9]. Note that the latter morphism is étale locally given by equivariant morphisms between toric schemes, and the same is true for the induced morphism $f_{\kappa^{\prime}, \kappa}^{\text {tor }}: \mathrm{N}_{\kappa^{\prime}}^{\text {tor }} \rightarrow \mathrm{N}_{\kappa}^{\text {tor }}$. Therefore, both the morphism $\tilde{\mathrm{M}}_{\widetilde{\mathcal{H}}^{\prime}, \widetilde{\Sigma}^{\prime}}^{\text {tor }} \rightarrow \widetilde{\mathrm{M}}_{\widetilde{\mathcal{H}}, \widetilde{\Sigma}}^{\text {tor }}, \widetilde{\Sigma}^{\text {and }}$ and the induced morphism $f_{\kappa^{\prime}, \kappa}^{\text {tor }}: \mathrm{N}_{\kappa^{\prime}}^{\text {tor }} \rightarrow \mathrm{N}_{\kappa}^{\text {tor }}$ are log étale essentially by definition (see [Kato 1989, Theorem 3.5]). Moreover, as in [Faltings and Chai 1990, Chapter V, Remark 1.2(b)] and in the proof of [Lan 2008, Lemma 7.1.1.3], we have $R^{i}\left(f_{\kappa^{\prime}, \kappa}^{\text {tor }}\right)_{*} O_{\mathrm{N}_{\kappa^{\prime}}}^{\text {tor }}=0$ for $i>0$ by [Kempf et al. 1973, Chapter I, §3].

Lemma 3.1. Under the assumption that $\widetilde{\mathcal{H}}$ is neat, the closure of every stratum in $\tilde{\mathrm{M}}_{\widetilde{\mathcal{H}}, \widetilde{\Sigma}}^{\mathrm{tor}}$ has no self-intersection.

Proof. According to Definitions 1.33 and 1.34, the collection $\widetilde{\Sigma}$ of cone decompositions for $\widetilde{M}_{\widetilde{\mathcal{H}}}$ satisfies Condition 1.29. Hence [Lan 2008, Lemma 6.2.5.27 in the revision] implies that the closure of any stratum does not intersect itself. (See also [Faltings and Chai 1990, Chapter IV, Remark 5.8a].)

Corollary 3.2. For any $\kappa=(\widetilde{\mathcal{H}}, \widetilde{\Sigma}, \widetilde{\sigma}) \in \mathbf{K}_{Q, \mathcal{H}, \Sigma}^{\text {pre }}$, the closure $\mathrm{N}_{\kappa}^{\text {tor }}$ of $\mathrm{N}_{\kappa}=\widetilde{\mathrm{Z}}_{\left[\left(\widetilde{\Phi}_{\tilde{\mathcal{H}}}, \tilde{\delta}_{\tilde{\mathcal{H}}}, \widetilde{\sigma}\right)\right]}$ in $\widetilde{\mathrm{M}}_{\widetilde{\mathcal{H}}}^{\text {tor }}, \widetilde{\Sigma}$ is projective and smooth over $\mathrm{S}_{0}$, and the complement of $\mathrm{N}_{\kappa}$ in $\mathrm{N}_{\kappa}^{\text {tor }}$ (with its reduced structure) is a relative Cartier divisor with simple normal crossings. Thus the collection of open embeddings $\kappa^{\text {tor }}: \mathrm{N}_{\kappa} \hookrightarrow \mathrm{N}_{\kappa}^{\text {tor }}$, with $\kappa \in \mathbf{K}_{Q, \mathcal{H}, \Sigma}^{\mathrm{pr}}$, satisfies (1) of Theorem 2.15.

Proof. Combine Lemma 3.1 with (3) and (7) of Theorem 1.41.

From now on, let us fix a choice of $\kappa=(\widetilde{\mathcal{H}}, \widetilde{\Sigma}, \widetilde{\sigma}) \in \mathbf{K}_{Q, \mathcal{H}, \Sigma}^{\text {pre }}$, and suppress $\kappa$ and $\widetilde{\Sigma}$ from the notation. The compatibility of various objects under compositions with or pullbacks by $f_{\kappa^{\prime}, \kappa}^{\text {tor }}: \mathrm{N}_{\kappa^{\prime}}^{\text {tor }} \rightarrow \mathrm{N}_{\kappa}^{\text {tor }}$ (for $\kappa^{\prime} \succ \kappa$ in $\mathbf{K}_{Q, \mathcal{H}, \Sigma}^{\text {pre }}$ ) will be obvious from the constructions. 
3B. Extendability of structural morphisms. The goal of this subsection is to carry out steps (3) and (4) of Section 2C.

Let $\left(\widetilde{G}, \tilde{\lambda}, \tilde{i}, \widetilde{\alpha}_{\widetilde{\mathcal{H}}}\right)$ be the degenerating family of type $\widetilde{\mathrm{M}}_{\tilde{\mathcal{H}}}$ over $\tilde{\mathrm{M}}_{\mathcal{H}}^{\text {tor }}$. By construction of $\mathrm{N}$ as a boundary stratum of $\widetilde{\mathrm{M}}_{\widetilde{\mathcal{H}}}^{\text {tor }}$, the restriction $\widetilde{G}_{\mathrm{N}}$ of $\widetilde{G}$ to $\mathrm{N}$ is an extension of the pullback of the abelian scheme $G_{\mathrm{M}_{\mathcal{H}}}$ over $\mathrm{M}_{\mathcal{H}}$ to $\mathrm{N}$ by $f: \mathrm{N} \rightarrow \mathrm{M}_{\mathcal{H}}$, by the split torus $\widetilde{T}_{\mathrm{N}}$ over $\mathrm{N}$ with character group $\widetilde{X}$. The data of $\widetilde{\lambda}, \tilde{i}$, and $\widetilde{\alpha}_{\widetilde{\mathcal{H}}}$ induce respectively a polarization, an $\mathcal{O}$-endomorphism structure, and a level $\mathcal{H}$-structure on the abelian part of $\widetilde{G}_{\mathrm{N}}$, which agree with the pullbacks of the data $\lambda, i$, and $\alpha_{\mathcal{H}}$ over $\mathrm{M}_{\mathcal{H}}$ to $\mathrm{N}$ by $f: \mathrm{N} \rightarrow \mathrm{M}_{\mathcal{H}}$. By normality of (the closure) $\mathrm{N}^{\text {tor }}$ (of $\mathrm{N}$ in $\tilde{\mathrm{M}}_{\widetilde{\mathcal{H}}}^{\text {tor }}$ ), and by a result of Raynaud (see [Raynaud 1970, IX 2.4] or [Faltings and Chai 1990, Chapter I, Proposition 2.9]), the embedding $\widetilde{T}_{\mathrm{N}} \hookrightarrow \widetilde{G}_{\mathrm{N}}$ of group schemes extends (uniquely) to an embedding $\widetilde{T}_{\mathrm{N}^{\text {tor }}} \hookrightarrow \widetilde{G}_{\mathrm{N}^{\text {tor }}}$ of group schemes, and the quotient

$$
\bar{G}:=\widetilde{G}_{\mathrm{N}^{\mathrm{tor}}} / \widetilde{T}_{\mathrm{N}}{ }^{\text {tor }}
$$

is a semiabelian scheme whose restriction to $\mathrm{N}$ can be identified with the pullback of $G$ from $\mathrm{M}_{\mathcal{H}}$ to N. Similarly, we obtain $\bar{G}^{\vee}:=\widetilde{G}_{\mathrm{N}^{\text {tor }}}^{\vee} / \widetilde{T}_{\mathrm{N}^{\text {tor }}}^{\vee}$. By another result of Raynaud (see [Raynaud 1970, IX 1.4] or [Faltings and Chai 1990, Chapter I, Proposition 2.7]), the semiabelian $\bar{G}$ carries (unique) additional structures $\bar{\lambda}: \bar{G} \rightarrow \bar{G}^{\vee}$, $\bar{i}$, and $\bar{\alpha}_{\mathcal{H}}$ such that the restriction of $\left(\bar{G}, \bar{\lambda}, \bar{i}, \bar{\alpha}_{\mathcal{H}}\right)$ to $\mathrm{N}$ is the pullback of the tautological tuple over $\mathrm{M}_{\mathcal{H}}$ by $f: \mathrm{N} \rightarrow \mathrm{M}_{\mathcal{H}}$, and so that $\left(\bar{G}, \bar{\lambda}, \bar{i}, \bar{\alpha}_{\mathcal{H}}\right)$ defines a degenerating family of type $\mathrm{M}_{\mathcal{H}}$ over $\mathrm{N}^{\text {tor }}$.

Now the question is whether the structural morphism $f: \mathrm{N} \rightarrow \mathrm{M}_{\mathcal{H}}$ extends (necessarily uniquely) to a (proper) morphism $f^{\text {tor }}: \mathrm{N}^{\text {tor }} \rightarrow \mathrm{M}_{\mathcal{H}}^{\text {tor }}$ between the compactifications. By (6) of Theorem 1.41, this extendability can be verified after pullback to complete discrete valuation rings (with algebraically closed residue fields).

The stratification of $\tilde{\mathrm{M}}_{\tilde{\mathcal{H}}}^{\text {tor }}$ induces one on $\mathrm{N}^{\text {tor }}$. By (2) of Theorem 1.41, the strata of $\mathrm{N}^{\text {tor }}$ are parametrized by the faces of $\left[\left(\widetilde{\Phi}_{\widetilde{\mathcal{H}}}, \tilde{\delta}_{\widetilde{\mathcal{H}}}, \widetilde{\sigma}\right)\right]$ (as in Definition 1.38). Concretely, the faces of $\left[\left(\widetilde{\Phi}_{\widetilde{\mathcal{H}}}, \tilde{\delta}_{\widetilde{\mathcal{H}}}, \widetilde{\sigma}\right)\right]$ are equivalence classes $\left[\left(\breve{\Phi}_{\widetilde{\mathcal{H}}}, \breve{\delta}_{\widetilde{\mathcal{H}}}, \breve{\tau}\right)\right]$ of $\widetilde{\mathcal{H}}$-orbits of data of the following form:

(1) A fully symplectic admissible filtration $\breve{z}=\left\{\breve{Z}_{-i}\right\}$ on $\widetilde{L} \otimes_{\mathbb{Z}} \hat{\mathbb{Z}}^{\square}$ satisfying

$$
\widetilde{\mathrm{Z}}_{-2} \subset \breve{\mathrm{Z}}_{-2} \subset \breve{\mathrm{Z}}_{-1} \subset \widetilde{\mathrm{Z}}_{-1} \text {. }
$$

Any such filtration $\breve{Z}$ induces a fully symplectic admissible filtration $\mathrm{Z}=$ $\left\{\mathrm{Z}_{-i}\right\}$ on $L \otimes_{\mathbb{Z}} \hat{\mathbb{Z}}^{\square}$ by $\mathrm{Z}_{-2}:=\breve{\mathrm{Z}}_{-2} / \widetilde{\mathrm{Z}}_{-2}$ and $\mathrm{Z}_{-1}:=\breve{\mathrm{Z}}_{-1} / \widetilde{\mathrm{Z}}_{-2}$, so that there is a canonical isomorphism

$$
\mathrm{Z}_{0} / \mathrm{Z}_{-1} \cong \widetilde{\mathrm{Z}}_{-1} / \breve{\mathrm{Z}}_{-1}
$$


Conversely, any fully symplectic admissible filtration $\mathrm{z}$ on $L \otimes_{\mathbb{Z}} \hat{\mathbb{Z}}^{\square}$ induces a fully symplectic admissible filtration $\breve{Z}$ on $\widetilde{L} \otimes_{\mathbb{Z}} \hat{\mathbb{Z}}^{\square}$ satisfying (3.3) and (3.4).

(2) A torus argument $\breve{\Phi}=\left(\breve{X}, \breve{Y}, \breve{\phi}, \breve{\varphi}_{-2}, \breve{\varphi}_{0}\right)$ for $\breve{Z}$ (as in Definition 1.13), together with admissible surjections $s_{\breve{X}}: \breve{X} \rightarrow \widetilde{X}$ and $s_{\breve{Y}}: \breve{Y} \rightarrow \widetilde{Y}$ satisfying $s_{\breve{X}} \breve{\phi}=\widetilde{\phi} s_{\breve{Y}}$ and other natural compatibilities with $\breve{\varphi}_{-2}, \breve{\varphi}_{0}, \widetilde{\varphi}_{-2}$, and $\widetilde{\varphi}_{0}$. (See Definitions 1.18-1.20.)

Any $\breve{\Phi}, s_{\breve{X}}$, and $s_{\breve{Y}}$ determine a torus argument $\Phi=\left(X, Y, \phi, \varphi_{-2}, \varphi_{0}\right)$ for $\mathrm{Z}$ by $X:=\operatorname{ker}\left(s_{\breve{X}}\right), Y:=\operatorname{ker}\left(s_{\breve{Y}}\right)$, and $\phi:=\left.\breve{\phi}\right|_{Y}$, so that there is a commutative diagram

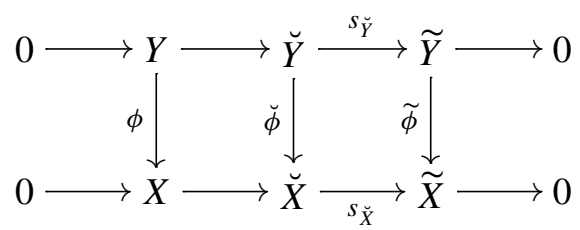

whose horizontal rows are exact sequences.

(3) The existence of some splitting of $\breve{Z}$, inducing some liftable splitting $\breve{\delta}_{\widetilde{\mathcal{H}}}$ defining the cusp label $\left(\breve{Z}_{\widetilde{\mathcal{H}}}, \breve{\Phi}_{\widetilde{\mathcal{H}}}, \breve{\delta}_{\widetilde{\mathcal{H}}}\right)$ at level $\widetilde{\mathcal{H}}$.

Given the liftable splitting $\tilde{\delta}_{\widetilde{\mathcal{H}}}$, the existence of the liftable splitting $\breve{\delta}_{\widetilde{\mathcal{H}}}$ is equivalent to the existence of some liftable splitting $\delta_{\mathcal{H}}$ of $Z_{\mathcal{H}}$. Then we see that there is a canonical bijection between cusp labels $\left[\left(\mathrm{Z}_{\mathcal{H}}, \Phi_{\mathcal{H}}, \delta_{\mathcal{H}}\right)\right]$ at level $\mathcal{H}$ and cusp labels $\left[\left(\breve{\mathrm{Z}}_{\widetilde{\mathcal{H}}}, \breve{\Phi}_{\widetilde{\mathcal{H}}}, \breve{\delta}_{\widetilde{\mathcal{H}}}\right)\right]$ at level $\widetilde{\mathcal{H}}$ admitting a surjection to $\left.\left[\widetilde{\mathrm{Z}}_{\widetilde{\mathcal{H}}}, \widetilde{\Phi}_{\widetilde{\mathcal{H}}}, \tilde{\delta}_{\widetilde{\mathcal{H}}}\right)\right]$.

(4) Let $\Phi_{\mathcal{H}}$ (resp. $\breve{\Phi}_{\widetilde{\mathcal{H}}}$ ) be the torus argument for $\mathrm{Z}_{\mathcal{H}}$ (resp. $\widetilde{\mathrm{Z}}_{\widetilde{\mathcal{H}}}$ ) at level $\mathcal{H}$ (resp. $\widetilde{\mathcal{H}}$ ) induced by $\Phi$ (resp. $\breve{\Phi}$ ). Then (3.5) induces morphisms

$$
\mathbf{S}_{\Phi_{\mathcal{H}}} \hookrightarrow \mathbf{S}_{\breve{\Phi}_{\widetilde{\mathcal{H}}}} \rightarrow \mathbf{S}_{\widetilde{\Phi}_{\tilde{\mathcal{H}}}},
$$

where the first morphism is canonical, and where the second morphism is defined by $s_{\breve{X}}$ and $s_{\breve{Y}}$, whose composition is zero. (In general, the morphisms in (3.6) do not form an exact sequence.)

The dual of (3.6) defines morphisms

$$
\mathbf{P}_{\widetilde{\Phi}_{\tilde{\mathcal{H}}}}^{+} \hookrightarrow \mathbf{P}_{\breve{\Phi}_{\tilde{\mathcal{H}}}} \rightarrow \mathbf{P}_{\Phi_{\mathcal{H}}},
$$

where the first morphism is defined by $s_{\breve{X}}$ and $s_{\breve{Y}}$, and where the second morphism is canonical, whose composition is zero.

Then $\breve{\tau} \subset \mathbf{P}_{\breve{\Phi}_{\tilde{\mathcal{H}}}}^{+}$is a cone in the cone decomposition $\widetilde{\Sigma}_{\breve{\Phi}_{\tilde{\mathcal{H}}}}$ having a face $\breve{\sigma}$ that is a $\Gamma_{\breve{\Phi}_{\widetilde{\mathcal{H}}}}$-translation (see Definition 1.23) of the image of $\widetilde{\sigma} \subset \mathbf{P}_{\widetilde{\Phi}_{\tilde{\mathcal{H}}}}^{+}$under the first morphism in (3.7). 
By (5) of Theorem 1.41, the formal completion

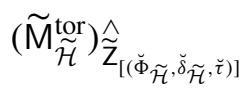

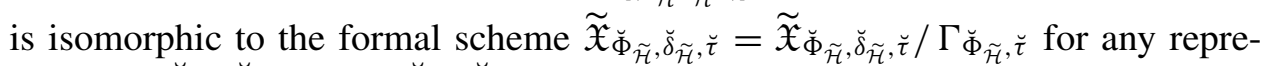

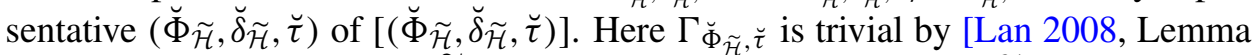
6.2.5.27 in the revision], and $\widetilde{\mathfrak{X}}_{\Phi_{\tilde{\mathcal{H}}}, \breve{\delta}_{\widetilde{\mathcal{H}}}, \breve{\tau}}$ is the formal completion of $\widetilde{\Xi}_{\Phi_{\tilde{\mathcal{H}}}, \breve{\delta}_{\widetilde{\mathcal{H}}}}(\breve{\tau})$ along its $\breve{\tau}$-stratum $\left(\widetilde{\Xi}_{\breve{\Phi}_{\tilde{\mathcal{H}}}, \breve{\delta}_{\tilde{\mathcal{H}}}}\right)_{\bar{\tau}}$.

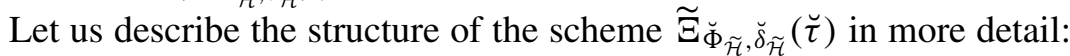

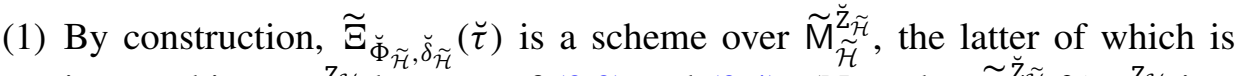
isomorphic to $\mathrm{M}_{\mathcal{H}}^{Z_{\mathcal{H}}}$ because of (3.3) and (3.4). (Note that $\widetilde{\mathrm{M}}_{\widetilde{\mathcal{H}}}^{\check{Z}_{\widetilde{\mathcal{H}}}} \cong \mathrm{M}_{\mathcal{H}}^{Z_{\mathcal{H}}}$ is a scheme by [Lan 2008, Corollary 7.2.3.10].)

By abuse of notation, we shall simply denote the push-forward

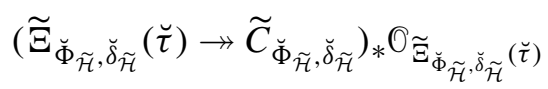

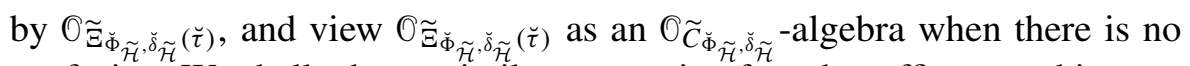
confusion. We shall adopt a similar convention for other affine morphisms.

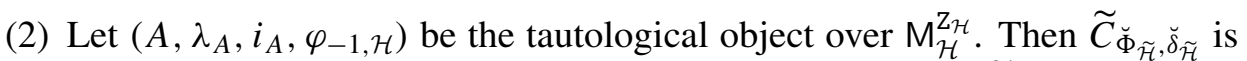
the abelian scheme over $\mathrm{M}_{\mathcal{H}}$ parametrizing liftings (to level $\tilde{\mathcal{H}}$ ) of data of the form $\left(\breve{c}: \breve{X} \rightarrow A^{\vee}, \breve{c}^{\vee}: \breve{Y} \rightarrow A\right.$ ), compatible with $\breve{\phi}: \breve{Y} \hookrightarrow \breve{X}$ and satisfying certain liftability and pairing conditions (coming from the so-called symplecticliftability on the level structures). By construction, $\widetilde{C}_{\breve{\Phi}_{\widetilde{\mathcal{H}}}, \breve{\delta}_{\widetilde{\mathcal{H}}}}$ is $\mathbb{Z}_{(\square)}^{\times}$-isogenous to $\underline{\operatorname{Hom}}_{\mathcal{O}}(\breve{Y}, A)^{\circ}$.

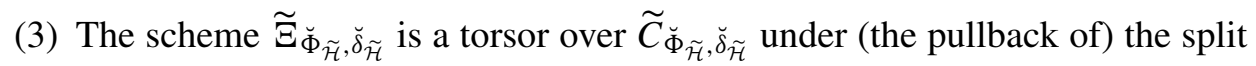
torus $E_{\breve{\Phi}_{\tilde{\mathcal{H}}}}=\underline{\operatorname{Hom}}\left(\mathbf{S}_{\Phi_{\tilde{\mathcal{H}}}}, \mathbf{G}_{\mathrm{m}}\right)$, which can be identified with the relative spectrum

$$
\operatorname{Spec}_{\widetilde{C}_{\tilde{\Phi}_{\tilde{\mathcal{H}}}, \breve{\delta}_{\tilde{\mathcal{H}}}}}\left(\bigoplus_{\breve{\ell} \in \mathbf{S}_{\check{\Phi}_{\tilde{\mathcal{H}}}}} \widetilde{\Psi}_{\check{\Phi}_{\tilde{\mathcal{H}}}, \breve{\delta}_{\tilde{\mathcal{H}}}}(\breve{\ell})\right),
$$

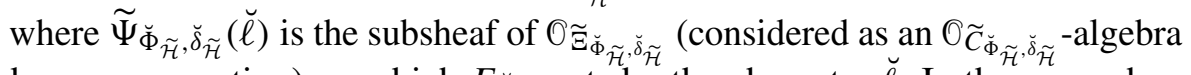
by our convention) on which $E_{\breve{\Phi}_{\tilde{\mathcal{H}}}}$ acts by the character $\breve{\ell}$. In the case when $\breve{\ell}=[\breve{y} \otimes \breve{\chi}]$, where $\breve{y} \in \breve{Y}$ and $\breve{\chi} \in \breve{X}$, there is a canonical identification

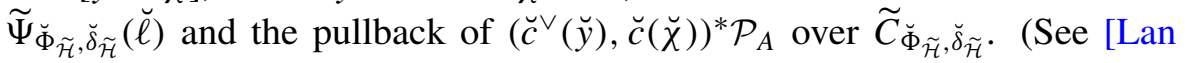
2008, Convention 6.2.3.26 and end of §6.2.4].)

(4) Consider the subsemigroups of $\mathbf{S}_{\breve{\Phi}_{\widetilde{\mathcal{H}}}}$ (see [Lan 2008, Definitions 6.1.1.9 and 6.1.2.5]) given by

$$
\begin{aligned}
& \breve{\tau}^{\vee}=\left\{\breve{\ell} \in \mathbf{S}_{\breve{\Phi}_{\tilde{\mathcal{H}}}}:\langle\breve{\ell}, y\rangle \geq 0, \forall y \in \breve{\tau}\right\}, \\
& \breve{\tau}_{0}^{\vee}=\left\{\breve{\ell} \in \mathbf{S}_{\breve{\Phi}_{\tilde{\mathcal{H}}}}:\langle\breve{\ell}, y\rangle>0, \forall y \in \breve{\tau}\right\}, \\
& \breve{\tau}^{\perp}=\left\{\breve{\ell} \in \mathbf{S}_{\breve{\Phi}_{\tilde{\mathcal{H}}}}:\langle\breve{\ell}, y\rangle=0, \forall y \in \breve{\tau}\right\} \cong \breve{\tau}^{\vee} / \breve{\tau}_{0}^{\vee} .
\end{aligned}
$$




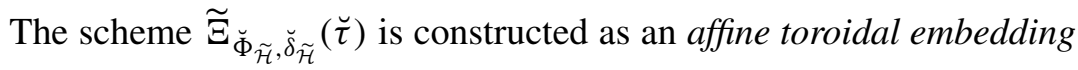

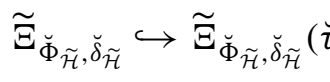

along $\breve{\tau}$ over the abelian scheme $\widetilde{C}_{\breve{\Phi}_{\tilde{\mathcal{H}}}, \breve{\delta}_{\widetilde{\mathcal{H}}}}$, which can be identified with the relative spectrum

$$
\operatorname{Spec}_{\widetilde{C}_{\widetilde{\Phi}_{\tilde{\mathcal{H}}}, \breve{\delta}_{\tilde{\mathcal{H}}}}}\left(\bigoplus_{\breve{\ell} \in \breve{\tau}^{\vee}} \widetilde{\Psi}_{\breve{\Phi}_{\tilde{\mathcal{H}}}, \breve{\delta}_{\tilde{\mathcal{H}}}}(\breve{\ell})\right) .
$$

(5) Finally, the sheaf of ideals

$$
\tilde{\Phi}_{\breve{\tau}}=\bigoplus_{\breve{\ell} \in \breve{\tau}_{0}^{\vee}} \widetilde{\Psi}_{\breve{\Phi}_{\tilde{\mathcal{H}}}, \breve{\delta}_{\tilde{\mathcal{H}}}}(\breve{\ell})
$$

(see [Lan 2008, Lemma 6.1.2.6]) defines the $\breve{\tau}$-stratum $\left(\widetilde{\Xi}_{\breve{\Phi}_{\widetilde{\mathcal{H}}}, \breve{\delta}_{\widetilde{\mathcal{H}}}}\right)_{\breve{\tau}}$, which can be identified with the relative spectrum

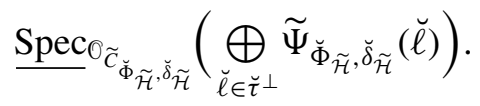

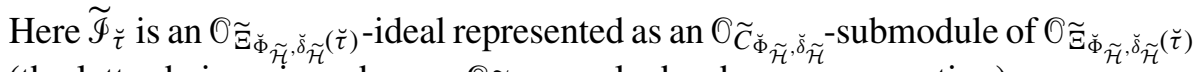
(the latter being viewed as an $0 \widetilde{C}_{\check{\Phi}_{\tilde{\mathcal{H}}}, \check{\delta}_{\tilde{\mathcal{H}}}}$-algebra by our convention).

Suppose $\breve{\sigma}$ is the face of $\breve{\tau}$ that is a $\Gamma_{\Phi_{\tilde{\mathcal{H}}}}$-translation of the image of $\widetilde{\sigma} \subset \mathbf{P}_{\widetilde{\Phi}_{\tilde{\mathcal{H}}}}^{+}$ under the first morphism in (3.7). Similar to the definition of $\breve{\tau}^{\vee}, \breve{\tau}_{0}^{\vee}$, and $\breve{\tau}^{\perp}$ above, consider the following subsemigroups of $\mathbf{S}_{\breve{\Phi}_{\tilde{\mathcal{H}}}}$ :

$$
\begin{aligned}
& \breve{\sigma}^{\vee}=\left\{\breve{\ell} \in \mathbf{S}_{\breve{\Phi}_{\tilde{\mathcal{H}}}}:\langle\breve{\ell}, y\rangle \geq 0, \forall y \in \breve{\sigma}\right\}, \\
& \breve{\sigma}_{0}^{\vee}=\left\{\breve{\ell} \in \mathbf{S}_{\breve{\Phi}_{\tilde{\mathcal{H}}}}:\langle\breve{\ell}, y\rangle>0, \forall y \in \breve{\sigma}\right\}, \\
& \breve{\sigma}^{\perp}=\left\{\breve{\ell} \in \mathbf{S}_{\breve{\Phi}_{\tilde{\mathcal{H}}}}:\langle\breve{\ell}, y\rangle=0, \forall y \in \breve{\sigma}\right\} \cong \breve{\sigma}^{\vee} / \breve{\sigma}_{0}^{\vee} .
\end{aligned}
$$

Note that $\breve{\tau}^{\vee} \subset \breve{\sigma}^{\vee}$ and $\breve{\tau}^{\perp} \subset \breve{\sigma}^{\perp}$, but $\breve{\tau}_{0}^{\vee} \not \subset \breve{\sigma}_{0}^{\vee}$ in general. The closure $\left(\widetilde{\Xi}_{\breve{\Phi}_{\widetilde{\mathcal{H}}}, \breve{\delta}_{\widetilde{\mathcal{H}}}}\right)_{\breve{\sigma}}(\breve{\tau})$

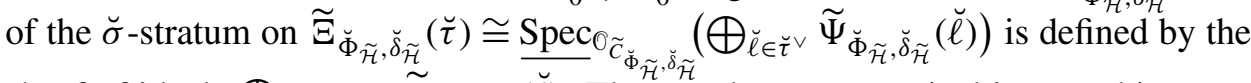
sheaf of ideals $\bigoplus_{\breve{\ell} \in \breve{\sigma}_{0}^{\vee} \cap \breve{\tau}^{\vee}} \widetilde{\Psi}_{\breve{\Phi}_{\tilde{\mathcal{H}}}, \breve{\delta}_{\widetilde{\mathcal{H}}}}(\breve{\ell})$. Then we have a canonical isomorphism

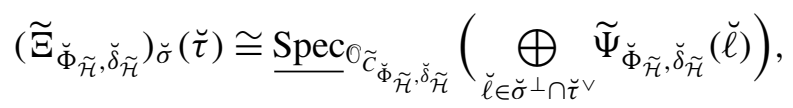

with the $\breve{\tau}$-stratum

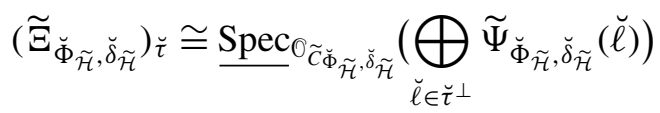

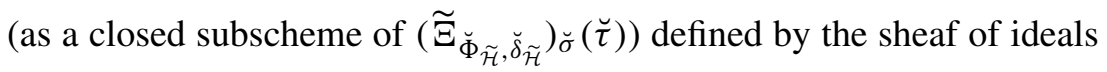

$$
\tilde{\Phi}_{\breve{\sigma}, \breve{\tau}}:=\bigoplus_{\breve{\ell} \in \breve{\sigma}^{\perp} \cap \breve{\tau}_{0}^{v}} \widetilde{\Psi}_{\breve{\Phi}_{\widetilde{\mathcal{H}}}, \breve{\delta}_{\widetilde{\mathcal{H}}}}(\breve{\ell}) .
$$




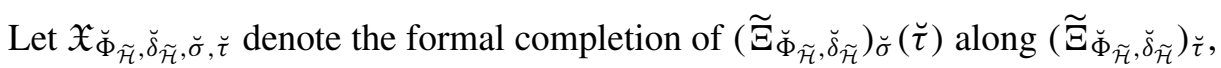

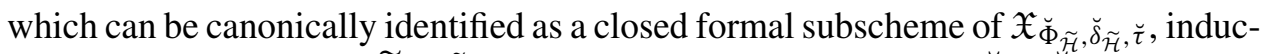
ing the closures of the $\left[\left(\widetilde{\Phi}_{\widetilde{\mathcal{H}}}, \tilde{\delta}_{\tilde{\mathcal{H}}}, \widetilde{\sigma}\right)\right]$-strata on any good formal $\left(\breve{\Phi}_{\tilde{\mathcal{H}}}, \bar{\delta}_{\tilde{\mathcal{H}}}, \breve{\tau}\right)$-model. (See [Lan 2008, Definition 6.3.1.11] for the definition of good formal models, and see [Lan 2008, Definition 6.3.2.16 in the revision] for the labeling of the strata by equivalence classes of triples of the form $\left[\left(\widetilde{\Phi}_{\widetilde{\mathcal{H}}}, \tilde{\delta}_{\tilde{\mathcal{H}}}, \widetilde{\sigma}\right)\right]$.) By (5) of Theorem 1.41,

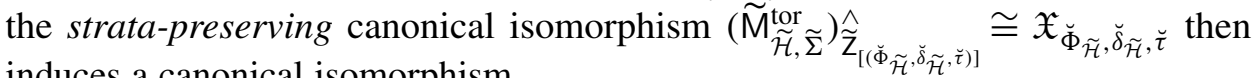
induces a canonical isomorphism

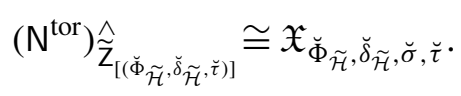

(Alternatively, one may refer directly to the gluing construction of $\widetilde{\mathrm{M}}_{\widetilde{\mathcal{H}}}^{\text {tor }}$ in [Lan 2008, §6.3.3], based on the crucial [Lan 2008, Proposition 6.3.2.13].)

By the theory of two-step constructions (see [Faltings and Chai 1990, Chapter III Theorem 10.2] and [Lan 2008, §4.5.6 in the revision]), the degeneration data of the pullback of $\left(\bar{G}, \bar{\lambda}, \bar{i}, \bar{\alpha}_{\mathcal{H}}\right)$ to affine open formal subschemes of $\mathfrak{X}_{\breve{\Phi}_{\tilde{\mathcal{H}}}, \breve{\delta}_{\tilde{\mathcal{H}}}, \breve{\sigma}, \breve{\tau}}$ can be obtained from the degeneration data of pullback of $\left(\widetilde{G}, \tilde{\lambda}, \tilde{i}, \widetilde{\alpha}_{\mathcal{H}}\right)$ to affine open formal subschemes of $\widetilde{\mathfrak{X}}_{\Phi_{\tilde{\mathcal{H}}}, \breve{\delta}_{\tilde{\mathcal{H}}}, \breve{\tau}}$ by restricting objects defined on $\breve{X}$ and $\breve{Y}$ to the subgroups $X$ and $Y$. Therefore, in order to verify (6) of Theorem 1.41, it suffices to verify the following:

Condition 3.8 (cf. [Faltings and Chai 1990, Chapter VI, Definition 1.3]). For each $\left(\breve{\Phi}_{\widetilde{\mathcal{H}}}, \breve{\delta}_{\widetilde{\mathcal{H}}}, \breve{\tau}\right)$ as above, the image of $\breve{\tau}$ in $\mathbf{P}_{\Phi_{\mathcal{H}}}$ under the (canonical) second morphism in (3.7) is contained in some cone $\tau \subset \mathbf{P}_{\Phi_{\mathcal{H}}}^{+}$in the cone decomposition $\Sigma_{\Phi_{\mathcal{H}}}$

If Condition 3.8 is satisfied (for $\kappa=(\widetilde{\mathcal{H}}, \widetilde{\Sigma}, \widetilde{\sigma}))$, then the structural morphism $f: \mathrm{N} \rightarrow \mathrm{M}_{\mathcal{H}}$ extends to a (unique) morphism $f^{\text {tor }}: \mathrm{N}^{\text {tor }} \rightarrow \mathrm{M}_{\mathcal{H}}^{\text {tor }}$, which is étale locally given by morphisms between toric schemes equivariant under (surjective) morphisms between tori. By construction, we have a commutative diagram

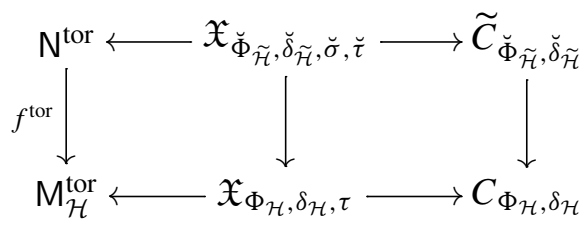

of canonical morphisms whenever the image of $\breve{\tau}$ under the (canonical) second morphism in (3.7) is contained in $\tau$.

Remark 3.10. Condition 3.8 is analogous to the condition in [Pink 1990, 6.25(b)], used in for example [Harris and Zucker 1994, Lemma 1.6.5] and related works based on [Ash et al. 1975]. Unfortunately, we must point out that, apart from some pleasant (and often suggestive) analogies, there is no logical implication between 
the analytic theory in [Ash et al. 1975; Pink 1990], and the algebraic theory in [Faltings and Chai 1990; Lan 2008]. (One cannot even use $G(\mathbb{Q})$ in the algebraic theory.) The applicability of Condition 3.8 in our work cannot be proved using [Pink 1990, 6.25(b)].

As planned in step (4) of Section $2 \mathrm{C}$, let us take $\mathbf{K}_{Q, \mathcal{H}, \Sigma}$ to be the subset of $\mathbf{K}_{Q, \mathcal{H}, \Sigma}^{\mathrm{pre}}$ consisting of elements $\kappa$ satisfying Condition 3.8. Since Condition 3.8 can be achieved by replacing any given $\widetilde{\Sigma}$ with a refinement, we see that $\mathbf{K}_{Q, \mathcal{H}, \Sigma}$ is nonempty and has an induced directed partial order.

From now on, assume that our fixed choice $\kappa=(\widetilde{\mathcal{H}}, \widetilde{\Sigma}, \widetilde{\sigma})$ lies in $\mathbf{K}_{Q, \mathcal{H}, \Sigma}$.

3C. Logarithmic smoothness of $f^{\text {tor }}$. The aim of this subsection is to carry out step (5) of Section 2C.

We need to show that the morphism $f^{\text {tor }}$ is $\log$ smooth (as in [Kato 1989, 3.3] and [Illusie 1994, 1.6]) if we equip $\mathrm{N}^{\text {tor }}$ and $\mathrm{M}_{\mathcal{H}}^{\text {tor }}$ with the canonical fine log structures given respectively by the relative Cartier divisors with simple normal crossings given by the complements $\mathrm{N}^{\text {tor }}-\mathrm{N}$ and $\mathrm{M}_{\mathcal{H}}^{\text {tor }}-\mathrm{M}_{\mathcal{H}}$ with their reduced structures. According to [Kato 1989, 3.12], we have the following:

Lemma 3.11. To show that the morphism $f^{\text {tor }}$ is log smooth, it suffices to show that the first morphism in the canonical exact sequence

$$
\left(f^{\text {tor }}\right)^{*}\left(\Omega_{\mathrm{M}_{\mathcal{H}}^{\text {tor }} / \mathrm{S}_{0}}^{1}[d \log \infty]\right) \rightarrow \Omega_{\mathrm{N}^{\text {tor }} / \mathrm{S}_{0}}^{1}[d \log \infty] \rightarrow \bar{\Omega}_{\mathrm{N}^{\text {tor }} / \mathrm{M}_{\mathcal{H}}^{\text {tor }}}^{1} \rightarrow 0
$$

is injective, and that $\bar{\Omega}_{\mathrm{N}^{\text {tor }} / \mathrm{M}_{\mathcal{H}}^{\text {tor }}}$ is locally free of finite rank.

By (4) of Theorem 1.41, the extended Kodaira-Spencer morphism [Lan 2008, Definition 4.6.3.32] for $G \rightarrow \mathrm{M}_{\mathcal{H}}^{\text {tor }}$ induces an isomorphism

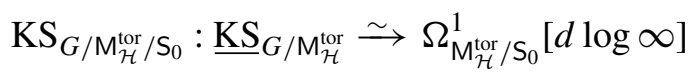

over $\mathrm{M}_{\mathcal{H}}^{\text {tor }}$, while the extended Kodaira-Spencer morphism for $\widetilde{G} \rightarrow \widetilde{\mathrm{M}}_{\widetilde{\mathcal{H}}}^{\text {tor }}$ induces an isomorphism

$$
\mathrm{KS} \widetilde{G} / \widetilde{\mathrm{M}}_{\tilde{\mathcal{H}}}^{\text {tor }} / \mathrm{S}_{0}: \underline{\mathrm{KS}} \widetilde{G} / \widetilde{\mathrm{M}}_{\tilde{\mathcal{H}}}^{\text {tor }} \stackrel{\sim}{\longrightarrow} \Omega_{\widetilde{\mathrm{M}}_{\tilde{\mathcal{H}}}^{\text {tor }} / \mathrm{S}_{0}}^{1}[d \log \infty]
$$

over $\widetilde{\mathrm{M}}_{\widetilde{\mathcal{H}}}^{\text {tor }}$. Over $\mathrm{N}^{\text {tor }}$, we have canonical extensions

$$
0 \rightarrow \widetilde{T}_{\mathrm{N}^{\text {tor }}} \rightarrow \widetilde{G}_{\mathrm{N}^{\text {tor }}} \rightarrow \bar{G} \rightarrow 0 \quad \text { and } \quad 0 \rightarrow \widetilde{T}_{\mathrm{N} \text { tor }}^{\vee} \rightarrow \widetilde{G}_{\mathrm{N}^{\text {tor }}}^{\vee} \rightarrow \bar{G}^{\vee} \rightarrow 0
$$

of group schemes, inducing exact sequences

$$
0 \rightarrow \mathrm{Lie}_{\bar{G} / \mathrm{N}^{\text {tor }}}^{\vee} \rightarrow \mathrm{Lie}_{\widetilde{G}_{\mathrm{N}^{\text {tor }}} / \mathrm{N}^{\text {tor }}}^{\vee} \rightarrow \mathrm{Lie}_{\widetilde{T}_{\mathrm{N}^{\text {tor }}} / \mathrm{N}^{\text {tor }}} \rightarrow 0
$$

and

$$
0 \rightarrow \mathrm{Lie}_{\bar{G}^{\vee} / \mathrm{N}^{\text {tor }}}^{\vee} \rightarrow \mathrm{Lie}_{\widetilde{G}_{\mathrm{N}^{\text {tor }}}^{\vee} / \mathrm{N}^{\text {tor }}}^{\vee} \rightarrow \mathrm{Lie}_{\widetilde{T}_{\mathrm{N}}^{\vee}}^{\vee} / \mathrm{N}^{\text {tor }} \rightarrow 0
$$


Therefore, there is a canonical surjection

$$
\underline{\mathrm{KS}} \widetilde{G}_{\mathrm{N}^{\text {tor }} / \mathrm{N}^{\text {tor }}} \rightarrow \underline{\mathrm{KS}} \widetilde{T}_{\mathrm{N}_{\text {tor }} / \mathrm{N}^{\text {tor }}},
$$

where $\underline{\mathrm{KS}_{\widetilde{T}_{\mathrm{N}}} / \mathrm{N}^{\text {tor }}}$ is the pullback of the sheaf

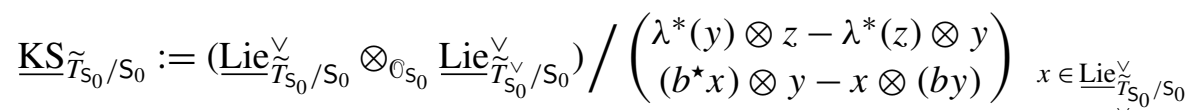

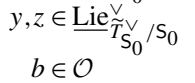

defined (as for degenerating families in Definition 1.40) by the split tori $\widetilde{T}$ and $\widetilde{T}^{\vee}$ over $\mathrm{S}_{0}$ with respective character groups $\tilde{X}$ and $\tilde{Y}$. The kernel

$$
\underline{\mathrm{K}}:=\operatorname{ker}\left(\underline{\mathrm{KS}} \widetilde{G}_{\mathrm{N}^{\text {tor }}} / \mathrm{N}^{\text {tor }} \rightarrow \underline{\mathrm{KS}} \widetilde{T}_{\mathrm{N}^{\text {tor }}} / \mathrm{N}^{\text {tor }}\right)
$$

contains $\underline{\mathrm{KS}}_{\bar{G} / \mathrm{N}^{\text {tor }}}$ as a natural subsheaf, and the quotient of $\underline{\mathrm{K}}$ by $\underline{\mathrm{KS}}_{\bar{G} / \mathrm{N}^{\text {tor }}}$ is isomorphic to

$$
\begin{aligned}
& \left(\underline{\operatorname{Lie}}_{\bar{G} / \mathrm{N}^{\text {tor }}}^{\vee} \otimes_{\mathcal{O}_{\text {Ntor }}} \underline{\operatorname{Lie}}_{\widetilde{T}_{\text {Ntor }}^{\vee} / \mathrm{N}^{\text {tor }}}^{v}\right) /\left(\left(b^{\star} x\right) \otimes y-x \otimes(b y)\right)_{x \in \underline{\operatorname{Lie}}_{\bar{G} / N^{\text {tor }}}^{\vee}} \\
& y \in \underline{\mathrm{Lie}}_{\widetilde{T}_{\mathrm{N}}^{\vee}}^{\vee} / \mathrm{N}^{\text {tor }} \\
& b \in \mathcal{O} \\
& \cong \underline{\operatorname{Hom}}_{\mathcal{O}} \otimes_{\mathbb{Z}} \mathcal{O}_{\text {Nor }}\left(\underline{\operatorname{Lie}}_{\mathrm{T}_{\text {Nor }}^{\vee}}^{\vee} / \mathrm{N}^{\text {tor }}, \underline{\mathrm{Lie}}_{\bar{G} / \mathrm{N}^{\text {tor }}}^{\vee}\right)
\end{aligned}
$$

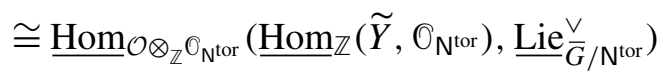

$$
\begin{aligned}
& \cong \underline{\operatorname{Hom}}_{\mathcal{O}}\left(\widetilde{Y}^{\vee}, \underline{\operatorname{Lie}}_{\bar{G} / \mathrm{N}^{\text {tor }}}^{\vee}\right) \\
& \cong \underline{\operatorname{Hom}}_{\mathcal{O}}\left(Q^{\vee}, \underline{\operatorname{Lie}}_{\bar{G} / \mathrm{N}^{\text {tor }}}\right) \text {. }
\end{aligned}
$$

Since the pullback of $\left(G, \lambda, i, \alpha_{\mathcal{H}}\right)$ under $\mathrm{N}^{\text {tor }} \rightarrow \mathrm{M}_{\mathcal{H}}^{\text {tor }}$ is isomorphic to $\left(\bar{G}, \bar{\lambda}, \bar{i}, \bar{\alpha}_{\mathcal{H}}\right)$, we have canonical isomorphisms

$$
\left(f^{\text {tor }}\right)^{*} \underline{\mathrm{KS}}_{G / \mathrm{M}_{\mathcal{H}}^{\text {tor }}} \cong \underline{\mathrm{KS}}_{\bar{G} / \mathrm{N}^{\text {tor }}}
$$

and

$$
\left(f^{\text {tor }}\right)^{*}\left(\underline{\operatorname{Hom}}_{\mathcal{O}}\left(Q^{\vee}, \underline{\operatorname{Lie}}_{G / \mathrm{M}_{\mathcal{H}}^{\text {tor }}}^{\vee}\right)\right) \cong \underline{\operatorname{Hom}}_{\mathcal{O}}\left(Q^{\vee}, \underline{\operatorname{Lie}}_{\bar{G} / \mathrm{N}^{\text {tor }}}^{\vee}\right) .
$$

Since the étale local structure of $\widetilde{\mathrm{M}}_{\widetilde{\mathcal{H}}}^{\text {tor }}$ along the $\left[\left(\breve{\Phi}_{\widetilde{\mathcal{H}}}, \breve{\delta}_{\widetilde{\mathcal{H}}}, \breve{\tau}\right)\right]$-stratum is the same as

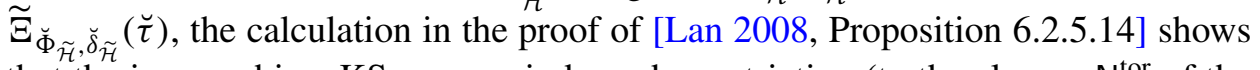
that the isomorphism $\mathrm{KS}_{\widetilde{G}} / \widetilde{\mathrm{M}}_{\widetilde{\mathcal{H}}}^{\text {tor }} / \mathrm{S}_{0}$ induces by restriction (to the closure $\mathrm{N}^{\text {tor }}$ of the $\left[\left(\widetilde{\Phi}_{\widetilde{\mathcal{H}}}, \tilde{\delta}_{\widetilde{\mathcal{H}}}, \widetilde{\sigma}\right)\right]$-stratum) an isomorphism

$$
\underline{\mathrm{K}} \stackrel{\sim}{\longrightarrow} \Omega_{\mathrm{N}^{\mathrm{tor}} / \mathrm{S}_{0}}^{1}[d \log \infty]
$$


making the diagram

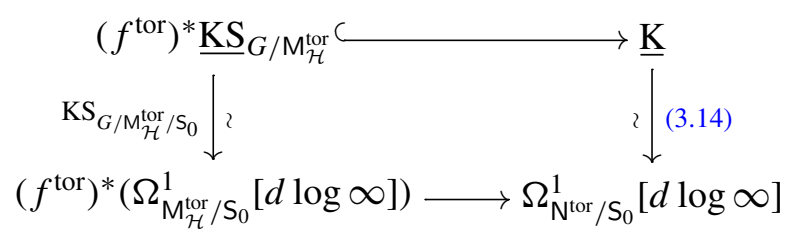

commutative. In particular, the bottom arrow (which is the first morphism in (3.12)) is injective, and the isomorphism (3.14) induces a canonical isomorphism

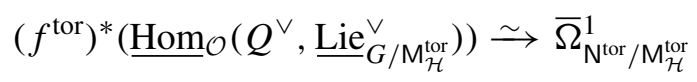

of coherent sheaves over $\mathrm{N}^{\text {tor }}$. (The restriction of (3.15) to $\mathrm{N}$ is compatible with the composition of isomorphisms (2.17) because of the same calculation in the proof of [Lan 2008, Proposition 6.2.5.14].)

Thus the desired isomorphism (2.16) is a consequence of (3.15). Moreover, since $\underline{\operatorname{Hom}}_{\mathcal{O}}\left(Q^{\vee},{\underline{\mathrm{Lie}^{-}}}_{G / \mathrm{M}_{\mathcal{H}}^{\text {tor }}}\right)$ (see Remark 2.14) is locally free of finite rank over $\mathrm{M}_{\mathcal{H}}^{\text {tor }}$, the isomorphism (3.15) shows that the sheaf $\bar{\Omega}_{\mathrm{N}^{\text {tor }} / \mathrm{M}_{\mathcal{H}}^{\text {tor }}}^{1}$ is also locally free of finite rank over $\mathrm{N}^{\text {tor }}$. By Lemma 3.11, this shows that $f^{\text {tor }}{ }_{1 \text { is }} \log$ smooth, and completes the proof of (2) and (3a) of Theorem 2.15.

3D. Equidimensionality of $\boldsymbol{f}^{\text {tor }}$. Let us take a closer look at the diagram (3.9). By construction of $f^{\text {tor }}$, given any stratum $\mathrm{Z}_{\left[\left(\Phi_{\mathcal{H}}, \delta_{\mathcal{H}}, \tau\right)\right]}$ of $\mathrm{M}_{\mathcal{H}}^{\text {tor }}$, the preimage

$$
\widetilde{\mathrm{Z}}_{\left[\left(\Phi_{\mathcal{H}}, \delta_{\mathcal{H}}, \tau\right)\right]}:=\left(f^{\text {tor }}\right)^{-1}\left(\mathrm{Z}_{\left[\left(\Phi_{\mathcal{H}}, \delta_{\mathcal{H}}, \tau\right)\right]}\right)
$$

has a stratification formed by $\widetilde{Z}_{\left[\left(\breve{\Phi}_{\tilde{\mathcal{H}}}, \breve{\delta}_{\tilde{\mathcal{H}}}, \breve{\tau}\right)\right]}$, where $\breve{\tau}$ runs through cones in $\widetilde{\Sigma}_{\breve{\Phi}_{\widetilde{\mathcal{H}}}}$ satisfying the following conditions:

(1) $\breve{\tau} \subset \mathbf{P}_{\breve{\Phi}_{\widetilde{\mathcal{H}}}^{+}}^{+}$.

(2) $\breve{\tau}$ has a face $\breve{\sigma}$ that is a $\Gamma_{\widetilde{\Phi}_{\tilde{\mathcal{H}}}}$-translation of the image of $\widetilde{\sigma} \subset \mathbf{P}_{\widetilde{\Phi}_{\tilde{\mathcal{H}}}}^{+}$under the first morphism in (3.7).

(3) The image of $\breve{\tau}$ under the (canonical) second morphism in (3.7) is contained in $\tau \subset \mathbf{P}_{\Phi_{\mathcal{H}}}^{+}$.

The formal completion $\left(\mathrm{N}^{\text {tor }}\right)_{\tilde{\mathrm{Z}}_{\left[\left(\Phi_{\mathcal{H}}, \delta_{\mathcal{H}}, \tau\right)\right]}}$ admits a canonical morphism

$$
\left(\mathrm{N}^{\mathrm{tor}}\right)_{\widetilde{\mathrm{Z}}_{\left[\left(\Phi_{\mathcal{H}}, \delta_{\mathcal{H}}, \tau\right)\right]}} \rightarrow C_{\Phi_{\mathcal{H}}, \delta_{\mathcal{H}}},
$$

whose precomposition with the canonical morphism

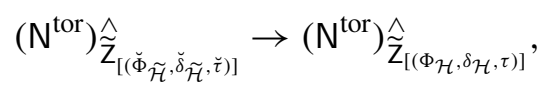




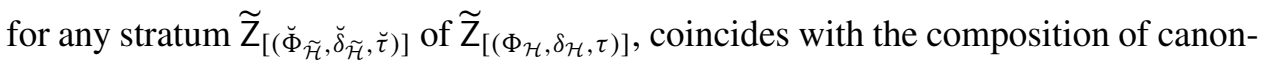

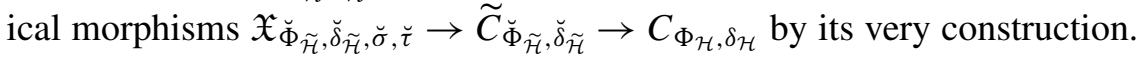

Since $f^{\text {tor }}$ is étale locally given by morphisms between toric schemes equivariant under (surjective) morphisms between tori, to determine if $f^{\text {tor }}$ is equidimensional (cf. [Faltings and Chai 1990, Chapter VI, Definition 1.3 and Remark 1.4]), it suffices to determine if the relative dimension of each of the induced (smooth) morphism $\widetilde{Z}_{\left[\left(\breve{\Phi}_{\tilde{\mathcal{H}}}, \breve{\delta}_{\widetilde{\mathcal{H}}}, \breve{\tau}^{\prime}\right]\right.} \rightarrow \mathrm{Z}_{\left[\left(\Phi_{\mathcal{H}}, \delta_{\mathcal{H}}, \tau\right)\right]}$ between strata is at most $\operatorname{dim}_{\mathrm{M}_{\mathcal{H}}}(\mathrm{N})$, the relative dimension of $f: \mathrm{N} \rightarrow \mathrm{M}_{\mathcal{H}}$.

By abuse of language, we define the $\mathbb{R}$-dimension of a cone to be the $\mathbb{R}$-dimension of its $\mathbb{R}$-span. Then the codimension of $N=\widetilde{Z}_{\left[\left(\widetilde{\Phi}_{\tilde{\mathcal{H}}}, \tilde{\delta}_{\widetilde{\mathcal{H}}}, \widetilde{\sigma}\right)\right]}$ in $\tilde{\mathrm{M}}_{\widetilde{\mathcal{H}}}^{\text {tor }}$ is $\operatorname{dim}_{\mathbb{R}}(\widetilde{\sigma})=$ $\operatorname{dim}_{\mathbb{R}}\left(\left(\mathbf{S}_{\widetilde{\Phi}}\right)_{\widetilde{\mathcal{H}}}^{\vee}\right)$ because $\widetilde{\sigma}$ is top-dimensional. The codimension of

$$
\widetilde{Z}_{\left[\left(\breve{\Phi}_{\tilde{\mathcal{H}}}, \breve{\mathcal{H}}_{\widetilde{\mathcal{H}}}, \breve{\tau}\right)\right]} \cong\left(\widetilde{\Xi}_{\breve{\Phi}_{\widetilde{\mathcal{H}}}, \breve{\delta}_{\widetilde{\mathcal{H}}}}\right)_{\breve{\tau}}
$$

in $\widetilde{\mathrm{M}}_{\widetilde{\mathcal{H}}}^{\text {tor }}$ is equal to $\operatorname{dim}_{\mathbb{R}}(\breve{\tau})$. Therefore, the codimension of $\widetilde{\mathrm{Z}}_{\left[\left(\breve{\Phi}_{\widetilde{\mathcal{H}}}, \breve{\delta}_{\widetilde{\mathcal{H}}}, \breve{\tau}\right)\right]}$ in $\mathrm{N}^{\text {tor }}$ is equal to $\operatorname{dim}_{\mathbb{R}}(\breve{\tau})-\operatorname{dim}_{\mathbb{R}}(\widetilde{\sigma})=\operatorname{dim}_{\mathbb{R}}(\breve{\tau})-\operatorname{dim}_{\mathbb{R}}\left(\left(\mathbf{S}_{\widetilde{\Phi}_{\widetilde{\mathcal{H}}}}\right)_{\mathbb{R}}^{\vee}\right)$. On the other hand, the codimension of $Z_{\left[\left(\Phi_{\mathcal{H}}, \delta_{\mathcal{H}}, \tau\right)\right]} \cong\left(\Xi_{\Phi_{\mathcal{H}}, \delta_{\mathcal{H}}}\right)_{\tau}$ in $\mathrm{M}_{\mathcal{H}}^{\text {tor }}$ is $\operatorname{dim}_{\mathbb{R}}(\tau)$. Hence we have

$$
\begin{aligned}
& \operatorname{dim}_{Z_{\left[\left(\Phi_{\mathcal{H}}, \delta \mathcal{H}, \tau\right)\right]}}\left(\widetilde{Z}_{\left[\left(\breve{\Phi}_{\tilde{\mathcal{H}}}, \breve{\delta}_{\tilde{\mathcal{H}}}, \breve{\tau}\right)\right]}\right) \\
& =\operatorname{dim}_{M_{\mathcal{H}}}(\mathrm{N})-\left(\operatorname{dim}_{\mathbb{R}}(\breve{\tau})-\operatorname{dim}_{\mathbb{R}}\left(\left(\mathbf{S}_{\widetilde{\Phi}_{\tilde{\mathcal{H}}}}\right)_{\mathbb{R}}^{\vee}\right)\right)+\operatorname{dim}_{\mathbb{R}}(\tau) .
\end{aligned}
$$

Let $\tau^{\prime}$ denote the image of $\breve{\tau}$ in $\left(\mathbf{S}_{\Phi_{\mathcal{H}}}\right)_{\mathbb{R}}^{\vee}$. By assumption on $\breve{\tau}$, we have $\tau^{\prime} \subset \tau$. If $\tau^{\prime}=\tau$, then

$$
\operatorname{dim}_{\mathbb{R}}(\tau)=\operatorname{dim}_{\mathbb{R}}\left(\tau^{\prime}\right) \leq \operatorname{dim}_{\mathbb{R}}(\tilde{\tau})-\operatorname{dim}_{\mathbb{R}}\left(\left(\mathbf{S}_{\widetilde{\Phi}_{\tilde{\mathcal{H}}}}\right)_{\mathbb{R}}^{\vee}\right),
$$

and hence (3.16) implies

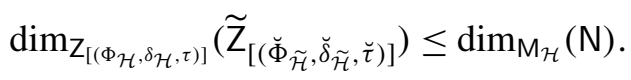

(If this is true for all $\widetilde{Z}_{\left[\left(\breve{\Phi}_{\tilde{\mathcal{H}}}, \breve{\delta}_{\widetilde{\mathcal{H}}}, \breve{\tau}\right)\right]}$, then $f^{\text {tor }}$ is equidimensional.) On the other hand, suppose $\tau^{\prime} \subsetneq \tau$. Then there exists a face of $\tau^{\prime \prime}$ of $\tau^{\prime}$ such that $\tau^{\prime \prime} \subset \tau$ and $\operatorname{dim}_{\mathbb{R}}\left(\tau^{\prime \prime}\right)<\operatorname{dim}_{\mathbb{R}}(\tau)$. Note that $\tau^{\prime \prime}$ is the image of at least one face of $\breve{\tau}$ satisfying the three conditions in the first paragraph of this section. By dropping redundant basis vectors, we may assume moreover that this face $\breve{\tau}^{\prime \prime}$ of $\breve{\tau}$ satisfies $\operatorname{dim}_{\mathbb{R}}\left(\tau^{\prime \prime}\right)=\operatorname{dim}_{\mathbb{R}}\left(\breve{\tau}^{\prime \prime}\right)-\operatorname{dim}_{\mathbb{R}}\left(\left(\mathbf{S}_{\widetilde{\Phi}_{\widetilde{\mathcal{H}}}}\right)_{\mathbb{R}}^{\vee}\right)$. Then we have

$$
\operatorname{dim}_{\mathbb{R}}(\tau)>\operatorname{dim}_{\mathbb{R}}\left(\tau^{\prime \prime}\right)=\operatorname{dim}_{\mathbb{R}}\left(\breve{\tau}^{\prime \prime}\right)-\operatorname{dim}_{\mathbb{R}}\left(\left(\mathbf{S}_{\widetilde{\Phi}_{\widetilde{\mathcal{H}}}}\right)_{\mathbb{R}}^{\vee}\right),
$$

and hence (3.16) implies

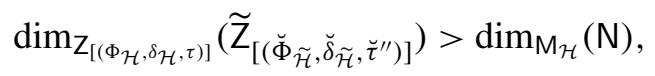

which means $f^{\text {tor }}$ cannot be equidimensional. 
This motivates the following strengthening of Condition 3.8:

Condition 3.17 (cf. [Faltings and Chai 1990, Chapter VI, Definition 1.3]). For each $\left(\breve{\Phi}_{\tilde{\mathcal{H}}}, \breve{\delta}_{\tilde{\mathcal{H}}}, \breve{\tau}\right)$ such that $\mathrm{Z}_{\left[\left(\breve{\Phi}_{\tilde{\mathcal{H}}}, \breve{\delta}_{\tilde{\mathcal{H}}}, \breve{\tau}\right)\right]}$ is (a stratum) in $\mathrm{N}^{\text {tor }}$, the image of $\breve{\tau} \subset \mathbf{P}_{\Phi_{\mathcal{H}}}^{+}$ under the (canonical) second morphism in (3.7) is exactly some cone $\tau \subset \mathbf{P}_{\Phi_{\mathcal{H}}}^{+}$in the cone decomposition $\Sigma_{\Phi_{\mathcal{H}}}$.

Proposition 3.18. The morphism $f^{\text {tor }}: \mathrm{N}^{\text {tor }} \rightarrow \mathrm{M}_{\mathcal{H}}^{\text {tor }}$ is equidimensional (with relative dimension equal to the one of $f: \mathrm{N} \rightarrow \mathrm{M}_{\mathcal{H}}$ ), and hence flat, if and only if Condition 3.17 is satisfied, if and only if $f^{\text {tor }}$ is log integral (see [Kato 1989, Definition 4.3]).

Proof. The equivalence between Condition 3.17 and equidimensionality has been explained above. Since both $\mathrm{N}^{\text {tor }}$ and $\mathrm{M}_{\mathcal{H}}^{\text {tor }}$ are regular (because they are smooth over $\left.\mathrm{S}_{0}=\operatorname{Spec}\left(\mathcal{O}_{F_{0},(\square)}\right)\right)$, the equidimensionality and flatness of $f^{\text {tor }}$ are equivalent by [EGA IV $31966,15.4 .2$ b) $\Leftrightarrow \mathrm{e}^{\prime}$ )]. By [Kato 1989, Proposition 4.1(2)], the log integrality of $f^{\text {tor }}$ is equivalent to the flatness of each of the canonical morphisms $\mathbb{Z}\left[\tau^{\vee}\right] \hookrightarrow \mathbb{Z}\left[\breve{\tau}^{\vee}\right]$ (defined when $Z_{\left[\left(\breve{\Phi}_{\tilde{\mathcal{H}}}, \breve{\delta}_{\tilde{\mathcal{H}}}, \breve{\tau}\right)\right]}$ is mapped to $\mathrm{Z}_{\left[\left(\Phi_{\mathcal{H}}, \delta_{\mathcal{H}}, \tau\right)\right]}$ ), which is equivalent to the equidimensionality of any such morphism (by the smoothness of $\mathbb{Z}\left[\tau^{\vee}\right]$ and $\mathbb{Z}\left[\breve{\tau}^{\vee}\right]$ over $\mathbb{Z}$, and by [EGA $\left.\left.\left.\operatorname{IV}_{3} 1966,15.4 .2 \mathrm{~b}\right) \Leftrightarrow \mathrm{e}^{\prime}\right)\right]$ again), which is equivalent to Condition 3.17 by the same (dimension comparison) argument.

Proposition 3.19 (cf. [Faltings and Chai 1990, Chapter VI, Remark 1.4]). Condition 3.17 can be achieved by replacing both the cone decompositions $\widetilde{\Sigma}$ and $\Sigma$ with some refinements.

Proof. Instead of taking refinements of $\widetilde{\Sigma}$ and $\Sigma$ separately, we consider the morphism $\mathbf{P}_{\breve{\Phi}_{\widetilde{\mathcal{H}}}} \rightarrow \mathbf{P}_{\Phi_{\mathcal{H}}}$ in (3.7) and consider the graph of $\widetilde{\Sigma}$. More precisely, using the canonical morphisms $X \hookrightarrow \breve{X}$ and $Y \hookrightarrow \breve{Y}$ compatible with $\phi$ and $\breve{\phi}$, we obtain canonical morphisms $X^{\prime}:=\breve{X} \oplus X \rightarrow \breve{X}$ and $Y^{\prime}:=\breve{Y} \oplus Y \rightarrow \breve{Y}$ compatible with $\phi^{\prime}:=\breve{\phi} \oplus \phi$

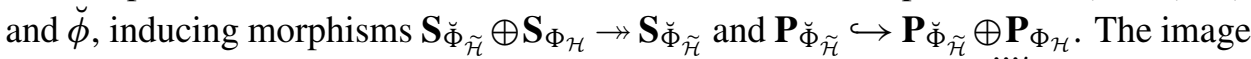
of this latter morphism is the graph of $\mathbf{P}_{\check{\Phi}_{\tilde{\mathcal{H}}}} \rightarrow \mathbf{P}_{\Phi_{\mathcal{H}}}$. Let us define $\dddot{\mathbf{S}}^{\prime}$ by $X^{\prime}, Y^{\prime}$, and $\phi^{\prime}$ as in (1.21), and let $\mathbf{S}^{\prime}$ be its free quotient. Define $\mathbf{P}^{\prime}$ accordingly as the subset of $\left(\mathbf{S}^{\prime}\right)_{\mathbb{R}}^{\vee}$ consisting of positive semidefinite pairings with admissible radicals, containing the graph of $\mathbf{P}_{\breve{\Phi}_{\tilde{\mathcal{H}}}} \rightarrow \mathbf{P}_{\Phi_{\mathcal{H}}}$ canonically as an admissible boundary component (cf. Definition 1.28). The cone decomposition $\widetilde{\Sigma}_{\breve{\Phi}_{\tilde{\mathcal{H}}}}$ defines a cone decomposition on this graph, which might fail to be projective or smooth with respect to the structure of the ambient space. But we can find a projective smooth cone decomposition of $\mathbf{P}^{\prime}$, admissible with respect to the actions of all elements in $\mathrm{GL}_{\mathcal{O}}\left(X^{\prime}\right) \times \mathrm{GL}_{\mathcal{O}}\left(Y^{\prime}\right)$ respecting $\phi^{\prime}$, such that its restriction to the graph refine the cone decomposition defined by $\widetilde{\Sigma}_{\breve{\Phi}_{\tilde{\mathcal{H}}}}$. Thus we obtain a simultaneous smooth projective refinement of $\widetilde{\Sigma}_{\breve{\Phi}_{\widetilde{\mathcal{H}}}}$ and $\Sigma_{\Phi_{\mathcal{H}}}$, such that image of cones in $\widetilde{\Sigma}_{\breve{\Phi}_{\widetilde{\mathcal{H}}}}$ under $\mathbf{P}_{\overleftarrow{\Phi}_{\widetilde{\mathcal{H}}}} \rightarrow \mathbf{P}_{\Phi_{\mathcal{H}}}$ are cones in $\Sigma_{\Phi_{\mathcal{H}}}$. Since this construction is compatible with surjections between different 
choices of $\breve{\Phi}_{\widetilde{\mathcal{H}}}$ and $\Phi_{\mathcal{H}}$, we can conclude by induction on magnitude of cusp labels $\left(\Phi_{\mathcal{H}}, \delta_{\mathcal{H}}\right)$ as in the proofs of [Lan 2008, Propositions 6.3.3.3 and 7.3.1.5].

Remark 3.20. We will not need Propositions 3.18 and 3.19 in what follows. We supply them here because knowing flatness or log integrality of $f^{\text {tor }}$ is useful in many applications.

3E. Hecke actions. The aim of this subsection is to explain the proof of statements (4) and (5) of Theorem 2.15, with (4c) and (5c) conditional on (3b) and (3c) of Theorem 2.15. These statements might seem elaborate, but they are self-explanatory and based on the following simple idea: Since $N$ and $N^{\text {tor }}$ are constructed using the toroidal compactifications of $\widetilde{\mathrm{M}}_{\tilde{\mathcal{H}}}$, we can use the Hecke actions on $\widetilde{\mathrm{M}}_{\widetilde{\mathcal{H}}}$ and their (compatible) extensions to toroidal compactifications provided by [Lan 2008, Proposition 6.4.3.4 in the revision].

Let $g_{h}, \mathcal{H}^{\prime}, \Sigma^{\prime}, g_{l}$, and $Q^{\prime}$ be as in (4) and (5) of Theorem 2.15. (For proving (4) and (5) of Theorem 2.15, we may assume in what follows either $g_{h}=1$ or $g_{l}=1$, although the theory works in a more general context.) Using the splitting $\tilde{\delta}$ of $\widetilde{z}$, we obtain an element $\tilde{g}$ in $\widetilde{\mathrm{P}} \widetilde{\mathrm{Z}}\left(\mathbb{A}^{\infty, \square}\right)$ such that $\mathrm{Gr}_{-1}^{\widetilde{Z}}(\tilde{g})=g_{h}$, and such that $\operatorname{Gr}_{0}^{\widetilde{Z}}(\tilde{g})$ is identified with $g_{l}^{-1}$ under $\widetilde{\varphi}_{0}: \mathrm{Gr}_{0}^{\widetilde{Z}} \stackrel{\sim}{\rightarrow} Q_{0} \otimes_{\mathbb{Z}} \hat{\mathbb{Z}}^{\square} \cong Q \otimes_{\mathbb{Z}} \hat{\mathbb{Z}}^{\square}$. (See Section 3A.) Let $\tilde{\mathcal{H}}^{\prime}$ be a (necessarily neat) subgroup of $\widetilde{\mathrm{G}}\left(\hat{\mathbb{Z}}^{\square}\right)$ such that $\tilde{g}^{-1} \tilde{\mathcal{H}}^{\prime} \tilde{g} \subset \tilde{\mathcal{H}}$, and such that $\mathcal{H}^{\prime}=\mathrm{Gr}_{-1}^{\widetilde{z}}\left(\widetilde{\mathcal{H}}^{\prime} \cap \mathrm{P}_{\widetilde{Z}}\left(\hat{\mathbb{Z}}^{\square}\right)\right)$. By [Lan 2008, Proposition 6.4.3.4 in the revision], there exist some choices of $\widetilde{\Sigma}^{\prime}$ such that the canonical morphism $[\tilde{g}]: \widetilde{M}_{\widetilde{\mathcal{H}}^{\prime}} \rightarrow \widetilde{\mathrm{M}}_{\widetilde{\mathcal{H}}}$ extends canonically to $[\tilde{g}]^{\text {tor }}: \widetilde{M}_{\widetilde{\mathcal{H}}^{\prime}, \widetilde{\Sigma}^{\prime}}^{\text {tor }} \rightarrow \widetilde{\mathrm{M}}_{\widetilde{\mathcal{H}}, \widetilde{\Sigma}}^{\text {tor }}$. By replacing $\widetilde{\Sigma}^{\prime}$ with a refinement such that it satisfies Condition 3.8 (with $\Sigma^{\prime}$ and) with some choice of $\widetilde{\sigma}^{\prime}$, and such that

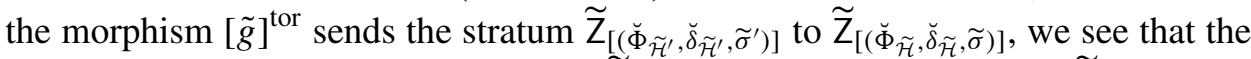

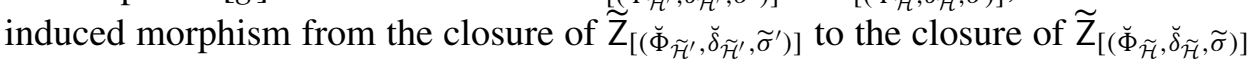
gives the existences of the morphisms $\left[g_{h}\right]_{\kappa^{\prime}, \kappa},\left[g_{h}\right]_{\kappa^{\prime}, \kappa}^{\text {tor }},\left[g_{l}\right]_{\kappa^{\prime}, \kappa}^{*}$, and $\left(\left[g_{l}\right]_{\kappa^{\prime}, \kappa}^{*}\right)^{\text {tor }}$ as in (4a), (4b), (5a), and (5b) of Theorem 2.15, where $\kappa^{\prime}=\left(\widetilde{\mathcal{H}}^{\prime}, \widetilde{\Sigma}^{\prime}, \widetilde{\sigma}^{\prime}\right)$ lies in $\mathbf{K}_{Q^{\prime}, \mathcal{H}^{\prime}, \Sigma^{\prime}}$, except that (2.24) and (2.26) still have to be explained.

As in the case of showing $R^{i}\left(f_{\kappa^{\prime}, K}^{\text {tor }}\right)_{*} \mathrm{O}_{\mathrm{N}_{\kappa^{\prime}}}=0$ for $i>0$ in Section $3 \mathrm{~A}$, since the morphisms $\left[g_{h}\right]_{\kappa^{\prime}, \kappa}^{\text {tor }}$ and $\left(\left[g_{l}\right]_{\kappa^{\prime}, \kappa}^{*}\right)^{\text {tor }}$ are étale locally given by equivariant morphisms between toric schemes, we have (by [Kempf et al. 1973, Chapter I, §3]) $\left.R^{i}\left(\left[g_{h}\right]_{\kappa^{\prime}, \kappa}^{\text {tor }}\right)_{*}\left(\mathcal{O}_{\left(\mathrm{N}_{\kappa^{\prime}}^{\prime}\right)}\right)^{\text {tor }}\right)=0$ and $R^{i}\left(\left[g_{l}\right]_{\kappa^{\prime}, \kappa}^{*}\right)_{*}^{\text {tor }}\left(\mathcal{O}_{\left(\mathrm{N}_{\kappa^{\prime}}^{\prime}\right)^{\text {tor }}}\right)=0$ for $i>0$, which are (2.24) and (2.26) of Theorem 2.15.

The remaining statements in (4c) and (5c) of Theorem 2.15 now follow if we assume statements (3b) and (3c) of Theorem 2.15. (See the end of Section 5, p. 957.)

\section{Calculation of formal cohomology}

Throughout this section, unless otherwise specified, we fix the choice of an arbitrary (locally closed) stratum $\mathrm{Z}_{\left[\left(\Phi_{\mathcal{H}}, \delta_{\mathcal{H}}, \tau\right)\right]}$ of $\mathrm{M}_{\mathcal{H}}^{\text {tor }}$. The aim of this section is to calculate 
the relative cohomology of the pullback of the structure morphism $f^{\text {tor }}$ to the formal completion $\left(\mathrm{M}_{\mathcal{H}}^{\text {tor }}\right)_{\mathrm{Z}_{\left[\left(\Phi_{\mathcal{H}}, \delta_{\mathcal{H}}, \tau\right)\right.}}$. (See (5) of Theorem 1.41 for a description of this formal completion. See also the first paragraph of Section 3D for a description of the formal completion $\left(\mathrm{N}^{\text {tor }}\right)_{\widetilde{\mathrm{Z}}_{\left[\left(\Phi_{\mathcal{H}}, \delta_{\mathcal{H}}, \tau\right)\right]}}$ of $\mathrm{N}^{\text {tor }}$ along $\widetilde{\mathrm{Z}}_{\left[\left(\Phi_{\mathcal{H}}, \delta_{\mathcal{H}}, \tau\right)\right]}=\left(f^{\text {tor }}\right)^{-1}\left(\mathrm{Z}_{\left[\left(\Phi_{\mathcal{H}}, \delta_{\mathcal{H}}, \tau\right)\right]}\right)$.)

4A. Formal fibers of $\boldsymbol{f}^{\text {tor. }}$. Let $\Gamma_{\breve{\Phi}_{\tilde{\mathcal{H}}}, \tau}$ be the subgroup of elements in $\Gamma_{\breve{\Phi}_{\tilde{\mathcal{H}}}}$ stabilizing (both) $X$ and $Y$ and inducing an element in $\Gamma_{\Phi_{\mathcal{H}}, \tau}$ (the subgroup of $\Gamma_{\Phi_{\mathcal{H}}}$ formed by elements mapping $\tau$ to itself). Since we have tacitly assumed that $\Gamma_{\Phi_{\mathcal{H}}, \tau}$ is trivial by Condition 1.29 and [Lan 2008, Lemma 6.2.5.27 in the revision], $\Gamma_{\breve{\Phi}_{\tilde{\mathcal{H}}}, \tau}$ is also the subgroup of elements in $\Gamma_{\widetilde{\Phi}} \breve{\Phi}_{\widetilde{\mathcal{H}}}$ fixing (both) $X$ and $Y$. Let $\Gamma_{\widetilde{\Phi}} \widetilde{\Phi}_{\widetilde{\mathcal{H}}}, \Phi_{\mathcal{H}}$ be the subgroup of elements in $\operatorname{Hom}_{\mathcal{O}}(\widetilde{X}, X)$ sending $\widetilde{\phi}(\widetilde{Y})$ to $\phi(Y)$ that are compatible with $\widetilde{\varphi}_{-2, \tilde{\mathcal{H}}}$, $\tilde{\varphi}_{0, \tilde{\mathcal{H}}}, \varphi_{-2, \mathcal{H}}$, and $\varphi_{0, \mathcal{H}}$. Note that these compatibility conditions imply that the subgroup $\Gamma_{\widetilde{\Phi}_{\tilde{\mathcal{H}}}, \Phi_{\mathcal{H}}}$ has index prime to $\square$ in $\operatorname{Hom}_{\mathcal{O}}(\tilde{X}, X)$. The two surjections

$$
s_{\breve{X}}: \breve{X} \rightarrow \widetilde{X} \quad \text { and } \quad s_{\breve{Y}}: \breve{Y} \rightarrow \widetilde{Y}
$$

identify $\Gamma_{\widetilde{\Phi}_{\tilde{\mathcal{H}}}, \Phi_{\mathcal{H}}}$ as a subgroup of $\Gamma_{\breve{\Phi}_{\tilde{\mathcal{H}}}, \tau}$. (More precisely, any $t \in \Gamma_{\widetilde{\Phi}_{\tilde{\mathcal{H}}}}, \Phi_{\mathcal{H}}$ defines a translation action $x \mapsto x+t\left(s_{\breve{X}}(x)\right)$ on $\breve{X}$, inducing compatibly a translation action on $\breve{Y}$, and hence defining an element in $\Gamma_{\Phi_{\tilde{\mathcal{H}}}, \tau}$ fixing both $X$ and $Y$.)

Since $\Gamma_{\widetilde{\Phi}_{\widetilde{\mathcal{H}}}, \Phi_{\mathcal{H}}}$ does not modify $s_{\breve{X}}$ and $s_{\breve{Y}}$, it does not modify the first morphism in (3.7). Therefore, if we denote the image of $\widetilde{\sigma}$ in $\mathbf{P}_{\breve{\Phi}_{\tilde{\mathcal{H}}}}$ by $\breve{\sigma}$, then $\Gamma \widetilde{\Phi}_{\widetilde{\mathcal{H}}}, \Phi_{\mathcal{H}}$ maps $\breve{\sigma}$ to itself. On the other hand, by Condition 1.29 (and Lemma 3.1), if a cone $\breve{\tau} \subset \mathbf{P}_{\breve{\Phi}_{\widetilde{\mathcal{H}}}^{+}}^{+}$in $\Sigma_{\breve{\Phi}_{\tilde{\mathcal{H}}}}$ has a face that is a $\Gamma_{\breve{\Phi}_{\tilde{\mathcal{H}}}, \tau}$-translation of $\breve{\sigma}$, then it cannot have a different face that is also a $\Gamma_{\breve{\Phi}_{\tilde{\mathcal{H}}}, \tau}$-translation of $\breve{\sigma}$. Let us denote by $\Sigma_{\breve{\Phi}_{\tilde{\mathcal{H}}}, \breve{\sigma}, \tau}$ the subset of $\Sigma_{\breve{\Phi}_{\widetilde{\mathcal{H}}}}$ consisting of cones $\breve{\tau}$ satisfying the following conditions (cf. similar conditions in the first paragraph of Section 3D):

(1) $\breve{\tau} \subset \mathbf{P}_{\Phi_{\tilde{\mathcal{H}}}}^{+}$.

(2) $\breve{\tau}$ has $\breve{\sigma}$ as a face.

(3) The image of $\breve{\tau}$ under the (canonical) second morphism in (3.7) is contained in $\tau \subset \mathbf{P}_{\Phi_{\mathcal{H}}}^{+}$.

Then, to obtain a complete list of representatives of the cusp labels $\left[\left(\breve{\Phi}_{\widetilde{\mathcal{H}}}, \breve{\delta}_{\widetilde{\mathcal{H}}}, \breve{\tau}\right)\right]$ parametrizing the strata of $\widetilde{Z}_{\left[\left(\Phi_{\mathcal{H}}, \delta_{\mathcal{H}}, \tau\right)\right]}$, it suffices to take representatives of $\Sigma_{\overleftarrow{\Phi}_{\widetilde{\mathcal{H}}}, \breve{\sigma}, \tau}$ modulo the action of $\Gamma_{\widetilde{\Phi}_{\tilde{\mathcal{H}}}}, \Phi_{\mathcal{H}}$. (That is, we do not have to consider $\Gamma_{\widetilde{\Phi}_{\tilde{\mathcal{H}}}}, \Phi_{\mathcal{H}}$-translates of $\breve{\sigma}$.)

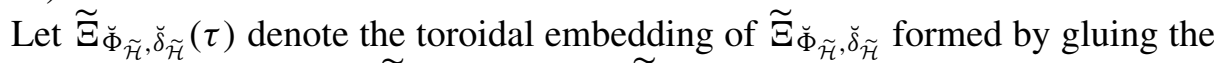

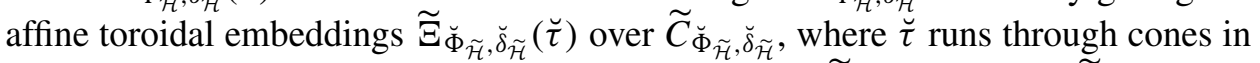

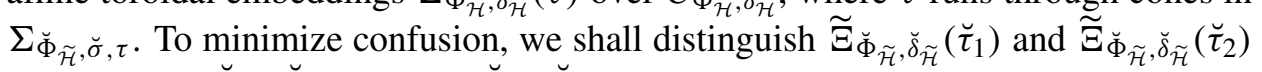
even when $\left[\left(\breve{\Phi}_{\widetilde{\mathcal{H}}}, \breve{\delta}_{\widetilde{\mathcal{H}}}, \breve{\tau}_{1}\right)\right]=\left[\left(\breve{\Phi}_{\widetilde{\mathcal{H}}}, \breve{\delta}_{\widetilde{\mathcal{H}}}, \breve{\tau}_{2}\right)\right]$. For each $\breve{\tau}$ as above (having $\breve{\sigma}$ as a

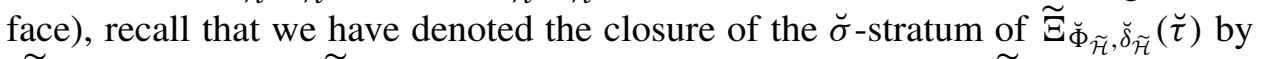
$\left(\widetilde{\Xi}_{\Phi_{\tilde{\mathcal{H}}}, \breve{\delta}_{\tilde{\mathcal{H}}}}\right)_{\breve{\sigma}}(\breve{\tau})$. Let $\left(\widetilde{\Xi}_{\Phi_{\tilde{\mathcal{H}}}, \breve{\delta}_{\widetilde{\mathcal{H}}}}\right)_{\breve{\sigma}}(\tau)$ denote the union of all such $\left(\widetilde{\Xi}_{\Phi_{\tilde{\mathcal{H}}}, \breve{\delta}_{\tilde{\mathcal{H}}}}\right)_{\breve{\sigma}}(\breve{\tau})$, let 


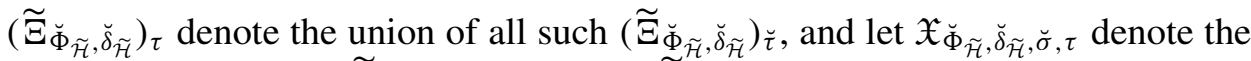
formal completion of $\left(\widetilde{\Xi}_{\Phi_{\tilde{\mathcal{H}}}}, \breve{\delta}_{\widetilde{\mathcal{H}}}\right) \breve{\sigma}(\tau)$ along $\left(\widetilde{\Xi}_{\Phi_{\tilde{\mathcal{H}}}}, \breve{\delta}_{\widetilde{\mathcal{H}}}\right)_{\tau}$.

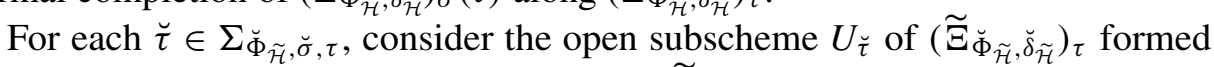

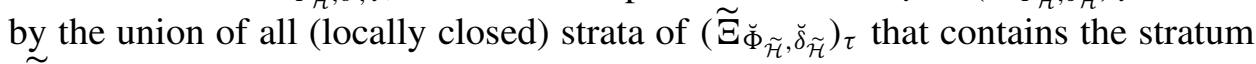

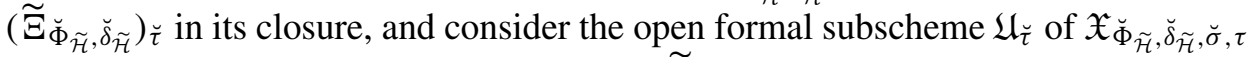

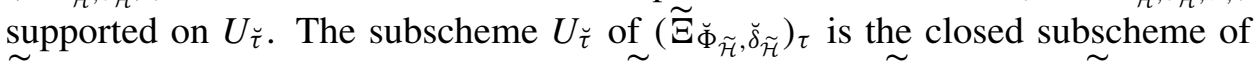

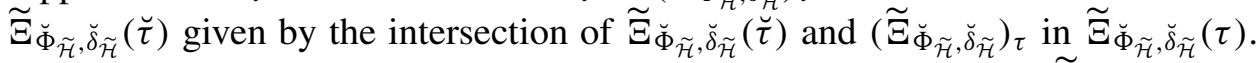

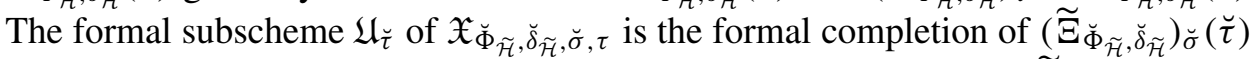

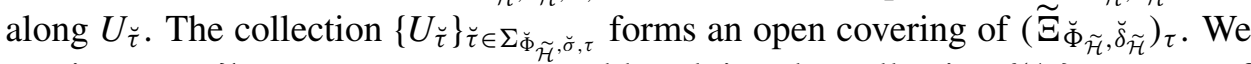

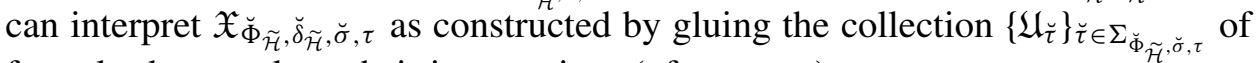
formal schemes along their intersections (of supports).

Explicitly, let us denote by $\breve{\tau}_{\breve{\sigma}}^{\vee}$ the intersection of $\left(\breve{\tau}^{\prime}\right)_{0}^{\vee}$ for $\breve{\tau}^{\prime}$ running through faces of $\breve{\tau}$ in $\Sigma_{\breve{\Phi}}, \breve{\mathcal{H}}, \tau$ (including $\breve{\tau}$ itself). Then we have the canonical isomorphism

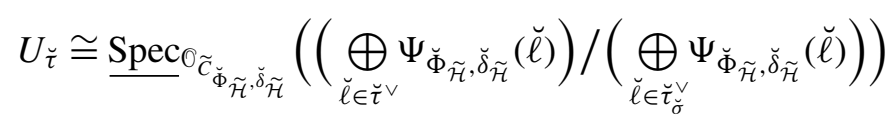

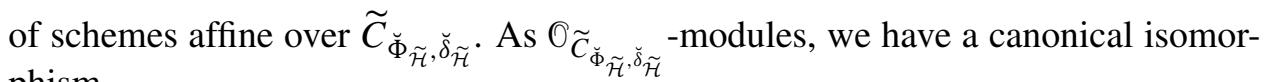
phism

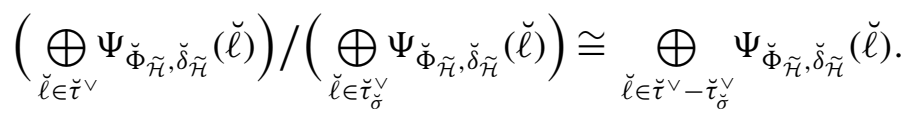

If we equip $\breve{\tau}^{\vee}-\breve{\tau}_{\breve{\sigma}}^{\vee}$ with the semigroup structure induced by the canonical bi-

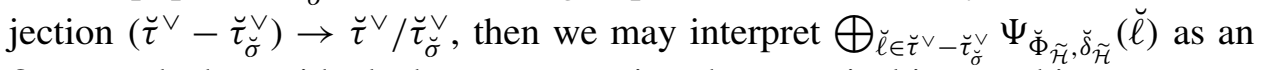
$0 \widetilde{C}_{\breve{\Phi}_{\tilde{\mathcal{H}}}, \breve{\mathscr{\delta}}_{\breve{\mathcal{H}}}}$-algebra, with algebra structure given by canonical isomorphisms

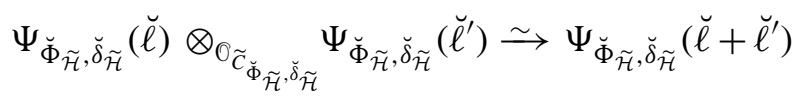

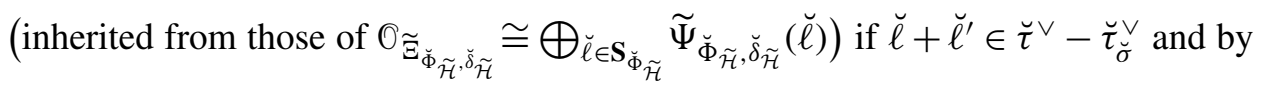

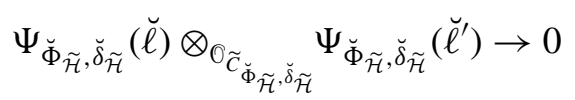

otherwise. Then we have a canonical isomorphism

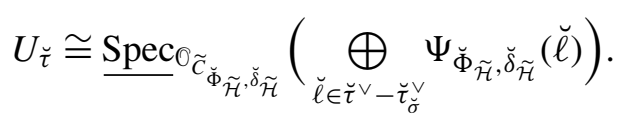

By definition, we have

$$
\breve{\tau}^{\vee}-\breve{\tau}_{\breve{\sigma}}^{\vee}=\left(\bigcup_{\substack{\breve{\tau}^{\prime} \text { face of } \breve{\tau} \\ \text { in } \Sigma_{\breve{\Phi}_{\tilde{\mathcal{H}}}, \breve{\sigma}, \tau}}}\left(\left(\breve{\tau}^{\prime}\right)^{\perp} \cap \breve{\tau}^{\vee}\right)\right) \subset \breve{\sigma}^{\perp} \cap \breve{\tau}^{\vee} .
$$


The formal scheme $\mathfrak{U}_{\bar{\tau}}$, being the formal completion of

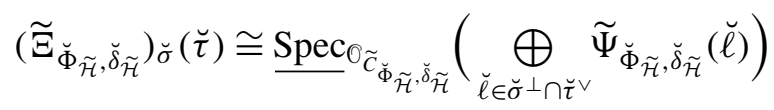

along $U_{\breve{\tau}}$, can be canonically identified with the relative formal spectrum of the $0 \widetilde{C}_{\check{\Phi}_{\tilde{\mathcal{H}}}, \breve{\mathcal{\delta}}_{\tilde{\mathcal{H}}}}$-algebra $\widehat{\bigoplus}_{\breve{\ell} \in \breve{\sigma}^{\perp} \cap \breve{\tau}^{\vee}} \Psi_{\breve{\Phi}_{\tilde{\mathcal{H}}}, \breve{\delta}_{\tilde{\mathcal{H}}}}(\breve{\ell})$ over $\widetilde{C}_{\breve{\Phi}_{\widetilde{\mathcal{H}}}, \breve{\delta}_{\tilde{\mathcal{H}}}}$, where $\widehat{\bigoplus}$ denotes the com-

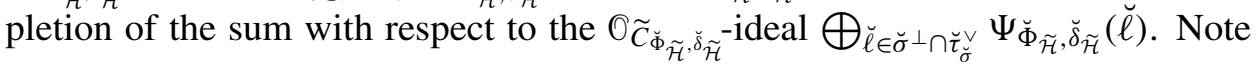
that all the above canonical isomorphisms correspond to canonical morphisms

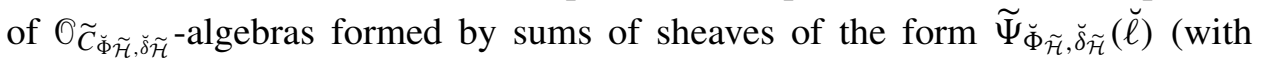

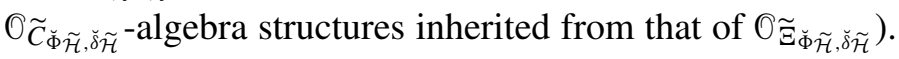

The descriptions above imply the following simple but important facts:

Lemma 4.1. Suppose $\breve{\tau}$ and $\breve{\tau}^{\prime}$ are two cones in $\Sigma_{\breve{\Phi}_{\widetilde{\mathcal{H}}}, \breve{\sigma}, \tau}$ such that $\breve{\tau}^{\prime}$ is a face of $\breve{\tau}$.

(1) We have a canonical open immersion $\mathfrak{U}_{\breve{\tau}^{\prime}} \hookrightarrow \mathfrak{U}_{\bar{\tau}}$ (resp. $U_{\breve{\tau}^{\prime}} \hookrightarrow U_{\breve{\tau}}$ ) offormal subschemes of $\mathfrak{X}_{\breve{\Phi}_{\tilde{\mathcal{H}}}, \breve{\delta}_{\tilde{\mathcal{H}}}, \breve{\sigma}, \tau}$.

(2) The canonical restriction morphism from $\mathfrak{U}_{\bar{\tau}}$ to $\mathfrak{U}_{\bar{\tau}^{\prime}}$ corresponds to the canonical morphism

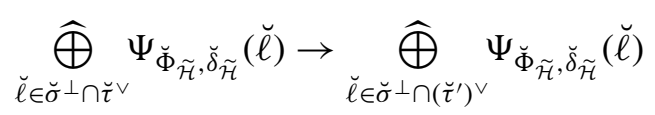

of $\mathcal{O} \widetilde{C}_{\check{\Phi}_{\tilde{\mathcal{H}}}, \breve{s}_{\mathcal{H}}}$-algebras, where the two symbols $\widehat{\bigoplus}$ denote completions of the sums

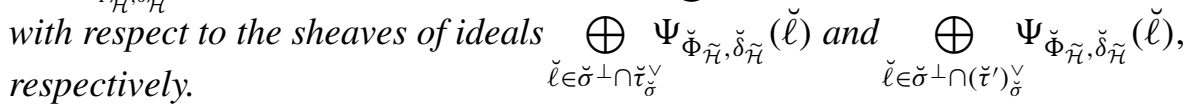

(3) The canonical restriction morphism from $U_{\breve{\tau}}$ to $U_{\breve{\tau}^{\prime}}$ corresponds to the canonical morphism

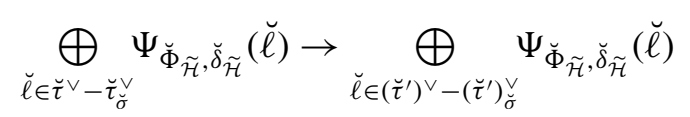

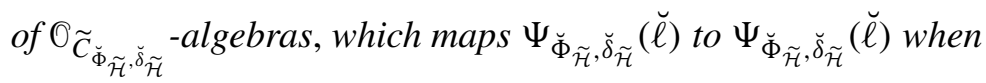

$$
\breve{\ell} \in\left(\breve{\tau}^{\vee}-\left(\breve{\tau}^{\prime}\right)_{\breve{\sigma}}^{\vee}\right)=\left(\breve{\tau}^{\vee}-\breve{\tau}_{\breve{\sigma}}^{\vee}\right) \cap\left(\left(\breve{\tau}^{\prime}\right)^{\vee}-\left(\breve{\tau}^{\prime}\right)_{\breve{\sigma}}^{\vee}\right)
$$

and to zero otherwise.

(4) The correspondences in (2) and (3) above are canonically compatible with each other.

By Condition 1.29 (and Lemma 3.1), the action of $\Gamma_{\widetilde{\Phi}_{\tilde{\mathcal{H}}}, \Phi_{\mathcal{H}}}$ induces only the trivial action on each stratum it stabilizes. Therefore, the quotient morphism

$$
\mathfrak{X}_{\breve{\Phi}_{\widetilde{\mathcal{H}}}, \breve{\delta}_{\widetilde{\mathcal{H}}}, \breve{\sigma}, \tau} \rightarrow \mathfrak{X}_{\breve{\Phi}_{\widetilde{\mathcal{H}}}, \breve{\delta}_{\tilde{\mathcal{H}}}, \breve{\sigma}, \tau} / \Gamma_{\widetilde{\Phi}_{\widetilde{\mathcal{H}}}, \Phi_{\mathcal{H}}}
$$


of formal schemes over $\mathrm{S}_{0}$ is a local isomorphism. The morphism (4.2) is not defined

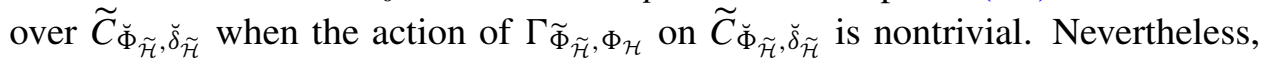
since $\Gamma_{\Phi_{\tilde{\mathcal{H}}}}, \Phi_{\mathcal{H}}$ acts trivially on $\Phi_{\mathcal{H}}$, it acts trivially on $C_{\Phi_{\mathcal{H}}, \delta_{\mathcal{H}}}$, and hence (4.2) is defined over $C_{\Phi_{\mathcal{H}}, \delta_{\mathcal{H}}}$.

Proposition 4.3. There is a canonical isomorphism

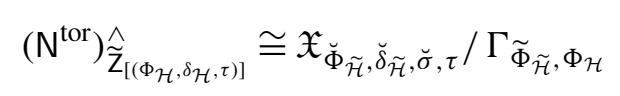

of formal schemes over $C_{\Phi_{\mathcal{H}}, \delta_{\mathcal{H}}}$, characterized by the identifications

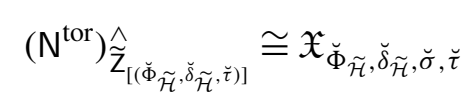

of formal schemes over $\widetilde{C}_{\breve{\Phi}_{\widetilde{\mathcal{H}}}, \breve{\delta}_{\widetilde{\mathcal{H}}}}$ (compatible with the canonical morphisms

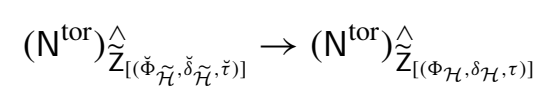

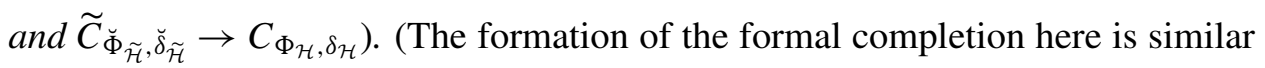
to the one in (5) of Theorem 1.41.)

Proof. Let $\breve{\tau} \in \Sigma_{\breve{\Phi}_{\widetilde{\mathcal{H}}}, \breve{\sigma}, \tau}$. Let $\widetilde{\mathfrak{U}}_{\breve{\tau}}$ denote the completion of $\widetilde{\Xi}_{\breve{\Phi}_{\tilde{\mathcal{H}}}, \breve{\delta}_{\widetilde{\mathcal{H}}}}(\breve{\tau})$ along $U_{\breve{\tau}}$, which contains $\mathfrak{U}_{\breve{\tau}}$ as a closed formal subscheme (with the same support $U_{\breve{\tau}}$ ).

Since $U_{\breve{\tau}}$ is the union of $\left(\widetilde{\Xi}_{\Phi_{\tilde{\mathcal{H}}}, \breve{\delta}_{\tilde{\mathcal{H}}}}\right)_{\breve{\tau}^{\prime}}$ with $\breve{\tau}^{\prime}$ running through faces of $\breve{\tau}$ in $\Sigma_{\overleftarrow{\Phi}_{\tilde{\mathcal{H}}}, \breve{\sigma}, \tau}$, which are cones in $\mathbf{P}_{\breve{\Phi}_{\tilde{\mathcal{H}}}^{+}}^{+}$, the tautological degeneration data over $\widetilde{\mathfrak{U}}_{\breve{\tau}}$ satisfies the positivity condition (with respect to the ideal defining $U_{\breve{\tau}}$ ), and we obtain by Mumford's construction a degenerating family $\left({ }^{\circ} \widetilde{G},{ }^{\circ} \widetilde{\lambda},{ }^{\circ} \tilde{i},{ }^{\circ} \widetilde{\alpha}_{\tilde{\mathcal{H}}}\right) \rightarrow \widetilde{\mathfrak{U}}_{\bar{\tau}}$ as in [Lan 2008, §6.2.5; especially the paragraph preceding Definition 6.2.5.17], called a Mumford family. Note that a Mumford family is defined in the sense of relative schemes, namely as a functorial assignment to each affine open formal subscheme $\operatorname{Spf}(R)$ of $\widetilde{\mathfrak{U}}_{\breve{\tau}}$ a degenerating family over $\operatorname{Spec}(R)$. Therefore (6) of Theorem 1.41 applies, and implies the existence of a canonical (strata-preserving) morphism $\widetilde{\mathfrak{U}}_{\tilde{\tau}} \rightarrow \widetilde{\mathcal{M}}_{\widetilde{\mathcal{H}}}^{\text {tor }}$ under which $\left({ }^{\circ} \widetilde{G},{ }^{\circ} \widetilde{\lambda},{ }^{\circ} \tilde{i},{ }^{\circ} \widetilde{\alpha}_{\tilde{\mathcal{H}}}\right) \rightarrow \widetilde{\mathfrak{U}}_{\tilde{\tau}}$ is the pullback of $\left(\widetilde{G}, \widetilde{\lambda}, \tilde{i}, \widetilde{\alpha}_{\widetilde{\mathcal{H}}}\right) \rightarrow \widetilde{\mathrm{M}}_{\widetilde{\mathcal{H}}}^{\text {tor }}$. Moreover, if $\breve{\tau}^{\prime} \in \Sigma_{\breve{\Phi}_{\tilde{\mathcal{H}}}, \breve{\sigma}, \tau}$, then the morphisms from $\widetilde{\mathfrak{U}}_{\bar{\tau}}$ and from $\widetilde{\mathfrak{U}}_{\bar{\tau}^{\prime}}$ to $\widetilde{M}_{\widetilde{\mathcal{H}}}^{\text {tor }}$ agree over the intersection $\widetilde{\mathfrak{U}}_{\tilde{\tau}} \cap \widetilde{\mathfrak{U}}_{\widetilde{\tau}^{\prime}}$.

By taking the closures of the $\left[\left(\widetilde{\Phi}_{\tilde{\mathcal{H}}}, \tilde{\delta}_{\tilde{\mathcal{H}}}, \widetilde{\sigma}\right)\right]$-strata (not as closed subschemes of the supports, but as closed formal subschemes, as in the second last paragraph preceding Condition 3.8), we obtain canonical morphisms $\mathfrak{U}_{\breve{\tau}} \rightarrow \mathrm{N}^{\text {tor }}$ for all $\breve{\tau}$ in $\Sigma_{\breve{\Phi}_{\tilde{\mathcal{H}}}, \breve{\sigma}, \tau}$, which patch together, cover all strata above $\left[\left(\Phi_{\mathcal{H}}, \delta_{\mathcal{H}}, \tau\right)\right]$, and define (4.4) as desired.

By (5) of Theorem 1.41, we have a canonical isomorphism

$$
\left(\mathrm{M}_{\mathcal{H}}^{\text {tor }}\right)_{\hat{Z}_{\left[\left(\Phi_{\mathcal{H}}, \delta_{\mathcal{H}}, \tau\right)\right]}} \cong \mathfrak{X}_{\Phi_{\mathcal{H}}, \delta_{\mathcal{H}}, \tau} .
$$


By the very constructions, we may and we shall identify the pullback of $f^{\text {tor }}$ to $\left(\mathrm{M}_{\mathcal{H}}^{\text {tor }}\right)_{\mathrm{Z}_{\left[\left(\Phi_{\mathcal{H}}, \delta_{\mathcal{H}}, \tau\right)\right]}}$ with the canonical morphism $\mathfrak{X}_{\breve{\Phi}_{\tilde{\mathcal{H}}}, \breve{\mathcal{F}}_{\widetilde{\mathcal{H}}}, \breve{\sigma}, \tau} / \Gamma_{\widetilde{\Phi}_{\widetilde{\mathcal{H}}}, \Phi_{\mathcal{H}}} \rightarrow \mathfrak{X}_{\Phi_{\mathcal{H}}, \delta_{\mathcal{H}}, \tau}$. By abuse of notation, we shall also denote this pullback by

$$
f^{\text {tor }}: \mathfrak{X}_{\breve{\Phi}_{\tilde{\mathcal{H}}}, \breve{\delta}_{\widetilde{\mathcal{H}}}, \breve{\sigma}, \tau} / \Gamma_{\widetilde{\Phi}_{\widetilde{\mathcal{H}}}, \Phi_{\mathcal{H}}} \rightarrow \mathfrak{X}_{\Phi_{\mathcal{H}}, \delta_{\mathcal{H}}, \tau} .
$$

For each $\breve{\tau} \in \Sigma_{\breve{\Phi}_{\tilde{\mathcal{H}}}, \breve{\sigma}, \tau}$, let $\mathfrak{U}_{[\breve{\tau}]}$ denote the image of $\mathfrak{U}_{\breve{\tau}}$ under (4.2), which is isomorphic to $\mathfrak{U}_{\tilde{\tau}}$ as a formal scheme over $C_{\Phi_{\mathcal{H}}, \delta_{\mathcal{H}}}$. By admissibility of $\Sigma_{\breve{\Phi}_{\tilde{\mathcal{H}}}}$,

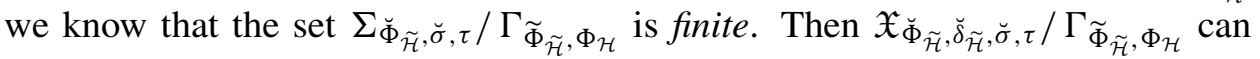

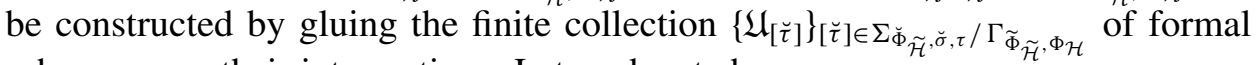
schemes over their intersections. Let us denote by

$$
f_{[\bar{\tau}]}^{\text {tor }}: \mathfrak{U}_{[\breve{\tau}]} \rightarrow \mathfrak{X}_{\Phi_{\mathcal{H}}, \delta_{\mathcal{H}}, \tau}
$$

the restriction of $f^{\text {tor }}$ to $\mathfrak{U}_{[\breve{\tau}]}$. If we choose a representative $\breve{\tau}$ of $[\breve{\tau}]$, then we can identify $f_{[\breve{\tau}]}^{\text {tor }}: \mathfrak{U}_{[\breve{\tau}]} \rightarrow \mathfrak{X}_{\Phi_{\mathcal{H}}, \delta_{\mathcal{H}}, \tau}$ with the canonical morphism $f_{\breve{\tau}}^{\text {tor }}: \mathfrak{U}_{\breve{\tau}} \rightarrow \mathfrak{X}_{\Phi_{\mathcal{H}}, \delta_{\mathcal{H}}, \tau}$ induced by the canonical morphism $\mathfrak{X}_{\breve{\Phi}_{\tilde{\mathcal{H}}}, \breve{\delta}_{\tilde{H}}, \breve{\sigma}, \tau} \rightarrow \mathfrak{X}_{\Phi_{\mathcal{H}}, \delta_{\mathcal{H}}, \tau}$. Let us denote by

$$
g_{\breve{\tau}}: \mathfrak{U}_{\bar{\tau}} \rightarrow \mathfrak{X}_{\Phi_{\mathcal{H}}, \delta_{\mathcal{H}}, \tau} \times_{C_{\Phi_{\mathcal{H}}, \delta_{\mathcal{H}}}} \widetilde{C}_{\breve{\Phi}_{\tilde{\mathcal{H}}}, \breve{\delta}_{\tilde{\mathcal{H}}}}, \quad h: \widetilde{C}_{\breve{\Phi}_{\tilde{\mathcal{H}}}, \breve{\delta}_{\tilde{\mathcal{H}}}} \rightarrow C_{\Phi_{\mathcal{H}}, \mathrm{Z}_{\mathcal{H}}},
$$

and

$$
h_{\tau}: \mathfrak{X}_{\Phi_{\mathcal{H}}, \delta_{\mathcal{H}}, \tau} \times_{C_{\Phi_{\mathcal{H}}, \delta_{\mathcal{H}}}} \widetilde{C}_{\breve{\Phi}_{\widetilde{\mathcal{H}}}, \breve{\delta}_{\widetilde{\mathcal{H}}}} \rightarrow \mathfrak{X}_{\Phi_{\mathcal{H}}, \delta_{\mathcal{H}}, \tau}
$$

the canonical morphisms. Then we have a canonical identification $f_{\breve{\tau}}^{\text {tor }}=h_{\tau} \circ g_{\breve{\tau}}$. (Note that $g_{\breve{\tau}}$ is a morphism between affine formal schemes over $\widetilde{C}_{\breve{\Phi}_{\widetilde{\mathcal{H}}}, \breve{\delta}_{\widetilde{\mathcal{H}}}}$, and that $h_{\tau}$ is the pullback of $h$ to the affine formal scheme $\mathfrak{X}_{\Phi_{\mathcal{H}}, \delta_{\mathcal{H}}, \tau}$ over $C_{\Phi_{\mathcal{H}}, \delta_{\mathcal{H}}}$.)

For simplicity, let us view $0_{\mathfrak{X}_{\Phi_{\mathcal{H}}, \delta_{\mathcal{H}}, \tau}}$ and $\mathrm{O}_{\mathrm{Z}_{\left[\left(\Phi_{\mathcal{H}}, \delta_{\mathcal{H}}, \tau\right]\right]}}$ as sheaves over $C_{\Phi_{\mathcal{H}}, \delta_{\mathcal{H}}}$, and suppress $\left(\mathfrak{X}_{\Phi_{\mathcal{H}}, \delta_{\mathcal{H}}, \tau} \rightarrow C_{\Phi_{\mathcal{H}}, \delta_{\mathcal{H}}}\right)_{*}$ and $\left(\mathrm{Z}_{\left[\left(\Phi_{\mathcal{H}}, \delta_{\mathcal{H}}, \tau\right)\right]} \rightarrow C_{\Phi_{\mathcal{H}}, \delta_{\mathcal{H}}}\right)_{*}$ from the notation. For push-forwards (to $C_{\Phi_{\mathcal{H}}, \delta_{\mathcal{H}}}$ ) of sheaves over $\mathfrak{X}_{\Phi_{\mathcal{H}}, \delta_{\mathcal{H}}, \tau}$, we shall use the notation $\widehat{\bigoplus}$ to denote the completion with respect to (the push-forward of) the ideal of definition of $\mathrm{O}_{\mathfrak{X}_{\Phi_{\mathcal{H}}, \delta_{\mathcal{H}}, \tau}}$.

Based on Lemma 4.1, we have the following important facts:

Lemma 4.6. (1) For any $\breve{\tau} \in \Sigma_{\breve{\Phi}_{\widetilde{\mathcal{H}}}, \breve{\sigma}, \tau}$, and any integer $d \geq 0$, we have the canonical isomorphisms

$$
R^{d}\left(f_{\breve{\tau}}^{\text {tor }}\right)_{*}\left(\mathcal{O}_{\mathfrak{U}_{\breve{\tau}}}\right) \cong \underset{\breve{\ell} \in \breve{\sigma}^{\perp} \cap \breve{\tau}^{\vee}}{\widehat{\bigoplus}} R^{d} h_{*}\left(\Psi_{\breve{\Phi}_{\tilde{\mathcal{H}}}, \breve{\mathcal{F}}_{\widetilde{\mathcal{H}}}}(\breve{\ell})\right)
$$

and

$$
R^{d}\left(f_{\breve{\tau}}^{\text {tor }}\right)_{*}\left(\mathcal{O}_{U_{\breve{\tau}}}\right) \cong \bigoplus_{\breve{\ell} \in \breve{\tau}^{\vee}-\breve{\tau}_{\breve{\sigma}}^{\vee}} R^{d} h_{*}\left(\Psi_{\breve{\Phi}_{\tilde{\mathcal{H}}}, \breve{\delta}_{\tilde{\mathcal{H}}}}(\breve{\ell})\right)
$$

$\operatorname{over} C_{\Phi_{\mathcal{H}}, \delta_{\mathcal{H}}}$. 
(2) For any $\gamma \in \Gamma_{\widetilde{\Phi}_{\tilde{\mathcal{H}}}}$, $\Phi_{\mathcal{H}}$, we have a commutative diagram

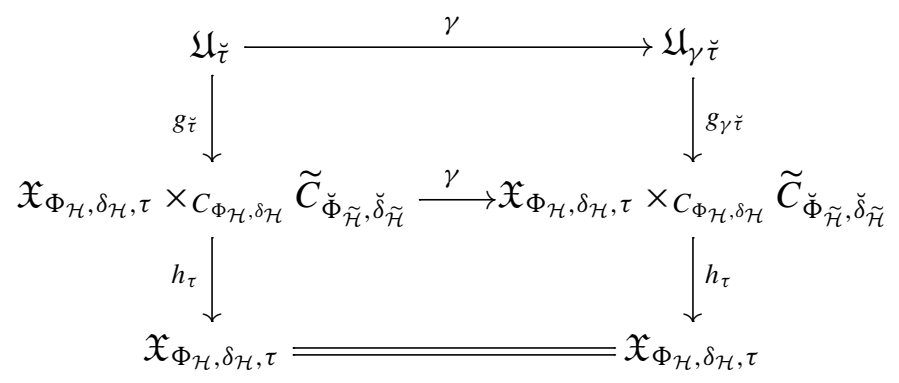

of formal schemes, (naturally) compatible with the commutative diagram

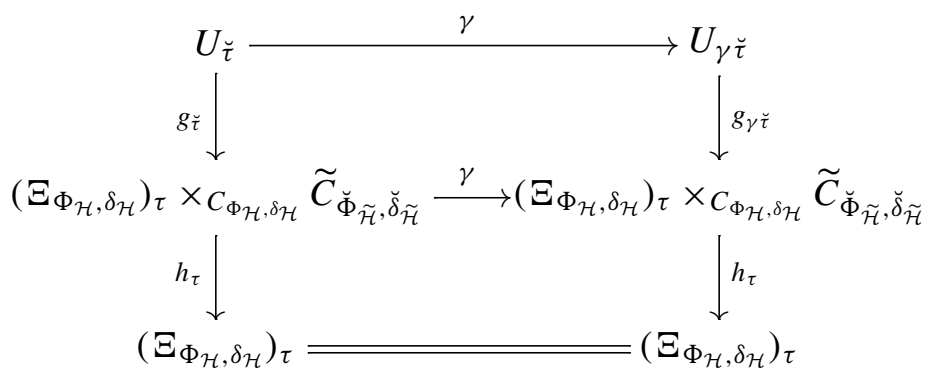

of their supports. Then (4.7) and (4.8) are compatible with the canonical isomorphisms $\gamma^{*} \mathrm{O}_{\mathfrak{U}_{y_{\tau}}} \rightarrow \mathrm{O}_{\mathfrak{U}_{\tau}}$ induced by the canonical isomorphisms $\gamma^{*} \Psi_{\breve{\Phi}_{\tilde{\mathcal{H}}}, \breve{\delta}_{\tilde{\mathcal{H}}}}(\gamma \breve{\ell}) \stackrel{\sim}{\longrightarrow} \Psi_{\breve{\Phi}_{\tilde{\mathcal{H}}}, \breve{\delta}_{\tilde{\mathcal{H}}}}(\breve{\ell})$ over $\widetilde{C}_{\breve{\Phi}_{\tilde{\mathcal{H}}}, \breve{\delta}_{\tilde{\mathcal{H}}}}$.

(3) For any integer $d \geq 0$, if $\breve{\tau}^{\prime}$ is a face of $\breve{\tau}$, then the canonical morphism $R^{d}\left(f_{\breve{\tau}}^{\text {tor }}\right)_{*} \mathrm{O}_{\mathfrak{U}_{\bar{\tau}}} \rightarrow R^{d}\left(f_{\bar{\tau}^{\prime}}^{\text {tor }}\right)_{*} \mathrm{O}_{\mathfrak{U}_{\bar{\tau}^{\prime}}}$ induced by restriction from $\mathfrak{U}_{\bar{\tau}}$ to $\mathfrak{U}_{\bar{\tau}^{\prime}}$ corresponds to the morphism

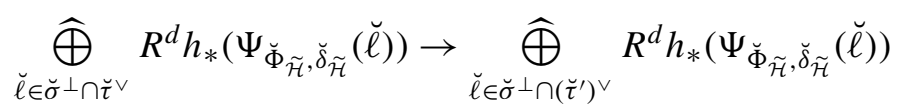

over $C_{\Phi_{\mathcal{H}}, \delta_{\mathcal{H}}}$, and the canonical morphism $R^{d}\left(f_{\breve{\tau}}^{\text {tor }}\right)_{*} \mathrm{O}_{U_{\breve{\tau}}} \rightarrow R^{d}\left(f_{\breve{\tau}^{\prime}}^{\text {tor }}\right)_{*} \mathrm{O}_{U_{\breve{\tau}^{\prime}}}$ induced by restriction from $U_{\breve{\tau}}$ to $U_{\breve{\tau}^{\prime}}$ corresponds to the morphism

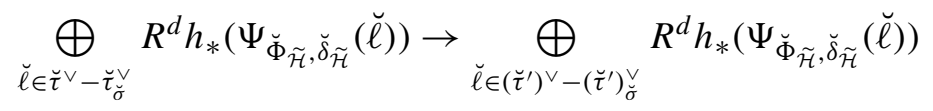

over $C_{\Phi_{\mathcal{H}}, \delta_{\mathcal{H}}}$. Both of these morphisms send $R^{d} h_{*}\left(\Psi_{\breve{\Phi}_{\widetilde{\mathcal{H}}}, \breve{\delta}_{\tilde{\mathcal{H}}}}(\breve{\ell})\right)$ (identically) to $R^{d} h_{*}\left(\Psi_{\breve{\Phi}_{\tilde{\mathcal{H}}}, \breve{\delta}_{\tilde{\mathcal{H}}}}(\breve{\ell})\right)$ when it is defined on both sides, and to zero otherwise.

4B. Relative cohomology of structural sheaves. Using (4.5), we shall identify $\left(\mathrm{M}_{\mathcal{H}}^{\text {tor }}\right)_{\mathrm{Z}_{\left[\left(\Phi_{\mathcal{H}}, \delta_{\mathcal{H}}, \tau\right)\right]}}$ with $\mathfrak{X}_{\Phi_{\mathcal{H}}, \delta_{\mathcal{H}}, \tau}$, and identify $\mathrm{Z}_{\left[\left(\Phi_{\mathcal{H}}, \delta_{\mathcal{H}}, \tau\right)\right]}$ with $\left(\Xi_{\Phi_{\mathcal{H}}, \delta_{\mathcal{H}}}\right)_{\tau}$. For simplicity of notation, we shall use $\mathfrak{X}_{\Phi_{\mathcal{H}}, \delta_{\mathcal{H}}, \tau}$ and $Z_{\left[\left(\Phi_{\mathcal{H}}, \delta_{\mathcal{H}}, \tau\right)\right]}$ more often than their counterparts. 
Recall that $C_{\Phi_{\mathcal{H}}, \delta_{\mathcal{H}}}$ is an abelian scheme over the moduli problem $\mathrm{M}_{\mathcal{H}}^{\mathrm{Z}_{\mathcal{H}}}$ (see Definition 1.17). Let $\left(A, \lambda_{A}, i_{A}, \alpha_{\mathcal{H}_{h}}\right)$ be the tautological tuple over $\mathrm{M}_{\mathcal{H}}^{Z_{\mathcal{H}}}$. Let $T$ (resp. $T^{\vee}$ ) be the split torus with character group $X$ (resp. $Y$ ). For simplicity of notation, we shall denote the pullbacks of $A, A^{\vee}, T$, and $T^{\vee}$, respectively, by the same symbols. The pullback of $G$ (resp. $G^{\vee}$ ) to $\mathfrak{X}_{\Phi_{\mathcal{H}}, \mathrm{Z}_{\mathcal{H}}, \tau}$ is an extension of $A$ (resp. $A^{\vee}$ ) by $T$ (resp. $T^{\vee}$ ), and this extension is a pullback of the tautological extension $G^{\natural}$ (resp. $G^{\vee, \natural)}$ over $C_{\Phi_{\mathcal{H}}, \delta_{\mathcal{H}}}$. For simplicity, we shall also denote the pullbacks of $G^{\natural}$ and $G^{\vee, \natural}$, respectively, by the same symbols.

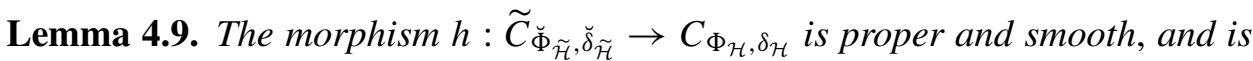
a torsor under the pullback to $C_{\Phi_{\mathcal{H}}, \delta_{\mathcal{H}}}$ of an abelian scheme $\mathbb{Z}_{(\square)}^{\times}$-isogenous to $\underline{\operatorname{Hom}}_{\mathcal{O}}(\tilde{X}, A)^{\circ} \rightarrow \mathrm{M}_{\mathcal{H}}^{\mathrm{Z}_{\mathcal{H}}}$.

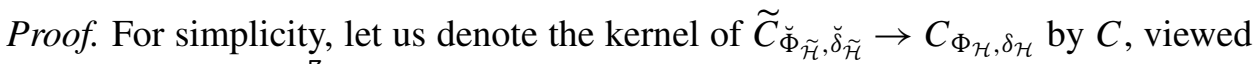
as a scheme over $\mathrm{M}_{\mathcal{H}}^{\mathrm{Z}_{\mathcal{H}}}$.

While the abelian scheme $\widetilde{C}_{\breve{\Phi}_{\widetilde{\mathcal{H}}}, \breve{\delta}_{\widetilde{\mathcal{H}}}} \rightarrow \mathrm{M}_{\mathcal{H}}^{Z_{\mathcal{H}}}$ parametrizes liftings of pairs of the form $\left(\breve{c}: \breve{X} \rightarrow A^{\vee}, \breve{c}^{\vee}: \breve{Y} \rightarrow A\right) \rightarrow \mathrm{M}_{\mathcal{H}}^{Z_{\mathcal{H}}}$ satisfying the compatibility $\breve{c} \breve{\phi}=\lambda_{A} \breve{c}^{\vee}$ and the liftability and pairing conditions, and while the abelian scheme $C_{\Phi_{\mathcal{H}}, \delta_{\mathcal{H}}} \rightarrow \mathrm{M}_{\mathcal{H}}^{Z_{\mathcal{H}}}$ parametrizes liftings of pairs of the form $\left(c: X \rightarrow A^{\vee}, c^{\vee}: Y \rightarrow A\right)$ satisfying the compatibility $c \phi=\lambda_{A} c^{\vee}$ and the liftability and pairing conditions, the scheme $C \rightarrow \mathrm{M}_{\mathcal{H}}^{Z_{\mathcal{H}}}$ parametrizes lifts of pairs of the form $\left(\tilde{c}: \tilde{X} \rightarrow A^{\vee}, \tilde{c}^{\vee}: \widetilde{Y} \rightarrow A\right)$ satisfying the compatibility $\tilde{c} \tilde{\phi}=\lambda_{A} \tilde{c}^{\vee}$ and the liftability and pairing conditions induced by the

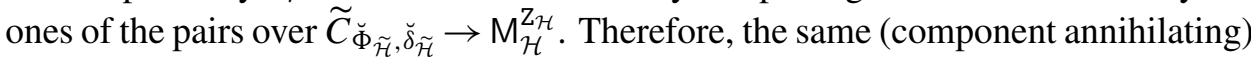
argument in [Lan 2008, §6.2.3-6.2.4] shows that the kernel $C$ of $h$ is an abelian scheme $\mathbb{Z}_{(\square)}^{\times}$-isogenous to $\underline{\operatorname{Hom}}_{\mathcal{O}}(\tilde{X}, A)^{\circ}$.

Consequently, all geometric fibers of $h$ are smooth and have the same dimension

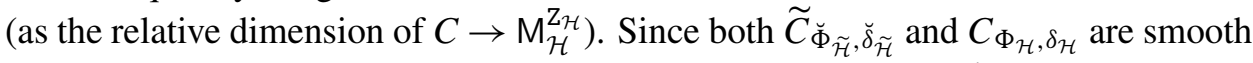
over $\mathrm{S}_{0}$, the morphism $h$ is smooth by [EGA IV $\left.\mathrm{IV}_{3} 1966,15.4 .2 \mathrm{e}^{\prime}\right) \Rightarrow \mathrm{b}$ )] and [EGA $\mathrm{IV}_{4}$ 1967, 17.5.1 b) $\Rightarrow$ a)]. By [Bosch et al. 1990, §2.2, Proposition 14], smooth morphisms between schemes have sections étale locally. This shows that $h$ is a torsor under the pullback of $C$ to $C_{\Phi_{\mathcal{H}}, \delta_{\mathcal{H}}}$. (Regardless of this argument, the

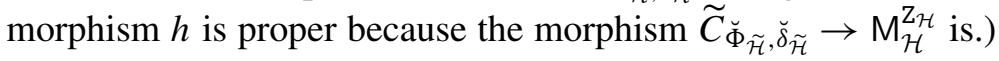

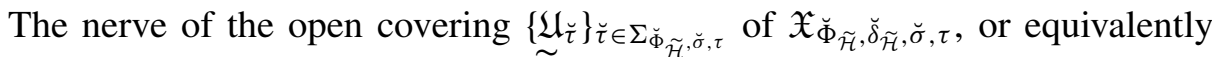

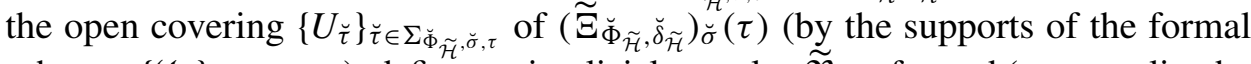

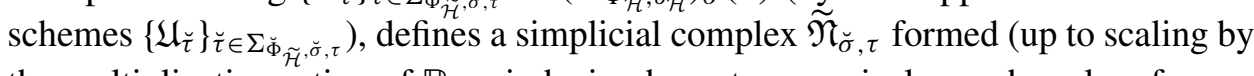
the multiplicative action of $\mathbb{R}_{>0}$, inducing homotopy equivalences harmless for our purpose) by the union of the cones $\breve{\tau}$ in $\Sigma_{\breve{\Phi}_{\widetilde{\mathcal{H}}}, \breve{\sigma}, \tau}$ (with natural incidence relations among their closures inherited from their realizations as locally closed subsets of $\left.\left(\mathbf{S}_{\Phi_{\widetilde{\mathcal{H}}}}\right)_{\mathbb{R}}^{\vee}\right)$. Then the nerve of the open covering

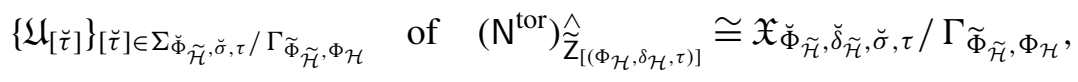


or equivalently the open covering

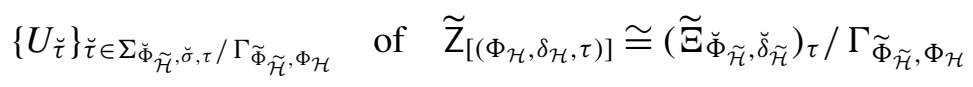

of the supports of formal schemes, is naturally identified with $\mathfrak{N}_{\breve{\sigma}, \tau}:=\widetilde{\mathfrak{N}}_{\breve{\sigma}, \tau} / \Gamma_{\widetilde{\Phi}_{\tilde{\mathcal{H}}}, \Phi_{\mathcal{H}}}$.

The simplicial complex $\widetilde{\mathfrak{N}}_{\breve{\sigma}, \tau}$ has a closed covering by the closures $\breve{\tau}^{\mathrm{cl}}$ (in $\widetilde{\mathfrak{N}}_{\breve{\sigma}, \tau}$ ) of the cones $\breve{\tau}$ in $\Sigma_{\breve{\Phi}_{\widetilde{\mathcal{H}}}, \breve{\sigma}, \tau}$, which induces a closed covering of $\mathfrak{N}_{\breve{\sigma}, \tau}$ by the closures $[\breve{\tau}]^{\mathrm{cl}}$ (in $\mathfrak{N}_{\breve{\sigma}, \tau}$ ) of the subsets $[\breve{\tau}]$ of $\Sigma_{\breve{\Phi}}, \breve{\mathcal{H}}, \tau / \Gamma_{\widetilde{\Phi}_{\tilde{\mathcal{H}}}}, \Phi_{\mathcal{H}}$. For any sheaf $\mathcal{M}$ on

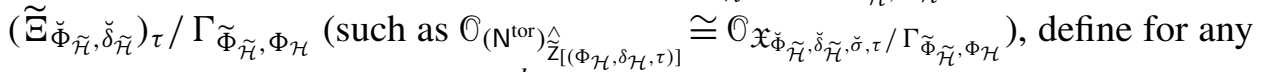
integer $d \geq 0$ the local system $\mathscr{H}^{d}(\mathcal{M})$ on $\mathfrak{N}_{\breve{\sigma}, \tau}$ which associates with each $[\breve{\tau}]$ in $\Sigma_{\breve{\Phi}_{\tilde{\mathcal{H}}}, \breve{\sigma}, \tau} / \Gamma_{\widetilde{\Phi}_{\widetilde{\mathcal{H}}}, \Phi_{\mathcal{H}}}$ the coefficients

$$
\mathscr{H}^{d}(\mathcal{M})\left([\breve{\tau}]^{\mathrm{cl}}\right):=H^{d}\left(U_{[\breve{\tau}]}, M_{U_{[\breve{\tau}]}}\right) .
$$

Then, by [Godement 1958, II, 5.4.1], there is a spectral sequence

$$
E_{2}^{c, d}:=H^{c}\left(\mathfrak{N}_{\breve{\sigma}, \tau}, \mathscr{H}^{d}(\mathcal{M})\right) \Rightarrow H^{c+d}\left(\left(\widetilde{\Xi}_{\breve{\Phi}_{\widetilde{\mathcal{H}}}, \breve{\delta}_{\widetilde{\mathcal{H}}}}\right)_{\tau} / \Gamma_{\widetilde{\Phi}_{\widetilde{\mathcal{H}}}, \Phi_{\mathcal{H}}}, \mathcal{M}\right) .
$$

The construction of $\mathfrak{N}_{\breve{\sigma}, \tau}$ depends only on the cone decomposition $\Sigma_{\breve{\Phi}_{\widetilde{\mathcal{H}}}, \breve{\sigma}, \tau}$, while the constructions of both $\mathscr{H}^{d}(\mathcal{M})$ and the spectral sequence (4.10) are compatible with restrictions to affine open subschemes of $Z_{\left[\left(\Phi_{\mathcal{H}}, \delta_{\mathcal{H}}, \tau\right)\right]}$. Therefore, we can define the sheaves $\underline{\mathcal{H}}^{d}(\mathcal{M})$ (of local systems on $\mathfrak{N}_{\breve{\sigma}, \tau}$ ) over $\mathrm{Z}_{\left[\left(\Phi_{\mathcal{H}}, \delta_{\mathcal{H}}, \tau\right)\right]}$, and obtain a spectral sequence

$$
E_{2}^{c, d}:=H^{c}\left(\mathfrak{N}_{\breve{\sigma}, \tau}, \underline{\mathscr{H}}^{d}(\mathcal{M})\right) \Rightarrow R^{c+d} f_{*}^{\text {tor }}(\mathcal{M}) .
$$

Here $H^{c}\left(\mathfrak{N}_{\breve{\sigma}, \tau}, \underline{\mathcal{H}}^{d}(\mathcal{M})\right)$ is interpreted as a sheaf on $\mathrm{Z}_{\left[\left(\Phi_{\mathcal{H}}, \delta_{\mathcal{H}}, \tau\right)\right]}$, and the formation of (4.11) is compatible with morphisms in $M$. In particular, we have compatible spectral sequences

$$
E_{2}^{c, d}:=H^{c}\left(\mathfrak{N}_{\breve{\sigma}, \tau}, \underline{\mathscr{H}}^{d}\left(\widehat{O}_{\left(\mathrm{N}^{\text {tor }}\right)} \hat{\tilde{\mathrm{z}}}_{\left[\left(\Phi_{\mathcal{H}}, \delta_{\mathcal{H}}, \tau\right)\right]}\right)\right) \Rightarrow R^{c+d} f_{*}^{\text {tor }}\left(\mathcal{O}_{\left(\mathrm{N}^{\text {tor }}\right)} \hat{\tilde{\mathrm{Z}}}_{\left[\left(\Phi_{\mathcal{H}}, \delta_{\mathcal{H}}, \tau\right)\right]}\right)
$$

and

$$
E_{2}^{c, d}:=H^{c}\left(\mathfrak{N}_{\breve{\sigma}, \tau}, \underline{\mathscr{H}}^{d}\left(\mathcal{O}_{\left.\left.\widetilde{Z}_{\left[\left(\Phi_{\mathcal{H}}, \delta \mathcal{H}\right.\right.}, \tau\right)\right]}\right)\right) \Rightarrow R^{c+d} f_{*}^{\text {tor }}\left(\complement_{\widetilde{Z}_{\left[\left(\Phi_{\mathcal{H}}, \delta_{\mathcal{H}}, \tau\right)\right]}}\right) .
$$

To calculate the left-hand sides of (4.12) and (4.13), we define the sheaves

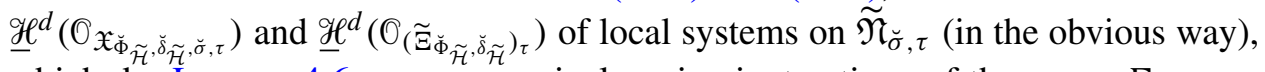
which, by Lemma 4.6, carry canonical equivariant actions of the group $\Gamma_{\widetilde{\Phi}_{\tilde{\mathcal{H}}}}, \Phi_{\mathcal{H}}$,

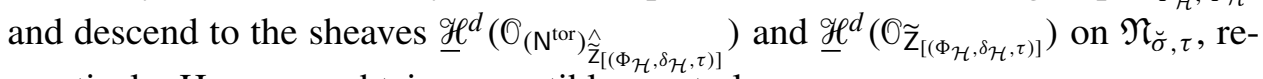
spectively. Hence we obtain compatible spectral sequences

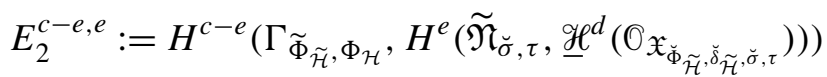

$$
\begin{aligned}
& \Rightarrow H^{c}\left(\mathfrak{N}_{\breve{\sigma}, \tau}, \underline{\mathscr{H}}^{d}\left(\mathrm{O}_{\left(\mathrm{N}^{\text {tor }}\right)} \hat{\tilde{\mathrm{z}}}_{\left[\left(\Phi_{\mathcal{H}}, \delta \mathcal{H}, \tau\right)\right]}\right)\right)
\end{aligned}
$$


and

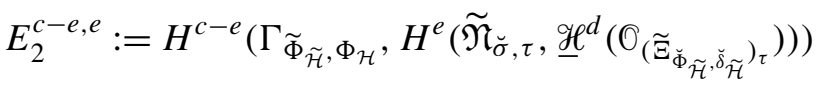

$$
\begin{aligned}
& \Rightarrow H^{c}\left(\mathfrak{N}_{\breve{\sigma}, \tau}, \underline{\mathscr{H}}^{d}\left(\mathrm{O}_{\widetilde{Z}_{\left[\left(\Phi_{\mathcal{H}}, \delta \mathcal{H}, \tau\right]\right.}}\right)\right) .
\end{aligned}
$$

Lemma 4.16. For any $d \geq 0$, the canonical morphisms

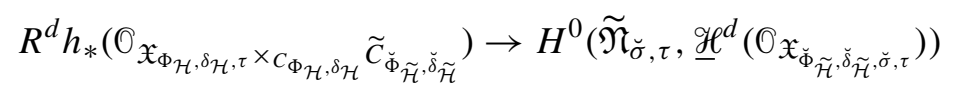

and

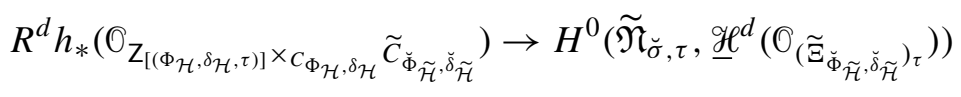

are isomorphisms compatible with each other. Moreover, for any integer e $>0$, we have

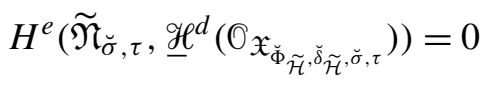

and

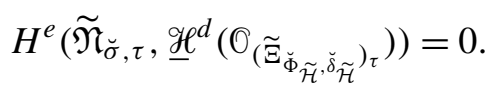

Proof. By (4.7), we have

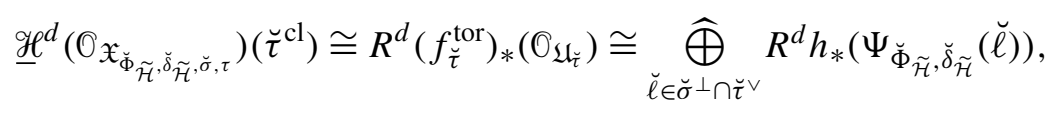

and for any face $\breve{\tau}^{\prime}$ of $\breve{\tau}$, the canonical morphism

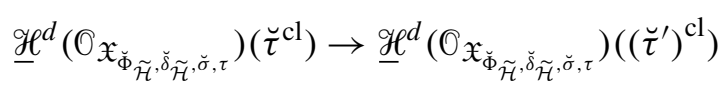

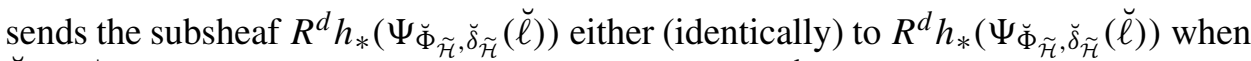
$\breve{\ell} \in \breve{\sigma}^{\perp} \cap\left(\breve{\tau}^{\prime}\right)^{\vee}$, or to zero otherwise. Since $\bigcup_{\breve{\ell} \in \breve{\tau}^{\vee}} \breve{\tau}^{\mathrm{cl}}=\bigcup_{\breve{\ell} \in \breve{\tau}^{\vee}} \breve{\tau}$ is a convex subset of $\tilde{\mathfrak{N}}_{\breve{\sigma}, \tau}$ for any given $\breve{\ell} \in \breve{\sigma}^{\perp}$, this shows (4.19) as usual (by the argument in [Kempf

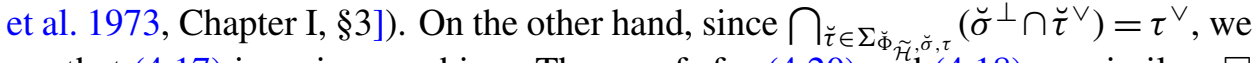
see that (4.17) is an isomorphism. The proofs for (4.20) and (4.18) are similar.

Lemma 4.21. The topological space $\mathfrak{N}_{\breve{\sigma}, \tau}$ is homotopic to the real torus

$$
\mathbf{T}_{\widetilde{\Phi}_{\widetilde{\mathcal{H}}}, \Phi_{\mathcal{H}}}:=\left(\Gamma_{\widetilde{\Phi}_{\widetilde{\mathcal{H}}}, \Phi_{\mathcal{H}}}\right)_{\mathbb{R}}^{\vee} / \Gamma_{\widetilde{\Phi}_{\widetilde{\mathcal{H}}}, \Phi_{\mathcal{H}}},
$$

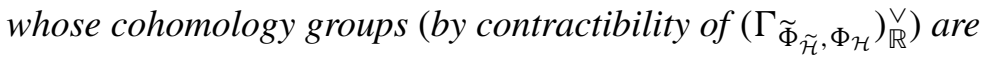

$$
H^{j}\left(\mathbf{T}_{\widetilde{\Phi}_{\tilde{\mathcal{H}}}, \Phi_{\mathcal{H}}}, \mathbb{Z}\right) \cong H^{j}\left(\Gamma_{\widetilde{\Phi}_{\tilde{\mathcal{H}}}, \Phi_{\mathcal{H}}}, \mathbb{Z}\right) \cong \bigwedge^{j}\left(\operatorname{Hom}_{\mathbb{Z}}\left(\Gamma_{\widetilde{\Phi}_{\tilde{\mathcal{H}}}, \Phi_{\mathcal{H}}}, \mathbb{Z}\right)\right)
$$

for any $j \geq 0$. Over $C_{\Phi_{\mathcal{H}}, \delta_{\mathcal{H}}}$, we have a canonical isomorphism

$$
H^{j}\left(\Gamma_{\widetilde{\Phi}_{\tilde{\mathcal{H}}}, \Phi_{\mathcal{H}}}, \mathbb{Z}\right) \otimes_{\mathbb{Z}} \mathcal{O}_{C_{\Phi_{\mathcal{H}}, \delta_{\mathcal{H}}}} \cong \bigwedge^{j}\left(\operatorname{Hom}_{\mathcal{O}}\left(Q^{\vee}, \underline{\operatorname{Lie}}_{T^{\vee} / C_{\Phi_{\mathcal{H}}, \delta_{\mathcal{H}}}}\right)\right) .
$$


Proof. Since $\widetilde{\sigma}$ is a top-dimensional cone in $\mathbf{P}_{\widetilde{\Phi}_{\tilde{\mathcal{H}}}}^{+}$, any $\breve{\tau} \in \Sigma_{\breve{\Phi}} \breve{\mathcal{H}}_{\tilde{\sigma}}, \breve{\sigma}, \tau$ (which has $\breve{\sigma}$ as a face) is generated by $\breve{\sigma}$ and some rational basis vectors not contained in the image of the first morphism in (3.7). Moreover, the image of $\breve{\tau}$ under the second morphism in (3.7) is contained in $\tau \subset \mathbf{P}_{\Phi_{\mathcal{H}}}^{+}$. By choosing some (noncanonical) splitting of $s_{\breve{X}} \otimes_{\mathbb{Z}} \mathbb{Q}: \breve{X} \otimes_{\mathbb{Z}} \mathbb{Q} \rightarrow \widetilde{X} \otimes_{\mathbb{Z}} \mathbb{Q}$, we can decompose the real vector space $\left(\mathbf{S}_{\Phi_{\tilde{\mathcal{H}}}}\right)_{\mathbb{R}}^{\vee}$ (noncanonically) as a direct sum $\left(\mathbf{S}_{\widetilde{\Phi}_{\tilde{\mathcal{H}}}}\right)_{\mathbb{R}}^{\vee} \oplus\left(\Gamma_{\widetilde{\Phi}_{\tilde{\mathcal{H}}}, \Phi_{\mathcal{H}}}\right)_{\mathbb{R}}^{\vee} \oplus\left(\mathbf{S}_{\Phi_{\mathcal{H}}}\right)_{\mathbb{R}}^{\vee}$, on which the action of $\Gamma_{\widetilde{\Phi}_{\tilde{\mathcal{H}}}}, \Phi_{\mathcal{H}}$ is realized by its canonical translation action on the second factor. Along the directions of $\left(\mathbf{S}_{\widetilde{\Phi}_{\tilde{\mathcal{H}}}}\right)_{\mathbb{R}}^{\vee}$ and $\left(\mathbf{S}_{\Phi_{\mathcal{H}}}\right)_{\mathbb{R}}^{\vee}$, we can contract the simplicial complex $\tilde{\mathfrak{N}}_{\breve{\sigma}, \tau}$ (say, towards some arbitrarily chosen points in the convex sets $\widetilde{\sigma}$ and $\tau$ ) in a way compatible with the actions of $\Gamma \widetilde{\Phi}_{\widetilde{\mathcal{H}}}, \Phi_{\mathcal{H}}$. Therefore, $\mathfrak{N}_{\breve{\sigma}, \tau}=\widetilde{\mathfrak{N}}_{\breve{\sigma}, \tau} / \Gamma_{\widetilde{\Phi}_{\tilde{\mathcal{H}}}}, \Phi_{\mathcal{H}}$ is homotopic to the real torus $\mathbf{T}_{\widetilde{\Phi}_{\tilde{\mathcal{H}}}, \Phi_{\mathcal{H}}}=\left(\Gamma \widetilde{\Phi}_{\tilde{\mathcal{H}}}, \Phi_{\mathcal{H}}\right)_{\mathbb{R}}^{\vee} / \Gamma_{\widetilde{\Phi}_{\tilde{\mathcal{H}}}}, \Phi_{\mathcal{H}}$.

The canonical isomorphism (4.22) then follows from the composition of the following canonical isomorphisms:

$$
\begin{aligned}
& H^{j}\left(\Gamma_{\widetilde{\Phi}_{\tilde{\mathcal{H}}}, \Phi_{\mathcal{H}}}, \mathbb{Z}\right) \otimes_{\mathbb{Z}} 0_{C_{\Phi_{\mathcal{H}}, \delta_{\mathcal{H}}}} \cong\left(\bigwedge^{j}\left(\operatorname{Hom}_{\mathbb{Z}}\left(\Gamma_{\widetilde{\Phi}_{\tilde{\mathcal{H}}}, \Phi_{\mathcal{H}}}, \mathbb{Z}\right)\right)\right) \otimes_{\mathbb{Z}} 0_{C_{\Phi_{\mathcal{H}}, \delta_{\mathcal{H}}}} \\
& \cong\left(\bigwedge^{j}\left(\operatorname{Hom}_{\mathbb{Z}}\left(\operatorname{Hom}_{\mathcal{O}}(\tilde{X}, X), \mathbb{Z}_{(\square)}\right)\right)\right) \otimes_{\mathbb{Z}_{(\square)}} O_{C_{\Phi_{\mathcal{H}}, \delta_{\mathcal{H}}}} \\
& \cong \bigwedge^{j}\left(\operatorname{Hom}_{\mathcal{O}}\left(Q^{\vee}, \operatorname{Hom}_{\mathbb{Z}}\left(Y, \mathscr{O}_{C_{\Phi_{\mathcal{H}}, \delta_{\mathcal{H}}}}\right)\right)\right) \\
& \left.\cong \bigwedge^{j}\left(\operatorname{Hom}_{\mathcal{O}}\left(Q^{\vee}, \underline{\operatorname{Lie}}_{T^{\vee}} / C_{\Phi_{\mathcal{H}}, \delta_{\mathcal{H}}}\right)\right)\right) \text {. }
\end{aligned}
$$

Lemma 4.23. There are compatible canonical isomorphisms

and

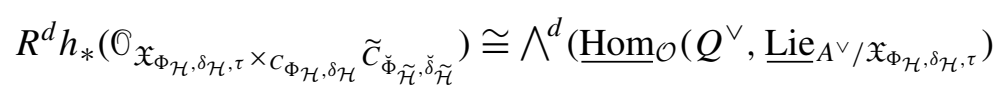

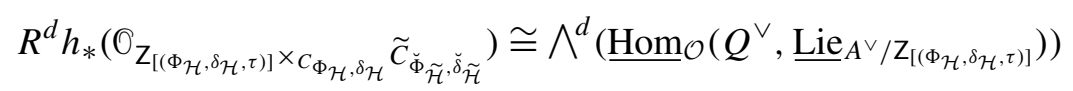

for any integer $d \geq 0$.

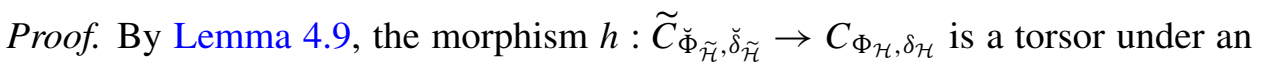
abelian scheme $\mathbb{Z}_{(\square)}^{\times}$-isogenous to $\underline{\operatorname{Hom}}_{\mathcal{O}}(Q, A)^{\circ}$ (and hence has a section étale locally). Since the cohomology of abelian schemes (with coefficients in the structural sheaves) are free and are compatible with arbitrary base changes (see [Berthelot et al. 1982, Proposition 2.5.2; Mumford 1970, §5]), we obtain compatible canonical isomorphisms

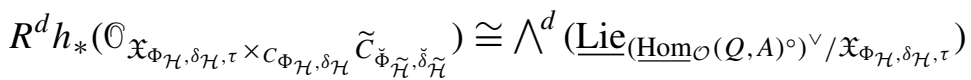

$$
\begin{aligned}
& \cong \bigwedge^{d}\left(\underline{\operatorname{Hom}}_{\mathcal{O}}\left(Q^{\vee}, \underline{\operatorname{Lie}}_{A^{\vee} / \mathfrak{X}_{\Phi_{\mathcal{H}}, \delta_{\mathcal{H}}, \tau}}\right)\right),
\end{aligned}
$$

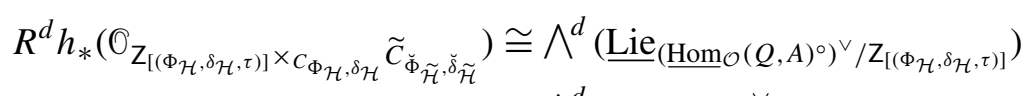

$$
\begin{aligned}
& \cong \bigwedge^{d}\left(\underline{\operatorname{Hom}}_{\mathcal{O}}\left(Q^{\vee}, \underline{\operatorname{Lie}}_{A^{\vee}} / \mathrm{Z}_{\left[\left(\Phi_{\mathcal{H}}, \delta \mathcal{H}, \tau\right)\right]}\right)\right)
\end{aligned}
$$

for any integer $d \geq 0$. 
Proposition 4.24. There are compatible canonical isomorphisms

$$
\begin{aligned}
& H^{c}\left(\mathfrak{N}_{\breve{\sigma}, \tau}, \underline{\mathscr{H}}^{d}\left(\mathcal{O}_{\left(\mathrm{N}^{\text {tor }}\right)} \hat{\tilde{\mathrm{z}}}_{\left[\left(\Phi_{\mathcal{H}}, \delta_{\mathcal{H}}, \tau\right)\right]}\right)\right) \cong\left(\bigwedge^{c}\left(\underline{\operatorname{Hom}}_{\mathcal{O}}\left(Q^{\vee}, \underline{\operatorname{Lie}}_{T^{\vee} / \mathfrak{X}_{\Phi_{\mathcal{H}}, \delta_{\mathcal{H}}, \tau}}\right)\right)\right) \otimes_{\mathcal{O}_{\mathfrak{X}^{\prime}} \Phi_{\mathcal{H}}, \delta_{\mathcal{H}}, \tau} \\
& \left(\bigwedge ^ { d } \left(\underline { \operatorname { H o m } } _ { \mathcal { O } } \left(Q^{\vee}, \underline{\operatorname{Lie}}_{\left.\left.\left.A^{\vee} / \mathfrak{X}_{\Phi_{\mathcal{H}}, \delta_{\mathcal{H}}, \tau}\right)\right)\right)}(\right.\right.\right.
\end{aligned}
$$

and

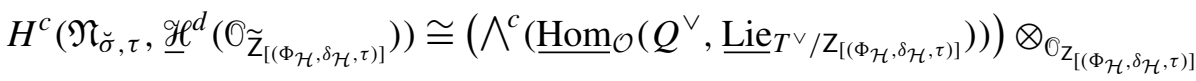

$$
\begin{aligned}
& \left(\bigwedge^{d}\left(\underline{\operatorname{Hom}}_{\mathcal{O}}\left(Q^{\vee}, \underline{\operatorname{Lie}}_{\left.\left.A^{\vee} / \mathrm{Z}_{\left[\left(\Phi_{\mathcal{H}}, \delta \mathcal{H}\right.\right.}, \tau\right)\right]}\right)\right)\right)
\end{aligned}
$$

for any integers $c, d \geq 0$.

Proof. By Lemma 4.16, the spectral sequences (4.14) and (4.15) degenerate and show that for any integers $c$ and $d$ we have compatible canonical isomorphisms

$$
\begin{aligned}
& H^{c}\left(\mathfrak{N}_{\breve{\sigma}, \tau}, \underline{\mathscr{H}}^{d}\left(\mathcal{O}_{\left(\mathrm{N}^{\text {tor }}\right)} \hat{\tilde{\mathrm{z}}}_{\left[\left(\Phi_{\mathcal{H}}, \delta_{\mathcal{H}}, \tau\right)\right]}\right)\right)
\end{aligned}
$$

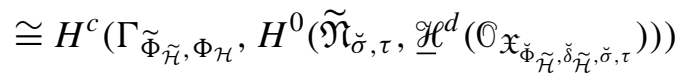

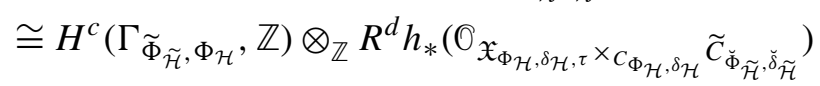

and

$$
\begin{aligned}
& H^{c}\left(\mathfrak{N}_{\breve{\sigma}, \tau}, \underline{\mathscr{H}}^{d}\left(\widetilde{O}_{\left.\left.\widetilde{Z}_{\left[\left(\Phi_{\mathcal{H}}, \delta_{\mathcal{H}}, \tau\right)\right]}\right)\right)}\right.\right.
\end{aligned}
$$

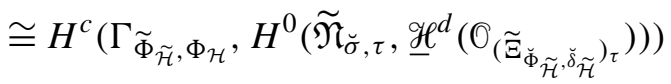

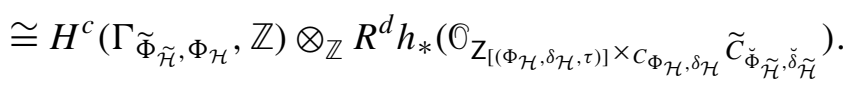

Now combine (4.27) and (4.28) with Lemmas 4.21 and 4.23.

Lemma 4.29. The spectral sequence (4.12) degenerates at $E_{2}$ terms. Consequently, since the choice of the stratum $\mathrm{Z}_{\left[\left(\Phi_{\mathcal{H}}, \delta_{\mathcal{H}}, \tau\right)\right]}$ is arbitrary, by Grothendieck's fundamental theorem [EGA III 1 1961, 4.1.5] (and by fpqc descent for the property of local freeness [SGA 1 1971, VIII, 1.11]), the sheaf $R^{b} f_{*}^{\text {tor }}\left(O_{\mathrm{N}^{\text {tor }}}\right)$ is locally free of the same rank as $\wedge^{b}\left(\underline{\operatorname{Hom}}_{\mathcal{O}}\left(Q^{\vee}, \underline{\operatorname{Lie}}_{G^{\vee}} / \mathrm{M}_{\mathcal{H}}^{\text {tor }}\right)\right)$ over $\mathrm{M}_{\mathcal{H}}^{\text {tor }}$.

If, for every maximal point $s$ of $\mathrm{Z}_{\left[\left(\Phi_{\mathcal{H}}, \delta_{\mathcal{H}}, \tau\right)\right]}$ (see [Grothendieck 1971, 0 2.1.2]), we have

$$
\begin{aligned}
& \operatorname{dim}_{k(s)}\left(\left(R^{b} f_{*}^{\text {tor }}\left(\widetilde{\mathrm{Z}}_{\left[\widetilde{\mathrm{Z}}_{\left.\left[\Phi_{\mathcal{H}}, \delta \mathcal{H}, \tau\right)\right]}\right.}\right)\right) \otimes_{\left.\mathcal{O}_{[}\left(\Phi_{\mathcal{H}}, \delta_{\mathcal{H}}, \tau\right)\right]} k(s)\right)
\end{aligned}
$$

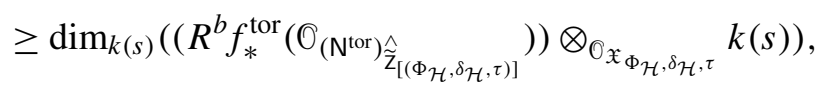


then the spectral sequence (4.13) degenerates at $E_{2}$ terms as well, and there is a canonical isomorphism

$$
R^{b} f_{*}^{\text {tor }}\left(\mathcal{O}_{\mathrm{N}^{\text {tor }}}\right) \otimes_{\mathcal{O}_{\mathrm{M}_{\mathcal{H}}}} \mathcal{O}_{\mathrm{Z}_{\left[\left(\Phi_{\mathcal{H}}, \delta_{\mathcal{H}}, \tau\right)\right]}} \stackrel{\sim}{\rightarrow} R^{b} f_{*}^{\text {tor }}\left(\widetilde{O}_{\left.\left.\widetilde{\mathrm{Z}}_{\left[\left(\Phi_{\mathcal{H}}, \delta \mathcal{H}\right.\right.}, \tau\right)\right]}\right) .
$$

Proof. Let $\operatorname{Spf}(R, I)$ be any connected affine open formal subscheme of $\mathfrak{X}_{\Phi_{\mathcal{H}}, \delta_{\mathcal{H}}, \tau}$, with the ideal of definition $I$ satisfying $\operatorname{rad}(I)=I$ for simplicity. Since $\mathrm{M}_{\mathcal{H}}^{\text {tor }}$ is smooth and of finite type over $\mathrm{S}_{0}=\operatorname{Spec}\left(\mathcal{O}_{F_{0},(\square)}\right)$, the ring $R$ is a noetherian domain. (See [Matsumura 1980, 33.I and 34.A].) Since $Z_{\left[\left(\Phi_{\mathcal{H}}, \delta_{\mathcal{H}}, \tau\right)\right]}$ is a smooth subscheme of $\mathrm{M}_{\mathcal{H}}^{\text {tor }}$, the quotient $R / I$ is also a noetherian domain. Let $K:=\operatorname{Frac}(R)$ and $k:=\operatorname{Frac}(R / I)$ be the fraction fields. By abuse of notation, we shall denote pullbacks of schemes to $\operatorname{Spec}(K)$ (resp. $\operatorname{Spec}(k)$ ) by the subscript $K$ (resp. $k$ ).

Since we have an exact sequence

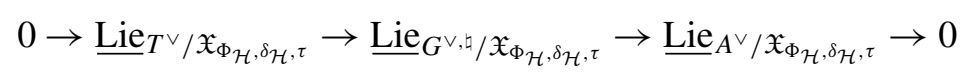

of locally free sheaves, we have an equality

$$
\begin{aligned}
\sum_{c+d=b} \operatorname{dim}_{K}\left(\bigwedge^{c}\left(\operatorname{Hom}_{\mathcal{O}}\left(Q^{\vee}, \operatorname{Lie}_{A_{K}^{\vee}}\right)\right)\right) & \otimes_{K}\left(\bigwedge^{d}\left(\operatorname{Hom}_{\mathcal{O}}\left(Q^{\vee}, \operatorname{Lie}_{T_{K}^{\vee}}\right)\right)\right) \\
& =\operatorname{dim}_{K}\left(\bigwedge^{b}\left(\operatorname{Hom}_{\mathcal{O}}\left(Q^{\vee}, \operatorname{Lie}_{G_{K}^{\vee, \natural}}\right)\right)\right) \\
& =\operatorname{dim}_{K}\left(\bigwedge^{b}\left(\operatorname{Hom}_{\mathcal{O}}\left(Q^{\vee}, \operatorname{Lie}_{G_{K}^{\vee}}\right)\right)\right),
\end{aligned}
$$

and an analogous equality with $K$ replaced with $k$.

By construction of the spectral sequences (4.12) and (4.13), by the canonical isomorphisms (4.25) and (4.26), and by the equality (4.32), we have

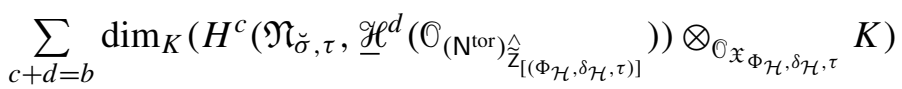

$$
\begin{aligned}
& =\operatorname{dim}_{K}\left(\bigwedge^{b}\left(\operatorname{Hom}_{\mathcal{O}}\left(Q^{\vee}, \operatorname{Lie}_{G_{K}^{\vee}}\right)\right)\right)
\end{aligned}
$$

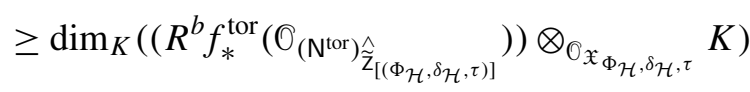

and

$$
\begin{aligned}
& \sum_{c+d=b} \operatorname{dim}_{k}\left(H^{c}\left(\mathfrak{N}_{\breve{\sigma}, \tau}, \underline{\mathscr{H}}^{d}\left(\widetilde{O}_{\widetilde{Z}_{\left[\left(\Phi_{\mathcal{H}}, \delta_{\mathcal{H}}, \tau\right)\right]}}\right)\right) \otimes_{\left.\mathcal{O}_{[}\left(\Phi_{\mathcal{H}}, \delta_{\mathcal{H}}, \tau\right)\right]} k\right) \\
& =\operatorname{dim}_{k}\left(\bigwedge^{b}\left(\operatorname{Hom}_{\mathcal{O}}\left(Q^{\vee}, \operatorname{Lie}_{G_{k}^{\vee}}\right)\right)\right) \\
& \geq \operatorname{dim}_{k}\left(R^{b} f_{*}^{\text {tor }}\left(\mathscr{O}_{\tilde{Z}_{\left[\left(\Phi_{\mathcal{H}}, \delta_{\mathcal{H}}, \tau\right)\right]}}\right) \otimes_{\mathcal{O}_{\left[\left(\Phi_{\mathcal{H}}, \delta_{\mathcal{H}}, \tau\right)\right]}} k\right) .
\end{aligned}
$$

Since the pullback of $f^{\text {tor }}$ to the open dense subscheme $M_{\mathcal{H}}$ of $M_{\mathcal{H}}^{\text {tor }}$ is simply the abelian scheme $f: \mathrm{N}^{\prime} \rightarrow \mathrm{M}_{\mathcal{H}}$, we have 


$$
\begin{aligned}
\left(R^{b} f_{*}^{\text {tor }}\left(\mathcal{O}_{\mathrm{N}^{\text {tor }}}\right)\right) \otimes_{\mathcal{O}_{\mathrm{M}_{\mathcal{H}}^{\text {tor }}}} \mathrm{O}_{\mathrm{M}_{\mathcal{H}}} & \cong R^{b} f_{*}\left(\mathrm{O}_{\mathrm{N}}\right) \\
& \cong \Lambda^{b} \underline{\mathrm{Lie}}_{\mathrm{N}^{\vee} / \mathrm{M}_{\mathcal{H}}} \cong \Lambda^{b}\left(\underline{\operatorname{Hom}}_{\mathcal{O}}\left(Q^{\vee}, \underline{\operatorname{Lie}}_{G_{\mathrm{M}_{\mathcal{H}}} / \mathrm{M}_{\mathcal{H}}}\right)\right) .
\end{aligned}
$$

Since the canonical morphism $\operatorname{Spec}(K) \rightarrow \mathrm{M}_{\mathcal{H}}^{\text {tor }}$ factors through some maximal point of $\mathrm{M}_{\mathcal{H}}$, this implies that the inequality in (4.33) is an equality, and hence that the spectral sequence (4.12) degenerates at $E_{2}$ terms after pullback to $K$. Since all $E_{2}$ terms of this spectral sequence are locally free sheaves, this shows that (4.12) degenerates at $E_{2}$ terms after pullback to $R$. Since the choice of $R$ is arbitrary, this shows that (4.12) degenerates over the whole $\mathfrak{X}_{\Phi_{\mathcal{H}}, \delta_{\mathcal{H}}, \tau}$, and hence $R^{b} f_{*}^{\text {tor }}\left(\mathrm{O}_{\mathrm{N}^{\text {tor }}}\right)$ is locally free of the same rank as $\bigwedge^{b}\left(\underline{\operatorname{Hom}}_{\mathcal{O}}\left(Q^{\vee}, \underline{\mathrm{Lie}}_{G^{\vee}} / \mathrm{M}_{\mathcal{H}}^{\text {tor }}\right)\right)$ over $\mathrm{M}_{\mathcal{H}}^{\text {tor }}$. (Nevertheless, since $f^{\text {tor }}$ is not necessarily flat, this does not imply that the formation of $R^{b} f_{*}^{\text {tor }}\left(O_{N^{\text {tor }}}\right)$ is compatible with arbitrary base change.)

Since the canonical morphism $\operatorname{Spec}(k) \rightarrow Z_{\left[\left(\Phi_{\mathcal{H}}, \delta_{\mathcal{H}}, \tau\right)\right]}$ factors through some maximal point of $Z_{\left[\left(\Phi_{\mathcal{H}}, \delta_{\mathcal{H}}, \tau\right)\right]}$, the inequality (4.30) implies that

$$
\begin{aligned}
& \operatorname{dim}_{k}\left(R^{b} f_{*}^{\text {tor }}\left(\widetilde{O}_{\widetilde{\mathbf{Z}}_{\left[\left(\Phi_{\mathcal{H}}, \delta_{\mathcal{H}}, \tau\right)\right]}}\right) \otimes_{\mathcal{O}_{\left[\left(\Phi_{\mathcal{H}}, \delta_{\mathcal{H}}, \tau\right)\right]}} k\right)
\end{aligned}
$$

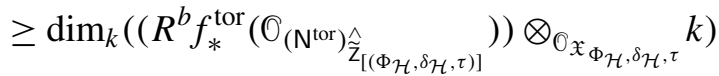

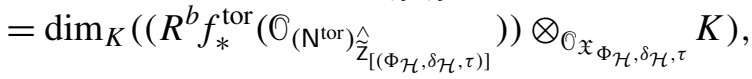

and hence the equality in (4.33) implies the equality in (4.34), because

$$
\operatorname{dim}_{k}\left(\bigwedge^{b}\left(\operatorname{Hom}_{\mathcal{O}}\left(Q^{\vee}, \operatorname{Lie}_{G_{k}^{\vee}}\right)\right)\right)=\operatorname{dim}_{K}\left(\bigwedge^{b}\left(\operatorname{Hom}_{\mathcal{O}}\left(Q^{\vee}, \operatorname{Lie}_{G_{K}^{\vee}}\right)\right)\right) .
$$

Therefore, by the same reasoning as in the case of (4.12) above, the spectral sequence (4.13) also degenerates at $E_{2}$ terms. Since the spectral sequences (4.12) and (4.13) are compatible with each other (by their very construction), their degeneracy implies that the canonical morphism

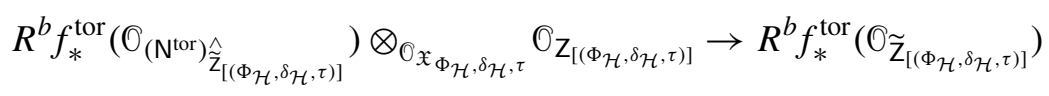

is an isomorphism (by comparing graded pieces) and induces (4.31).

Remark 4.35. By upper semicontinuity for proper flat morphisms (see [Mumford $1970, \S 5$ Corollary (a)]), the assumption (4.30) is satisfied when $f^{\text {tor }}$ is flat, or equivalently when Condition 3.17 is satisfied (by Proposition 3.18), which can be achieved by refining both $\widetilde{\Sigma}$ and $\Sigma$ (by Proposition 3.19).

Corollary 4.36. For any integer $b \geq 0$, the canonical (cup product) morphism $\Lambda^{b}\left(R^{1} f_{*}^{\text {tor }}\left(\mathrm{O}_{\mathrm{N}^{\text {tor }}}\right)\right) \rightarrow R^{b} f_{*}^{\text {tor }}\left(\mathcal{O}_{\mathrm{N}^{\text {tor }}}\right)$ is an isomorphism.

Proof. As in Lemma 4.29, by properness of $f^{\text {tor }}$, this is true if and only if it is true over the formal completion along each stratum $Z_{\left[\left(\Phi_{\mathcal{H}}, \delta_{\mathcal{H}}, \tau\right)\right]}$, which is the case because the canonical morphism induces isomorphisms on all graded pieces defined 
by spectral sequences such as (4.12), which are compatible with cup products by the very construction (see [Godement 1958, II, §5-6]).

4C. Degeneracy of the (relative) Hodge spectral sequence. As in (3c) of Theorem 2.15 , let $\underline{H}_{\log \text {-dR }}^{i}\left(\mathrm{~N}^{\text {tor }} / \mathrm{M}_{\mathcal{H}}^{\text {tor }}\right):=R^{i} f_{*}^{\text {tor }} \bar{\Omega}_{\mathrm{N}^{\text {tor }}} / \mathrm{M}_{\mathcal{H}}^{\text {tor }}$ be the (relative) $\log$ de Rham cohomology. By the definition of $\underline{H}_{\log -\mathrm{dR}}^{i}\left(\mathrm{~N}^{\text {tor }} / \mathrm{M}_{\mathcal{H}}^{\text {tor }}\right)$ as the "relative hypercohomology", the natural (Hodge) filtration on the complex $\bar{\Omega}_{\dot{N}^{\text {tor }} / \mathrm{M}_{\mathcal{H}}^{\text {tor }}}$ defines the (relative) Hodge spectral sequence (2.20):

$$
E_{1}^{a, b}:=R^{b} f_{*}^{\text {tor }}\left(\bar{\Omega}_{\mathrm{N}^{\text {tor }} / \mathrm{M}_{\mathcal{H}}^{\text {tor }}}^{a}\right) \Rightarrow \underline{H}_{\log -\mathrm{dR}}^{a+b}\left(\mathrm{~N}^{\text {tor }} / \mathrm{M}_{\mathcal{H}}^{\text {tor }}\right) .
$$

By (3a) of Theorem 2.15 (which we have proved in Section 3C), there is a canonical isomorphism

$$
\begin{aligned}
\bar{\Omega}_{\mathrm{N}^{\text {tor }} / \mathrm{M}_{\mathcal{H}}^{\text {tor }}} & \cong \bigwedge^{a}\left[\left(f^{\text {tor }}\right)^{*}\left(\underline{\operatorname{Hom}}_{\mathcal{O}}\left(Q^{\vee}, \underline{\operatorname{Lie}}_{G / \mathrm{M}_{\mathcal{H}}^{\text {tor }}}^{\vee}\right)\right]\right. \\
& \cong\left(f^{\text {tor }}\right)^{*}\left[\bigwedge^{a}\left(\underline{\operatorname{Hom}}_{\mathcal{O}}\left(Q^{\vee}, \underline{\operatorname{Lie}}_{G / \mathrm{M}_{\mathcal{H}}^{\text {tor }}}\right)\right)\right]
\end{aligned}
$$

of locally free sheaves over $\mathrm{N}^{\text {tor }}$. Then (by the projection formula [EGA I 1960, chapitre $0,5.4 .10 .1]$ ) we have canonical isomorphisms

$$
R^{b} f_{*}^{\text {tor }}\left(\overline { \Omega } _ { \mathrm { N } ^ { \text { tor } } / \mathrm { M } _ { \mathcal { H } } ^ { \text { tor } } } ^ { a } \cong ( R ^ { b } f _ { * } ^ { \text { tor } } ( \mathcal { O } _ { \mathrm { N } ^ { \text { tor } } } ) ) \otimes _ { \mathcal { O } _ { \mathrm { M } _ { \mathcal { H } } ^ { \text { tor } } } } \left(\bigwedge^{a}\left(\underline{\operatorname{Hom}}_{\mathcal{O}}\left(Q^{\vee}, \underline{\operatorname{Lie}}_{G / \mathrm{M}_{\mathcal{H}}^{\text {tor }}}^{\vee}\right)\right) .\right.\right.
$$

Lemma 4.38. If $R^{b} f_{*}^{\text {tor }}\left(\mathrm{O}_{\mathrm{N}}\right.$ tor $)$ is locally free for every integer $b \geq 0$, then the spectral sequence (2.20) degenerates at the $E_{1}$ terms.

Proof. By (4.37), if $R^{b} f_{*}^{\text {tor }}\left(\mathcal{O}_{\mathrm{N}^{\text {tor }}}\right)$ is locally free for every integer $b \geq 0$, then all the $E_{1}$ terms $R^{b} f_{*}^{\text {tor }}\left(\bar{\Omega}_{\mathrm{N}^{\text {tor }} / \mathrm{M}_{\mathcal{H}}^{\text {tor }}}^{a}\right)$ of the spectral sequence $(2.20)$ are locally free. Therefore, to show that (2.20) degenerates at $E_{1}$ terms, it suffices to show that it degenerates at $E_{1}$ terms over the open dense subscheme $\mathrm{M}_{\mathcal{H}}$ of $\mathrm{M}_{\mathcal{H}}^{\text {tor }}$, which is true because $\left.f^{\text {tor }}\right|_{\mathrm{N}}=f: \mathrm{N} \rightarrow \mathrm{M}_{\mathcal{H}}$ is an abelian scheme. (See for example [Berthelot et al. 1982, Proposition 2.5.2].)

This proves (3c) of Theorem 2.15, because the local freeness of $R^{b} f_{*}^{\text {tor }}\left(\mathrm{O}_{\mathrm{N}^{\text {tor }}}\right)$ has been established in Section 4B for every integer $b \geq 0$.

4D. Gauss-Manin connections with log poles. In Section 3C, we proved the log smoothness of $f^{\text {tor }}: \mathrm{N}^{\text {tor }} \rightarrow \mathrm{M}_{\mathcal{H}}^{\text {tor }}$ by verifying Lemma 3.11. For simplicity, let us set

$$
\bar{\Omega}_{\mathrm{M}_{\mathcal{H}}^{\text {tor }} / \mathrm{S}_{0}}^{1}:=\Omega_{\mathrm{M}_{\mathcal{H}}^{\text {tor }} / \mathrm{S}_{0}}^{1}[d \log \infty] \quad \text { and } \quad \bar{\Omega}_{\mathrm{N}^{\text {tor }} / \mathrm{S}_{0}}^{1}:=\Omega_{\mathrm{N}^{\text {tor }} / \mathrm{S}_{0}}^{1}[d \log \infty] .
$$

Then (3.12) can be rewritten as the exact sequence

$$
0 \rightarrow\left(f^{\text {tor }}\right)^{*}\left(\bar{\Omega}_{\mathrm{M}_{\mathcal{H}}^{\text {tor }} / \mathrm{S}_{0}}^{1}\right) \rightarrow \bar{\Omega}_{\mathrm{N}^{\text {tor }} / \mathrm{S}_{0}}^{1} \rightarrow \bar{\Omega}_{\mathrm{N}^{\text {tor }} / \mathrm{M}_{\mathcal{H}}^{\text {tor }}}^{1} \rightarrow 0
$$


which induces the Koszul filtration [Katz 1972, 1.2, 1.3]

$$
\mathrm{K}^{a}\left(\bar{\Omega}_{\mathrm{N}^{\text {tor }} / \mathrm{S}_{0}}\right):=\operatorname{image}\left(\bar{\Omega}_{\mathrm{N}^{\text {tor }} / \mathrm{S}_{0}}^{-a} \otimes_{\mathcal{O}_{\mathrm{N}}}\left(f^{\text {tor }}\right)^{*}\left(\bar{\Omega}_{\mathrm{M}_{\mathcal{H}} / \mathrm{S}_{0}}^{a}\right) \rightarrow \bar{\Omega}_{\mathrm{N}^{\text {tor }} / \mathrm{S}_{0}}\right)
$$

on $\bar{\Omega}_{\mathrm{N}^{\text {tor }} / \mathrm{S}_{0}}^{\cdot}$, with graded pieces $\mathrm{Gr}_{\mathrm{K}}^{a}\left(\bar{\Omega}_{\mathrm{N}^{\text {tor }} / \mathrm{S}_{0}}\right) \cong \bar{\Omega}_{\mathrm{N}^{\text {tor }} / \mathrm{M}_{\mathcal{H}}^{\text {tor }}}^{\bullet-a} \otimes_{\mathcal{O}_{\mathrm{N}}}\left(f^{\text {tor }}\right)^{*}\left(\bar{\Omega}_{\mathrm{M}_{\mathcal{H}}^{\text {tor }} / \mathrm{S}_{0}}^{a}\right)$.

On the other hand, we have the Hodge filtration

$$
\mathrm{F}^{a}\left(\bar{\Omega}_{\mathrm{N}^{\text {tor }} / \mathrm{S}_{0}}\right):=\bar{\Omega}_{\mathrm{N}^{\text {tor }} / \mathrm{S}_{0}}^{: a a}
$$

on $\bar{\Omega}_{\mathrm{N}_{\text {tor }} / \mathrm{S}_{0}}$, giving the Hodge filtration

$$
\mathrm{F}^{a}\left(\underline{H}_{\log -\mathrm{dR}}^{i}\left(\mathrm{~N}^{\text {tor }} / \mathrm{M}_{\mathcal{H}}^{\text {tor }}\right)\right):=\operatorname{image}\left(R^{i} f_{*}^{\text {tor }}\left(\mathrm{F}^{a}\left(\bar{\Omega}_{\mathrm{N}^{\text {tor }} / \mathrm{S}_{0}}\right)\right) \rightarrow R^{i} f_{*}^{\text {tor }}\left(\bar{\Omega}_{\mathrm{N}^{\text {tor }} / \mathrm{S}_{0}}\right)\right)
$$

on $\underline{H}_{\log -\mathrm{dR}}^{i}\left(\mathrm{~N}^{\text {tor }} / \mathrm{M}_{\mathcal{H}}^{\text {tor }}\right)$. By applying $R^{\bullet} f_{*}^{\text {tor }}$ to the short exact sequence

$$
0 \rightarrow \bar{\Omega}_{\mathrm{N}^{\text {tor }} / \mathrm{M}_{\mathcal{H}}^{\text {tor }}}^{\cdot 1} \otimes_{\mathrm{O}_{\mathrm{N}}}\left(f^{\text {tor }}\right)^{*}\left(\bar{\Omega}_{\mathrm{M}_{\mathcal{H}}^{\text {tor }} / \mathrm{S}_{0}}^{1}\right) \rightarrow \mathrm{K}^{2} / \mathrm{K}^{0} \rightarrow \bar{\Omega}_{\mathrm{N}^{\text {tor }} / \mathrm{S}_{0}}^{\cdot} \rightarrow 0,
$$

we obtain in the long exact sequence the connecting homomorphisms

$$
\begin{aligned}
& \underline{H}_{\log -\mathrm{dR}}^{i}\left(\mathrm{~N}^{\text {tor }} / \mathrm{M}_{\mathcal{H}}^{\text {tor }}\right)=R^{i} f_{*}^{\text {tor }}\left(\bar{\Omega}_{\mathrm{N}^{\text {tor }} / \mathrm{M}_{\mathcal{H}}^{\text {tor }}}\right) \\
& \quad \stackrel{\nabla}{\rightarrow} R^{i+1} f_{*}^{\text {tor }}\left(\bar{\Omega}_{\mathrm{N}^{\text {tor }} / \mathrm{M}_{\mathcal{H}}^{\text {tor }}}^{-1} \otimes_{\mathrm{O}_{\mathrm{N}}} \bar{\Omega}_{\mathrm{M}_{\mathcal{H}}^{\text {tor }} / \mathrm{S}_{0}}^{1}\right) \cong \underline{H}_{\log -\mathrm{dR}}^{i}\left(\mathrm{~N}^{\text {tor }} / \mathrm{M}_{\mathcal{H}}^{\text {tor }}\right) \otimes_{\mathrm{O}_{\mathrm{M}_{\mathcal{H}}}} \bar{\Omega}_{\mathrm{M}_{\mathcal{H}}^{\text {tor }} / \mathrm{S}_{0}}^{1} .
\end{aligned}
$$

As explained in [Katz 1972, 1.4], the pullback of $\nabla$ in (4.41) to $\mathrm{M}_{\mathcal{H}}$ is nothing but the usual Gauss-Manin connection on $\underline{H}_{\mathrm{dR}}^{i}\left(\mathrm{~N} / \mathrm{M}_{\mathcal{H}}\right)$. Since the sheaves involved in (4.41) are all locally free,

$$
\nabla: \underline{H}_{\log -\mathrm{dR}}^{i}\left(\mathrm{~N}^{\text {tor }} / \mathrm{M}_{\mathcal{H}}^{\text {tor }}\right) \rightarrow \underline{H}_{\log -\mathrm{dR}}^{i}\left(\mathrm{~N}^{\text {tor }} / \mathrm{M}_{\mathcal{H}}^{\text {tor }}\right) \otimes_{\mathrm{O}_{\mathrm{M}_{\mathcal{H}}}} \bar{\Omega}_{\mathrm{M}_{\mathcal{H}} / \mathrm{S}_{0}}^{1}
$$

satisfies the necessary conditions for being an integrable connection with log poles (because its restriction to the dense subscheme $\mathrm{M}_{\mathcal{H}}$ does). If we take the $\mathrm{F}$-filtration on (4.40), we obtain

$$
0 \rightarrow\left(\mathrm{F}^{a-1}\left(\bar{\Omega}_{\mathrm{N}_{\text {tor }} / \mathrm{M}_{\mathcal{H}}^{\text {tor }}}\right) \otimes_{\mathrm{O}_{\mathrm{N}}}\left(f^{\text {tor }}\right)^{*}\left(\bar{\Omega}_{\mathrm{M}_{\mathcal{H}}^{\text {tor }} / \mathrm{S}_{0}}^{1}\right)\right)[-1] \rightarrow \mathrm{F}^{a}\left(\mathrm{~K}^{2} / \mathrm{K}^{0}\right) \rightarrow \mathrm{F}^{a}\left(\bar{\Omega}_{\mathrm{N}^{\text {tor }} / \mathrm{S}_{0}}\right) \rightarrow 0
$$

and hence the Griffith transversality

$$
\nabla\left(\mathrm{F}^{a}\left(\underline{H}_{\log -\mathrm{dR}}^{i}\left(\mathrm{~N}^{\text {tor }} / \mathrm{M}_{\mathcal{H}}^{\text {tor }}\right)\right)\right) \subset \mathrm{F}^{a-1}\left(\underline{H}_{\log -\mathrm{dR}}^{i}\left(\mathrm{~N}^{\text {tor }} / \mathrm{M}_{\mathcal{H}}^{\text {tor }}\right)\right) \otimes_{\mathrm{O}_{\mathrm{M}_{\mathcal{H}}}} \bar{\Omega}_{\mathrm{M}_{\mathcal{H}}^{\text {tor }} / \mathrm{S}_{0}}^{1}
$$

(as in [Katz 1972, Proposition 1.4.1.6]). This proves (3e) of Theorem 2.15.

Remark 4.42. By (3c) of Theorem 2.15, the (relative) Hodge spectral sequence

$$
E_{1}^{a, i-a}:=R^{i-a} f_{*}^{\text {tor }}\left(\bar{\Omega}_{\mathrm{N}^{\text {tor }} / \mathrm{M}_{\mathcal{H}}^{\text {tor }}}^{a}\right) \Rightarrow \underline{H}_{\log -\mathrm{dR}}^{i}\left(\mathrm{~N}^{\text {tor }} / \mathrm{M}_{\mathcal{H}}^{\text {tor }}\right)
$$

degenerates. Then we have $\operatorname{Gr}_{\mathrm{F}}^{a}\left(\underline{H}_{\log -\mathrm{dR}}^{i}\left(\mathrm{~N}^{\text {tor }} / \mathrm{M}_{\mathcal{H}}^{\text {tor }}\right)\right) \cong R^{i-a} f_{*}^{\text {tor }}\left(\bar{\Omega}_{\mathrm{N}^{\text {tor }} / \mathrm{M}_{\mathcal{H}}^{\text {tor }}}^{a}\right)$, and we can conclude (as in [Katz 1972, Proposition 1.4.1.7]) that the induced morphism 
$\nabla: \mathrm{Gr}_{\mathrm{F}}^{a} \underline{H}_{\log -\mathrm{dR}}^{i}\left(\mathrm{~N}^{\text {tor }} / \mathrm{M}_{\mathcal{H}}^{\text {tor }}\right) \rightarrow \mathrm{Gr}_{\mathrm{F}}^{a-1} \underline{H}_{\log -\mathrm{dR}}^{i}\left(\mathrm{~N}^{\text {tor }} / \mathrm{M}_{\mathcal{H}}^{\text {tor }}\right) \otimes_{\mathrm{O}_{\mathrm{M}_{\mathcal{H}}}} \bar{\Omega}_{\mathrm{M}_{\mathcal{H}}^{\text {tor }} / \mathrm{S}_{0}}^{1}$ agrees with the morphism

$$
R^{i-a} f_{*}^{\text {tor }}\left(\bar{\Omega}_{\mathrm{N}^{\text {tor }} / \mathrm{M}_{\mathcal{H}}^{\text {tor }}}^{a}\right) \rightarrow R^{i-a+1} f_{*}^{\text {tor }}\left(\bar{\Omega}_{\mathrm{N}^{\text {tor }} / \mathrm{M}_{\mathcal{H}}^{\text {tor }}}^{a-1}\right) \otimes_{\mathrm{C}_{\mathrm{M}_{\mathcal{H}}}} \bar{\Omega}_{\mathrm{M}_{\mathcal{H}}^{\text {tor }} / \mathrm{S}_{0}}^{1}
$$

defined by cup product with the Kodaira-Spencer class defined by the extension class of (4.39). We will revisit a special case of this in Section 6B.

\section{Polarizations}

The aim of this section is to prove (3b) and (3d) of Theorem 2.15 , by studying the $\log$ extension of polarizations on the relative de Rham cohomology.

5A. Identification of $\boldsymbol{R}^{\boldsymbol{b}} \boldsymbol{f}_{*}^{\text {tor }}\left(\mathcal{O}_{\mathbf{N}^{\text {tor }}}\right)$. By Corollary 2.12 , any morphism $j_{Q}: Q^{\vee} \hookrightarrow Q$ in Lemma 2.5 (together with the tautological polarization $\lambda_{\mathrm{M}_{\mathcal{H}}}: G_{\mathrm{M}_{\mathcal{H}}} \rightarrow G_{\mathrm{M}_{\mathcal{H}}}^{\vee}$ over $\mathrm{M}_{\mathcal{H}}$ ) induces canonically a polarization

$$
\lambda_{\mathrm{M}_{\mathcal{H}}, j_{Q}}: \underline{\operatorname{Hom}}_{\mathcal{O}}\left(Q, G_{\mathrm{M}_{\mathcal{H}}}\right)^{\circ} \rightarrow\left(\underline{\operatorname{Hom}}_{\mathcal{O}}\left(Q, G_{\mathrm{M}_{\mathcal{H}}}\right)^{\circ}\right)^{\vee}
$$

of degree prime to $\square$, and hence an isomorphism

$$
d \lambda_{\mathrm{M}_{\mathcal{H}}, j_{Q}}: \underline{\operatorname{Hom}}_{\mathcal{O}}\left(Q, \underline{\operatorname{Lie}}_{G_{\mathrm{M}_{\mathcal{H}}} / \mathrm{M}_{\mathcal{H}}}\right) \stackrel{\sim}{\rightarrow} \underline{\operatorname{Hom}}_{\mathcal{O}}\left(Q^{\vee}, \underline{\operatorname{Lie}}_{G_{\mathrm{M}_{\mathcal{H}}}^{\vee} / \mathrm{M}_{\mathcal{H}}}\right) .
$$

Therefore, it induces canonically a $\mathbb{Z}_{(\square)}^{\times}$-polarization $\lambda_{\mathrm{M}_{\mathcal{H}}, j_{Q}}: \mathrm{N} \rightarrow \mathrm{N}^{\vee}$, and hence an isomorphism $d \lambda_{\mathrm{M}_{\mathcal{H}}, j_{Q}}: \underline{\operatorname{Lie}}_{\mathrm{N} / \mathrm{M}_{\mathcal{H}}} \rightarrow \underline{\operatorname{Lie}}_{\mathrm{N}^{\vee} / \mathrm{M}_{\mathcal{H}}}$. Over $\mathrm{M}_{\mathcal{H}}^{\text {tor }}$, the morphisms $j_{Q}: Q^{\vee} \hookrightarrow Q$ and $d \lambda: \underline{\operatorname{Lie}}_{G / \mathrm{M}_{\mathcal{H}}^{\text {tor }}} \rightarrow \underline{\mathrm{Lie}}_{G^{\vee} / \mathrm{M}_{\mathcal{H}}^{\text {tor }}}$ induce canonically an isomorphism $d \lambda_{j_{Q}}: \underline{\operatorname{Hom}}_{\mathcal{O}}\left(Q, \underline{\operatorname{Lie}}_{G / \mathrm{M}_{\mathcal{H}}^{\text {tor }}}\right) \stackrel{\sim}{\longrightarrow} \underline{\operatorname{Hom}}_{\mathcal{O}}\left(Q^{\vee}, \underline{\operatorname{Lie}}_{G^{\vee}} / \mathrm{M}_{\mathcal{H}}^{\text {tor }}\right)$ extending $d \lambda_{\mathrm{M}_{\mathcal{H}}, j_{Q}}: \underline{\operatorname{Hom}}_{\mathcal{O}}\left(Q, \underline{\operatorname{Lie}}_{G_{\mathrm{M}_{\mathcal{H}}} / \mathrm{M}_{\mathcal{H}}}\right) \stackrel{\sim}{\longrightarrow} \underline{\operatorname{Hom}}_{\mathcal{O}}\left(Q^{\vee}, \underline{\operatorname{Lie}}_{G_{\mathrm{M}_{\mathcal{H}}}^{\vee} / \mathrm{M}_{\mathcal{H}}}\right)$.

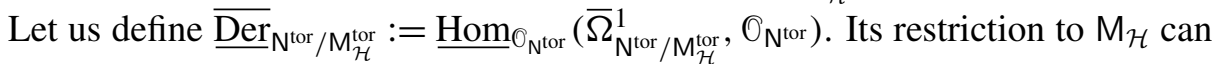
be canonically identified with $\underline{\operatorname{Der}}_{\mathrm{N} / \mathrm{M}_{\mathcal{H}}}:=\underline{\operatorname{Hom}}_{\mathrm{O}_{\mathrm{N}}}\left(\Omega_{\mathrm{N} / \mathrm{M}_{\mathcal{H}}}^{1}, \mathrm{O}_{\mathrm{N}}\right)$.

Let us denote by $\mathrm{J}: \mathrm{M}_{\mathcal{H}} \rightarrow \mathrm{M}_{\mathcal{H}}^{\text {tor }}$ the canonical open immersion. Then we have the commutative diagram

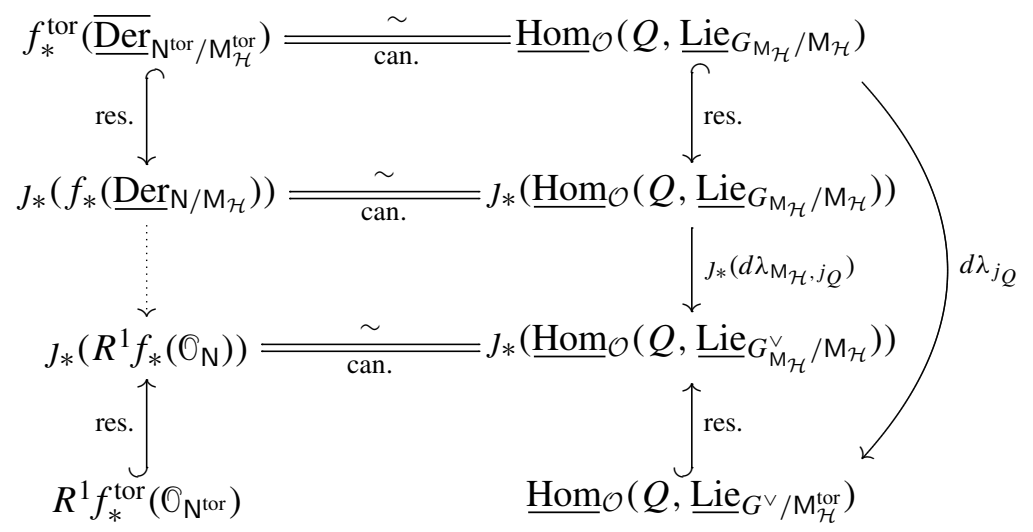


of sheaves over $\mathrm{M}_{\mathcal{H}}^{\text {tor }}$, with the dotted arrow induced by $J_{*}\left(d \lambda_{\mathrm{M}_{\mathcal{H}}, j_{Q}}\right)$. By abuse of notation, let us denote the dotted arrow also by $J_{*}\left(d \lambda_{M_{\mathcal{H}}, j_{Q}}\right)$. We have the following simple observation:

Lemma 5.2. If $J_{*}\left(d \lambda_{\mathrm{M}_{\mathcal{H}}, j_{Q}}\right)$ maps the image of the canonical injection

$$
f_{*}^{\text {tor }}\left(\underline{\underline{\operatorname{Der}}}_{\mathrm{N}^{\text {tor }} / \mathrm{M}_{\mathcal{H}}^{\text {tor }}}\right) \hookrightarrow J_{*}\left(f_{*}\left(\underline{\operatorname{Der}}_{\mathrm{N}} / \mathrm{M}_{\mathcal{H}}\right)\right)
$$

isomorphically to the image of the canonical injection

$$
R^{1} f_{*}^{\text {tor }}\left(\mathcal{O}_{\mathrm{N}^{\text {tor }}}\right) \hookrightarrow J_{*}\left(R^{1} f_{*}\left(\mathrm{O}_{\mathrm{N}}\right)\right),
$$

then (5.1) induces the desired canonical isomorphism

$$
R^{1} f_{*}^{\text {tor }}\left(\mathrm{O}_{\mathrm{N}^{\text {tor }}}\right) \cong \underline{\operatorname{Hom}}_{\mathcal{O}}\left(Q,{\underline{\mathrm{Lie}_{G}}}_{G^{\vee} / \mathrm{M}_{\mathcal{H}}^{\text {tor }}}\right)
$$

extending the canonical isomorphism $R^{1} f_{*}\left(\mathrm{O}_{\mathrm{N}}\right) \cong \underline{\operatorname{Hom}}_{\mathcal{O}}\left(Q, \underline{\operatorname{Lie}}_{G_{\mathrm{M}_{\mathcal{H}}}^{\vee} / \mathrm{M}_{\mathcal{H}}}\right)$ over $\mathrm{M}_{\mathcal{H}}$.

Remark 5.4. The question is whether the assumption of Lemma 5.2 can be satisfied. Since this is a question about morphisms between locally free sheaves over the normal base scheme $\mathrm{M}_{\mathcal{H}}^{\text {tor }}$, it suffices to verify the statement after localizations at points of codimension one. Therefore, since the statement is tautologically true over $M_{\mathcal{H}}$, it suffices to verify it over $M_{\mathcal{H}}^{\text {tor }} \otimes_{\mathbb{Z}} \mathbb{Q}$.

5B. Logarithmic extension of polarizations. By construction (see Section 3A), $\tilde{X}^{\vee}(1) \cong \operatorname{Hom}_{\mathcal{O}}\left(\tilde{X}, \operatorname{Diff}^{-1}(1)\right)$ is the submodule $Q_{-2}$ of $Q^{\vee} \otimes_{\mathbb{Z}} \mathbb{Z}_{(\square)}(1)$, and $\widetilde{Y}$ is the submodule $Q_{0}$ of $Q \otimes_{\mathbb{Z}} \mathbb{Z}_{(\square)}$. Therefore, the embedding $j_{Q}: Q^{\vee} \hookrightarrow Q$ corresponds to an element $\tilde{\ell}_{j_{Q}}$ of $\mathbf{S}_{\widetilde{\Phi}_{\tilde{\mathcal{H}}}} \otimes_{\mathbb{Z}} \mathbb{Z}_{(\square)}$. The positive definiteness of the induced pairing $\left\langle j_{Q}^{-1}(\cdot), \cdot\right\rangle_{Q}$ then translates to the strong positivity condition that $\left\langle\tilde{\ell}_{j_{Q}}, y\right\rangle>0$ for any $y \in \mathbf{P}_{\widetilde{\Phi}_{\tilde{\mathcal{H}}}}-\{0\}$. By replacing $j_{Q}$ with a multiple by a positive integer prime to $\square$, we may and we shall assume that $\tilde{\ell}_{j_{Q}} \in \mathbf{S}_{\widetilde{\Phi}_{\tilde{\mathcal{H}}}}$ (without altering the

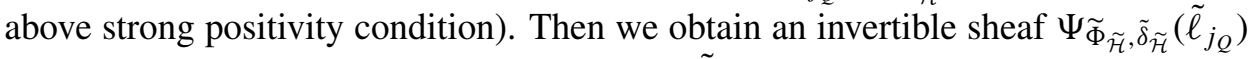
over the abelian scheme $\mathrm{N} \rightarrow \mathrm{M}_{\mathcal{H}}$. Note that $\tilde{\ell}_{j_{Q}} \in \tilde{\sigma}_{0}^{\vee}$.

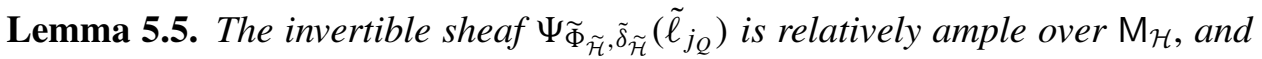

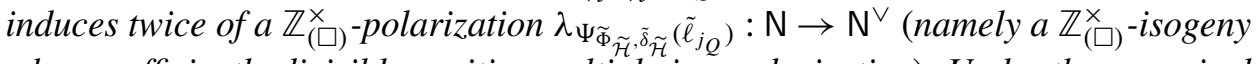
whose sufficiently divisible positive multiple is a polarization). Under the canonical isomorphisms in Corollary 2.13, the induced morphism

$$
d \lambda_{\Psi_{\tilde{\Phi}_{\tilde{\mathcal{H}}}, \tilde{\delta}_{\mathcal{H}}}\left(\tilde{\ell}_{j_{Q}}\right)}: \underline{\operatorname{Lie}_{\mathrm{N}} / \mathrm{M}_{\mathcal{H}}} \rightarrow \underline{\operatorname{Lie}_{N}} \mathrm{~N}^{\vee} \mathrm{M}_{\mathcal{H}}
$$

is twice a positive $\mathbb{Z}_{(\square)}^{\times}$-multiple of

$$
d \lambda_{\mathrm{M}_{\mathcal{H}}, j_{Q}}: \underline{\operatorname{Hom}}_{\mathcal{O}}\left(Q, \underline{\operatorname{Lie}}_{G_{\mathrm{M}_{\mathcal{H}}} / \mathrm{M}_{\mathcal{H}}}\right) \stackrel{\sim}{\rightarrow} \underline{\operatorname{Hom}}_{\mathcal{O}}\left(Q^{\vee}, \underline{\operatorname{Lie}}_{G_{\mathrm{M}_{\mathcal{H}}}^{\vee} / \mathrm{M}_{\mathcal{H}}}\right) .
$$

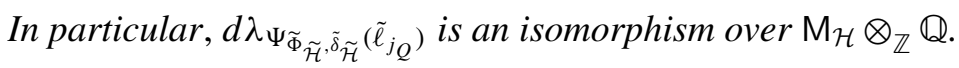




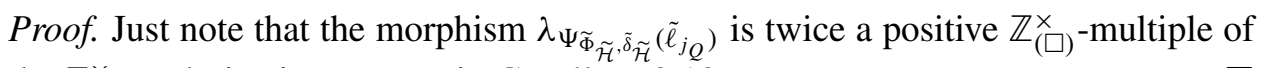
the $\mathbb{Z}_{(\square)}^{\times}$-polarization $\lambda_{\mathrm{M}_{\mathcal{H}}, j_{Q}}$ in Corollary 2.12 .

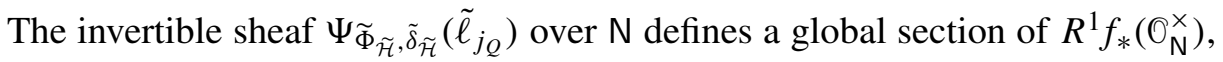
and the morphism

$$
d \log : \mathrm{O}_{\mathrm{N}}^{\times} \rightarrow \Omega_{\mathrm{N} / \mathrm{M}_{\mathcal{H}}}^{1}, \quad a \mapsto a^{-1} d a
$$

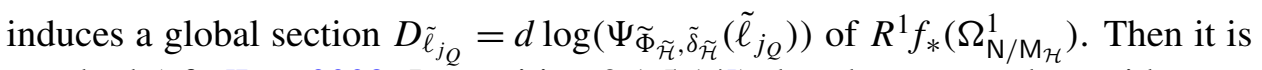
standard (cf. [Lan 2008, Proposition 2.1.5.14]) that the cup product with $D_{\tilde{\ell}_{j_{Q}}}$ induces a composition of morphisms

$$
f_{*}\left(\underline{\operatorname{Der}}{ }_{\mathrm{N} / \mathrm{M}_{\mathcal{H}}}\right) \stackrel{\cup D_{\tilde{\ell}_{j}}}{\rightarrow} R^{1} f_{*}\left(\underline{\operatorname{Der}} \mathrm{N} / \mathrm{M}_{\mathcal{H}} \otimes_{\mathrm{O}_{\mathrm{N}}} \Omega_{\mathrm{N} / \mathrm{M}_{\mathcal{H}}}^{1}\right) \stackrel{\text { can. }}{\rightarrow} R^{1} f_{*}\left(\mathrm{O}_{\mathrm{N}}\right),
$$

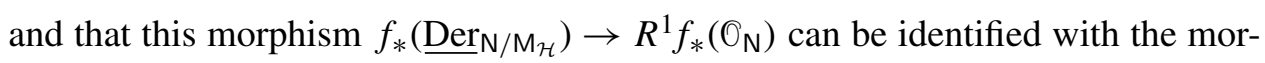

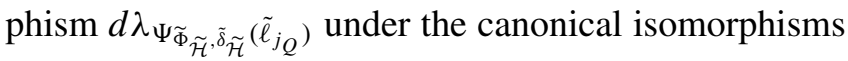

$$
f_{*}\left(\underline{\operatorname{Der}}_{\mathrm{N} / \mathrm{M}_{\mathcal{H}}}\right) \cong \underline{\operatorname{Lie}}_{\mathrm{N}} / \mathrm{M}_{\mathcal{H}} \quad \text { and } \quad R^{1} f_{*}\left(\mathrm{O}_{\mathrm{N}}\right) \cong \underline{\operatorname{Lie}}_{\mathrm{N}}{ } / \mathrm{M}_{\mathcal{H}} .
$$

The first question is whether we can extend the morphism $f_{*}\left(\underline{\operatorname{Der}} \mathrm{N} / \mathrm{M}_{\mathcal{H}}\right) \rightarrow R^{1} f_{*}\left(\mathrm{O}_{\mathrm{N}}\right)$ to $\mathrm{M}_{\mathcal{H}}^{\text {tor }}$; and the second question is whether the extended morphism is an isomorphism, at least in codimension one.

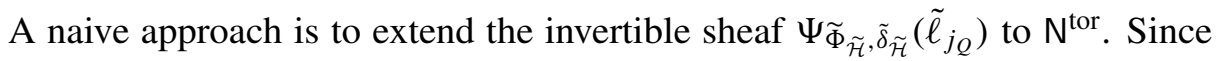
$\mathrm{N}^{\text {tor }}$ is projective and smooth over $\mathrm{S}_{0}=\operatorname{Spec}\left(\mathcal{O}_{F_{0},(\square)}\right)$, it is locally noetherian and locally factorial. Then [EGA $\mathrm{IV}_{4}$ 1967, 21.6.11] implies that the canonical restriction morphism $\operatorname{Pic}\left(\mathrm{N}^{\text {tor }}\right) \rightarrow \operatorname{Pic}(\mathrm{N})$ is surjective.

However, since $f^{\text {tor }}: \mathrm{N}^{\text {tor }} \rightarrow \mathrm{M}_{\mathcal{H}}^{\text {tor }}$ is not smooth, we have little control on the canonical restriction morphism $R^{1} f_{*}^{\text {tor }}\left(\Omega_{\mathrm{N}^{\text {tor }} / \mathrm{M}_{\mathcal{H}}^{\text {tor }}}\right) \stackrel{\text { res. }}{\rightarrow} J_{*}\left(R^{1} f_{*}\left(\Omega_{\mathrm{N} / \mathrm{M}_{\mathcal{H}}}^{1}\right)\right.$ ), and there is no obvious reason that the image of the class defined by any extension of

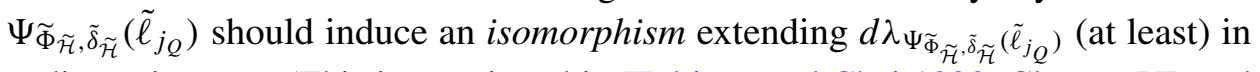
codimension one. (This is mentioned in [Faltings and Chai 1990, Chapter VI, end of $\$ 2]$, but with no details.)

An alternative approach is to consider the canonical restriction morphism

$$
R^{1} f_{*}^{\text {tor }}\left(\bar{\Omega}_{\mathrm{N}^{\text {tor }} / \mathrm{M}_{\mathcal{H}}^{\text {tor }}}\right) \stackrel{\text { res. }}{\rightarrow} J_{*}\left(R^{1} f_{*}\left(\Omega_{\mathrm{N} / \mathrm{M}_{\mathcal{H}}}^{1}\right)\right)
$$

By Lemma 4.29, and by (3a) of Theorem $2.15, R^{1} f_{*}^{\text {tor }}\left(\bar{\Omega}_{\mathrm{N}^{\text {tor }} / \mathrm{M}_{\mathcal{H}}^{\text {tor }}}\right)$ is locally free over $\mathrm{M}_{\mathcal{H}}^{\text {tor }}$. Therefore, the morphism (5.6) is injective.

Remark 5.7. The use of $R^{1} f_{*}^{\text {tor }}\left(\bar{\Omega}_{\mathrm{N}^{\text {tor }} / \mathrm{M}_{\mathcal{H}}^{\text {tor }}}\right)$ is inspired by Kato's idea of (relative) log Picard groups mentioned in [Illusie 1994, 3.3]. An application of this idea has been carried out in [Olsson 2004]. 
So far we have refrained from introducing the log structures (because they had not been necessary), but they are needed (at least formally) here. We shall adopt a notation slightly different from those of [Kato 1989; Illusie 1994]. Let $\breve{J}: \mathrm{N} \rightarrow \mathrm{N}^{\text {tor }}$ denote the canonical open immersion. Then the canonical (fine) $\log$ structure on $\mathrm{N}^{\text {tor }}$ (which we have been using so far) given by $\mathrm{N}^{\text {tor }}-\mathrm{N}$ (with its reduced structure) can be defined explicitly as the sheaf of monoids $\overline{\mathrm{O}}_{\mathrm{N}^{\text {tor }}}^{\times}:=\mathrm{O}_{\mathrm{N} \text { tor }} \cap \breve{\jmath}_{*} \mathrm{O}_{\mathrm{N}}^{\times}$(sheafification of the obvious presheaf), with associated sheaf of groups $\overline{\mathrm{O}}_{\mathrm{N}}^{\times \text {tor }}$. Clearly, the restriction of $\overline{\mathrm{O}}_{\mathrm{N}}^{\times, \mathrm{gp}}$ to $\mathrm{N}$ is canonically isomorphic to $\mathrm{O}_{\mathrm{N}}^{\times}$.

Definition 5.8. A relative $\log$ invertible sheaf over $f^{\text {tor }}: \mathrm{N}^{\text {tor }} \rightarrow \mathrm{M}_{\mathcal{H}}^{\text {tor }}$ is a global section of $R^{1} f_{*}^{\text {tor }}\left(\overline{\mathrm{O}}_{\mathrm{N}}^{\times \text {tor }}\right)$.

Since we do not assume that $f^{\text {tor }}$ is flat (or log integral), the appropriate interpretation of relative log invertible sheaves can be quite delicate (and beyond this article).

Lemma 5.9. To define a global section of $R^{1} f_{*}^{\text {tor }}\left(\overline{\mathrm{O}}_{\mathrm{N}}^{\times, \mathrm{gp}}\right)$, it suffices to have the following data:

(1) A collection of schemes $\bar{U}_{\alpha}$ over $\mathrm{N}^{\text {tor }}$ forming an étale covering. We shall denote the fiber product $\bar{U}_{\alpha} \times_{\mathrm{N}^{\text {tor }}} \bar{U}_{\beta}$ (i.e., "intersection" in the étale topology) by $\bar{U}_{\alpha \beta}$, denote $\left.\bar{U}_{\alpha \beta}\right|_{\mathrm{N}}:=\bar{U}_{\alpha \beta} \times_{\mathrm{N}^{\text {tor }}} \mathrm{N}$ by $U_{\alpha \beta}$, and use similar notations for higher fiber products.

(2) A usual invertible sheaf $\mathcal{L}_{\alpha}$ over each $\bar{U}_{\alpha}$.

(3) A comparison isomorphism $\left.\left.\mathcal{L}_{\alpha}\right|_{U_{\alpha \beta}} \cong \mathcal{L}_{\beta}\right|_{U_{\alpha \beta}}$ over each $U_{\alpha \beta}$, satisfying the usual cocycle condition over triple fiber products $U_{\alpha \beta \gamma}$.

Proof. Since the restriction morphism $\overline{\mathrm{O}}_{\mathrm{N}^{\text {tor }}}^{\times \mathrm{gp}}\left(\bar{U}_{\alpha \beta}\right) \rightarrow \overline{\mathrm{O}}_{\mathrm{N}^{\text {tor }}}^{\times \mathrm{gp}}\left(U_{\alpha \beta}\right) \cong \mathcal{O}_{\mathrm{N}}^{\times}\left(U_{\alpha \beta}\right)$ is a bijection when the image of $\bar{U}_{\alpha \beta}$ in $\mathrm{N}^{\text {tor }}$ is sufficiently small, the data above define a section of $H^{1}\left(\mathrm{~N}^{\text {tor }}, \overline{\mathrm{O}}_{\mathrm{N}^{\text {tor }}}^{\times, \mathrm{gp}}\right)$, which then defines a section of $H^{0}\left(\mathrm{M}_{\mathcal{H}}^{\text {tor }}, R^{1} f_{*}^{\text {tor }}\left(\overline{\mathrm{O}}_{\mathrm{N}^{\text {tor }}}^{\times, \mathrm{gp}}\right)\right)$ by the Leray spectral sequence in low degrees. (See [Godement 1958, I 4.5.1].)

In the construction of toroidal compactifications in [Lan 2008, §6.3.3] (following [Faltings and Chai 1990, Chapter IV, §5]), there is a strata-preserving étale covering $\widetilde{U} \rightarrow \widetilde{M}_{\widetilde{\mathcal{H}}}^{\text {tor }}$ (serving as an étale presentation for the algebraic stack $\widetilde{M}_{\widetilde{\mathcal{H}}}^{\text {tor }}$ ), where $\widetilde{U}$ is a finite union of the so-called good algebraic models of $\widetilde{\mathrm{M}}_{\tilde{\mathcal{H}}}^{\text {tor }}$. (See [Lan 2008, Definition 6.3.2.5].) By taking the closures of the $\left[\left(\widetilde{\Phi}_{\widetilde{\mathcal{H}}}, \tilde{\delta}_{\widetilde{\mathcal{H}}}, \widetilde{\sigma}\right)\right]$-strata, we obtain a strata-preserving étale covering $\breve{\mathrm{U}} \rightarrow \mathrm{N}^{\text {tor }}$, with strata labeled by triples $\left[\left(\breve{\Phi}_{\widetilde{\mathcal{H}}}, \breve{\delta}_{\widetilde{\mathcal{H}}}, \breve{\tau}\right)\right]$ having $\left[\left(\widetilde{\Phi}_{\widetilde{\mathcal{H}}}, \tilde{\delta}_{\widetilde{\mathcal{H}}}, \widetilde{\sigma}\right)\right]$ as a face.

Each connected component $\bar{U}_{\alpha}$ of $\breve{U}$ is given by the closure of the $\left[\left(\widetilde{\Phi}_{\widetilde{\mathcal{H}}}, \tilde{\delta}_{\widetilde{\mathcal{H}}}, \widetilde{\sigma}\right)\right]$ stratum in a so-called good algebraic $\left(\breve{\Phi}_{\widetilde{\mathcal{H}}}, \breve{\delta}_{\widetilde{\mathcal{H}}}, \breve{\tau}\right)$-model $\widetilde{U}_{\alpha}=\operatorname{Spec}\left(\widetilde{R}_{\alpha}\right) \rightarrow \widetilde{\mathrm{M}}_{\widetilde{\mathcal{H}}}^{\text {tor }}$, where $\left(\breve{\Phi}_{\widetilde{\mathcal{H}}}, \breve{\delta}_{\widetilde{\mathcal{H}}}, \breve{\tau}\right)$ is a representative of some $\left[\left(\breve{\Phi}_{\widetilde{\mathcal{H}}}, \breve{\delta}_{\widetilde{\mathcal{H}}}, \breve{\tau}\right)\right]$ having $\left[\left(\widetilde{\Phi}_{\widetilde{\mathcal{H}}}, \tilde{\delta}_{\widetilde{\mathcal{H}}}, \widetilde{\sigma}\right)\right]$ 
as a face (cf. second property in [Lan 2008, Definition 6.3.2.5]), which we may assume to satisfy $\breve{\tau} \in \Sigma_{\breve{\Phi}_{\breve{H}}, \breve{\sigma}, \tau}$. (See Section 4A. There are usually many $\alpha$ for each $\left[\left(\breve{\Phi}_{\widetilde{\mathcal{H}}}, \breve{\delta}_{\widetilde{\mathcal{H}}}, \breve{\tau}\right)\right]$.) Then we also have a strata-preserving étale morphism $\bar{U}_{\alpha} \rightarrow$ $\left(\widetilde{\Xi}_{\breve{\Phi}_{\tilde{\mathcal{H}}}, \breve{\delta}_{\tilde{\mathcal{H}}}}\right)_{\breve{\sigma}}(\breve{\tau})$, which we shall call a good algebraic $\left(\breve{\Phi}_{\widetilde{\mathcal{H}}}, \breve{\delta}_{\widetilde{\mathcal{H}}}, \breve{\tau}\right)$-model of $\mathrm{N}^{\text {tor }}$. The (open) $\left[\left(\widetilde{\Phi}_{\widetilde{\mathcal{H}}}, \tilde{\delta}_{\widetilde{\mathcal{H}}}, \widetilde{\sigma}\right)\right]$-stratum in $\bar{U}_{\alpha}$ is exactly the open subscheme $U_{\alpha}:=\bar{U}_{\alpha} \times{ }_{\mathrm{N}}$ tor $\mathrm{N}$ of $\bar{U}_{\alpha}$.

Lemma 5.10. Suppose that, for each $\breve{\tau} \in \Sigma_{\breve{\Phi}_{\tilde{\mathcal{H}}}, \breve{\sigma}, \tau}$, we have chosen an element $\breve{\ell}_{j_{Q}, \breve{\tau}}$ in $\breve{\tau}_{0}^{\vee}$ that is mapped to $\tilde{\ell}_{j_{Q}}$ in $\widetilde{\sigma}_{0}^{\vee}$ under the second morphism in (3.6), and that $\breve{\ell}_{j_{Q}, \gamma \bar{\tau}}=\gamma \breve{\ell}_{j_{Q}, \breve{\tau}}$ for any $\gamma \in \Gamma_{\widetilde{\Phi}_{\tilde{H}}, \Phi_{\mathcal{H}}}$. (Note that the choice of $\breve{\ell}_{j_{Q}, \breve{\tau}}$ is unique only up to translation by $\breve{\sigma}^{\perp}$.) Let $\breve{\mathrm{U}} \rightarrow \mathrm{N}^{\text {tor }}$ be any strata-preserving étale covering formed

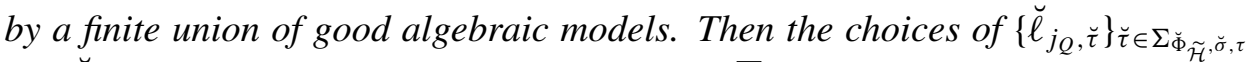
and $\breve{\mathrm{U}}$ determine a relative log invertible sheaf $\overline{\mathcal{L}}$ over $\mathrm{N}^{\text {tor }} \rightarrow \mathrm{M}_{\mathcal{H}}^{\text {tor }}$ extending the

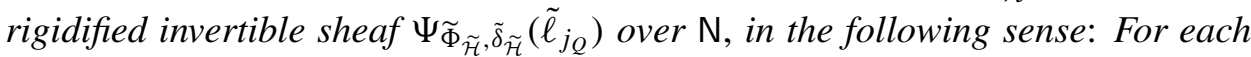
good algebraic $\left(\breve{\Phi}_{\widetilde{\mathcal{H}}}, \breve{\delta}_{\widetilde{\mathcal{H}}}, \breve{\tau}\right)$-model $\bar{U}_{\alpha}$ of $\mathrm{N}^{\text {tor }}$, with $\breve{\tau} \in \Sigma_{\breve{\Phi}_{\tilde{\mathcal{H}}}, \breve{\sigma}, \tau}$, let $\mathcal{L}_{\alpha}$ denote the

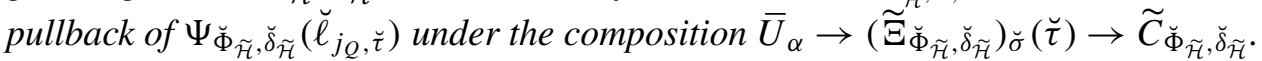
Then $\left.\mathcal{L}_{\alpha}\right|_{U_{\alpha}}$ is canonically isomorphic to the pullback of $\Psi_{\widetilde{\Phi}_{\widetilde{\mathcal{H}}}, \tilde{\delta}_{\widetilde{\mathcal{H}}}}\left(\tilde{\ell}_{j_{Q}}\right)$ (from $\mathrm{N} \cong$ $\left.\widetilde{C}_{\widetilde{\Phi}_{\tilde{\mathcal{H}}}, \tilde{\delta}_{\widetilde{\mathcal{H}}}}\right)$ to $U_{\alpha}$. Furthermore, the collection $\left\{\left(U_{\alpha}, \mathcal{L}_{\alpha}\right)\right\}$ satisfies the requirements in Lemma 5.9, and defines a log invertible sheaf as in Definition 5.8.

Proof. Let $\left(\widetilde{G}, \tilde{\lambda}, \tilde{i}, \widetilde{\alpha}_{\widetilde{\mathcal{H}}}\right)$ be the degenerating family of type $\widetilde{\mathrm{M}}_{\widetilde{\mathcal{H}}}$ over $\widetilde{\mathrm{M}}_{\widetilde{\mathcal{H}}}^{\text {tor }}$. Let $\underline{B}(\widetilde{G}): \underline{\mathbf{S}}_{\widetilde{\Phi}_{\widetilde{\mathcal{H}}}(\widetilde{G})} \rightarrow \underline{\operatorname{Inv}}\left(\widetilde{\mathrm{M}}_{\widetilde{\mathcal{H}}}^{\text {tor }}\right)$ be constructed as in [Lan 2008, Construction 6.3.1.1]. If $\widetilde{U}_{\alpha}$ is a good algebraic $\left(\breve{\Phi}_{\widetilde{\mathcal{H}}}, \breve{\delta}_{\widetilde{\mathcal{H}}}, \breve{\tau}\right)$-model, then for any $\breve{\ell} \in \mathbf{S}_{\breve{\Phi}_{\widetilde{\mathcal{H}}}}$, the invertible sheaf $\underline{B}(\widetilde{G})\left(\widetilde{U}_{\alpha}\right)(\breve{\ell})$ over $\widetilde{U}_{\alpha}$ is canonically isomorphic to the pullback of $\Psi_{\breve{\Phi}_{\widetilde{\mathcal{H}}}, \breve{\delta}_{\tilde{\mathcal{H}}}}(\breve{\ell})$ under the composition $\widetilde{U}_{\alpha} \rightarrow\left(\widetilde{\Xi}_{\breve{\Phi}_{\tilde{\mathcal{H}}}, \breve{\delta}_{\tilde{\mathcal{H}}}}\right) \breve{\sigma}(\breve{\tau}) \rightarrow \widetilde{C}_{\breve{\Phi}_{\tilde{\mathcal{H}}}, \breve{\delta}_{\tilde{\mathcal{H}}}}$ (cf. third property in [Lan 2008, Definition 6.3.2.5]).

Given that $\underline{B}(\widetilde{G})$ is defined over $\widetilde{\mathrm{M}}_{\widetilde{\mathcal{H}}}^{\text {tor }}$ and functorial with respect to pullback

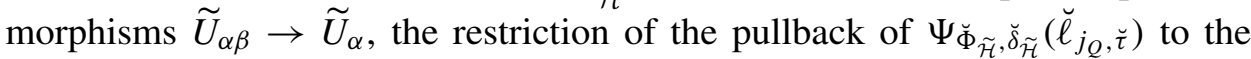

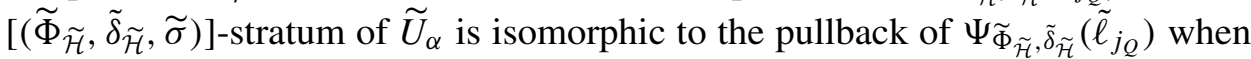
$\left(\widetilde{\Phi}_{\widetilde{\mathcal{H}}}, \tilde{\delta}_{\widetilde{\mathcal{H}}}, \widetilde{\sigma}\right)$ is a face of $\left[\left(\breve{\Phi}_{\widetilde{\mathcal{H}}}, \breve{\delta}_{\widetilde{\mathcal{H}}}, \breve{\tau}\right)\right]$. In other words, $\left.\mathcal{L}_{\alpha}\right|_{U_{\alpha}}$ is isomorphic to the

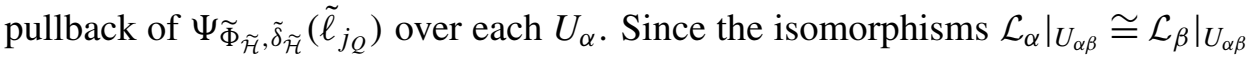

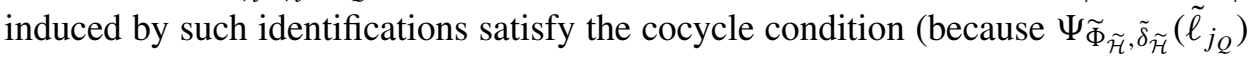
is defined on $\mathrm{N}$ ), the claim follows, as desired.

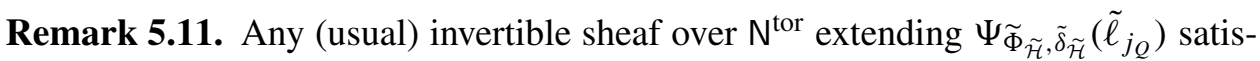
fies the requirements in Lemma 5.9 trivially. The point of Lemma 5.10 is that it

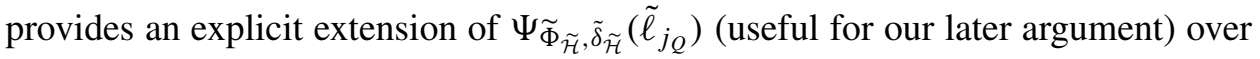
an étale covering of $\mathrm{N}^{\text {tor }}$. (We do not have such an explicit description of a global invertible sheaf extension over $\mathrm{N}^{\text {tor }}$.) 
Definition 5.12. To any relative log invertible sheaf $\overline{\mathcal{L}}$ over $\mathrm{N}^{\text {tor }} \rightarrow \mathrm{M}_{\mathcal{H}}^{\text {tor }}$ defined by a global section of $R^{1} f_{*}^{\text {tor }}\left(\overline{\mathrm{O}}_{\mathrm{N}_{\text {tor }} \times \text {,g }}\right)$, we define $d \log (\overline{\mathcal{L}})$ to be the image of $\overline{\mathcal{L}}$ under the canonical morphism $R^{1} f_{*}^{\text {tor }}\left(\overline{\mathscr{O}}_{\mathrm{N}^{\text {tor }}}^{\times, \mathrm{gp}}\right) \rightarrow R^{1} f_{*}^{\text {tor }}\left(\bar{\Omega}_{\mathrm{N}^{\text {tor }} / \mathrm{M}_{\mathcal{H}}^{\text {tor }}}^{1}\right)$ induced by the canonical morphism $d \log : \overline{\boldsymbol{O}}_{\mathrm{N}}^{\times, \mathrm{gp}} \rightarrow \bar{\Omega}_{\mathrm{N}^{\text {tor }} / \mathrm{M}_{\mathcal{H}}^{\text {tor }}}^{1}$

Corollary 5.13. There exists a (unique) global section $D_{\tilde{\ell}_{Q}}^{\text {tor }}$ of $R^{1} f_{*}^{\text {tor }}\left(\bar{\Omega}_{\mathrm{N}^{\text {tor }} / \mathrm{M}_{\mathcal{H}}^{\text {tor }}}^{1}\right)$ whose image under the canonical injection (5.6) is $J_{*}\left(D_{\tilde{\ell}_{j_{Q}}}\right)$, and which satisfies $D_{\tilde{\ell}_{j_{Q}}}^{\text {tor }}=d \log (\overline{\mathcal{L}})$ for any $\overline{\mathcal{L}}$ constructed in Lemma 5.10 (with any choices of $\breve{\ell}_{j_{Q}, \breve{\tau}}{ }^{\prime} s$ ).

Proof. Existence is clear because there is always some (usual) invertible sheaf

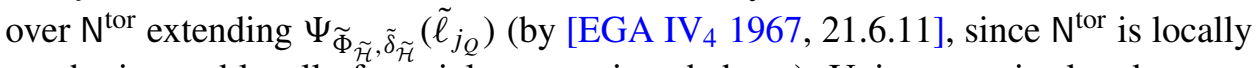
noetherian and locally factorial, as mentioned above). Uniqueness is clear because (5.6) is injective. Once we know the unique existence of $D_{\tilde{\ell}_{j_{Q}}}^{\text {tor }}$, it has to agree with $d \log (\overline{\mathcal{L}})$ for any $\overline{\mathcal{L}}$ constructed in Lemma 5.10.

Thus we are led to state the following:

Proposition 5.14. Cup product with the global section $D_{\tilde{\ell}_{\mathcal{Q}}}^{\text {tor }}$ of $R^{1} f_{*}^{\text {tor }}\left(\bar{\Omega}_{\mathrm{N}^{\text {tor }} / \mathrm{M}_{\mathcal{H}}^{\text {tor }}}^{1}\right)$ in Corollary 5.13 induces a composition of morphisms

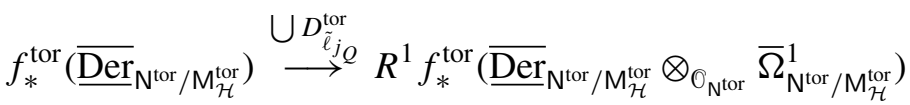

$$
\begin{aligned}
& \stackrel{\text { can. }}{\longrightarrow} R^{1} f_{*}^{\text {tor }}\left(\mathrm{O}_{\mathrm{N}^{\text {tor }}}\right) \text {. }
\end{aligned}
$$

This composition is an isomorphism over $\mathrm{M}_{\mathcal{H}}^{\text {tor }} \otimes_{\mathbb{Z}} \mathbb{Q}$. (By Lemma 5.2 and Remark 5.4, this implies the existence of the canonical isomorphism (5.3).)

We will carry out the proof of Proposition 5.14 in the next subsection.

5C. Induced morphisms over formal fibers. We fix the choices of $\left\{\breve{\ell}_{j_{Q}, \breve{\tau}}\right\}_{\breve{\tau} \in \Sigma_{\Phi_{\tilde{\mathcal{H}}}, \check{\sigma}, \tau}}$ and $\breve{U}$, so that $\overline{\mathcal{L}}$ is constructed as in Lemma 5.10, and so that $D_{\tilde{\ell}_{j_{Q}}}^{\text {tor }}=d \log (\overline{\mathcal{L}})$ as in Corollary 5.13 .

Since $f^{\text {tor }}$ is proper and the sheaves involved are all coherent, by Grothendieck's fundamental theorem [EGA III ${ }_{1}$ 1961, 4.1.5], Proposition 5.14 can be verified by pulling back to formal completions along strata of $\mathrm{M}_{\mathcal{H}}^{\text {tor }}$. Let us fix the choice of a cusp label $\left[\left(\Phi_{\mathcal{H}}, \delta_{\mathcal{H}}, \sigma\right)\right]$ of $\mathrm{M}_{\mathcal{H}}^{\text {tor }}$, and consider the canonical morphism

$$
\imath: \mathfrak{X}_{\Phi_{\mathcal{H}}, \delta_{\mathcal{H}}, \tau} \cong\left(\mathrm{M}_{\mathcal{H}}^{\text {tor }}\right)_{\mathrm{Z}_{\left[\left(\Phi_{\mathcal{H}}, \delta_{\mathcal{H}}, \sigma\right)\right]}} \rightarrow \mathrm{M}_{\mathcal{H}}^{\text {tor }}
$$

By abuse of notation, we shall also denote by $\imath^{*}(\cdot)$ the pullbacks of objects under pullbacks of the morphism $l$. We would like to show that the morphism $\imath^{*} f_{*}^{\text {tor }}\left(\overline{\operatorname{Der}}_{\mathrm{N}_{\text {tor }} / \mathrm{M}_{\mathcal{H}}^{\text {tor }}}\right) \rightarrow \imath^{*} R^{1} f_{*}^{\text {tor }}\left(\mathcal{O}_{\mathrm{N}^{\text {tor }}}\right)$ defined by cup product with $\imath^{*}\left(D_{\tilde{\ell}_{\mathcal{Q}}}^{\text {tor }}\right)$ is an isomorphism over $\mathfrak{X}_{\Phi_{\mathcal{H}}, \delta_{\mathcal{H}}, \tau} \otimes_{\mathbb{Z}} \mathbb{Q}$. 
As said in Section 4A, the pullback of $f^{\text {tor }}$ to $\mathfrak{X}_{\Phi_{\mathcal{H}}, \delta_{\mathcal{H}}, \tau}$ can be identified with the

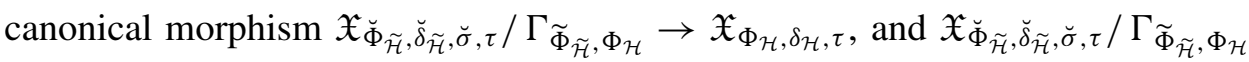

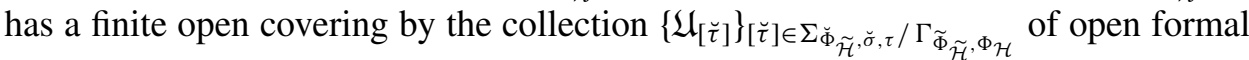
subschemes. Let $\breve{\tau} \in \Sigma_{\breve{\Phi}_{\tilde{\mathcal{H}}}, \breve{\sigma}, \tau}$ be a representative of $[\breve{\tau}] \in \Sigma_{\breve{\Phi}_{\tilde{\mathcal{H}}}, \breve{\sigma}, \tau} / \Gamma_{\widetilde{\Phi}_{\tilde{\mathcal{H}}}, \Phi_{\mathcal{H}}}$. For each such $\breve{\tau}$, recall that the formal scheme $\mathfrak{U}_{\breve{\tau}}$ is the completion of $\left(\widetilde{\Xi}_{\left.\breve{\Phi}_{\widetilde{\mathcal{H}}}, \breve{\delta}_{\widetilde{\mathcal{H}}}\right)}\right)_{\tilde{\sigma}}(\breve{\tau})$ along $U_{\breve{\tau}}$.

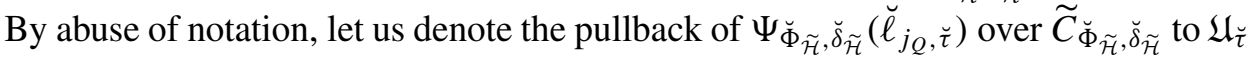
by the same notation. For any $\gamma \in \Gamma_{\widetilde{\Phi}_{\tilde{\mathcal{H}}}, \Phi_{\mathcal{H}}}$, since $\breve{\ell}_{j_{Q}, \gamma \breve{\tau}}=\gamma \breve{\ell}_{j_{Q}, \breve{\tau}}$ (see Lemma 5.10),

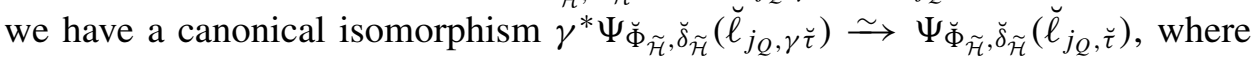
$\gamma: \mathfrak{U}_{\breve{\tau}} \stackrel{\sim}{\longrightarrow} \mathfrak{U}_{\gamma \breve{\tau}}$ is the canonical isomorphism (see Lemma 4.6). Hence $\left.\Psi_{\breve{\Phi}_{\tilde{\mathcal{H}}}, \breve{\delta}_{\tilde{\mathcal{H}}}} \breve{\ell}_{j_{Q}, \breve{\tau}}\right)$

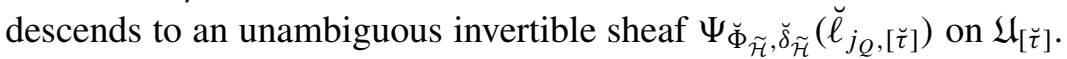

The étale covering $\breve{\mathrm{U}} \rightarrow \mathrm{N}^{\text {tor }}$ induces (by taking formal completion along the pullback of $\left.Z_{\left[\left(\Phi_{\mathcal{H}}, \delta_{\mathcal{H}}, \sigma\right)\right]}\right)$ a formally étale covering of $\left(\mathrm{N}^{\text {tor }}\right)_{\mathrm{Z}_{\left[\left(\Phi_{\mathcal{H}}, \delta_{\mathcal{H}}, \sigma\right)\right]}}$. If $\bar{U}_{\alpha}$ is a good algebraic $\left(\breve{\Phi}_{\widetilde{\mathcal{H}}}, \breve{\delta}_{\widetilde{\mathcal{H}}}, \breve{\tau}\right)$-model of $\mathrm{N}^{\text {tor }}$, then the formal completion $\left(\bar{U}_{\alpha}\right)_{\mathrm{Z}_{\left[\left(\Phi_{\mathcal{H}}, \delta_{\mathcal{H}}, \sigma\right)\right]}}$ of $\bar{U}_{\alpha}$ along the pullback of $Z_{\left[\left(\Phi_{\mathcal{H}}, \delta_{\mathcal{H}}, \sigma\right)\right]}$ is formally étale over $\mathfrak{U}_{\breve{\tau}}$.

Lemma 5.16. The pullback of $\mathcal{L}_{\alpha}$ to $\left(\bar{U}_{\alpha}\right)_{\mathrm{Z}_{\left[\left(\Phi_{\mathcal{H}}, \mathcal{S}_{\mathcal{H}}, \sigma\right)\right]}}$ is isomorphic to the pullback

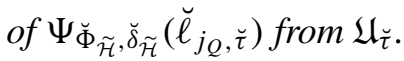

Proof. The canonical morphisms

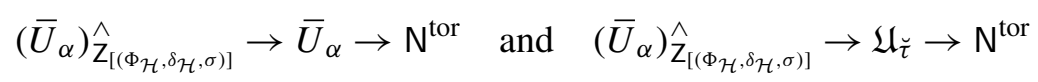

are induced respectively by morphisms

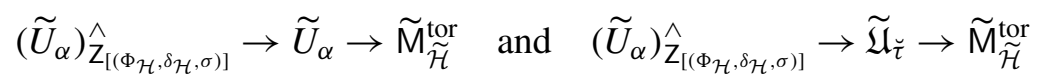

over $\widetilde{\mathrm{M}}_{\widetilde{\mathcal{H}}}^{\text {tor }}$. Under both these morphisms, the pullback of $\left(\widetilde{G}, \widetilde{\lambda}, \tilde{i}, \widetilde{\alpha}_{\widetilde{\mathcal{H}}}\right) \rightarrow \widetilde{\mathrm{M}}_{\widetilde{\mathcal{H}}}^{\text {tor }}$ is canonically isomorphic to the pullback of the Mumford family (as in the proof of Proposition 4.3). Since the isomorphism class of the pullback of $\mathcal{L}_{\alpha}$ to $\left(\bar{U}_{\alpha}\right)_{\mathbf{Z}_{\left[\left(\Phi_{\mathcal{H}}, \delta \mathcal{H}, \sigma\right]\right]}}$ is determined by the pullback of $\underline{B}(\widetilde{G}): \underline{\mathbf{S}}_{\widetilde{\Phi}_{\widetilde{\mathcal{H}}}(\widetilde{G})} \rightarrow \underline{\operatorname{Inv}}\left(\widetilde{\mathrm{M}}_{\widetilde{\mathcal{H}}^{\text {tor }}}^{\text {tor }}\right)$ (as in the proof of Lemma 5.10), we can pullback along $\left(\bar{U}_{\alpha}\right)_{\hat{Z}_{\left[\left(\Phi_{\mathcal{H}}, \delta_{\mathcal{H}}, \sigma\right)\right]}} \rightarrow \mathfrak{U}_{\bar{\tau}} \rightarrow \mathrm{N}^{\text {tor }}$ and conclude that $\mathcal{L}_{\alpha}$ is isomorphic to the pullback of $\Psi_{\overleftarrow{\Phi}_{\tilde{\mathcal{H}}}, \breve{\delta}_{\tilde{\mathcal{H}}}}\left(\ell_{j_{Q}, \breve{\tau}}\right)$ from $\mathfrak{U}_{\breve{\tau}}$.

By Lemma 4.29, we have

$$
l^{*} f_{*}^{\text {tor }}\left(\mathcal{O}_{\mathrm{N}^{\text {tor }}}\right) \cong f_{*}^{\text {tor }}\left(\mathcal{O}_{\left(\mathrm{N}^{\text {tor }}\right)} \hat{\tilde{\mathrm{Z}}}_{\left[\left(\Phi_{\mathcal{H}}, \delta_{\mathcal{H}}, \tau\right)\right]}\right) \cong H^{0}\left(\mathfrak{N}_{\breve{\sigma}, \tau}, \underline{\mathscr{H}}^{0}\left(\mathcal{O}_{\left(\mathrm{N}^{\text {tor }}\right)} \hat{\tilde{\mathrm{Z}}}_{\left[\left(\Phi_{\mathcal{H}}, \delta_{\mathcal{H}}, \tau\right)\right]}\right)\right),
$$

and $\iota^{*} R^{1} f_{*}^{\text {tor }}\left(\mathscr{O}_{\mathrm{N}^{\text {tor }}}\right) \cong R^{1} f_{*}^{\text {tor }}\left(\mathbb{O}_{\left(\mathrm{N}^{\text {tor }}\right)} \hat{\tilde{\mathrm{Z}}}_{\left[\left(\Phi_{\mathcal{H}}, \delta_{\mathcal{H}}, \tau\right)\right]}\right)$ is equipped with a decreasing filtration with (locally free) graded pieces

and

$$
\operatorname{Gr}^{0}\left(\iota^{*} R^{1} f_{*}^{\text {tor }}\left(\mathcal{O}_{\mathrm{N}^{\text {tor }}}\right)\right) \cong H^{0}\left(\mathfrak{N}_{\breve{\sigma}, \tau}, \underline{\mathscr{H}}^{1}\left(\mathrm{O}_{\left(\mathrm{N}^{\mathrm{tor}}\right)} \hat{\tilde{\mathrm{z}}}_{\left[\left(\Phi_{\mathcal{H}}, \delta \mathcal{H}, \tau\right)\right]}\right)\right)
$$

$$
\operatorname{Gr}^{1}\left(\imath^{*} R^{1} f_{*}^{\text {tor }}\left(\mathbb{O}_{\mathrm{N}^{\text {tor }}}\right)\right) \cong H^{1}\left(\mathfrak{N}_{\breve{\sigma}, \tau}, \underline{\mathcal{H}}^{0}\left(\mathcal{O}_{\left(\mathrm{N}^{\text {tor }}\right)} \hat{\tilde{\mathrm{Z}}}_{\left[\left(\Phi_{\mathcal{H}}, \delta \mathcal{H}, \tau\right)\right]}\right)\right)
$$


Thus, to show that (5.15) is an isomorphism over $M_{\mathcal{H}}^{\text {tor }} \otimes_{\mathbb{Z}} \mathbb{Q}$, it suffices (by comparison of ranks of locally free sheaves) to show that it induces surjections from subquotients of $\iota^{*} f_{*}^{\text {tor }}\left(\overline{\underline{\operatorname{Der}}}_{N^{\text {tor }} / \mathrm{M}_{\mathcal{H}}^{\text {tor }}}\right)$ to these graded pieces over $\mathfrak{X}_{\Phi_{\mathcal{H}}, \delta_{\mathcal{H}}, \tau} \otimes_{\mathbb{Z}} \mathbb{Q}$.

By tensoring the above filtration with $\imath^{*} \bar{\Omega}_{\mathrm{N}^{\text {tor }} / \mathrm{M}_{\mathcal{H}}^{\text {tor }}}$ (and by (3.15)), we obtain a decreasing filtration on $\imath^{*} R^{1} f_{*}^{\text {tor }}\left(\bar{\Omega}_{\mathrm{N}^{\text {tor }} / \mathrm{M}_{\mathcal{H}}^{\text {tor }}}\right)$ with

$$
\operatorname{Gr}^{0}\left(\imath^{*} R^{1} f_{*}^{\text {tor }}\left(\bar{\Omega}_{\mathrm{N}^{\text {tor }} / \mathrm{M}_{\mathcal{H}}^{\text {tor }}}^{1}\right)\right) \cong H^{0}\left(\mathfrak{N}_{\breve{\sigma}, \tau}, \underline{\mathscr{H}}^{1}\left(\imath^{*} \bar{\Omega}_{\mathrm{N}^{\text {tor }} / \mathrm{M}_{\mathcal{H}}^{\text {tor }}}^{1}\right)\right)
$$

and

$$
\operatorname{Gr}^{1}\left(l^{*} R^{1} f_{*}^{\text {tor }}\left(\bar{\Omega}_{\mathrm{N}^{\text {tor }} / \mathrm{M}_{\mathcal{H}}^{\text {tor }}}^{1}\right)\right) H^{1}\left(\mathfrak{N}_{\breve{\sigma}, \tau}, \underline{\mathscr{H}}^{0}\left(\imath^{*} \bar{\Omega}_{\mathrm{N}^{\text {tor }} / \mathrm{M}_{\mathcal{H}}^{\text {tor }}}^{1}\right)\right) .
$$

Since $\underline{\overline{\operatorname{Der}}}_{\mathrm{N}^{\text {tor }} / \mathrm{M}_{\mathcal{H}}^{\text {tor }}} \cong\left(f^{\text {tor }}\right)^{*}\left(\underline{\operatorname{Hom}}_{\mathcal{O}}\left(Q, \underline{\operatorname{Lie}}_{G_{\mathrm{M}_{\mathcal{H}}} / \mathrm{M}_{\mathcal{H}}}\right)\right)$, we have

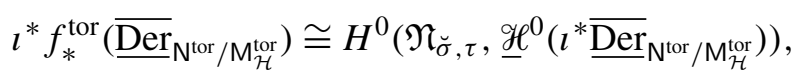

and the morphism

$$
\iota^{*} f_{*}^{\text {tor }}\left(\overline{\operatorname{Der}}_{\mathrm{N}^{\text {tor }} / \mathrm{M}_{\mathcal{H}}^{\text {tor }}}\right) \rightarrow H^{0}\left(\mathfrak{N}_{\breve{\sigma}, \tau}, \underline{\mathscr{H}}^{1}\left(\mathrm{O}_{\left(\mathrm{N}^{\mathrm{tor}}\right)} \hat{\tilde{\mathrm{Z}}}_{\left[\left(\Phi_{\mathcal{H}}, \delta_{\mathcal{H}}, \tau\right)\right]}\right)\right)
$$

induced by (5.15) can be identified with the morphism

$$
H^{0}\left(\mathfrak{N}_{\breve{\sigma}, \tau}, \underline{\mathscr{H}}^{0}\left(\iota^{*} \underline{\overline{\operatorname{Der}}}_{\mathrm{N}^{\text {tor }} / \mathrm{M}_{\mathcal{H}}^{\text {tor }}}\right)\right) \rightarrow H^{0}\left(\mathfrak{N}_{\breve{\sigma}, \tau}, \underline{\mathscr{H}}^{1}\left(\mathrm{O}_{\left(\mathrm{N}^{\text {tor }}\right)} \hat{\tilde{\mathrm{Z}}}_{\left[\left(\Phi_{\mathcal{H}}, \delta \mathcal{H}, \tau\right)\right]}\right)\right)
$$

given by cup product with the image of $\iota^{*}\left(D_{\tilde{\ell}_{j_{Q}}}^{\text {tor }}\right)$ in $\operatorname{Gr}^{0}\left(l^{*} R^{1} f_{*}^{\text {tor }}\left(\bar{\Omega}_{\mathrm{N}^{\text {tor }} / \mathrm{M}_{\mathcal{H}}^{\text {tor }}}^{1}\right)\right) \cong$ $H^{0}\left(\mathfrak{N}_{\breve{\sigma}, \tau}, \underline{\mathcal{H}}^{1}\left(l^{*} \bar{\Omega}_{\mathrm{N}^{\text {tor }} / \mathrm{M}_{\mathcal{H}}^{\text {tor }}}\right)\right)$.

For simplicity, let us define $\widetilde{\mathfrak{X}}_{\Phi_{\mathcal{H}}, \delta_{\mathcal{H}}, \tau}:=\mathfrak{X}_{\Phi_{\mathcal{H}}, \delta_{\mathcal{H}}, \tau} \times_{C_{\Phi_{\mathcal{H}}, \delta_{\mathcal{H}}}}{\widetilde{C_{\Phi}}}_{\check{\Phi}_{\tilde{\mathcal{H}}}, \breve{\delta}_{\tilde{\mathcal{H}}}}$. Then the

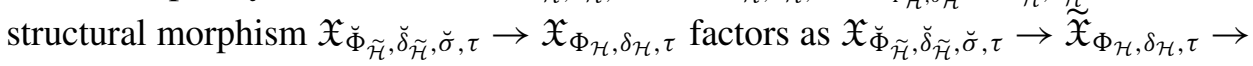
$\mathfrak{X}_{\Phi_{\mathcal{H}}, \delta_{\mathcal{H}}, \tau}$. Over $\mathfrak{X}_{\breve{\Phi}_{\tilde{\mathcal{H}}}, \breve{\delta}_{\tilde{\mathcal{H}}}, \breve{\sigma}, \tau}$, there is an exact sequence

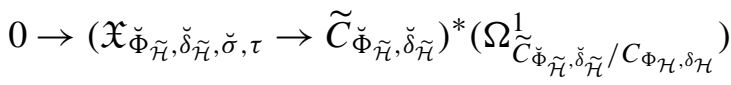

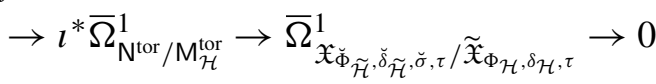

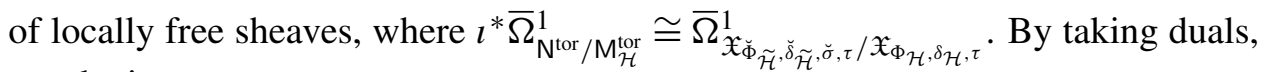
we obtain an exact sequence

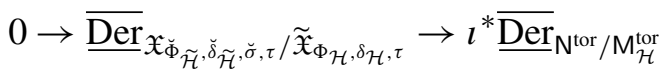

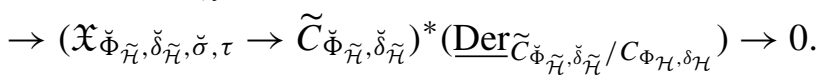

We have similar sequences with $\mathfrak{X}_{\breve{\Phi}_{\tilde{\mathcal{H}}}, \breve{\mathcal{H}}_{\widetilde{\mathcal{H}}}, \breve{\sigma}, \tau}$ replaced with the locally isomorphic

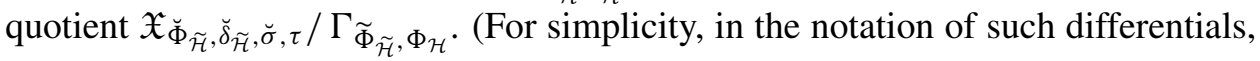
we shall suppress the locally isomorphic quotients by $\left.\Gamma_{\widetilde{\Phi}_{\tilde{\mathcal{H}}}}, \Phi_{\mathcal{H}}.\right)$ 
Since $\Psi_{\breve{\Phi}_{\widetilde{\mathcal{H}}}} \breve{\delta}_{\widetilde{\mathcal{H}}}\left(\breve{\ell}_{j_{Q}, \breve{\tau}}\right)$ is the pullback of an invertible sheaf on $\widetilde{C}_{\breve{\Phi}_{\tilde{\mathcal{H}}}}, \breve{\delta}_{\tilde{\mathcal{H}}}$, the image of $\imath^{*}\left(D_{\tilde{\ell}_{j}}^{\text {tor }}\right)$ in $H^{0}\left(\mathfrak{N}_{\breve{\sigma}, \tau}, \underline{H}^{1}\left(\imath^{*} \bar{\Omega}_{\mathrm{N}^{\text {tor }} / \mathrm{M}_{\mathcal{H}}^{\text {tor }}}\right)\right)$ lies locally over each $\mathfrak{U}_{\breve{\tau}}$ in the image of

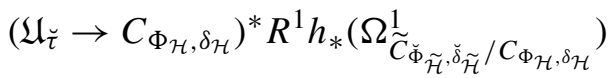

$$
\begin{aligned}
& \stackrel{\sim}{\longrightarrow} \underline{\mathscr{H}}^{1}\left(\left(\mathfrak{U}_{\breve{\tau}} \rightarrow \widetilde{C}_{\breve{\Phi}_{\tilde{\mathcal{H}}}, \breve{\delta}_{\widetilde{\mathcal{H}}}}\right)^{*}\left(\Omega_{\widetilde{C}_{\breve{\Phi}_{\tilde{\mathcal{H}}}, \check{\delta}_{\tilde{\mathcal{H}}}}^{1} / C_{\Phi_{\mathcal{H}}, \delta \mathcal{H}}}\right)\right) \rightarrow \underline{\mathcal{H}}^{1}\left(l^{*} \bar{\Omega}_{\mathrm{N}^{\text {tor }} / \mathrm{M}_{\mathcal{H}}^{\text {tor }}}^{1}\right) .
\end{aligned}
$$

Hence (5.17) factors as

$$
\begin{aligned}
& H^{0}\left(\mathfrak{N}_{\breve{\sigma}, \tau}, \underline{\mathscr{H}}^{0}\left(\imath^{*}{\underline{\underline{\operatorname{Der}}}}_{\mathrm{N}^{\text {tor }} / \mathrm{M}_{\mathcal{H}}^{\text {tor }}}\right)\right)
\end{aligned}
$$

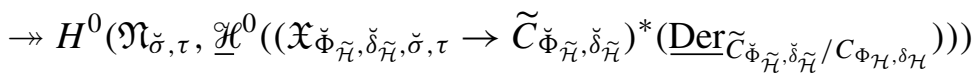

$$
\begin{aligned}
& \stackrel{\sim}{\longrightarrow}\left(\mathfrak{X}_{\Phi_{\mathcal{H}}, \delta_{\mathcal{H}}, \tau} \rightarrow C_{\Phi_{\mathcal{H}}, \delta_{\mathcal{H}}}\right)^{*} R^{0} h_{*}\left(\underline{\operatorname{Der}} \widetilde{C}_{\breve{\Phi}_{\tilde{\mathcal{H}}}, \breve{\delta}_{\tilde{\mathcal{H}}}} / C_{\Phi_{\mathcal{H}}, \delta_{\mathcal{H}}}\right)
\end{aligned}
$$

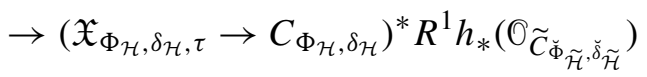

$$
\begin{aligned}
& \stackrel{\sim}{\longrightarrow} H^{0}\left(\mathfrak{N}_{\breve{\sigma}, \tau}, \underline{\mathscr{H}}^{1}\left(\mathcal{O}_{\left(\mathrm{N}^{\mathrm{tor}}\right)} \hat{\tilde{\mathrm{Z}}}_{\left[\left(\Phi_{\mathcal{H}}, \delta_{\mathcal{H}}, \tau\right)\right]}\right)\right) \text {. }
\end{aligned}
$$

Lemma 5.18. The morphism

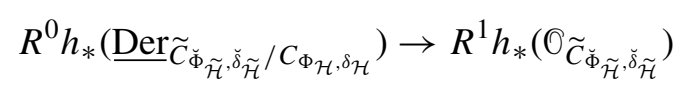

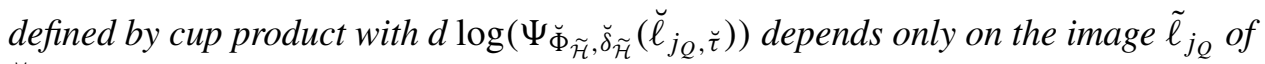
$\breve{\ell}_{j_{Q}, \breve{\tau}}$ in $\mathbf{S}_{\widetilde{\Phi}_{\tilde{\mathcal{H}}}}$ under the second morphism in (3.6) (and hence is independent of the choice of $\left.\breve{\ell}_{j_{Q}, \breve{\tau}}\right)$. Moreover, this morphism is surjective over $\mathfrak{X}_{\Phi_{\mathcal{H}}, \delta_{\mathcal{H}}, \tau} \otimes_{\mathbb{Z}} \mathbb{Q}$.

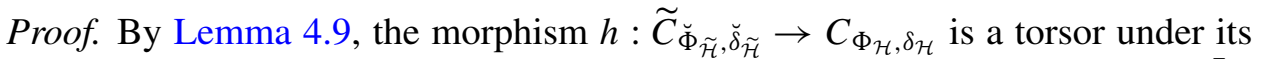
kernel $C$, which is an abelian scheme $\mathbb{Z}_{(\square)}^{\times}$-isogenous to $\underline{\operatorname{Hom}}_{\mathcal{O}}(Q, A)^{\circ} \rightarrow \mathrm{M}_{\mathcal{H}}^{\mathrm{Z}_{\mathcal{H}}}$. The restriction of $\Psi_{\breve{\Phi}_{\tilde{\mathcal{H}}}, \breve{\delta}_{\tilde{\mathcal{H}}}}\left(\breve{\ell}_{j_{Q}, \breve{\tau}}\right)$ to $C$ depends only on the image $\tilde{\ell}_{j_{Q}}$ of $\breve{\ell}_{j_{Q}, \breve{\tau}}$ in $\widetilde{\sigma}_{0}^{\vee}$, and is relatively ample by the same proofs of Corollary 2.12 and Lemma 5.5 (with $G_{\mathrm{M}_{\mathcal{H}}} \rightarrow \mathrm{M}_{\mathcal{H}}$ replaced with $A \rightarrow \mathrm{M}_{\mathcal{H}}^{Z_{\mathcal{H}}}$ ). Hence the lemma follows.

Corollary 5.19. The morphism (5.17) is surjective over $\mathrm{M}_{\mathcal{H}}^{\text {tor }} \otimes_{\mathbb{Z}} \mathbb{Q}$. Its kernel is the

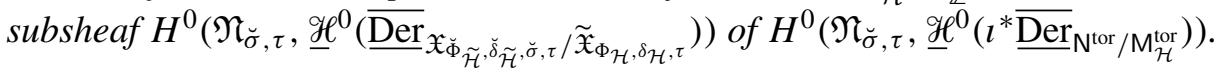

Now consider the induced morphism

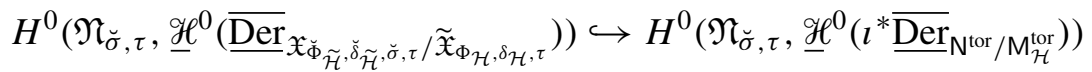

$$
\begin{aligned}
& \stackrel{\sim}{\longrightarrow} R^{0} f_{*}^{\text {tor }}\left(l^{*} \underline{\underline{\operatorname{Der}}}_{\mathrm{N}^{\text {tor }} / \mathrm{M}_{\mathcal{H}}^{\text {tor }}}\right) \rightarrow R^{1} f_{*}^{\text {tor }}\left(\mathcal{O}_{\left(\mathrm{N}^{\text {tor }}\right)} \hat{\tilde{\mathrm{Z}}}_{\left[\left(\Phi_{\mathcal{H}}, \delta_{\mathcal{H}}, \tau\right)\right]}\right)
\end{aligned}
$$

defined by cup product with $\iota^{*}\left(D_{\tilde{\ell}_{j}}^{\text {tor }}\right)$. This composition has image in

$$
H^{1}\left(\mathfrak{N}_{\breve{\sigma}, \tau}, \underline{\mathscr{H}}^{0}\left(\mathcal{O}_{\left(\mathrm{N}^{\text {tor }}\right)} \hat{\tilde{\mathrm{Z}}}_{\left[\left(\Phi_{\mathcal{H}}, \delta \mathcal{H}, \tau\right)\right]}\right)\right)
$$


because its further composition with

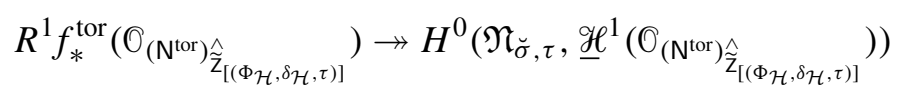

is zero (by Corollary 5.19). Thus the question is whether cup product with $\imath^{*}\left(D_{\tilde{\ell}_{Q}}^{\text {tor }}\right)$ induces a morphism

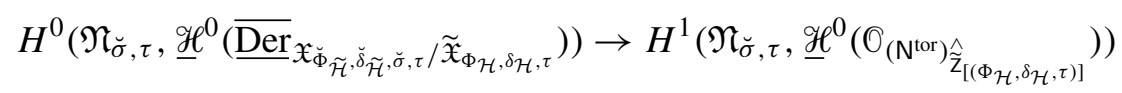

surjective over $\mathfrak{X}_{\Phi_{\mathcal{H}}, \delta_{\mathcal{H}}, \tau} \otimes_{\mathbb{Z}} \mathbb{Q}$.

Lemma 5.21. Suppose $\breve{\tau} \in \Sigma_{\breve{\Phi}_{\widetilde{\mathcal{H}}}, \breve{\sigma}, \tau}$, and $\breve{\ell} \in \breve{\sigma}^{\perp}$. Suppose $\mathfrak{V}$ is an affine open formal subscheme of $\widetilde{\mathfrak{X}}_{\Phi_{\mathcal{H}}, \delta_{\mathcal{H}}, \tau}$ over which the pullback of $\Psi_{\Phi_{\tilde{\mathcal{H}}}, \breve{\delta}_{\tilde{\mathcal{H}}}}(\breve{\ell})$ is a principal ideal of $\mathrm{O}_{\mathfrak{V}}$ generated by some section $x$. Let $\mathfrak{U}:=\mathfrak{U}_{\breve{\tau}} \times \widetilde{\mathfrak{X}}_{\Phi_{\mathcal{H}}, \delta_{\mathcal{H}}, \tau} \mathfrak{V}$ and let $\overline{\widetilde{O}}_{\mathfrak{U}}^{\times, \mathrm{gp}}$ be the pullback of $\overline{\mathrm{O}}_{\mathrm{N}^{\text {tor }}}^{\times, \mathrm{gp}}$ to $\mathfrak{U}$. Let

$$
\overline{\mathrm{O}}_{\mathfrak{V}}^{\times, \mathrm{gp}}:=(\mathfrak{U} \rightarrow \mathfrak{V})_{*}\left(\overline{\mathrm{O}}_{\mathfrak{U}}^{\times, \mathrm{gp}}\right) .
$$

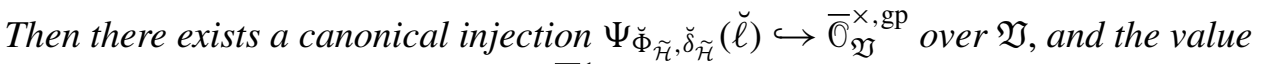
of the section $d \log (x)$ of $(\mathfrak{U} \rightarrow \mathfrak{V})_{*} \bar{\Omega}_{\mathfrak{U} / \mathfrak{X}_{\Phi_{\mathcal{H}}, \delta_{\mathcal{H}}, \tau}}$ determines a canonical section of $\bar{\Omega}_{\mathfrak{U} / \widetilde{\mathfrak{X}}_{\Phi_{\mathcal{H}}, \delta_{\mathcal{H}}, \tau}}^{1}$ (which is independent of the choice of the generator $x$ ).

Proof. If we replace $x$ with $a x$, for some $a \in \mathbb{O}_{\mathfrak{V}}^{\times}$, then $d \log (a x)=d \log (a)+$ $d \log (x)=d \log (x)$ because $d \log (a)=0$ in $(\mathfrak{U} \rightarrow \mathfrak{V})_{*} \bar{\Omega}_{\mathfrak{U} / \mathfrak{X}_{\Phi_{\mathcal{H}}, \delta_{\mathcal{H}}, \tau}}$.

Corollary 5.22. Suppose $\breve{\tau} \in \Sigma_{\breve{\Phi}_{\tilde{\mathcal{H}}}, \breve{\sigma}, \tau}$, and $\breve{\ell} \in \breve{\sigma}^{\perp}$. Then the local generators of $\Psi_{\Phi_{\tilde{\mathcal{H}}}, \breve{\delta}_{\widetilde{\mathcal{H}}}}(\breve{\ell})$ in Lemma 5.21 determine a well-defined section of $\bar{\Omega}_{\mathfrak{U}_{\tilde{\tau}} / \tilde{\mathfrak{X}}_{\Phi_{\mathcal{H}}, \delta_{\mathcal{H}}, \tau}}$, which

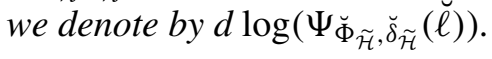

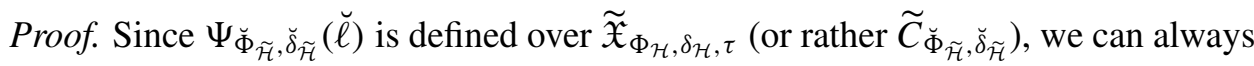
cover $\mathfrak{U}_{\tilde{\tau}}$ by open formal subschemes $\mathfrak{U}$ as in Lemma 5.21.

Lemma 5.23. For any $\breve{\tau}, \breve{\tau}^{\prime} \in \Sigma_{\Phi_{\widetilde{\mathcal{H}}}, \breve{\sigma}, \tau}$ such that $\breve{\tau}$ and $\breve{\tau}^{\prime}$ are adjacent to each

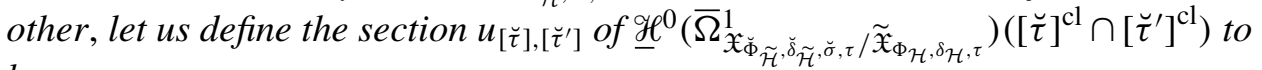
be

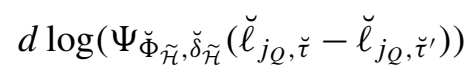

(as in Corollary 5.22). Then this is well defined and determines a section $u$ of $H^{1}\left(\mathfrak{N}_{\breve{\sigma}, \tau}, \underline{\mathcal{H}}^{0}\left(\imath^{*} \bar{\Omega}_{\mathrm{N}^{\text {tor }} / \mathrm{M}_{\mathcal{H}}^{\text {tor }}}^{1}\right)\right)$ that induces by cup product the same morphism as (5.20).

Proof. If $\breve{\tau}$ and $\breve{\tau}^{\prime}$ are adjacent, then $\gamma \breve{\tau}$ and $\gamma^{\prime} \breve{\tau}^{\prime}$ are adjacent for $\gamma, \gamma^{\prime} \in \Gamma_{\widetilde{\Phi}_{\tilde{\mathcal{H}}}, \Phi_{\mathcal{H}}}$ only when $\gamma=\gamma^{\prime}$ (by Condition 1.29; cf. Lemma 3.1), in which case

$$
\breve{\ell}_{j_{Q}, \gamma \breve{\tau}}-\breve{\ell}_{j_{Q}, \breve{\tau}}=\gamma \breve{\ell}_{j_{Q}, \breve{\tau}}-\breve{\ell}_{j_{Q}, \breve{\tau}}=\gamma \breve{\ell}_{j_{Q}, \breve{\tau}^{\prime}}-\breve{\ell}_{j_{Q}, \breve{\tau}^{\prime}}=\breve{\ell}_{j_{Q}, \gamma \breve{\tau}^{\prime}}-\breve{\ell}_{j_{Q}, \breve{\tau}^{\prime}}
$$


(because $\Gamma_{\widetilde{\Phi}_{\tilde{\mathcal{H}}}, \Phi_{\mathcal{H}}}$ acts by the same translation on $\breve{\ell}_{j_{Q}, \breve{\tau}}$ and $\breve{\ell}_{j_{Q}, \breve{\tau}^{\prime}}$ ). This shows that the assignment of $u_{[\breve{\tau}],\left[\breve{\tau}^{\prime}\right]}$ is independent of the choices of the respective representatives $\breve{\tau}$ and $\breve{\tau}^{\prime}$ of $[\breve{\tau}]$ and $\left[\breve{\tau}^{\prime}\right]$, and that $u$ is well defined.

Cup product with $u$ induces the same morphism as (5.20) because the canonical morphism

factors through

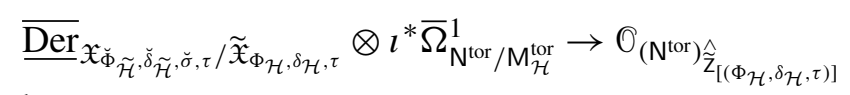

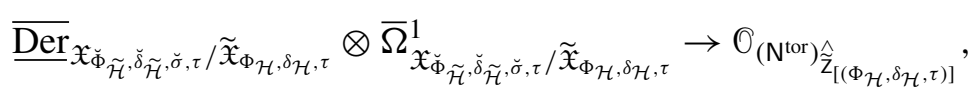

and because cup product with the image of $\iota^{*}\left(D_{\ell_{j_{Q}}}^{\text {tor }}\right)$ in $H^{0}\left(\mathfrak{N}_{\breve{\sigma}, \tau}, \mathscr{H}^{1}\left(l^{*} \bar{\Omega}_{\mathrm{N}^{\text {tor }} / \mathrm{M}_{\mathcal{H}}^{\text {tor }}}\right)\right)$ induces the zero morphism (cf. the paragraph preceding Lemma 5.18).

Consider any sequence $\breve{\tau}_{1}, \breve{\tau}_{2}, \ldots, \breve{\tau}_{k}$ of adjacent cones in $\Sigma_{\breve{\Phi}}, \breve{\mathcal{H}}, \tau$, such that $\breve{\tau}_{k}=\gamma \breve{\tau}_{1}$ for some $\gamma \in \Gamma_{\widetilde{\Phi}_{\tilde{\mathcal{H}}}}, \Phi_{\mathcal{H}}$. The union of the cones in any such sequence form a subset of $\widetilde{\mathfrak{N}}_{\breve{\sigma}, \tau}$ contractible to a path joining a point in $\breve{\tau}$ with its translation by $\gamma$ in $\gamma \breve{\tau}$, whose image in $\mathfrak{N}_{\breve{\sigma}, \tau}$ defines a loop. Suppose we have a class $s$ in $H^{1}\left(\mathfrak{N}_{\breve{\sigma}, \tau}, \underline{\mathscr{H}}^{0}\left(\widehat{O}_{\left(\mathrm{N}^{\text {tor }}\right)} \hat{\tilde{\mathrm{Z}}}_{\left[\left(\Phi_{\mathcal{H}}, \mathcal{\delta}_{\mathcal{H}}, \tau\right)\right]}\right)\right)$ represented by a collection of sections

$$
s_{[\breve{\tau}],\left[\breve{\tau}^{\prime}\right]} \in \underline{\mathscr{H}}^{0}\left(\mathrm{O}_{\left(\mathrm{N}^{\mathrm{tor}}\right)} \hat{\mathrm{z}}_{\left[\left(\Phi_{\mathcal{H}}, \delta \mathcal{H}, \tau\right)\right]}\right)\left([\breve{\tau}]^{\mathrm{cl}} \cap\left[\breve{\tau}^{\prime}\right]^{\mathrm{cl}}\right)
$$

for $[\breve{\tau}],\left[\breve{\tau}^{\prime}\right] \in \Sigma_{\breve{\Phi}_{\widetilde{\mathcal{H}}}, \breve{\sigma}, \tau} / \Gamma_{\widetilde{\Phi}_{\widetilde{\mathcal{H}}}, \Phi_{\mathcal{H}}}$, and suppose we define formally $s_{\breve{\tau}, \breve{\tau}^{\prime}}=s_{[\breve{\tau}],\left[\breve{\tau}^{\prime}\right]}$ for

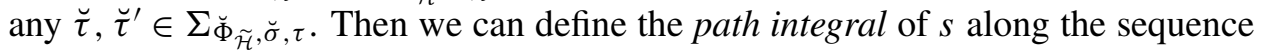
$\breve{\tau}_{1}, \breve{\tau}_{2}, \ldots, \breve{\tau}_{k}$ to be the sum

$$
\sum_{i=1}^{k-1} S_{\breve{\tau}_{i}, \breve{\tau}_{i+1}}
$$

This defines a morphism

$$
H^{1}\left(\mathfrak{N}_{\breve{\sigma}, \tau}, \underline{\mathscr{H}}^{0}\left(\mathcal{O}_{\left(\mathrm{N}^{\text {tor }}\right)} \hat{\tilde{\mathrm{Z}}}_{\left[\left(\Phi_{\mathcal{H}}, \delta \mathcal{H}, \tau\right)\right]}\right)\right) \rightarrow \mathcal{O}_{\mathfrak{X}_{\Phi_{\mathcal{H}}, \delta_{\mathcal{H}}, \tau}}
$$

Note that this is a realization of the cap product

$$
\begin{aligned}
& H_{1}\left(\mathfrak{N}_{\breve{\sigma}, \tau}, \mathbb{Z}\right) \times H^{1}\left(\mathfrak{N}_{\breve{\sigma}, \tau}, \underline{\mathscr{H}}^{0}\left(\mathrm{O}_{\left(\mathrm{N}^{\text {tor }}\right)} \hat{\tilde{\mathrm{Z}}}_{\left[\left(\Phi_{\mathcal{H}}, \delta_{\mathcal{H}}, \tau\right)\right]}\right)\right)
\end{aligned}
$$

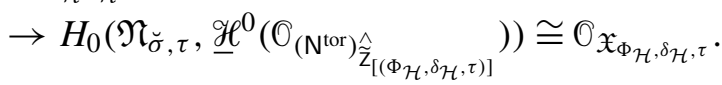

Lemma 5.25. For any $\breve{\ell} \in \mathbf{S}_{\breve{\Phi}_{\tilde{\mathcal{H}}}}$ that is mapped to $\tilde{\ell}_{j_{Q}}$ in $\widetilde{\sigma}_{0}^{\vee}$ under the second

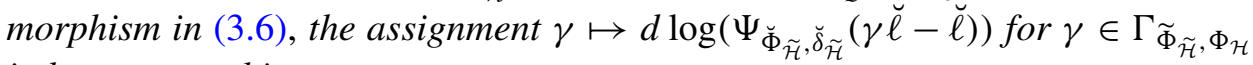
induces a morphism

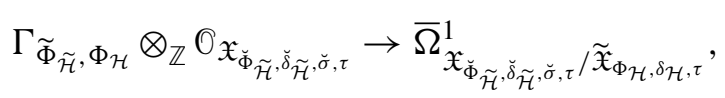

which is an isomorphism over $\mathfrak{X}_{\breve{\Phi}_{\widetilde{\mathcal{H}}}, \breve{\delta}_{\widetilde{\mathcal{H}}}, \breve{\sigma}, \tau} \otimes_{\mathbb{Z}} \mathbb{Q}$. 
Proof. Since $\gamma \breve{\ell}$ and $\breve{\ell}$ have the same image $\tilde{\ell}_{j_{Q}}$ in $\widetilde{\sigma}_{0}^{\vee}$ under the second morphism in (3.6), the difference $\gamma \breve{\ell}-\breve{\ell}$ lands in $\breve{\sigma}^{\perp}$. For any $\breve{\ell}^{\prime} \in \breve{\sigma}^{\perp}$, an elementary matrix calculation (using any splitting of $s_{\breve{X}} \otimes_{\mathbb{Z}} \mathbb{Q}: \breve{X} \otimes_{\mathbb{Z}} \mathbb{Q} \rightarrow \tilde{X} \otimes_{\mathbb{Z}} \mathbb{Q}$ ) shows that $\gamma \breve{\ell^{\prime}}-\breve{\ell^{\prime}}$ lies in $\mathbf{S}_{\Phi_{\mathcal{H}}}=\left(\mathbf{S}_{\Phi_{\mathcal{H}}} \otimes_{\mathbb{Z}} \mathbb{Q}\right) \cap \mathbf{S}_{\Phi_{\tilde{\mathcal{H}}}}$ (identified as the image of the first morphism in (3.6)). Therefore, we have $\left(\gamma_{1} \gamma_{2} \breve{\ell}-\breve{\ell}\right)-\left(\gamma_{1} \breve{\ell}-\breve{\ell}\right)-\left(\gamma_{2} \breve{\ell}-\breve{\ell}\right)=$ $\gamma_{1}\left(\gamma_{2} \breve{\ell}-\breve{\ell}\right)-\left(\gamma_{2} \breve{\ell}-\breve{\ell}\right) \in \mathbf{S}_{\Phi_{\mathcal{H}}}$, which shows that the assignment $\gamma \mapsto \gamma \breve{\ell}-\breve{\ell}$ defines a group homomorphism $\Gamma_{\widetilde{\Phi}_{\tilde{\mathcal{H}}}, \Phi_{\mathcal{H}}} \rightarrow\left(\breve{\sigma}^{\perp} / \mathbf{S}_{\Phi_{\mathcal{H}}}\right)$. By the choice of $j_{Q}$, the element $\tilde{\ell}_{j_{Q}}$ is represented by a positive definite matrix with respect to any choice of basis, and hence the homomorphism $\Gamma_{\widetilde{\Phi}_{\tilde{\mathcal{H}}}, \Phi_{\mathcal{H}}} \rightarrow\left(\breve{\sigma}^{\perp} / \mathbf{S}_{\Phi_{\mathcal{H}}}\right)$ induced by $\gamma \mapsto \gamma \breve{\ell}-\breve{\ell}$ is injective (by another elementary matrix calculation over $\mathbb{Q}$ ). By comparison of dimensions, this shows that the induced injective homomorphism

$$
\Gamma_{\widetilde{\Phi}_{\widetilde{\mathcal{H}}}, \Phi_{\mathcal{H}}} \otimes_{\mathbb{Z}} \mathbb{Q} \rightarrow\left(\breve{\sigma}^{\perp} / \mathbf{S}_{\Phi_{\mathcal{H}}}\right) \otimes_{\mathbb{Z}} \mathbb{Q}
$$

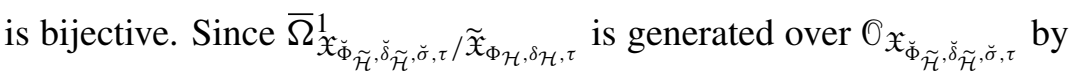

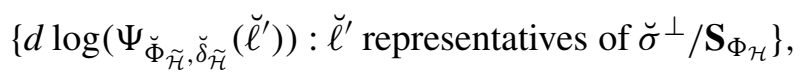

the lemma follows.

Lemma 5.26. Let $\breve{\tau}_{1}, \breve{\tau}_{2}, \ldots, \breve{\tau}_{k}$ be a sequence of adjacent cones in $\Sigma_{\breve{\Phi}_{\widetilde{\mathcal{H}}}, \breve{\sigma}, \tau}$, such

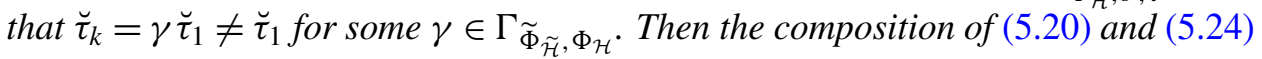
is surjective over $\mathfrak{X}_{\breve{\Phi}_{\tilde{\mathcal{H}}}, \breve{s}_{\tilde{\mathcal{H}}}, \breve{\sigma}, \tau} \otimes_{\mathbb{Z}} \mathbb{Q}$.

Proof. If $\gamma \breve{\tau}_{1} \neq \breve{\tau}_{1}$, then $\breve{\ell}_{j_{Q}, \gamma \breve{\tau}_{1}}=\gamma \breve{\ell}_{j_{Q}, \breve{\tau}_{1}} \neq \breve{\ell}_{j_{Q}, \breve{\tau}_{1}}$ by the proof of Lemma 5.25. By Lemma 5.25 , this implies that $d \log \left(\Psi_{\breve{\Phi}_{\tilde{\mathcal{H}}}, \breve{\delta}_{\widetilde{\mathcal{H}}}}\left(\breve{\ell}_{j_{Q}, \breve{\tau}_{1}}-\breve{\ell}_{j_{Q}, \breve{\tau}_{k}}\right)\right)$ defines a nonzero section of $\bar{\Omega}_{\mathfrak{X}_{\tilde{\Phi} \tilde{\mathcal{H}}, \check{\delta} \tilde{\mathcal{H}}, \check{\sigma}, \tau} / \widetilde{\mathfrak{X}}_{\Phi_{\mathcal{H}}, \delta_{\mathcal{H}}, \tau}}$ over every $\mathfrak{U}_{[\breve{\tau}]} \otimes_{\mathbb{Z}} \mathbb{Q}$. Let $t$ be any section of $H^{0}\left(\mathfrak{N}_{\breve{\sigma}, \tau}, \underline{\mathcal{H}}^{0}\left(l^{*}{\underline{\underline{D e r}^{\prime}}}_{\mathrm{N}^{\text {tor }}} / \mathrm{M}_{\mathcal{H}}^{\text {tor }}\right)\right)$. Cup product with $u$ (see Lemma 5.23) sends $t$ to the class $s$ in $H^{1}\left(\mathfrak{N}_{\breve{\sigma}, \tau}, \mathscr{H}^{0}\left(\mathcal{O}_{\left(\mathrm{N}^{\text {tor }}\right)} \hat{\tilde{\mathrm{Z}}}_{\left[\left(\Phi_{\mathcal{H}}, \delta_{\mathcal{H}}, \tau\right)\right]}\right)\right)$ represented (up to a sign convention) by the collection of sections

$$
s_{[\breve{\tau}],\left[\breve{\tau}^{\prime}\right]} \in \underline{\mathscr{H}}^{0}\left(\mathcal{O}_{\left(\mathrm{N}^{\mathrm{tor}}\right)} \hat{\tilde{\mathrm{z}}}_{\left[\left(\Phi_{\mathcal{H}}, \delta \mathcal{H}, \tau\right)\right]}\right)\left([\breve{\tau}]^{\mathrm{cl}} \cap\left[\breve{\tau}^{\prime}\right]^{\mathrm{cl}}\right)
$$

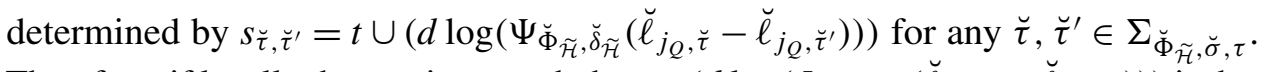

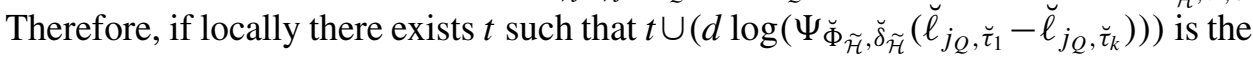
pullback of (local) generators of $\mathcal{O}_{\mathfrak{X}_{\Phi_{\mathcal{H}}, \delta_{\mathcal{H}}, \tau} \otimes_{\mathbb{Z}} \mathbb{Q}}$, which is possible by Lemma 5.25, then the path integral

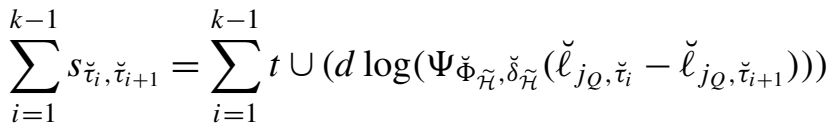

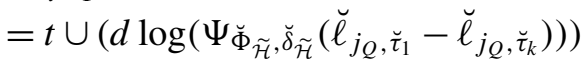

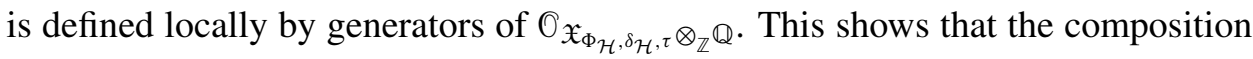
of (5.20) with (5.24) is surjective over $\mathfrak{X}_{\breve{\Phi}_{\widetilde{\mathcal{H}}}, \breve{\delta}_{\widetilde{\mathcal{H}}}, \breve{\sigma}, \tau} \otimes_{\mathbb{Z}} \mathbb{Q}$, as desired. 
Corollary 5.27. The morphism (5.20) is surjective over $\mathfrak{X}_{\breve{\Phi}_{\widetilde{\mathcal{H}}}, \breve{\delta}_{\widetilde{\mathcal{H}}}, \breve{\sigma}, \tau} \otimes_{\mathbb{Z}} \mathbb{Q}$.

Proof. By Lemma 4.21, (4.25), and Lemma 5.25, the morphism (5.20) is surjective

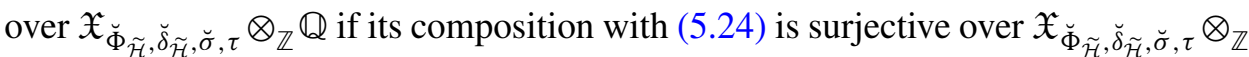
$\mathbb{Q}$ for some collection of sequences $\breve{\tau}_{1}, \breve{\tau}_{2}, \ldots, \breve{\tau}_{k}$ defining loops in $\mathfrak{N}_{\breve{\sigma}, \tau}$ generating $H_{1}\left(\mathfrak{N}_{\breve{\sigma}, \tau}, \mathbb{Z}\right)$. Hence the corollary follows from Lemma 5.26.

Now Proposition 5.14 follows from the combination of Corollaries 5.19 and 5.27. By Lemma 5.2 and Remark 5.4, Proposition 5.14 implies the existence of the canonical isomorphism (5.3). Thus Corollary 4.36 implies:

Corollary 5.28. For any integer $b \geq 0$, we have a canonical isomorphism

$$
R^{b} f_{*}^{\text {tor }}\left(\mathrm{O}_{\mathrm{N}^{\text {tor }}}\right) \cong \Lambda^{b}\left(\underline{\operatorname{Hom}}_{\mathcal{O}}\left(Q^{\vee}, \underline{\operatorname{Lie}}_{G^{\vee}} / \mathrm{M}_{\mathcal{H}}^{\text {tor }}\right)\right)
$$

of locally free sheaves over $\mathrm{M}_{\mathcal{H}}^{\text {tor }}$, compatible with cup products and exterior products, extending the composition of canonical isomorphisms (2.19) over $\mathrm{M}_{\mathcal{H}}$.

This completes the proof of (3b) and (3d) of Theorem 2.15, using respectively (3a) and (3c) of Theorem 2.15. As explained in Section 3E, this also makes (4c) and (5c) of Theorem 2.15 unconditional. The proof of Theorem 2.15 is now complete.

\section{Canonical extensions of principal bundles}

6A. Principal bundles. Consider $\left(G_{\mathrm{M}_{\mathcal{H}}}, \lambda_{\mathrm{M}_{\mathcal{H}}}, i_{\mathrm{M}_{\mathcal{H}}}, \alpha_{\mathcal{H}}\right) \rightarrow \mathrm{M}_{\mathcal{H}}$, the restriction of the degenerating family $\left(G, \lambda, i, \alpha_{\mathcal{H}}\right) \rightarrow \mathrm{M}_{\mathcal{H}}^{\text {tor }}$, which is isomorphic to the tautological tuple over $\mathrm{M}_{\mathcal{H}}$; and consider the relative de Rham cohomology $\underline{H}_{\mathrm{dR}}^{1}\left(G_{\mathrm{M}_{\mathcal{H}}} / \mathrm{M}_{\mathcal{H}}\right)$ and the relative de Rham homology $\underline{H}_{1}^{\mathrm{dR}}\left(G_{\mathrm{M}_{\mathcal{H}}} / \mathrm{M}_{\mathcal{H}}\right):=\underline{\operatorname{Hom}}_{\mathrm{O}_{\mathrm{M}_{\mathcal{H}}}}\left(\underline{H}_{\mathrm{dR}}^{1}\left(G_{\mathrm{M}_{\mathcal{H}}} / \mathrm{M}_{\mathcal{H}}\right), O_{\mathrm{M}_{\mathcal{H}}}\right)$. We have the canonical pairing $\langle\cdot, \cdot\rangle_{\lambda}: \underline{H}_{1}^{\mathrm{dR}}\left(G_{\mathrm{M}_{\mathcal{H}}} / \mathrm{M}_{\mathcal{H}}\right) \times \underline{H}_{1}^{\mathrm{dR}}\left(G_{\mathrm{M}_{\mathcal{H}}} / \mathrm{M}_{\mathcal{H}}\right) \rightarrow$ $\mathrm{O}_{\mathrm{M}_{\mathcal{H}}}(1)$ defined as the composition of $\left(\mathrm{Id} \times \lambda_{\mathrm{M}_{\mathcal{H}}}\right)_{*}$ followed by the perfect pairing $\underline{H}_{1}^{\mathrm{dR}}\left(G_{\mathrm{M}_{\mathcal{H}}} / \mathrm{M}_{\mathcal{H}}\right) \times \underline{H}_{1}^{\mathrm{dR}}\left(G_{\mathrm{M}_{\mathcal{H}}}^{\vee} / \mathrm{M}_{\mathcal{H}}\right) \rightarrow \mathrm{O}_{\mathrm{M}_{\mathcal{H}}}(1)$ defined by the first Chern class of the Poincaré invertible sheaf over $G_{\mathrm{M}_{\mathcal{H}}} \times_{\mathrm{M}_{\mathcal{H}}} G_{\mathrm{M}_{\mathcal{H}}}^{\vee}$. (See for example [Deligne and Pappas 1994, 1.5].) Under the assumption that $\lambda_{\mathrm{M}_{\mathcal{H}}}$ has degree prime to $\square$, we know that $\lambda_{M_{\mathcal{H}}}$ is separable, that $\left(\lambda_{M_{\mathcal{H}}}\right)_{*}$ is an isomorphism, and hence that the pairing $\langle\cdot, \cdot\rangle_{\lambda}$ above is perfect. Let $\langle\cdot, \cdot\rangle_{\lambda}$ also denote the induced pairing on $\underline{H}_{\mathrm{dR}}^{1}\left(G_{\mathrm{M}_{\mathcal{H}}} / \mathrm{M}_{\mathcal{H}}\right) \times \underline{H}_{\mathrm{dR}}^{1}\left(G_{\mathrm{M}_{\mathcal{H}}} / \mathrm{M}_{\mathcal{H}}\right)$ by duality. By [Berthelot et al. 1982, Lemma 2.5.3], we have canonical short exact sequences

$$
0 \rightarrow \underline{\mathrm{Lie}}_{G_{\mathrm{M}_{\mathcal{H}}}^{\vee} / \mathrm{M}_{\mathcal{H}}}^{\vee} \rightarrow \underline{H}_{1}^{\mathrm{dR}}\left(G_{\mathrm{M}_{\mathcal{H}}} / \mathrm{M}_{\mathcal{H}}\right) \rightarrow \underline{\mathrm{Lie}}_{G_{\mathrm{M}_{\mathcal{H}}} / \mathrm{M}_{\mathcal{H}}} \rightarrow 0
$$

and

$$
0 \rightarrow \underline{\mathrm{Lie}}_{G_{\mathrm{M}_{\mathcal{H}}}^{\vee} / \mathrm{M}_{\mathcal{H}}}^{\vee} \rightarrow \underline{H}_{\mathrm{dR}}^{1}\left(G_{\mathrm{M}_{\mathcal{H}}} / \mathrm{M}_{\mathcal{H}}\right) \rightarrow \underline{\mathrm{Lie}}_{G_{\mathrm{M}_{\mathcal{H}}}^{\vee} / \mathrm{M}_{\mathcal{H}}} \rightarrow 0 .
$$

The submodules $\underline{\mathrm{Lie}}_{G_{\mathrm{M}_{\mathcal{H}}}^{\vee} / \mathrm{M}_{\mathcal{H}}}^{\vee}$ and $\underline{\mathrm{Lie}}_{G_{\mathrm{M}_{\mathcal{H}}} / \mathrm{M}_{\mathcal{H}}}^{\vee}$ are maximal totally isotropic with respect to $\langle\cdot, \cdot\rangle_{\lambda}$. 
Consider the $\mathcal{O} \otimes_{\mathbb{Z}} \mathbb{C}$-module

$$
L \otimes_{\mathbb{Z}} \mathbb{C} \rightarrow\left(L \otimes_{\mathbb{Z}} \mathbb{C}\right) / \mathrm{P}_{h},
$$

where $\mathrm{P}_{h}:=\left\{\sqrt{-1} x-h(\sqrt{-1}) x: x \in L \otimes_{\mathbb{Z}} \mathbb{R}\right\} \subset L \otimes_{\mathbb{Z}} \mathbb{C}$.

Now suppose there exists a finite extension $F_{0}^{\prime}$ of $F_{0}$ in $\mathbb{C}$, and a subset $\square^{\prime}$ of $\square$, such that $F_{0}^{\prime}$ is unramified at all primes in $\square^{\prime}$, and such that, by setting $R_{0}:=\mathcal{O}_{F_{0}^{\prime},\left(\square^{\prime}\right)}$, there exists an $\mathcal{O} \otimes_{\mathbb{Z}} R_{0}$-module $L_{0}$ such that $L_{0} \otimes_{R_{0}} \mathbb{C} \cong\left(L \otimes_{\mathbb{Z}} \mathbb{C}\right) / \mathrm{P}_{h}$. Once the choice of $F_{0}^{\prime}$ is fixed, the choice of $L_{0}$ is unique up to isomorphism because $\mathcal{O} \otimes_{\mathbb{Z}} R_{0}$-modules are uniquely determined by their multiranks. (See [Lan 2008, Lemma 1.1.3.4 and Definition 1.1.3.5] for the notion of multiranks.) Let

$$
\langle\cdot, \cdot\rangle_{\text {can. }}:\left(L_{0} \oplus L_{0}^{\vee}(1)\right) \times\left(L_{0} \oplus L_{0}^{\vee}(1)\right) \rightarrow R_{0}(1)
$$

be the alternating pairing defined by $\left\langle\left(x_{1}, f_{1}\right),\left(x_{2}, f_{2}\right)\right\rangle_{\text {can. }}:=f_{2}\left(x_{1}\right)-f_{1}\left(x_{2}\right)$ (cf. [Lan 2008, Lemma 1.1.4.16]).

Definition 6.2. For any $R_{0}$-algebra $R$, set

$$
\begin{aligned}
\mathrm{G}_{0}(R) & :=\left\{\begin{array}{c}
(g, r) \in \mathrm{GL}_{\mathcal{O} \otimes_{\mathbb{Z}} R}\left(\left(L_{0} \oplus L_{0}^{\vee}(1)\right) \otimes_{R_{0}} R\right) \times \mathbf{G}_{\mathrm{m}}(R): \\
\langle g x, g y\rangle=r\langle x, y\rangle, \forall x, y \in\left(L_{0} \oplus L_{0}^{\vee}(1)\right) \otimes_{R_{0}} R
\end{array}\right\}, \\
\mathrm{P}_{0}(R) & :=\left\{(g, r) \in \mathrm{G}_{0}(R): g\left(L_{0}^{\vee}(1) \otimes_{R_{0}} R\right)=L_{0}^{\vee}(1) \otimes_{R_{0}} R\right\}, \\
\mathrm{M}_{0}(R) & :=\mathrm{GL}_{\mathcal{O} \otimes_{\mathbb{Z}} R}\left(L_{0}^{\vee}(1) \otimes_{R_{0}} R\right) \times \mathbf{G}_{\mathrm{m}}(R),
\end{aligned}
$$

where we view $\mathrm{M}_{0}(R)$ canonically as a quotient of $\mathrm{P}_{0}(R)$ by

$$
\mathrm{P}_{0}(R) \rightarrow \mathrm{M}_{0}(R):(g, r) \mapsto\left(\left.g\right|_{L_{0}^{\vee}(1) \otimes_{R_{0}} R}, r\right) .
$$

The assignments are functorial in $R$ and define group functors $\mathrm{G}_{0}, \mathrm{P}_{0}$, and $\mathrm{M}_{0}$ over $R_{0}$.

Lemma 6.3. For any complete local ring $R$ over $R_{0}$ with separably closed residue field, there is an isomorphism

$$
\left(L \otimes_{\mathbb{Z}} R,\langle\cdot, \cdot\rangle\right) \cong\left(L_{0} \oplus L_{0}^{\vee}(1),\langle\cdot, \cdot\rangle_{\text {can. }}\right) \otimes_{R_{0}} R,
$$

and hence an isomorphism $\mathrm{G}(R) \cong \mathrm{G}_{0}(R)$. (Consequently, $\mathrm{P}_{0}(R)$ can be identified with a "parabolic" subgroup of $\mathrm{G}(R)$.)

(In practice, it is not necessary to take $R$ to be complete local. Much smaller rings would suffice for the existence of isomorphisms as in Lemma 6.3.)

In what follows, by abuse of notation, we shall replace $\mathrm{M}_{\mathcal{H}}$ etc. with their base extensions from $\operatorname{Spec}\left(\mathcal{O}_{F_{0},(\square)}\right)$ to $\operatorname{Spec}\left(R_{0}\right)$, and replace $\mathrm{S}_{0}=\operatorname{Spec}\left(\mathcal{O}_{F_{0},(\square)}\right)$ with $\operatorname{Spec}\left(R_{0}\right)$. 
Definition 6.4. The principal $\mathrm{P}_{0}$-bundle over $\mathrm{M}_{\mathcal{H}}$ is the $\mathrm{P}_{0}$-torsor

$$
\begin{aligned}
\mathcal{E}_{\mathrm{P}_{0}}:=\underline{\operatorname{Isom}}_{\mathcal{O} \otimes_{\mathbb{Z}} \mathrm{O}_{\mathrm{M}_{\mathcal{H}}}}\left(\left(\underline{H}_{1}^{\mathrm{dR}}\left(G_{\mathrm{M}_{\mathcal{H}}} / \mathrm{M}_{\mathcal{H}}\right),\langle\cdot, \cdot\rangle_{\lambda}, \mathrm{O}_{\mathrm{M}_{\mathcal{H}}}(1), \underline{\operatorname{Lie}}_{G_{\mathrm{M}_{\mathcal{H}}}^{\vee}}^{\vee} \mathrm{M}_{\mathcal{H}}\right),\right. \\
\left.\left(\left(L_{0} \oplus L_{0}^{\vee}(1)\right) \otimes_{R_{0}} \mathrm{O}_{\mathrm{M}_{\mathcal{H}}},\langle\cdot, \cdot\rangle_{\text {can. }}, \mathrm{O}_{\mathrm{M}_{\mathcal{H}}}(1), L_{0}^{\vee}(1) \otimes_{R_{0}} \mathrm{O}_{\mathrm{M}_{\mathcal{H}}}\right)\right),
\end{aligned}
$$

the sheaf of isomorphisms of $O_{\mathrm{M}_{\mathcal{H}}}$-sheaves of symplectic $\mathcal{O}$-modules with maximal totally isotropic $\mathcal{O} \otimes_{\mathbb{Z}} R_{0}$-submodules. (The group $\mathrm{P}_{0}$ acts as automorphisms on $\left(L \otimes_{\mathbb{Z}} O_{\mathrm{M}_{\mathcal{H}}},\langle\cdot, \cdot\rangle_{\lambda}, \mathrm{O}_{\mathrm{M}_{\mathcal{H}}}(1), L_{0}^{\vee}(1) \otimes_{R_{0}} \mathrm{O}_{\mathrm{M}_{\mathcal{H}}}\right)$ by definition. The third entries in the tuples represent the values of the pairings.)

Definition 6.5. The principal $\mathrm{M}_{0}$-bundle over $\mathrm{M}_{\mathcal{H}}$ is the $\mathrm{M}_{0}$-torsor

$$
\mathcal{E}_{\mathrm{M}_{0}}:=\underline{\operatorname{Isom}}_{\mathcal{O}} \otimes_{\mathbb{Z}} \mathcal{O}_{\mathrm{M}_{\mathcal{H}}}\left(\left(\underline{\operatorname{Lie}}_{G_{\mathrm{M}_{\mathcal{H}}}^{\vee} / \mathrm{M}_{\mathcal{H}}}^{\vee}, \mathcal{O}_{\mathrm{M}_{\mathcal{H}}}(1)\right),\left(L_{0}^{\vee}(1) \otimes_{R_{0}} \mathcal{O}_{\mathrm{M}_{\mathcal{H}}}, \mathcal{O}_{\mathrm{M}_{\mathcal{H}}}(1)\right)\right),
$$

the sheaf of isomorphisms of $\mathcal{O}_{\mathrm{M}_{\mathcal{H}}}$-sheaves of $\mathcal{O} \otimes_{\mathbb{Z}} R_{0}$-modules. (We view the second entries in the pairs as an additional structure, inherited from the corresponding objects for $\mathrm{P}_{0}$. The group $\mathrm{M}_{0}$ acts obviously on $\left(L_{0}^{\vee}(1) \otimes_{R_{0}} \mathrm{O}_{\mathrm{M}_{\mathcal{H}}}, \mathrm{O}_{\mathrm{M}_{\mathcal{H}}}(1)\right)$ as automorphisms, by definition.)

These define étale torsors because, by the theory of infinitesimal deformations (cf. for example [Lan 2008, Chapter 2]) and the theory of Artin's approximations (cf. [Artin 1969, Theorem 1.10 and Corollary 2.5]),

$$
\left(\underline{H}_{1}^{\mathrm{dR}}\left(G_{\mathrm{M}_{\mathcal{H}}} / \mathrm{M}_{\mathcal{H}}\right),\langle\cdot, \cdot\rangle_{\lambda}, \mathrm{O}_{\mathrm{M}_{\mathcal{H}}}(1), \underline{\mathrm{Lie}}_{G_{\mathrm{M}_{\mathcal{H}}}^{\vee} / \mathrm{M}_{\mathcal{H}}}^{\vee}\right)
$$

and

$$
\left(\left(L_{0} \oplus L_{0}^{\vee}(1)\right) \otimes_{R_{0}} \mathrm{O}_{\mathrm{M}_{\mathcal{H}}},\langle\cdot, \cdot\rangle_{\text {can. }}, \mathrm{O}_{\mathrm{M}_{\mathcal{H}}}(1), L_{0}^{\vee}(1) \otimes_{R_{0}} \mathrm{O}_{\mathrm{M}_{\mathcal{H}}}\right)
$$

are étale locally isomorphic.

Definition 6.6. For any $R_{0}$-algebra $E$, we denote by $\operatorname{Rep}_{E}\left(\mathrm{P}_{0}\right)\left(\operatorname{resp} . \operatorname{Rep}_{E}\left(\mathrm{M}_{0}\right)\right)$ the category of $E$-modules with algebraic actions of $\mathrm{P}_{0} \otimes_{R_{0}} E$ (resp. $\mathrm{M}_{0} \otimes_{R_{0}} E$ ).

Definition 6.7. Let $E$ be any $R_{0}$-algebra. For any $W \in \operatorname{Rep}_{E}\left(\mathrm{P}_{0}\right)$, we define

$$
\mathcal{E}_{\mathrm{P}_{0}, E}(W):=\left(\mathcal{E}_{\mathrm{P}_{0}} \otimes_{R_{0}} E\right) \times{ }^{\mathrm{P}_{0} \otimes_{R_{0}} E} W,
$$

called the automorphic sheaf over $\mathrm{M}_{\mathcal{H}} \otimes_{R_{0}} E$ associated with $W$. It is called an automorphic bundle if $W$ is locally free of finite rank over $E$. We define similarly $\mathcal{E}_{\mathrm{M}_{0}, E}(W)$ for $W \in \operatorname{Rep}_{E}\left(\mathrm{M}_{0}\right)$ by replacing $\mathrm{P}_{0}$ with $\mathrm{M}_{0}$ in the above expression.

Lemma 6.8. Let $E$ be any $R_{0}$-algebra. If we view an element $W \in \operatorname{Rep}_{E}\left(\mathrm{M}_{0}\right)$ as an element in $\operatorname{Rep}_{E}\left(\mathrm{P}_{0}\right)$ via the canonical surjection $\mathrm{P}_{0} \rightarrow \mathrm{M}_{0}$, then we have a canonical isomorphism $\mathcal{E}_{\mathrm{P}_{0}, E}(W) \cong \mathcal{E}_{\mathrm{M}_{0}, E}(W)$. 
6B. Canonical extensions. By taking $Q=\mathcal{O}$, so that $\underline{\operatorname{Hom}}_{\mathcal{O}}\left(Q, G_{\mathrm{M}_{\mathcal{H}}}\right)^{\circ} \cong G_{\mathrm{M}_{\mathcal{H}}}$ and so that there exists some $\mathbb{Z}_{(\square)}^{\times}$-isogeny $\kappa^{\text {isog }}: G_{\mathrm{M}_{\mathcal{H}}} \rightarrow \mathrm{N}$ as in Theorem 2.15, the locally free sheaf $\underline{H}_{\mathrm{dR}}^{1}\left(\mathrm{~N} / \mathrm{M}_{\mathcal{H}}\right) \cong \underline{H}_{\mathrm{dR}}^{1}\left(G_{\mathrm{M}_{\mathcal{H}}} / \mathrm{M}_{\mathcal{H}}\right)$ extends to the locally free sheaf $\underline{H}_{\log -\mathrm{dR}}^{1}\left(\mathrm{~N}^{\text {tor }} / \mathrm{M}_{\mathcal{H}}^{\text {tor }}\right)$ over $\mathrm{O}_{\mathrm{M}_{\mathcal{H}}^{\text {tor }}}$. Let

$$
\underline{H}_{1}^{\log -\mathrm{dR}}\left(\mathrm{N}^{\text {tor }} / \mathrm{M}_{\mathcal{H}}^{\text {tor }}\right):=\underline{\operatorname{Hom}}_{\mathrm{M}_{\mathcal{H}}^{\text {tor }}}\left(\underline{H}_{\log -\mathrm{dR}}^{1}\left(\mathrm{~N}^{\text {tor }} / \mathrm{M}_{\mathcal{H}}^{\text {tor }}\right), \mathrm{O}_{\mathrm{M}_{\mathcal{H}}^{\text {tor }}}\right) .
$$

Proposition 6.9. There exists a unique locally free sheaf $\underline{H}_{1}^{\mathrm{dR}}\left(G_{\mathrm{M}_{\mathcal{H}}} / \mathrm{M}_{\mathcal{H}}\right)^{\mathrm{can}}$ over $\mathrm{O}_{\mathrm{M}_{\mathcal{H}}}$ satisfying the following properties:

(1) The sheaf $\underline{H}_{1}^{\mathrm{dR}}\left(G_{\mathrm{M}_{\mathcal{H}}} / \mathrm{M}_{\mathcal{H}}\right)^{\mathrm{can}}$, canonically identified as a subsheaf of the quasicoherent sheaf $\left(\mathrm{M}_{\mathcal{H}} \hookrightarrow \mathrm{M}_{\mathcal{H}}^{\text {tor }}\right)_{*}\left(\underline{H}_{1}^{\mathrm{dR}}\left(G_{\mathrm{M}_{\mathcal{H}}} / \mathrm{M}_{\mathcal{H}}\right)\right)$, is self-dual under the pairing $\left(\mathrm{M}_{\mathcal{H}} \hookrightarrow \mathrm{M}_{\mathcal{H}}^{\text {tor }}\right)_{*}\langle\cdot, \cdot\rangle_{\lambda}$. We shall denote the induced pairing by $\langle\cdot, \cdot\rangle_{\lambda}^{\mathrm{can}}$.

(2) $\underline{H}_{1}^{\mathrm{dR}}\left(G_{\mathrm{M}_{\mathcal{H}}} / \mathrm{M}_{\mathcal{H}}\right)^{\mathrm{can}}$ contains $\underline{\mathrm{Lie}}_{G^{\vee} / \mathrm{M}_{\mathcal{H}}^{\mathrm{tor}}}^{\mathrm{a}}$ as a subsheaf totally isotropic under $\langle\cdot, \cdot\rangle_{\lambda}^{\mathrm{can}}$.

(3) The quotient sheaf $\underline{H}_{1}^{\mathrm{dR}}\left(G_{\mathrm{M}_{\mathcal{H}}} / \mathrm{M}_{\mathcal{H}}\right)^{\mathrm{can}} / \underline{\mathrm{Lie}}_{G^{\mathrm{v}} / \mathrm{M}_{\mathcal{H}}^{\mathrm{tor}}}^{\mathrm{c}}$ can be canonically identified with the subsheaf $\underline{\mathrm{Lie}}_{G / \mathrm{M}_{\mathcal{H}}^{\text {tor }}}$ of $\left(\mathrm{M}_{\mathcal{H}} \hookrightarrow \mathrm{M}_{\mathcal{H}}^{\text {tor }}\right)_{*} \underline{\operatorname{Lie}}_{G_{\mathrm{M}_{\mathcal{H}}} / \mathrm{M}_{\mathcal{H}}}$.

(4) The pairing $\langle\cdot, \cdot\rangle_{\lambda}^{\text {can }}$ induces an isomorphism $\underline{\mathrm{Lie}}_{G / \mathrm{M}_{\mathcal{H}}^{\text {tor }}} \stackrel{\sim}{\longrightarrow} \underline{\mathrm{Lie}}_{G^{\vee}} / \mathrm{M}_{\mathcal{H}}^{\text {tor }}$ which coincides with $d \lambda$.

(5) Let $\underline{H}_{\mathrm{dR}}^{1}\left(G_{\mathrm{M}_{\mathcal{H}}} / \mathrm{M}_{\mathcal{H}}\right)^{\mathrm{can}}:=\underline{\operatorname{Hom}}_{\mathrm{O}_{\mathcal{H}}^{\text {tor }}}\left(\underline{H}_{1}^{\mathrm{dR}}\left(G_{\mathrm{M}_{\mathcal{H}}} / \mathrm{M}_{\mathcal{H}}\right)^{\text {can }}, \mathrm{O}_{\mathrm{M}_{\mathcal{H}}^{\text {tor }}}\right)$. The GaussManin connection

$$
\nabla: \underline{H}_{\mathrm{dR}}^{1}\left(G_{\mathrm{M}_{\mathcal{H}}} / \mathrm{M}_{\mathcal{H}}\right) \rightarrow \underline{H}_{\mathrm{dR}}^{1}\left(G_{\mathrm{M}_{\mathcal{H}}} / \mathrm{M}_{\mathcal{H}}\right) \otimes_{\mathrm{O}_{\mathrm{M}_{\mathcal{H}}}} \Omega_{\mathrm{M}_{\mathcal{H}} / \mathrm{S}_{0}}^{1}
$$

extends to an integrable connection

$$
\nabla: \underline{H}_{\mathrm{dR}}^{1}\left(G_{\mathrm{M}_{\mathcal{H}}} / \mathrm{M}_{\mathcal{H}}\right)^{\mathrm{can}} \rightarrow \underline{H}_{\mathrm{dR}}^{1}\left(G_{\mathrm{M}_{\mathcal{H}}} / \mathrm{M}_{\mathcal{H}}\right)^{\mathrm{can}} \otimes_{\mathcal{O}_{\mathrm{M}_{\mathcal{H}}}} \bar{\Omega}_{\mathrm{M}_{\mathcal{H}}^{\text {tor }} / \mathrm{S}_{0}}^{1}
$$

with log poles along $\mathrm{D}_{\infty, \mathcal{H}}$, called the extended Gauss-Manin connection, such that the composition

$$
\begin{aligned}
& \underline{\mathrm{Lie}_{G / \mathrm{M}_{\mathcal{H}}}^{\vee}} \hookrightarrow \underline{H}_{\mathrm{dR}}^{1}\left(G_{\mathrm{M}_{\mathcal{H}}} / \mathrm{M}_{\mathcal{H}}\right)^{\text {can }} \\
& \quad \stackrel{\nabla}{\rightarrow} \underline{H}_{\mathrm{dR}}^{1}\left(G_{\mathrm{M}_{\mathcal{H}}} / \mathrm{M}_{\mathcal{H}}\right)^{\text {can }} \otimes_{\mathcal{O}_{\mathrm{M}_{\mathcal{H}}}^{\text {tor }}} \bar{\Omega}_{\mathrm{M}_{\mathcal{H}}^{\text {tor }} / \mathrm{S}_{0}}^{1} \rightarrow \underline{\mathrm{Lie}}_{G^{\vee} / \mathrm{M}_{\mathcal{H}}^{\text {tor }}} \otimes_{\mathrm{O}_{\mathrm{M}_{\mathcal{H}}}} \bar{\Omega}_{\mathrm{M}_{\mathcal{H}}^{\text {tor }} / \mathrm{S}_{0}}^{1}
\end{aligned}
$$

induces by duality the extended Kodaira-Spencer morphism

$$
\underline{\mathrm{Lie}}_{G / \mathrm{M}_{\mathcal{H}}^{\mathrm{tor}}}^{\vee} \otimes_{\mathrm{O}_{\mathrm{M}_{\mathcal{H}}^{\text {tor }}}}{\underline{\mathrm{Lie}_{G}^{\vee}}}_{G^{\vee} / \mathrm{M}_{\mathcal{H}}^{\text {tor }}} \rightarrow \bar{\Omega}_{\mathrm{M}_{\mathcal{H}}^{\text {tor }} / \mathrm{S}_{0}}^{1}
$$

in [Lan 2008, Theorem 4.6.3.32], which factors through $\underline{\mathrm{KS}}$ (in Definition 1.40) and induces the extended Kodaira-Spencer isomorphism $\mathrm{KS}_{G / \mathrm{M}_{\mathcal{H}}^{\mathrm{tor}} / \mathrm{S}_{0}}$ in (4) of Theorem 1.41. 
With these characterizing properties, we say that $\left(\underline{H}_{1}^{\mathrm{dR}}\left(G_{\mathrm{M}_{\mathcal{H}}} / \mathrm{M}_{\mathcal{H}}\right)^{\mathrm{can}}, \nabla\right)$ is the canonical extension of $\left(\underline{H}_{1}^{\mathrm{dR}}\left(G_{\mathrm{M}_{\mathcal{H}}} / \mathrm{M}_{\mathcal{H}}\right), \nabla\right)$.

Proof. The uniqueness of $\underline{H}_{1}^{\mathrm{dR}}\left(G_{\mathrm{M}_{\mathcal{H}}} / \mathrm{M}_{\mathcal{H}}\right)^{\text {can }}$ is clear by the first four properties. To show the existence, let us take $\underline{H}_{1}^{\mathrm{dR}}\left(G_{\mathrm{M}_{\mathcal{H}}} / \mathrm{M}_{\mathcal{H}}\right)^{\text {can }}$ to be the sheaf $\underline{H}_{1}^{\text {log-dR }}\left(\mathrm{N}^{\text {tor }} / \mathrm{M}_{\mathcal{H}}^{\text {tor }}\right)$ (for $Q=\mathcal{O}$, as mentioned before this proposition). It is locally free with a Hodge filtration by (3c) of Theorem 2.15. Moreover, by taking some integer $N>0$ prime to $\square$ such that $N$ Diff $^{-1} \subset \mathcal{O}$, we obtain by multiplication by $N$ a morphism $j_{Q}: Q^{\vee} \cong \operatorname{Diff}^{-1} \hookrightarrow Q=\mathcal{O}$ as in Lemma 2.5 such that pullback by $\kappa^{\text {isog }}$ identifies $\langle\cdot, \cdot\rangle_{\lambda_{\mathrm{M}_{\mathcal{H}}, j_{O}}}: \underline{H}_{\mathrm{dR}}^{1}\left(\mathrm{~N} / \mathrm{M}_{\mathcal{H}}\right) \times \underline{H}_{\mathrm{dR}}^{1}\left(\mathrm{~N} / \mathrm{M}_{\mathcal{H}}\right) \rightarrow \mathrm{O}_{\mathrm{M}_{\mathcal{H}}}(1)$ canonically with $\langle\cdot, \cdot\rangle_{\lambda_{\mathrm{M}_{\mathcal{H}}}}: \underline{H}_{\mathrm{dR}}^{1}\left(G_{\mathrm{M}_{\mathcal{H}}} / \mathrm{M}_{\mathcal{H}}\right) \times \underline{H}_{\mathrm{dR}}^{1}\left(G_{\mathrm{M}_{\mathcal{H}}} / \mathrm{M}_{\mathcal{H}}\right) \rightarrow \mathrm{O}_{\mathrm{M}_{\mathcal{H}}}$ (1). Then (1)-(3) follow from (3d) of Theorem 2.15, and (4) follows from Proposition 5.14 (which is used to prove (3b) of Theorem 2.15). It remains to verify (5). By definition, $\underline{H}_{\mathrm{dR}}^{1}\left(G_{\mathrm{M}_{\mathcal{H}}} / \mathrm{M}_{\mathcal{H}}\right)^{\text {can }} \cong \underline{H}_{\log -\mathrm{dR}}^{1}\left(\mathrm{~N}^{\text {tor }} / \mathrm{M}_{\mathcal{H}}^{\text {tor }}\right)$. The existence of $\nabla$ in (6.10) follows from (3e) of Theorem 2.15. By Remark 4.42, the pullback of (6.11) to $M_{\mathcal{H}}$ is induced by the usual Kodaira-Spencer class. Since the extended Kodaira-Spencer morphism in [Lan 2008, Theorem 4.6.3.32] is defined exactly as a morphism induced by the usual Kodaira-Spencer morphism (by normality of $\mathrm{M}_{\mathcal{H}}^{\text {tor }}$ and local freeness of the sheaves involved), it is induced by duality by (6.11), as desired.

Remark 6.12. The notion of canonical extensions is closely related to the notion of regular singularities of algebraic differential equations. (See [Deligne 1970] and [Katz 1971] for the notion of regular singularities. See [Mumford 1977; Faltings and Chai 1990, Chapter VI; Harris 1989; 1990; Milne 1990] for the notion of canonical extensions over $\mathbb{C}$, and see [Mokrane and Tilouine 2002] for an earlier treatment of canonical extensions in mixed characteristics. See in particular [Harris 1989, Theorem 4.2] for the explanation of why and how the two notions are related.)

Then the principal bundle $\mathcal{E}_{\mathrm{P}_{0}}$ extends canonically to a principal bundle $\mathcal{E}_{\mathrm{P}_{0}}^{\text {can }}$ over $\mathrm{M}_{\mathcal{H}}^{\text {tor }}$ by setting

$$
\begin{aligned}
& \mathcal{E}_{\mathrm{P}_{0}}^{\text {can }}:=\underline{\operatorname{Isom}}_{\mathcal{O}} \otimes_{\mathbb{Z}^{\mathcal{O}} \mathrm{M}_{\mathcal{H}}^{\text {tor }}}\left(\left(\underline{H}_{1}^{\mathrm{dR}}\left(G_{\mathrm{M}_{\mathcal{H}}} / \mathrm{M}_{\mathcal{H}}\right)^{\mathrm{can}},\langle\cdot, \cdot\rangle_{\lambda}^{\text {can }}, \mathcal{O}_{\mathrm{M}_{\mathcal{H}}^{\text {tor }}}(1), \underline{\mathrm{Lie}}_{G^{\vee}}^{\vee} / \mathrm{M}_{\mathcal{H}}^{\text {tor }}\right),\right. \\
& \left.\left(\left(L_{0} \oplus L_{0}^{\vee}(1)\right) \otimes_{R_{0}} O_{\mathrm{M}_{\mathcal{H}}^{\text {tor }}}\langle\cdot, \cdot\rangle_{\text {can. }}, O_{\mathrm{M}_{\mathcal{H}}^{\text {tor }}}(1), L_{0}^{\vee}(1) \otimes_{R_{0}} \mathrm{O}_{\mathrm{M}_{\mathcal{H}}^{\text {tor }}}\right)\right),
\end{aligned}
$$

and the principal bundle $\mathcal{E}_{\mathrm{M}_{0}}$ extends canonically to a principal bundle $\mathcal{E}_{\mathrm{M}_{0}}^{\text {can }}$ over $\mathrm{M}_{\mathcal{H}}^{\text {tor }}$ by setting

$$
\mathcal{E}_{\mathrm{M}_{0}}^{\text {can }}:=\underline{\operatorname{Isom}}_{\mathcal{O} \otimes_{\mathbb{Z}^{O}} \widehat{M}_{\mathcal{H}}^{\text {tor }}}\left(\left(\underline{\mathrm{Lie}}_{G^{\vee}}^{\vee} \mathrm{M}_{\mathcal{H}}^{\text {tor }}, \mathcal{O}_{\mathrm{M}_{\mathcal{H}}^{\text {tor }}}(1)\right),\left(L_{0}^{\vee}(1) \otimes_{R_{0}} \mathcal{O}_{\mathrm{M}_{\mathcal{H}}^{\text {tor }}}, \mathcal{O}_{\mathrm{M}_{\mathcal{H}}^{\text {tor }}}(1)\right)\right) .
$$

Definition 6.13. Let $E$ be any $R_{0}$-algebra. For any $W \in \operatorname{Rep}_{E}\left(\mathrm{P}_{0}\right)$, we define

$$
\mathcal{E}_{\mathrm{P}_{0}, E}^{\mathrm{can}}(W):=\left(\mathcal{E}_{\mathrm{P}_{0}}^{\mathrm{can}} \otimes_{R_{0}} E\right) \times{ }^{\mathrm{P}_{0} \otimes_{R_{0}} E} W,
$$


called the canonical extension of $\mathcal{E}_{\mathrm{P}_{0}, E}(W)$, and define

$$
\mathcal{E}_{\mathrm{P}_{0}, E}^{\mathrm{sub}}(W):=\mathcal{E}_{\mathrm{P}_{0}, E}^{\mathrm{can}}(W) \otimes_{\mathcal{O}_{\mathrm{M}_{\mathcal{H}}^{\text {tor }}}} \Phi_{\mathrm{D}_{\infty, \mathcal{H}}},
$$

called the subcanonical extension of $\mathcal{E}_{\mathrm{P}_{0}, E}(W)$, where $\mathscr{I}_{\mathrm{D}_{\infty, \mathcal{H}}}$ is the $\mathcal{O}_{\mathrm{M}_{\mathcal{H}}}$ tor -ideal defining the relative Cartier divisor $\mathrm{D}_{\infty, \mathcal{H}}$ (with its reduced structure) in (3) of Theorem 1.41. We define similarly $\mathcal{E}_{\mathrm{M}_{0}, E}^{\mathrm{can}}(W)$ and $\mathcal{E}_{\mathrm{M}_{0}, E}^{\mathrm{sub}}(W)$ with $\mathrm{P}_{0}$ (and its principal bundle) replaced accordingly with $\mathrm{M}_{0}$ (and its principal bundle).

Lemma 6.14. Let $E$ be any $R_{0}$-algebra. If we view an element in $W \in \operatorname{Rep}_{E}\left(\mathrm{M}_{0}\right)$ as an element in $\operatorname{Rep}_{E}\left(\mathrm{P}_{0}\right)$ in the canonical way, then we have canonical isomorphisms $\mathcal{E}_{\mathrm{P}_{0}, E}^{\text {can }}(W) \cong \mathcal{E}_{\mathrm{M}_{0}, E}^{\text {can }}(W)$ and $\mathcal{E}_{\mathrm{P}_{0}, E}^{\text {sub }}(W) \cong \mathcal{E}_{\mathrm{M}_{0}, E}^{\text {sub }}(W)$.

6C. Fourier-Jacobi expansions. Let us fix a representative $\left(\mathrm{Z}_{n}, \Phi_{n}, \delta_{n}\right)$ of a cusp label $\left[\left(\mathrm{Z}_{n}, \Phi_{n}, \delta_{n}\right)\right]$ for $\mathrm{M}_{\mathcal{H}}$ (as in Section 1C). As usual, we shall omit $\mathrm{Z}_{\mathcal{H}}$ from the notation.

Definition 6.15. The principal $\mathrm{M}_{0}$-bundle over $C_{\Phi_{\mathcal{H}}, \delta_{\mathcal{H}}}$ is the $\mathrm{M}_{0}$-torsor

$$
\begin{aligned}
\mathcal{E}_{\mathrm{M}_{0}}^{\Phi_{\mathcal{H}}, \delta_{\mathcal{H}}}:= & \underline{\operatorname{Isom}}_{\mathcal{O} \otimes_{\mathbb{Z}}{ }^{0} C_{\Phi_{\mathcal{H}}, \delta_{\mathcal{H}}}}\left(\left(\underline{\mathrm{Lie}}_{G^{\vee, \natural} / C_{\Phi_{\mathcal{H}}, \delta_{\mathcal{H}}}^{\vee}}^{\vee}, \mathcal{O}_{C_{\Phi_{\mathcal{H}}, \delta_{\mathcal{H}}}}(1)\right),\right. \\
& \left(L_{0}^{\vee}(1) \otimes_{R_{0}} \mathcal{O}_{\left.\left.C_{\Phi_{\mathcal{H}}, \delta_{\mathcal{H}}}, O_{C_{\Phi_{\mathcal{H}}, \delta_{\mathcal{H}}}}(1)\right)\right),}\right.
\end{aligned}
$$

with conventions as in Definition 6.5.

Then we define $\mathcal{E}_{\mathrm{M}_{0}, E}^{\Phi_{\mathcal{H}}, \delta_{\mathcal{H}}}(W)$ for any $R_{0}$-algebra $E$ and any $W \in \operatorname{Rep}_{E}\left(\mathrm{M}_{0}\right)$ as in Definition 6.7.

Lemma 6.16. Let $E$ be any $R_{0}$-algebra. For any $W \in \operatorname{Rep}_{E}\left(\mathrm{M}_{0}\right)$, there is a canonical isomorphism

$$
\left(\mathfrak{X}_{\Phi_{\mathcal{H}}, \delta_{\mathcal{H}}, \sigma} \rightarrow \mathrm{M}_{\mathcal{H}}^{\text {tor }}\right)^{*} \mathcal{E}_{\mathrm{M}_{0}}^{\mathrm{can}}(W) \cong\left(\mathfrak{X}_{\Phi_{\mathcal{H}}, \delta_{\mathcal{H}}, \sigma} \rightarrow C_{\Phi_{\mathcal{H}}, \delta_{\mathcal{H}}}\right)^{*} \mathcal{E}_{\mathrm{M}_{0}}^{\Phi_{\mathcal{H}}, \delta_{\mathcal{H}}}(W) .
$$

Proof. This is because of the canonical isomorphism

$$
\left(\mathfrak{X}_{\Phi_{\mathcal{H}}, \delta_{\mathcal{H}}, \sigma} \rightarrow \mathrm{M}_{\mathcal{H}}^{\text {tor }}\right)^{*} \underline{\operatorname{Lie}}_{G^{\vee} / \mathrm{M}_{\mathcal{H}}^{\text {tor }}}^{\vee} \cong\left(\mathfrak{X}_{\Phi_{\mathcal{H}}, \delta_{\mathcal{H}}, \sigma} \rightarrow C_{\Phi_{\mathcal{H}}, \delta_{\mathcal{H}}}\right)^{*} \underline{\operatorname{Lie}}_{G^{\vee, \sharp} / C_{\Phi_{\mathcal{H}}, \delta_{\mathcal{H}}}^{\vee}}^{\vee}
$$

By the construction of $\mathfrak{X}_{\Phi_{\mathcal{H}}, \delta_{\mathcal{H}}, \sigma} \rightarrow C_{\Phi_{\mathcal{H}}, \delta_{\mathcal{H}}}$ as a formal completion, we have a natural morphism

$$
\left(\mathfrak{X}_{\Phi_{\mathcal{H}}, \delta_{\mathcal{H}}, \sigma} \rightarrow C_{\Phi_{\mathcal{H}}, \delta_{\mathcal{H}}}\right)_{*} \mathcal{O}_{\mathfrak{X}_{\Phi_{\mathcal{H}}, \delta_{\mathcal{H}}, \sigma}} \rightarrow \prod_{\ell \in \mathbf{S}_{\Phi_{\mathcal{H}}}} \Psi_{\Phi_{\mathcal{H}}, \delta_{\mathcal{H}}}(\ell)
$$

of $\mathbb{O}_{C_{\Phi_{\mathcal{H}}, \delta_{\mathcal{H}}} \text {-modules. By Lemma 6.16, we have the composition of canonical }}$ morphisms 


$$
\begin{aligned}
& \Gamma\left(\mathrm{M}_{\mathcal{H}}^{\text {tor }}, \mathcal{E}_{\mathrm{M}_{0}}^{\mathrm{can}}(W)\right) \rightarrow \Gamma\left(\mathfrak{X}_{\Phi_{\mathcal{H}}, \delta_{\mathcal{H}}, \sigma},\left(\mathfrak{X}_{\Phi_{\mathcal{H}}, \delta_{\mathcal{H}}, \sigma} \rightarrow \mathrm{M}_{\mathcal{H}}^{\text {tor }}\right)^{*} \mathcal{E}_{\mathrm{M}_{0}}^{\mathrm{can}}(W)\right) \\
& \rightarrow \Gamma\left(\mathfrak{X}_{\Phi_{\mathcal{H}}, \delta_{\mathcal{H}}, \sigma},\left(\mathfrak{X}_{\Phi_{\mathcal{H}}, \delta_{\mathcal{H}}, \sigma} \rightarrow C_{\Phi_{\mathcal{H}}, \delta_{\mathcal{H}}}\right)^{*} \mathcal{E}_{\mathrm{M}_{0}}^{\Phi_{\mathcal{H}}, \delta_{\mathcal{H}}}(W)\right)
\end{aligned}
$$

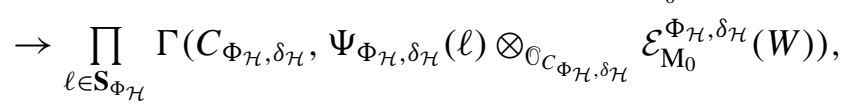

which we call the morphism of algebraic Fourier-Jacobi expansions.

Definition 6.17. The $\ell$-th algebraic Fourier-Jacobi morphism

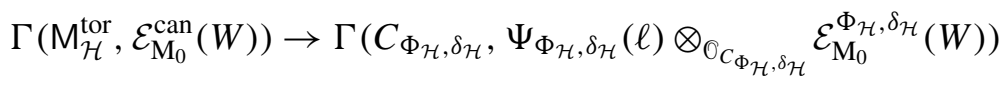

is the $\ell$-th factor of the morphism of algebraic Fourier-Jacobi expansions.

Remark 6.18. If $\mathrm{Gr}_{-1}^{\mathrm{Z}}=\{0\}$, then the abelian scheme $C_{\Phi_{\mathcal{H}}, \delta_{\mathcal{H}}} \rightarrow \mathrm{M}_{n}^{Z_{n}}$ is trivial (i.e., the structural morphism is an isomorphism), and $\mathrm{M}_{n}^{Z_{n}}$ is finite over $\mathrm{S}_{0}=\operatorname{Spec}\left(R_{0}\right)$.

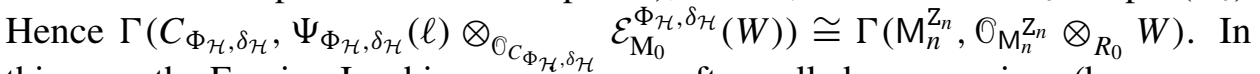
this case, the Fourier-Jacobi expansions are often called $q$-expansions (because no genuine "Jacobi theta functions" are involved).

\section{Acknowledgements}

I would like to thank Richard Taylor and Christopher Skinner for their encouragements, especially in the beginning stage of this work. I would like to thank Tetsushi Ito and Martin Olsson for (independently) bringing to my attention Kazuya Kato's idea of log Picard groups. I would also like to thank Junecue Suh for many helpful discussions.

\section{References}

[Alexeev 2002] V. Alexeev, "Complete moduli in the presence of semiabelian group action", Ann. of Math. (2) 155:3 (2002), 611-708. MR 2003g:14059 Zbl 1052.14017

[Alexeev and Nakamura 1999] V. Alexeev and I. Nakamura, "On Mumford's construction of degenerating Abelian varieties”, Tohoku Math. J. (2) 51:3 (1999), 399-420. MR 2001g:14013 Zbl 0989.14003

[Artin 1969] M. Artin, "Algebraization of formal moduli, I", pp. 21-71 in Global analysis: Papers in honor of K. Kodaira, edited by D. Spencer and S. Iyanaga, Univ. Tokyo, 1969. MR 41 \#5369 Zbl 0205.50402

[Ash et al. 1975] A. Ash, D. Mumford, M. Rapoport, and Y. Tai, Smooth compactification of locally symmetric varieties, Lie groups: History, Frontiers and Applications 4, Math. Sci., Brookline, MA, 1975. MR 56 \#15642 Zbl 0334.14007

[Berthelot et al. 1982] P. Berthelot, L. Breen, and W. Messing, Théorie de Dieudonné cristalline, II, Lecture Notes in Mathematics 930, Springer, Berlin, 1982. MR 667344 (85k:14023) Zbl 0516.14015

[Borel 1969] A. Borel, Introduction aux groupes arithmétiques, Publ. Inst. Math. Univ. Strasbourg 1341, Hermann, Paris, 1969. MR 39 \#5577 Zbl 0186.33202

[Bosch et al. 1990] S. Bosch, W. Lütkebohmert, and M. Raynaud, Néron models, Ergebnisse der Mathematik (3) 21, Springer, Berlin, 1990. MR 1045822 (91i:14034) Zbl 0705.14001 
[Chai 1985] C.-L. Chai, Compactification of Siegel moduli schemes, London Mathematical Society Lecture Note Series 107, Cambridge University Press, 1985. MR 88b:32074 Zbl 0578.14009

[Deligne 1970] P. Deligne, Équations différentielles à points singuliers réguliers, Lecture Notes in Mathematics 163, Springer, Berlin, 1970. MR 54 \#5232 Zbl 0244.14004

[Deligne and Pappas 1994] P. Deligne and G. Pappas, "Singularités des espaces de modules de Hilbert, en les caractéristiques divisant le discriminant", Compositio Math. 90:1 (1994), 59-79. MR 95a:11041 Zbl 0826.14027

[EGA I 1960] A. Grothendieck, "Eléments de géométrie algébrique, I: Le langage des schémas", Inst. Hautes Études Sci. Publ. Math. 4 (1960), 5-228. MR 29 \#1207 Zbl 0118.36206

[EGA III 1 1961] A. Grothendieck, "Eléments de géométrie algébrique, III: Étude cohomologique des faisceaux cohérents, I", Inst. Hautes Études Sci. Publ. Math. 11 (1961), 5-167. MR 0163910 Zbl 0118.36206

[EGA IV IV $_{3}$ 1966] A. Grothendieck, "Eléments de géométrie algébrique, IV: Étude locale des schémas et des morphismes de schémas, III", Inst. Hautes Études Sci. Publ. Math. 28 (1966), 5-255. MR 0217086 Zbl 0144.19904

[EGA IV 4 1967] A. Grothendieck, "Eléments de géométrie algébrique, IV:Étude locale des schémas et des morphismes de schémas, IV”, Inst. Hautes Études Sci. Publ. Math. 32 (1967), 5-361. MR 0238860 Zbl 153.22301

[Faltings 1985] G. Faltings, "Arithmetische Kompaktifizierung des Modulraums der Abelschen Varietäten”, pp. 321-383 in Arbeitstagung Bonn 1984 (Bonn, 1984), edited by F. Hirzebruch et al., Lecture Notes in Mathematics 1111, Springer, Berlin, 1985. MR 87c:14050 Zbl 0597.14036

[Faltings and Chai 1990] G. Faltings and C.-L. Chai, Degeneration of Abelian varieties, Ergebnisse der Mathematik (3) 22, Springer, Berlin, 1990. MR 92d:14036 Zbl 0744.14031

[Fulton and Harris 1991] W. Fulton and J. Harris, Representation theory: a first course, Graduate Texts in Mathematics 129, Springer, New York, 1991. MR 93a:20069 Zbl 0744.22001

[Godement 1958] R. Godement, Topologie algébrique et théorie des faisceaux, Actualités Sci. Ind. Publ. Math. Univ. Strasbourg 1252, Hermann, Paris, 1958. MR 0102797 (21 \#1583) Zbl 0080.16201

[Goodman and Wallach 2009] R. Goodman and N. R. Wallach, Symmetry, representations, and invariants, Graduate Texts in Mathematics 255, Springer, Dordrecht, 2009. MR 2011a:20119 Zbl 1173.22001

[Grothendieck 1971] A. Grothendieck, Eléments de géométrie algébrique I: Le langage des schémas, Grundlehren Math. Wiss. 166, Springer, Berlin, 1971.

[Hakim 1972] M. Hakim, Topos annelés et schémas relatifs, Ergebnisse der Mathematik 64, Springer, Berlin, 1972. MR 51 \#500 Zbl 0246.14004

[Harris 1989] M. Harris, "Functorial properties of toroidal compactifications of locally symmetric varieties", Proc. London Math. Soc. (3) 59:1 (1989), 1-22. MR 90h:11048 Zbl 0711.14011

[Harris 1990] M. Harris, "Automorphic forms and the cohomology of vector bundles on Shimura varieties", pp. 41-91 in Automorphic forms, Shimura varieties, and L-functions (Ann Arbor, MI, 1988), vol. 2, edited by L. Clozel and J. Milne, Perspect. Math. 11, Academic, Boston, MA, 1990. MR 91g:11063 Zbl 0716.14011

[Harris and Zucker 1994] M. Harris and S. Zucker, "Boundary cohomology of Shimura varieties, I: Coherent cohomology on toroidal compactifications", Ann. Sci. École Norm. Sup. (4) 27:3 (1994), 249-344. MR 95a:14024

[Harris and Zucker 2001] M. Harris and S. Zucker, Boundary cohomology of Shimura varieties, III: Coherent cohomology on higher-rank boundary strata and applications to Hodge theory, Mém. Soc. Math. Fr. (N.S.) 85, 2001. MR 1850830 (2002f:14033) Zbl 020.11042 
[Hironaka 1964a] H. Hironaka, "Resolution of singularities of an algebraic variety over a field of characteristic zero, I”, Ann. of Math.(2) 79 (1964), 109-203. MR 33 \#7333 Zbl 0122.38603

[Hironaka 1964b] H. Hironaka, "Resolution of singularities of an algebraic variety over a field of characteristic zero, II”, Ann. of Math. (2) 79 (1964), 205-326. MR 33 \#7333 Zbl 0122.38603

[Illusie 1994] L. Illusie, "Logarithmic spaces (according to K. Kato)", pp. 183-203 in Barsotti symposium in algebraic geometry (Abano Terme, 1991), edited by V. Cristante and W. Messing, Perspect. Math. 15, Academic, San Diego, CA, 1994. MR 95j:14023

[Kato 1989] K. Kato, "Logarithmic structures of Fontaine-Illusie", pp. 191-224 in Algebraic analysis, geometry, and number theory (Baltimore, MD, 1988), edited by J. Igusa, Johns Hopkins, Baltimore, MD, 1989. MR 99b:14020 Zbl 0776.14004

[Katz 1971] N. M. Katz, "The regularity theorem in algebraic geometry”, pp. 437-443 in Actes du congrès international des mathématiciens (Nice, 1970), vol. 1, Gauthier-Villars, Paris, 1971. MR 57 \#12512 Zbl 0235.14006

[Katz 1972] N. M. Katz, "Algebraic solutions of differential equations ( $p$-curvature and the Hodge filtration)", Invent. Math. 18 (1972), 1-118. MR 49 \#2728 Zbl 0278.14004

[Kempf et al. 1973] G. Kempf, F. Knudsen, D. Mumford, and B. Saint-Donat, Toroidal embeddings, I, Lecture Notes in Mathematics 339, Springer, Berlin, 1973. MR 49 \#299 Zbl 0271.14017

[Kottwitz 1992] R. E. Kottwitz, "Points on some Shimura varieties over finite fields", J. Amer. Math. Soc. 5:2 (1992), 373-444. MR 93a:11053 Zbl 0796.14014

[Lan 2008] K.-W. Lan, Arithmetic compactifications of PEL-type Shimura varieties, Ph.D. thesis, Harvard University, 2008, available at http://goo.gl/SygO7. MR 2711676

[Matsumura 1980] H. Matsumura, Commutative algebra, 2nd ed., Mathematics Lecture Note Series 56, Benjamin, Reading, MA, 1980. MR 82i:13003 Zbl 0441.13001

[Milne 1990] J. S. Milne, "Canonical models of (mixed) Shimura varieties and automorphic vector bundles", pp. 283-414 in Automorphic forms, Shimura varieties, and L-functions (Ann Arbor, MI, 1988), vol. 1, Perspect. Math. 10, Academic, Boston, MA, 1990. MR 91a:11027 Zbl 0704.14016

[Mokrane and Tilouine 2002] A. Mokrane and J. Tilouine, "Cohomology of Siegel varieties with p-adic integral coefficients and applications", pp. 1-95 in Cohomology of Siegel varieties, Astérisque 280, 2002. MR 2004c:11081 Zbl 1078.11037

[Mumford 1970] D. Mumford, Abelian varieties, Tata Inst. Fund. Res. Studies in Math. 5, Oxford, 1970. MR 44 \#219 Zbl 0223.14022

[Mumford 1972] D. Mumford, "An analytic construction of degenerating Abelian varieties over complete rings”, Compositio Math. 24 (1972), 239-272. MR 50 \#4593 Zbl 0241.14020

[Mumford 1977] D. Mumford, "Hirzebruch's proportionality theorem in the non-compact case", Invent. Math. 42 (1977), 239-272. MR 81a:32026 Zbl 0365.14012

[Olsson 2004] M. C. Olsson, "Semistable degenerations and period spaces for polarized $K 3$ surfaces", Duke Math. J. 125:1 (2004), 121-203. MR 2005j:14056 Zbl 1073.14054

[Olsson 2008] M. C. Olsson, Compactifying moduli spaces for abelian varieties, Lecture Notes in Mathematics 1958, Springer, Berlin, 2008. MR 2009h:14072 Zbl 1165.14004

[Pink 1990] R. Pink, Arithmetical compactification of mixed Shimura varieties, Bonner Mathematische Schriften 209, Universität Bonn, 1990. MR 92h:11054 Zbl 0748.14007

[Raynaud 1970] M. Raynaud, Faisceaux amples sur les schémas en groupes et les espaces homogènes, Lecture Notes in Mathematics 119, Springer, Berlin, 1970. MR 41 \#5381 Zbl 0195.22701

[Rozensztajn 2006] S. Rozensztajn, "Compactification de schémas Abéliens dégénérant au-dessus d'un diviseur régulier”, Doc. Math. 11 (2006), 57-71. MR 2007b:14096 Zbl 1116.14040 
[SGA 1 1971] A. Grothendieck (editor), Séminaire de Géométrie Algébrique du Bois Marie 19601961: Revêtements étales et groupe fondamental (SGA 1), Lecture Notes in Mathematics 224, Springer, Berlin, 1971. MR 0354651 Zbl 0234.14002

[Weyl 1997] H. Weyl, The classical groups: Their invariants and representations, Princeton University, 1997. MR 98k:01049 Zbl 1024.20501

Communicated by Ravi Vakil

Received 2010-07-23 Revised 2011-04-15 Accepted 2011-06-30

kwlan@math.umn.edu

Department of Mathematics, Princeton University, and School of Mathematics, Institute for Advanced Study, Princeton, NJ, United States

Current address:

School of Mathematics, University of Minnesota, Vincent Hall, 206 Church Street SE, Minneapolis, MN 55455, United States 


\section{Algebra \& Number Theory}

msp.berkeley.edu/ant

\section{EDITORS}

MANAGING EDITOR

Bjorn Poonen

Massachusetts Institute of Technology

Cambridge, USA

\author{
EDITORIAL BOARD CHAIR \\ David Eisenbud \\ University of California \\ Berkeley, USA
}

\section{BOARD OF EDITORS}

Georgia Benkart

Dave Benson

Richard E. Borcherds

John H. Coates

J-L. Colliot-Thélène

Brian D. Conrad

Hélène Esnault

Hubert Flenner

Edward Frenkel

Andrew Granville

Joseph Gubeladze

Ehud Hrushovski

Craig Huneke

Mikhail Kapranov

Yujiro Kawamata

János Kollár

Yuri Manin

Barry Mazur

Philippe Michel
University of Wisconsin, Madison, USA

University of Aberdeen, Scotland

University of California, Berkeley, USA

University of Cambridge, UK

CNRS, Université Paris-Sud, France

University of Michigan, USA

Universität Duisburg-Essen, Germany

Ruhr-Universität, Germany

University of California, Berkeley, USA

Université de Montréal, Canada

San Francisco State University, USA

Hebrew University, Israel

University of Kansas, USA

Yale University, USA

University of Tokyo, Japan

Princeton University, USA

Northwestern University, USA

Harvard University, USA

École Polytechnique Fédérale de Lausanne
Susan Montgomery

Shigefumi Mori

Raman Parimala

Jonathan Pila

Victor Reiner

Karl Rubin

Peter Sarnak

Joseph H. Silverman

Michael Singer

Vasudevan Srinivas

J. Toby Stafford

Bernd Sturmfels

Richard Taylor

Ravi Vakil

Michel van den Bergh

Marie-France Vignéras

Kei-Ichi Watanabe

Andrei Zelevinsky

Efim Zelmanov
University of Southern California, USA

RIMS, Kyoto University, Japan

Emory University, USA

University of Oxford, UK

University of Minnesota, USA

University of California, Irvine, USA

Princeton University, USA

Brown University, USA

North Carolina State University, USA

Tata Inst. of Fund. Research, India

University of Michigan, USA

University of California, Berkeley, USA

Harvard University, USA

Stanford University, USA

Hasselt University, Belgium

Université Paris VII, France

Nihon University, Japan

Northeastern University, USA

University of California, San Diego, USA

\section{PRODUCTION}

contact@msp.org

Silvio Levy, Scientific Editor

See inside back cover or www.jant.org for submission instructions.

The subscription price for 2012 is US \$175/year for the electronic version, and \$275/year (+ \$40 shipping outside the US) for print and electronic. Subscriptions, requests for back issues from the last three years and changes of subscribers address should be sent to Mathematical Sciences Publishers, Department of Mathematics, University of California, Berkeley, CA 94720-3840, USA.

Algebra \& Number Theory (ISSN 1937-0652) at Mathematical Sciences Publishers, Department of Mathematics, University of California, Berkeley, CA 94720-3840 is published continuously online. Periodical rate postage paid at Berkeley, CA 94704, and additional mailing offices.

ANT peer review and production are managed by EditFLOW ${ }^{\circledR}$ from Mathematical Sciences Publishers.

PUBLISHED BY

mathematical sciences publishers

http://msp.org/

A NON-PROFIT CORPORATION

Typeset in IATEX

Copyright $@ 2012$ by Mathematical Sciences Publishers 


\section{Algebra \& Number Theory}

Volume $6 \quad$ No. $5 \quad 2012$

Fields of moduli of three-point $G$-covers with cyclic $p$-Sylow, I

ANDREW OBUS

Toroidal compactifications of PEL-type Kuga families

KAI-WEN LAN

Idempotents in representation rings of quivers

RYAN KINSER and RALF SCHIFFLER

Cox rings and pseudoeffective cones of projectivized toric vector bundles

José González, Milena Hering, Sam Payne and Hendrik SÜss

Squareful numbers in hyperplanes

KARL VAN VALCKENBORGH

A denominator identity for affine Lie superalgebras with zero dual Coxeter number MARIA GORELIK and SHIFRA REIF 